\title{
Characterization of Lipoxygenases from Cyanothece sp.
}

\author{
Dissertation \\ For the award of the degree \\ “Doctor rerum naturalium” \\ of the Georg-August Universität Göttingen
}

Submitted by

Julia Newie

Born in

Wernigerode

Göttingen, January 2016 



\section{Members of the Examination Board}

Reviewer 1

Prof. Dr. Ivo Feußner

Department for Plant Biochemistry

Albrecht-von-Haller-Institute for Plant Sciences

University of Göttingen

Reviewer 2

Prof. Dr. Kai Tittmann

Department of Molecular Enzymology

Albrecht-von-Haller-Institute for Plant Sciences

University of Göttingen

Prof. Dr. Ralf Ficner

Department of Molecular Structural Biology

Institute for Microbiology and Genetics

University of Göttingen

Prof. Dr. Marina Bennati

Electron Paramagnetic Resonance Spectroscopy Group

Max Planck Institute for Biophysical Chemistry

Göttingen

Prof. Dr. Ricardo Mata

Computational Chemistry and Biochemistry Group

Institute of Physical Chemistry

University of Göttingen

Prof. Dr. Ulf Diederichsen

Department of Organic and Biomolecular Chemistry

University of Göttingen 


\section{Table of contents}

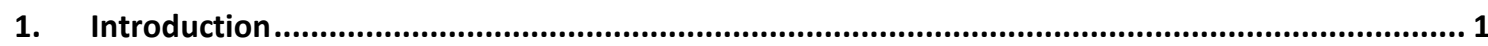

$1.1 \quad$ Lipids and oxilipins are vital biomolecules ..................................................... 1

1.2 Lipid autoxidation occurs in a non-enzymatic reaction.......................................... 1

1.3 Enzymatic lipid oxidation leads to a variety of signaling molecules........................... 2

1.4 The LOX reaction proceeds as a catalytic cycle ............................................. 3

1.4.1 LOX activation..................................................................................... 4

1.4.2 Hydrogen abstraction and radical rearrangement ......................................... 4

1.4.3 Oxygen insertion and peroxyl radical reduction ............................................. 4

1.5 The LOX structure is in general composed of two domains.................................... 5

1.5.1 The catalytic LOX domain comprises the non-heme iron ................................ 6

1.5.2 The $\mathrm{N}$-terminal $\beta$-barrel domain probably mediates membrane binding ............ 7

1.5.3 Substrate binding channels are boot-shaped to U-shaped ..............................8

1.5.4 Dioxygen transport to the active site may involve a separate oxygen channel....9

1.6 Control of oxygenation specificity by LOXs is based on three models...................... 9

1.6.1 Selection of a specific pentadiene is achieved by frame shift of the substrate.. 10

1.6.2 The substrate orientation determines the side of the pentadiene for LOX catalysis............................................................................................ 12

1.6.3 The Gly/Ala switch controls the oxygen access to one end of the pentadiene .. 12

1.7 Manganese LOXs produce bis-allylic hydroperoxides ........................................ 13

1.8 Bis-allylic hydroperoxides are also formed by an iron-containing LOX..................... 13

1.9 Theoretical concepts for specific oxygenation on one pentadiene ......................... 15

1.10 Cyanothece sp. harbors two LOX genes .......................................................... 17

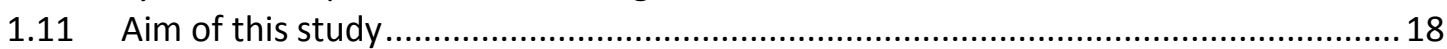

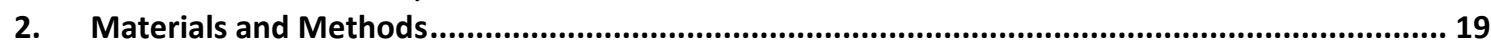

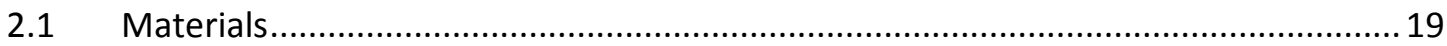

2.1.1 Chemicals........................................................................................ 19

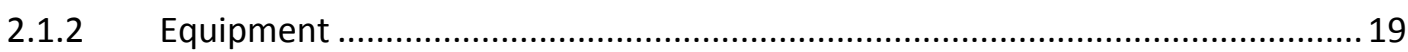

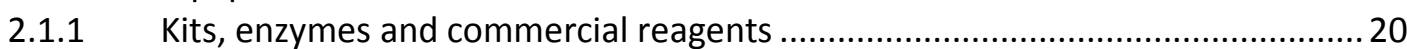

2.1.2 Data analysis and presentation ........................................................... 20

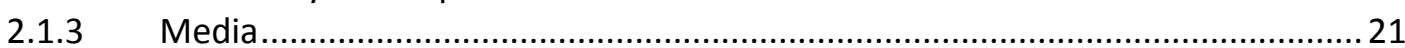

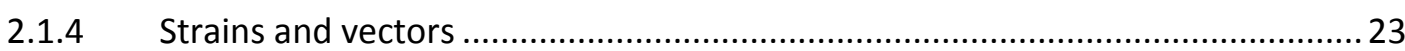

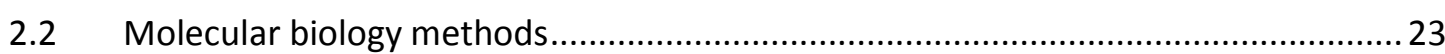

2.2.1 Plasmid DNA isolation ....................................................................... 23

2.2.2 Preparation of chemically competent E. coli cells and transformation of plasmid DNA.................................................................................. 23

2.2.3 Amplification of DNA fragments by polymerase chain reaction ....................... 24

2.2.4 DNA sequence analysis ...................................................................... 26

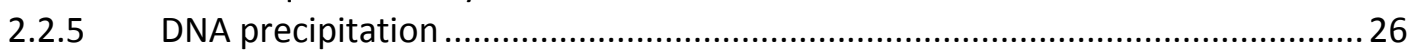

2.2.6 Ligation of compatible DNA fragments ................................................... 26

2.2.7 Agarose gel electrophoresis ............................................................ 27

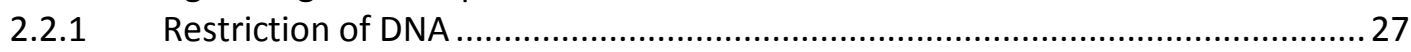

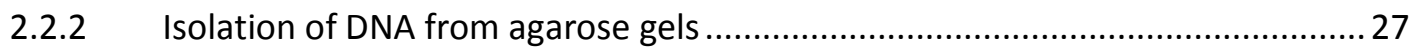

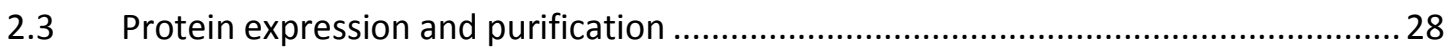

2.3.1 Cultivation of $E$. coli for protein expression ............................................... 28

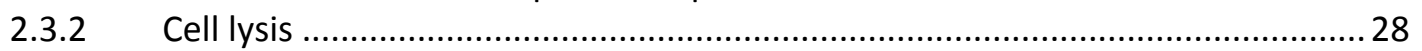

2.3.3 Protein purification by immobilized metal ion affinity chromatography........... 29

2.3.4 Size-exclusion chromatography............................................................... 29

2.3.5 Metal cofactor substitution by expression in minimal medium ....................... 30

$2.4 \quad$ Protein analysis and biochemical characterization .............................................. 30

2.4.1 Quantification of purified protein .......................................................... 30 
2.4.2 Protein analysis by polyacrylamide gel electrophoresis ................................... 31

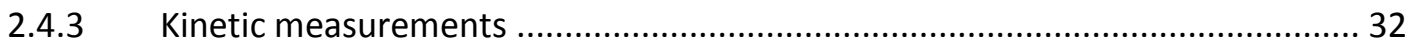

2.4.4 Analysis of the metal content by inductively coupled plasma atomic emission

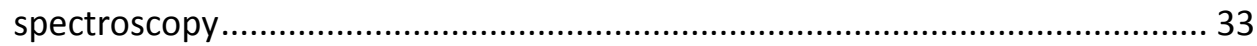

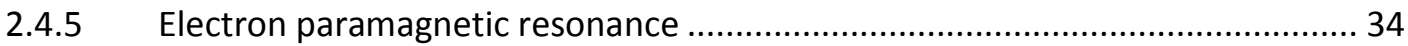

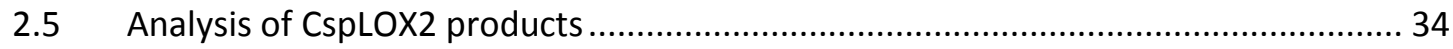

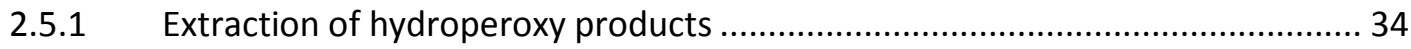

2.5.2 High-performance liquid chromatography analysis of lipoxygenation products 35

2.5.3 Fragmentation analysis by UHPLC-ESI-QTOF-MS/MS ..................................... 37

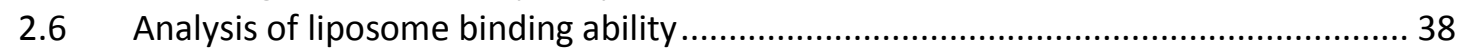

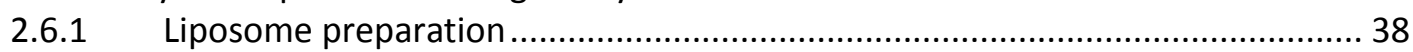

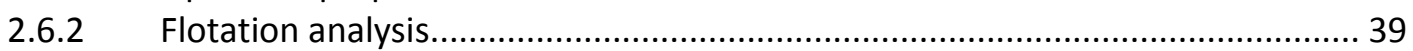

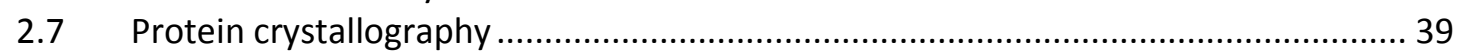

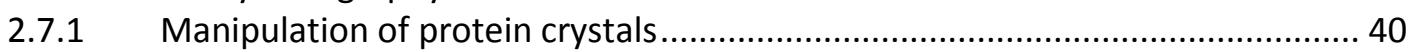

2.7.2 Data collection and structure determination .................................................. 41

2.8 Molecular dynamics and discrete Fourier transform simulations ............................. 41

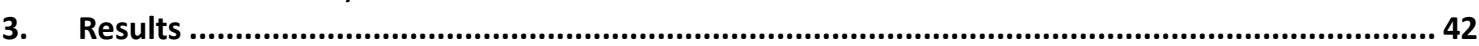

3.1 Cyanothece sp. expresses two LOXs with conserved catalytic domains but unusual

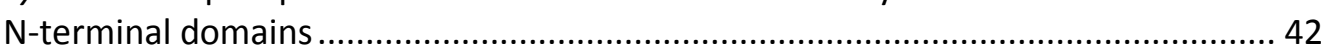

3.1.1 Sequence similarity of CspLOX1 to other LOXs is very low............................... 42

3.1.2 The catalytic domains of CspLOX1 and CspLOX2 resemble other LOXs ............. 44

3.1.3 The N-terminal domains of LOXs from Cyanothece sp. PCC8801 exhibit

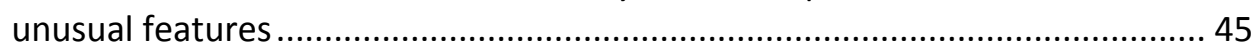

3.1.4 The $\alpha$-helical extension of CspLOX1 mediates binding to liposomes ................. 48

3.2 CspLOX2 produces the instable 11-HPODE as an intermediate ................................ 51

3.2.1 Optimized protocol required for reproducible 11-HPODE determination ......... 51

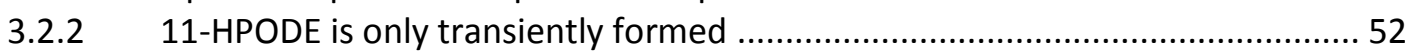

3.2.3 Activation of CspLOX2 with HPODEs removes the lag phase ........................... 54

3.2.4 Kinetics of the isomerization reaction exclude model of reaction sequence..... 55

3.2.5 11-HPODE is the major product of the first reaction phase .............................. 57

3.2.6 Some other LOXs can also isomerize 11-HPODE to conjugated HPODEs ............58

3.3 EPR and ICP-AES studies reveal that CspLOX2 is only active with iron .......................59 59

3.3.1 The iron cofactor of CspLOX2 can be substituted with manganese .................... 59

3.3.2 The coordination of manganese in the active site is identical to that of iron .... 61

3.4 The active site clamp controls the oxygenation specificity .......................................63 63

3.4.1 Residues in the core of the active site are important for the CspLOX2

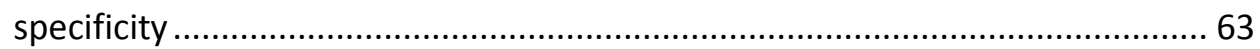

3.4.2 The residues of the active site clamp not only influence the positional specificity, but also the stereospecificity of the products.................................. 68

3.5 Attempts to obtain a structure of an enzyme substrate complex failed.................... 70

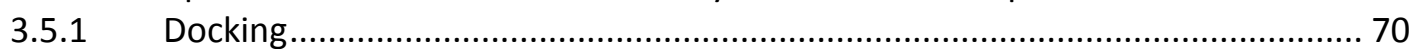

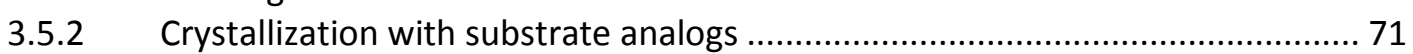

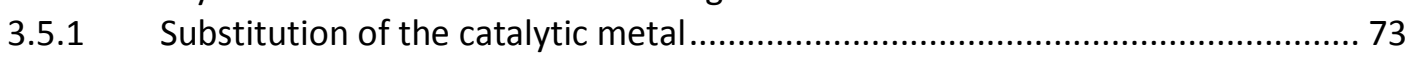

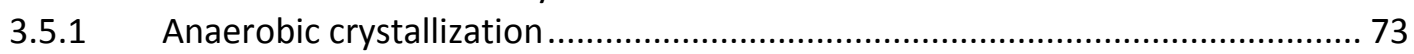

3.6 Mimicking the CspLOX2 active site induces 11-HPODE in another LOX .................... 75

3.6.1 Exchange of two amino acids in CspLOX1 sufficient for 11-HPODE formation .. 75

3.7 The putative oxygen channel of $8 R$-LOX is also present in CspLOX2 ........................ 80

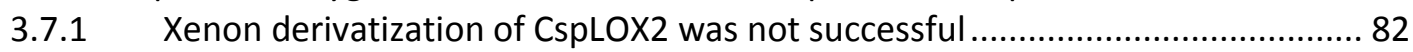

3.8 No suitable hydrogen atom donors are found in the CspLOX2 active site ................. 83

3.9 Computational approach supports role of steric shielding for 11-HPODE formation 85 
4. Discussion

4.1 The N-termimal helices of CspLOX1 may be involved in substrate acquisition from membranes

4.2 Although 11-HPODE is formed and isomerized in a very similar way in MnLOXs and CspLOX2, the catalytic metal is not interchangeable.

4.3 The most important factors for 11-HPODE formation in CspLOX2 may include

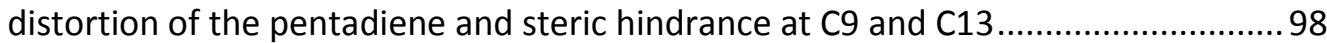

4.3.1 Radical localization ............................................................................ 100

4.3.2 Selective peroxyl radical trapping ........................................................ 101

4.3.3 Oxygen channel .................................................................................. 102

Steric shielding..................................................................................... 103

4.4 The concepts may also apply to MnLOXs and classical LOXs with only conjugated

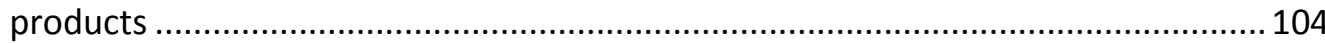

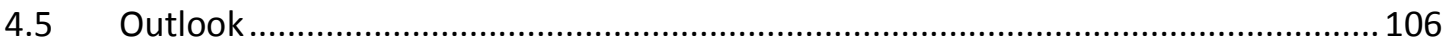

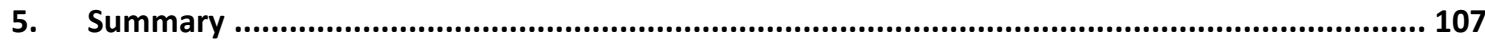

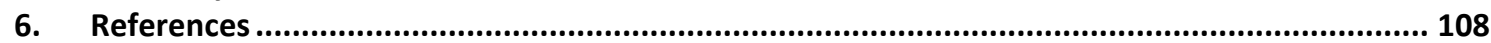

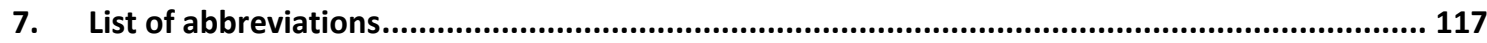

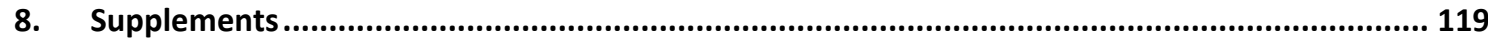




\section{Introduction}

\subsection{Lipids and oxilipins are vital biomolecules}

Lipids are important biomolecules that fulfill a variety of biological functions in living cells. They are for instance the building block of biological membranes to separate cell compartments from each other and are used for energy storage (Berg et al., 2004). In addition, lipids are vital signaling molecules in eukaryotes (Martin, 1998; Wang et al., 2006; Xue et al., 2007). One major class of lipid mediators are the so-called oxylipins which comprise oxidized fatty acids and their derivatives. In aerobic environments these oxidized fatty acids can be formed by specific enzymes or in a non-enzymatic process called autoxidation. Especially polyunsaturated fatty acids (PUFAs) are prone to oxidation. These PUFAs are in general abbreviated by indicating their number of carbon atoms $(X)$ and the number of double bonds $(Y)$ as well as the distance of the last double bond from the methyl end $(n-Z)$ in the form of $X: Y(n-Z)$. Common PUFAs in biological systems include linoleic acid (18:2(n-6)), $\alpha$-linolenic acid (18:3(n-3)) or arachidonic acid (20:4(n6)) (Figure 1).

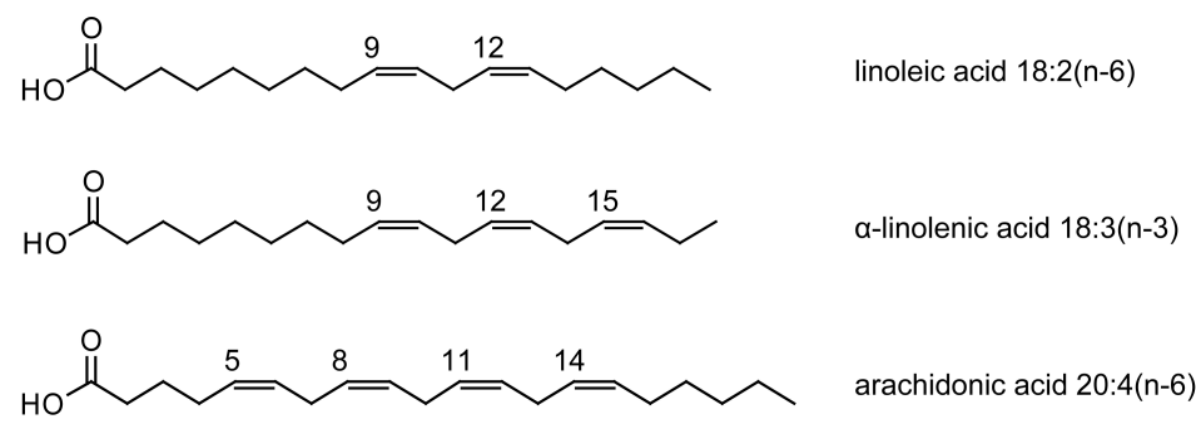

Figure 1. Common PUFAs in living cells.

\subsection{Lipid autoxidation occurs in a non-enzymatic reaction}

The process of lipid autoxidation is initiated by the formation of a carbon-centered lipid radical L. (Figure 2). The radical is propagated by addition of molecular oxygen and generation of a peroxyl radical (L-OO.) which in turn abstracts a hydrogen atom from another lipid molecule $(\mathrm{L}-\mathrm{H})$ to produce a new lipid radical ( $\mathrm{L} \cdot)$ and a fatty acid hydroperoxide $(\mathrm{L}-\mathrm{OOH})$. The result of this process is a chain reaction which can only be terminated by fusion with another radical or by the action of an antioxidant (ArO-H) (Yin and Porter, 2005).

Since radicals are highly reactive compounds, it is conceivable that lipid peroxidation is not only related to the formation of important lipid mediators, but can have deleterious effects on living cells. In mammals, lipid oxidation may lead to numerous diseases, such as cancer, diabetes, arteriosclerosis, chronic rheumatoid arthritis, inflammatory bowel disease and neurodegenerative disorders such as Alzheimer disease and Parkinson's disease (Porter et al., 1995). The benefits of antioxidants in the diet has largely been advertised. These antioxidants 
are assumed to play a role in counteracting lipid oxidation by trapping fatty acid peroxyl radicals (Yin and Porter, 2005) (Figure 2, Step 4).

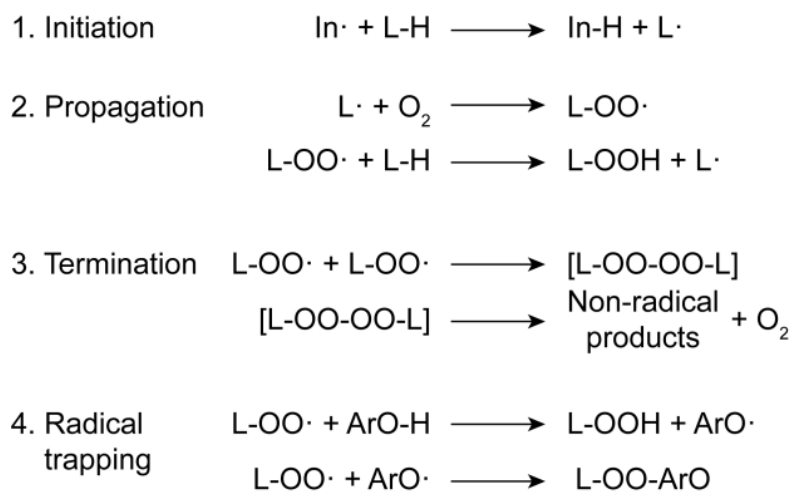

Figure 2. Process of lipid autoxidation. After formation of a lipid radical (L·) by an initiator (In·), oxygen is added and a peroxyl radical (L-OO·) is formed. This radical can react with new lipid molecules to produce new carbon centered radicals. The chain reaction is terminated by fusion of two radicals or by radical trapping with an antioxidant $(\mathrm{ArO}-\mathrm{H})$ (Yin and Porter, 2005).

\subsection{Enzymatic lipid oxidation leads to a variety of signaling molecules}

The process of lipid oxidation can also occur in a regulated biological reaction catalyzed by enzymes. The initial step of lipid peroxidation is either catalyzed by cyclooxygenases (COXs), $\alpha$ dioxygenases ( $\alpha$-DOXs) or by lipoxygenases (LOXs). All of these enzymes generate a lipid radical (L.) which subsequently allows the addition of dioxygen. After initial peroxidation of the lipid substrate, cascades of different enzymatic reactions can follow and lead to a variety of oxidized fatty acid derivatives. These oxylipins have important functions in intra- and intermolecular signaling in animals, plants and fungi (Andreou et al., 2009; Kühn et al., 2015). In mammals for example, prostaglandins and leukotrienes which belong to the group of eicosanoids present the most important group of oxylipins. These lipid mediators are produced to regulate immune responses such as inflammation and fever (Funk, 2001). In plants, oxylipins include fatty acid hydroperoxides and hydroxides as well as oxo-, keto- and epoxy fatty acid derivatives. The most important hormone produced in the oxylipin pathway of plants is jasmonic acid including its derivatives. This plant hormone is involved in defense to pathogens, development of reproductive organs and plant growth (Andreou et al., 2009). The biological roles of oxylipins have also been studied in fungi. Here, they function for example in the regulation of the life cycle and mycotoxin production (Noverr et al., 2002; Tsitsigiannis and Keller, 2007). Although LOX genes were also found in some mosses, algae and bacteria, suggesting that oxidized lipids are also enzymatically produced in these organisms, their functions remain largely elusive (Horn et al., 2015).

The focus of this study will be on LOXs and their oxygenation specificity. Similar to autoxidation, they insert molecular oxygen into PUFAs with one or more cis,cis-1,4-pentadiene system. This reaction is, however, catalyzed in a highly regio- and stereospecific way to yield a single fatty acid hydroperoxide product (Figure 3). 


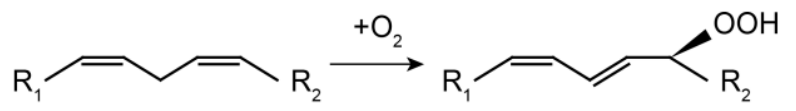

Figure 3. LOX reaction. LOXs catalyze the insertion of molecular oxygen into PUFAs to yield a specific fatty acid hydroperoxide.

It should be noted that different LOX specificities result in distinct oxylipins with different biological functions. For instance, only the 13S-hydroperoxide of $\alpha$-linolenic acid is a precursor for jasmonic acid synthesis, while other isomers are not used as substrate for this pathway (Andreou et al., 2009). The effect is even more evident in mammals, where different LOX specificities may even have opposite functions. While the activity of 5-LOX leads to the formation of proinflammatory leukotrienes, a combination of 15- and 5-LOX or 12- and 5-LOX activity results in the synthesis of anti-inflammatory lipoxines (Kühn and O'Donnell, 2006; Steinhilber, 1999). It is therefore conceivable that numerous studies have focused on the enzymology of these important enzymes.

\subsection{The LOX reaction proceeds as a catalytic cycle}

The LOX reaction always proceeds at one of the pentadiene systems of the substrate. During the LOX reaction cycle, the catalytic non-heme iron in the active site alternates between the ferric $\mathrm{Fe}$ (III) and ferrous Fe(II) state. The cycle includes four steps: (1) hydrogen abstraction from the middle position of the pentadiene, (2) radical rearrangement within the pentadiene system, (3) dioxygen insertion at either end of the five carbon moiety and (4) final reduction of the peroxyl radical to yield a hydroperoxide (Ivanov et al., 2010) (Figure 4).
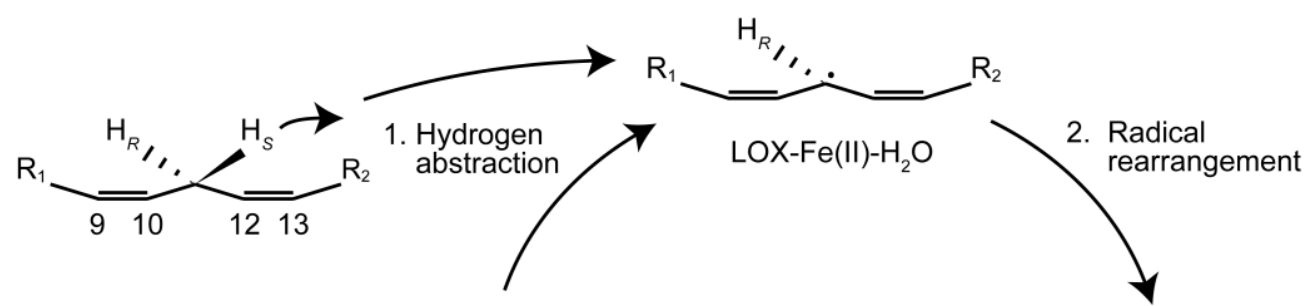

LOX-Fe(III)-OH
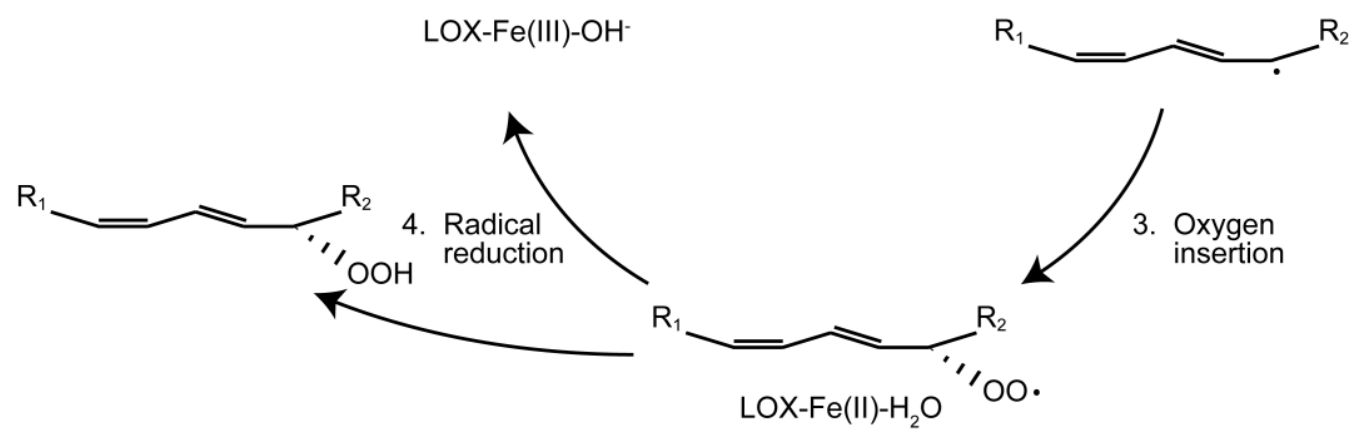

Figure 4. The reaction cycle of the LOX reaction. LOX catalysis is assumed to include four steps: hydrogen abstraction, radical rearrangement, oxygen insertion and radical reduction (Ivanov et al., 2010). The numbering of the substrate in this model corresponds to linoleic acid used as example. 


\subsubsection{LOX activation}

In the as-isolated state of LOXs, Fe(II) is in general found in the active site. But due to the fact that $\mathrm{Fe}(\mathrm{III})$ is required for the active state, the enzyme has to be activated first. This activation process is catalyzed by reaction products, i.e. fatty acid hydroperoxides, which oxidize the inactive $\mathrm{Fe}$ (II) to the active $\mathrm{Fe}$ (III) cofactor. Thereafter, $\mathrm{Fe}$ (III) can catalyze the hydrogen abstraction and initiate the catalytic cycle. This activation phase is related to a lag phase usually observed at the beginning of the LOX reaction (Aharony and Stein, 1986; Jones et al., 1996; Smith and Lands, 1972).

\subsubsection{Hydrogen abstraction and radical rearrangement}

Once the catalytic iron is in its active Fe(III) state, it will abstract a hydrogen atom from the bisallylic methylene group of the cis,cis-1,4-pentadiene system (Figure 4, Step 1). This subsequently leads to the formation of a carbon-centered radical. Experiments with deuterated substrates suggested that the pro-S hydrogen is abstracted in most LOXs (Moiseyev et al., 1997; Rickert and Klinman, 1999). It should be noted that this step, which is accompanied by a large kinetic isotope effect, is the rate limiting step of this reaction. In fact, the LOX reaction exhibits one of the largest isotope effects ever measured. Since the kinetic isotope effect of the pro-S deuterium is larger than what can be explained by the classical theory, the effects were interpreted by quantum mechanics and hydrogen tunneling was proposed to be the primary mechanism of hydrogen transfer (Lewis et al., 1999; Moiseyev et al., 1997; Rickert and Klinman, 1999). During this process, hydrogen tunnels first from the substrate to the enzyme and subsequently the electron of the hydrogen is transferred to the catalytic iron, thereby reducing it from $\mathrm{Fe}$ (III) to $\mathrm{Fe}$ (II). The sixth ligand of the iron, which is assumed to be a hydroxide (LOX-Fe(III)-OH'), may receive the hydrogen from the substrate to form water (LOX-Fe(II)- $\mathrm{H}_{2} \mathrm{O}$ ) (Tomchick et al., 2001). The carboncentered radical formed on the middle position of the pentadiene system will rapidly delocalize over the pentadiene moiety, due to the conjugation with the double bonds. It will thus be found at $\mathrm{C} 1, \mathrm{C} 3$ or $\mathrm{C} 5$ of the five carbon structure (Figure 4, Step 2).

\subsubsection{Oxygen insertion and peroxyl radical reduction}

The radical itself is subsequently reactive enough to drive the addition of dioxygen (Figure 4, Step 3). A peroxyl radical is formed, similar to the propagation step of the autoxidation (Chapter 1.2). In general, oxygen is inserted at the $n+2$ or $n-2$ position relative to the hydrogen abstraction in an antarafacial way (opposite of the hydrogen abstraction) (Figure 5). Although both products are formed by addition of oxygen to the same side of the substrate, the $R$-enantiomer is produced in the $\mathrm{n}-2$ position, whereas the $S$-enantiomer is formed at the $\mathrm{n}+2$ position according to the Cahn-Ingold-Prelog priority rules (Figure 5). During dioxygen insertion at the $n+2$ or $n-2$ position, conjugated double bonds are formed. 


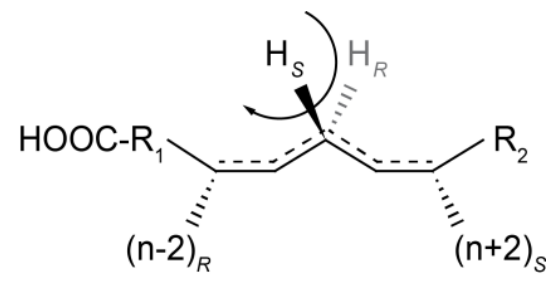

Figure 5. Antarafacial relationship of hydrogen abstraction and dioxygen insertion. Hydrogen abstraction and dioxygen insertion occur from different sides of the substrate molecule.

The LOX reaction is assumed to be terminated by proton coupled electron transfer to the peroxyl radical (Figure 4, Step 4). The reaction cycle would be complete by transfer of the initially abstracted hydrogen from $\mathrm{Fe}(\mathrm{II})-\mathrm{H}_{2} \mathrm{O}$ to the peroxyl radical, thereby recovering the catalytically active form $\mathrm{Fe}(\mathrm{III})-\mathrm{OH}^{-}$that can abstract a new hydrogen atom from another PUFA molecule (Tomchick et al., 2001).

\subsection{The LOX structure is in general composed of two domains}

The first LOX structure being solved was the one of the soybean LOX1 in 1993 (Boyington et al., 1993) which was later refined to $1.4 \AA$ (Minor et al., 1996). Since then, crystal structures of 12 different LOX enzymes have been deposited in the Protein Data Bank (PDB). They comprise four plant and seven animal enzymes (Supplemental Tables 1-4). The only bacterial structure has recently been solved for the Pseudomonas aeruginosa LOX (Garreta et al., 2013).

LOX enzymes share a common fold and belong to the same gene family, even though the sequence homology with only $25-40 \%$ identity is rather low. Plant LOXs have molecular weights of 94-104 kDa (Brash, 1999) and are thus remarkably larger than animal LOXs which show molecular weights of 75-80 kDa (Funk, 1996). This difference in size can be explained by some plant LOX specific loop regions missing in animal LOX enzymes (Newcomer and Brash, 2015). LOXs generally consist of two domains: a larger C-terminal catalytic domain and a smaller $\mathrm{N}$ terminal $\beta$-barrel domain (Figure 6A). While the catalytic LOX domain is present in all LOXs, some bacterial LOXs exhibit a reduction of the $\mathrm{N}$-terminal domain. The crystal structure of the $P$. aeruginosa LOX for example revealed a lack of the $\beta$-barrel domain. Instead, an $\alpha$-helical insertion covers the protein surface that is otherwise shielded by the $\beta$-barrel domain (Garreta et al., 2013) (Figure 6B). Other fully functional bacterial LOXs from Cyanothece sp. (Andreou et al., 2010), Nostoc puntiforme (Koeduka et al., 2007) and Burkholderia thailandensis (An et al., 2015) were also characterized as so-called mini-LOX lacking the $\beta$-barrel domain. Moreover, some LOXs from corals or bacteria were described as fusion proteins that carry an $\mathrm{N}$-terminal domain with an additional enzymatic activity (Boutaud and Brash, 1999; Gao et al., 2010; Lang et al., 2008; Lõhelaid et al., 2008; Zhang et al., 2012; Zheng et al., 2008). 

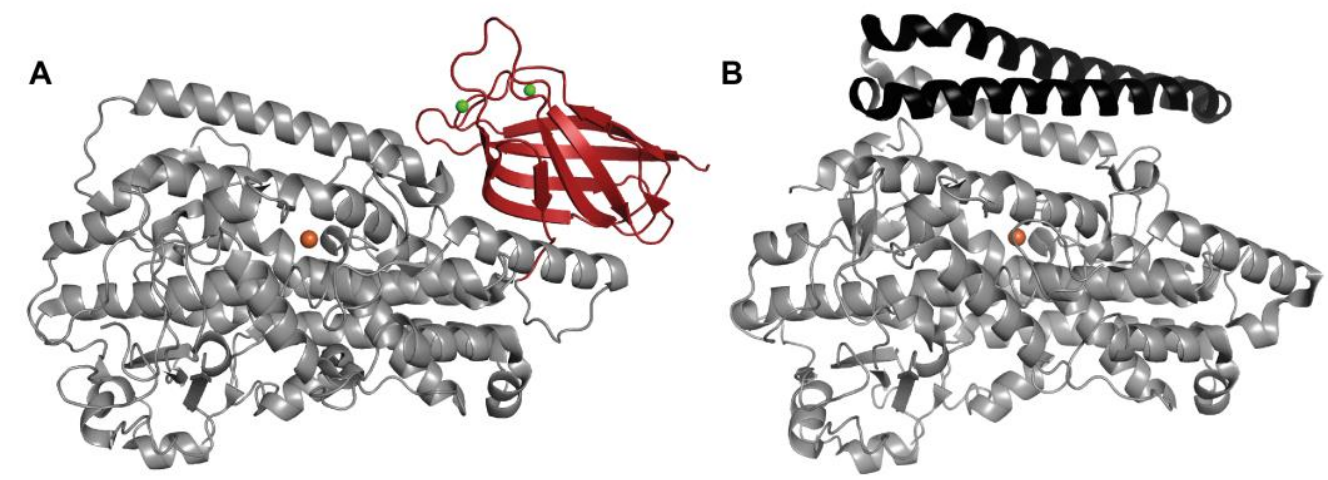

Figure 6. LOX structure. (A) Typical two-domain structure of coral 8R-LOX. The C-terminal catalytic domain consisting of mainly $\alpha$-helices is depicted in grey and the $\mathrm{N}$-terminal $\beta$-barrel domain in red. The non-heme iron cofactor (orange sphere) is buried in the core of the catalytic domain. $\mathrm{Ca}^{2+}$ (green) is bound to the $\beta$-barrel domain. (B) Instead of the $\beta$-barrel domain, a long helical insertion (black) is found in the $P$. aeruginosa 15-LOX structure.

\subsubsection{The catalytic LOX domain comprises the non-heme iron}

The large catalytic domain is structurally conserved in all LOXs. It consists of $\sim 17 \alpha$-helices and comprises the active site (Newcomer and Brash, 2015). In the active site of the catalytic domain, the 2-His-1-carboxylate facial triade is found as a common structural motif. This motif is characteristic for mononuclear non-heme iron enzymes as it is involved in the coordination of the metal. Five amino acids including three histidines, an asparagine and the carboxyl group of a C-terminal isoleucine are involved in the pseudo octahedral coordination of the catalytic iron (Figure 7). In some LOX structures the sixth position is occupied by a water or hydroxide (Minor et al., 1996; Segraves et al., 2006; Xu et al., 2012). Only in few mammalian LOXs a forth histidine or a serine is found at the position of the asparagine (Supplemental Figure 4).
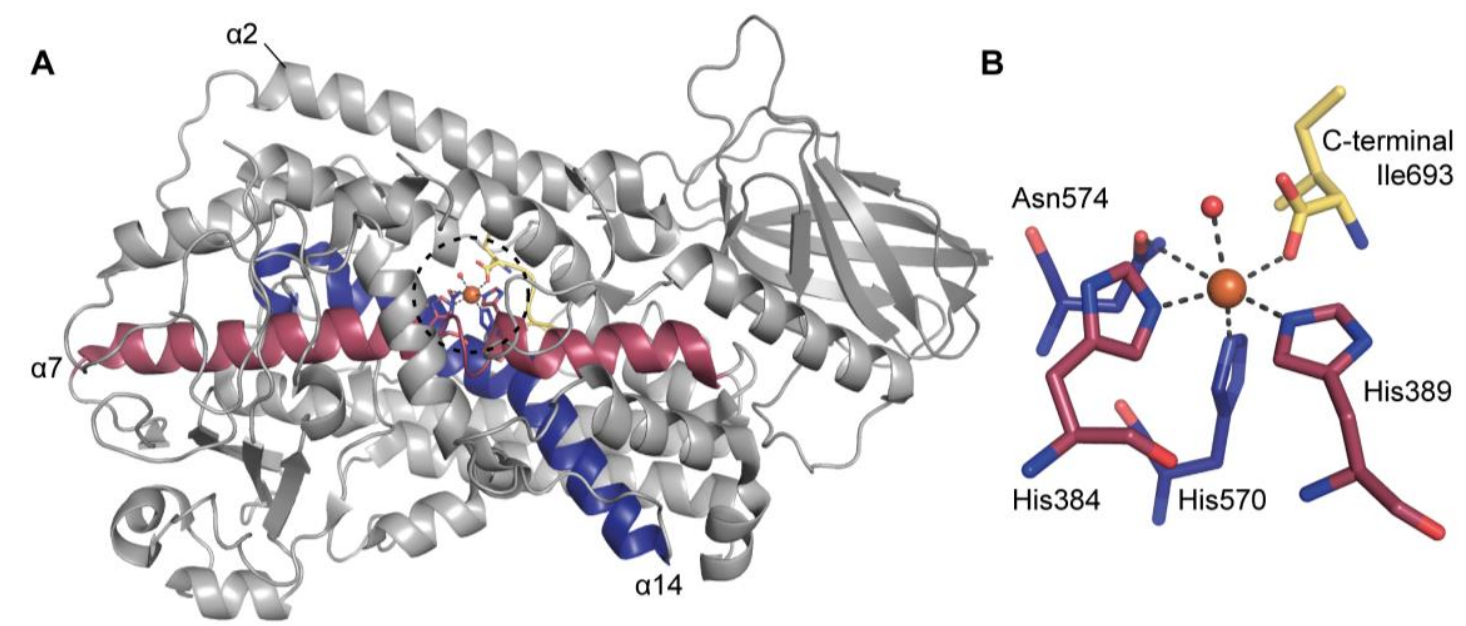

Figure 7. Coordination of the metal cofactor. The coordination sphere is exemplified on $8 R$-LOX. (A) The two long helices $\alpha 7$ (purple) and $\alpha 14$ (blue) contribute the histidine and asparagine residues of the coordination sphere. (B) Iron (orange sphere) is pseudo octahedrally coordinated by three histidines, an asparagine and the carboxyl group of the C-terminal isoleucine. The sixth position is assumed to be occupied by a water or hydroxide molecule (small red sphere). 
The conserved histidine and asparagine residues of the coordination sphere are contributed by two long helices ( $\alpha 7$ and $\alpha 14$ ) of the catalytic domain (Figure 7A). The carboxyl group of the isoleucine at the C-terminus of the peptide chain inserts into the active site to position itself properly to the iron (Figure 7B, yellow). Predominantly main-chain to main-chain hydrogen bonds are involved in holding the C-terminus in place. In addition, an asparagine residue located four amino acids upstream of the C-terminus forms main-chain to main-chain, main-chain to side-chain, and side-chain to main-chain hydrogen bonds which explains its high degree of conservation. In comparison, the hydrophobic interactions of the isoleucine side chain itself are limited and it remains unknown why this residue is almost invariant (Newcomer and Brash, 2015).

\subsubsection{The $\mathrm{N}$-terminal $\beta$-barrel domain probably mediates membrane binding}

Although the fold of the N-terminal domain with about 25-30 kDa rather presents a $\beta$-sandwich, it is generally referred to as $\beta$-barrel domain or PLAT (Polycystin-1, Lipoxygenase, Alpha-Toxin) domain (Chen and Funk, 2001; Hammel et al., 2004; May et al., 2000; Newcomer and Brash, 2015). This domain exhibits a high similarity to the C2 module of many kinases and phospholipases (Corbin et al., 2007) which functions as $\mathrm{Ca}^{2+}$-dependent membrane-targeting domain in these enzymes. Due to this similarity, a membrane binding function was also proposed for the $\beta$-barrel domain of LOXs. Studies on cucumber 13-LOX (May et al., 2000), soybean LOX1 (Tatulian et al., 1998) and human LOXs (Kulkarni et al., 2002; Walther et al., 2011) supported this idea. Several peripheral tryptophans located at the outer loops of this domain were identified to be involved in the membrane interaction in human 5-LOX (Kulkarni et al., 2002). Furthermore, the crystal structures of coral $8 R$-LOX (Oldham et al., 2005), 11R-LOX (Eek et al., 2012) and 15-LOX2 (Kobe et al., 2014) revealed the $\mathrm{Ca}^{2+}$ binding sites with in the $\beta$-barrel domain (Figure $6 \mathrm{~A}$, green spheres). Binding of $\mathrm{Ca}^{2+}$ probably stabilizes loop regions within the $\beta$ barrel domain that may insert into the membrane. Interestingly, the $\mathrm{Ca}^{2+}$-coordinating amino acids exhibit only a low degree of conservation and also the putative membrane insertion loops differ between LOXs from different organisms (Newcomer and Brash, 2015). Although it was shown that this domain mediates membrane binding in 5-LOX and that truncations of this domain result in a reduced stability and catalytic activity (Ivanov et al., 2011; Walther et al., 2011), it is still a matter of debate whether the $\beta$-barrel domain has a role in substrate acquisition directly from membranes (Hammarberg et al., 2000; Ivanov et al., 2011; Kulkarni et al., 2002; Walther et al., 2011). Despite the fact that the $P$. aeruginosa 15-LOX lacks the $\beta$-barrel domain, it is nevertheless able to bind to membranes, suggesting that also other parts of the enzyme might mediate membrane binding (Garreta et al., 2013). 


\subsubsection{Substrate binding channels of LOXs are boot-shaped to U-shaped}

Even though soybean LOX1 was the first enzyme to be crystallized, it did not reveal a welldefined substrate binding channel to the catalytic iron (Minor et al., 1996). The structure of the 15-LOX in complex with an inhibitor was subsequently solved in 1998 and led to the model of a boot-shaped channel (Gillmor et al., 1998), which was also found in the soybean LOX3 structure with the hydroperoxide product (Skrzypczak-Jankun et al., 2001).

During the last three years, the model of how LOX substrates bind to the active site channel has largely been extended due to four crystal structures: the $P$. aeruginosa LOX in complex with a phospholipid (Garreta et al., 2013), the 12-LOX catalytic domain with an inhibitor (Xu et al., 2012), the human 15-LOX2 with a substrate-like detergent (Kobe et al., 2014) and especially the $8 R$-LOX with arachidonic acid obtained under anaerobic conditions (Neau et al., 2014). All of these structures reveal a U-shaped channel that is open at one end, which most likely represents the entrance to the channel, and closed at the other end, where the bottom of the pocket is formed. The catalytic iron is positioned at the base of the U-shaped channel (Figure 8). The channel is surrounded by a four-helix bundle (Newcomer and Brash, 2015). The bundle is composed of the two long helices that contribute the iron-coordinating residues to the active site and to the other side of the penultimate helix and helix $\alpha 8$ (Figure $8 \mathrm{~A}$ ). Of these, helix $\alpha 8$ exhibits an unusual curvature and is therefore referred to as "arched helix" (Gilbert et al., 2011; Ivanov et al., 2010; Neau et al., 2009). In addition to the iron-coordinating residues, a leucine and an isoleucine residue were found to be invariant in the LOX active site (Leu431 and Ile437 in the coral $8 R$-LOX) and were proposed to position the pentadiene for catalysis (Neau et al., 2014) (Figure 8B). The side chain of Arg182 located at the entrance to the channel is interacting with the fatty acid carboxylate in the $8 R$-LOX structure (Figure $8 \mathrm{~B}$ ). Although it plays an important role in positioning of the substrate and the Arg182Ala exchange causes a strong substrate inhibition (Neau et al., 2014), this residue is not conserved in other LOX orthologs (Newcomer and Brash, 2015). 

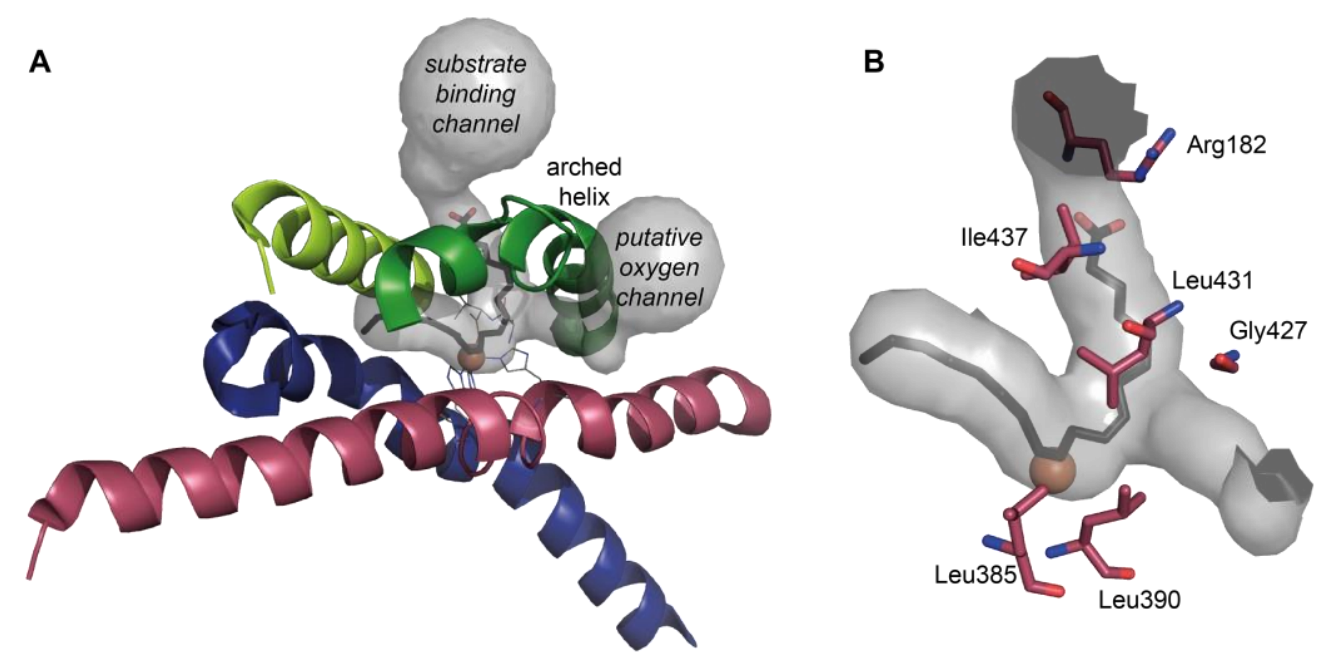

Figure 8. Substrate binding channel and putative oxygen channel in $8 R$-LOX. (A) Four helices of the catalytic core surround the active site: helix $\alpha 7$ (red), $\alpha 14$ (blue), the penultimate helix (light green) and the so-called arched helix (dark green). The arched helix separates the substrate channel from the putative oxygen channel. (B) Position of important amino acid residues in the $8 R$-LOX active site. Their role for catalysis is explained in the text.

\subsubsection{Dioxygen transport to the active site may involve a separate oxygen channel}

Since molecular oxygen is the second substrate of LOX enzymes, structural studies have also focused on putative oxygen transport pathways to the active site. A putative oxygen channel was first described for the soybean LOX1 (Knapp et al., 2001; Knapp and Klinman, 2003; Minor et al., 1996). Furthermore, a leucine residue was identified for regulating the oxygen transport to the active site in 15-LOX using a computational approach (Saam et al., 2007). This residue (Leu390 in $8 R$-LOX) is invariant and was proposed to help define the oxygen channel together with Leu385 of 8R-LOX (Figure 8B). In plants, a Trp-Ala pair was found at these two positions, which may also play a role in defining the oxygen channel (Newcomer and Brash, 2015).

Although the putative oxygen channel found in 8R-LOX (Figure 8) is also present in other LOXs, it not always reaches the surface of the protein (Newcomer and Brash, 2015). Direct experimental evidence for the existence of such an oxygen channel is still missing. However, the positional relationship of the iron cofactor and oxygen channel which are located on opposite sides of the substrate would nicely explain the antarafacial character of the LOX reaction (Neau et al., 2014).

\subsection{Control of oxygenation specificity by LOXs is based on three models}

As pointed out before, the LOX reaction initiates, controls and terminates the free radical reaction also observed for autoxidation (Chapter 1.2 and 1.4). But while autoxidation results in a mixture of different products, a single product with high regio- and stereospecificity is formed by LOXs. There are LOX enzymes known that catalyze the specific insertion of molecular oxygen at three of the four possible positions on linoleic acid $(9 R, 9 S, 13 S)$ (Andreou et al., 2009) and on 
10 of the 12 possible positions on arachidonic acid $(5 R, 5 S, 8 R, 8 S, 9 R, 11 R, 11 S, 12 R, 12 S$ and 15S) (Hada et al., 1997; Ivanov et al., 2010; Mortimer et al., 2006) (Figure 9).

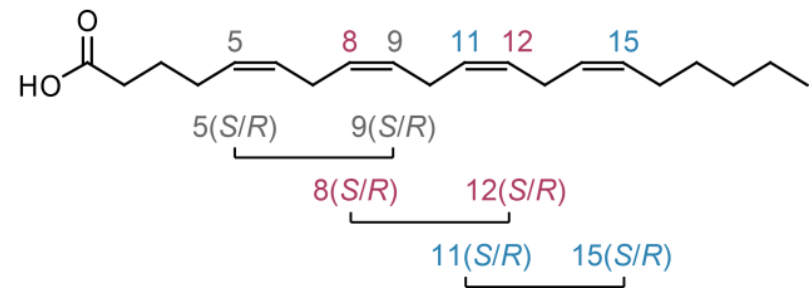

Figure 9. Products of arachidonic acid oxidation. Arachidonic acid contains three pentadiene systems. On each pentadiene system, four different products can be formed by autoxidation (grouped by colors). LOXs which are specific for each of the theoretical positions have been identified, except for $12 S$ and $15 R$ (Newcomer and Brash, 2015).

The specificity for a single chiral product is also reflected in the nomenclature of the enzymes. Mammalian 8R-LOXs for example insert molecular oxygen at position 8 of arachidonic acid in an $R$-conformation, while plant 13S-LOXs insert oxygen at position 13 of linoleic acid in an Sconformation. Based on structural data and mutational studies, different concepts were proposed that explain the LOX specificity. They include (A) the alignment of one selected pentadiene system to the catalytic iron, (B) selection of the side of the fatty acid for reaction by altering the orientation of the substrate and (C) accessibility of oxygen to the ends of the pentadiene system (Figure 10).

\subsubsection{Selection of a specific pentadiene is achieved by frame shift of the substrate}

In substrates containing more than one pentadiene system, like $\alpha$-linolenic acid $(18: 3(n-3))$ or arachidonic acid (20:4(n-3)) (Figure 1), hydrogen can be abstracted from more than one pentadiene system. The selection of a specific hydrogen atom for abstraction is assumed to be a result of the depth of the substrate binding channel. Depending on how deep the substrate can slide into the channel, a specific pentadiene will be exposed to the iron for attack (Kühn et al., 1990). This "frame-shift" of the fatty acid substrate can for example explain the difference of $12 S$ and 15S-lipoxygenation on arachidonic acid (Sloane et al., 1991) (Figure 10A). The model is strongly supported by experiments with artificial fatty acids that contain frame-shifted double bonds (Kühn et al., 1990). Furthermore, exchanging conserved differences between arachidonic acid 15S-LOX (Ile-Met) and 12S-LOX (Val-Ala) also exchanged the specificity (Sloane et al., 1991). It was later confirmed that these critical amino acids are located at the bottom of the substrate binding pocket, thereby defining the depth of the channel (Kühn, 2000). 
A
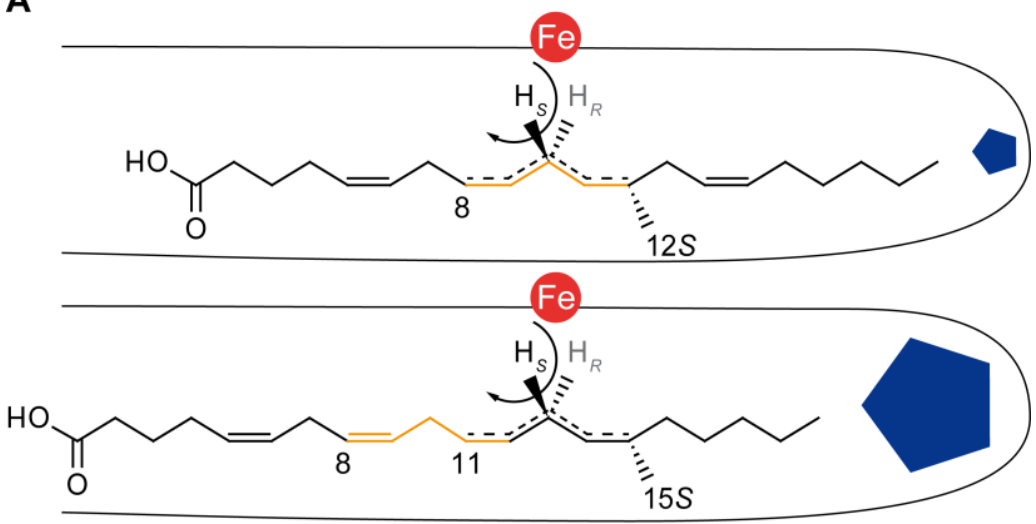

B
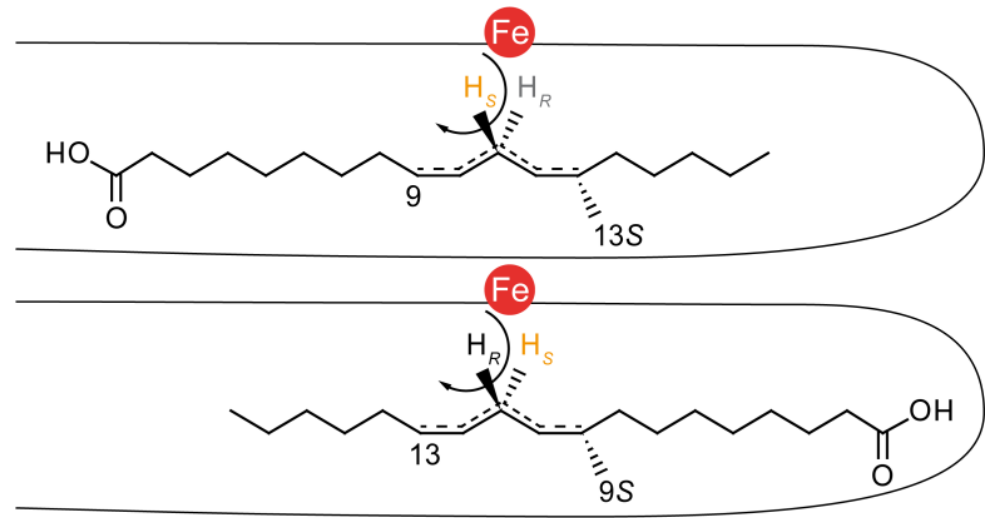

C
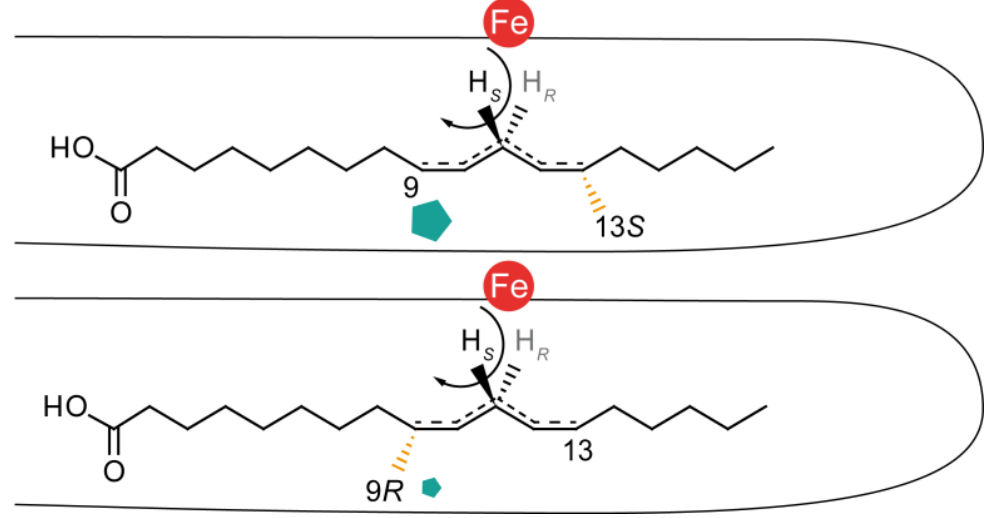

Figure 10. Conceptual basis of LOX specificity. (A) Frame-shift of the fatty acid substrate. Depending on the depth of the substrate binding channel a certain pentadiene system is exposed to the catalytic iron for hydrogen abstraction. The depth is influenced by the size of two residues (blue) at the bottom of the pocket (Sloane et al., 1991). (B) Orientation of the substrate. If the substrate enters the channel methyl end first (tail-first), the pro- $S$ hydrogen is abstracted, whereas the pro- $R$ hydrogen is abstracted if the carboxyl group of the substrate enters first (Egmond et al., 1972). (C) Determination of the oxygen insertion at one end of the pentadiene. A glycine at the conserved position (green) in the active site is associated with $R$-specific LOXs, while an alanine is assumed to shield this position and is thus associated with S-specific LOXs. This position functions as a "switch" to direct oxygen to one or the other end on the pentadiene system, thereby switching the $R / S$ specificity (Coffa and Brash, 2004). 


\subsubsection{The substrate orientation determines the stereospecificity of the hydrogen} abstraction

While substrate binding with the nonpolar methyl end first ("tail-first") has always been well accepted, the reversed orientation in which the carboxyl end enters the channel first ("headfirst") appeared more challenging due to the charge of the head group that would need to move through the long hydrophobic channel (Browner et al., 1998; Coffa et al., 2005a). Such a reversed substrate orientation was first concluded from the finding that 9S-LOXs and 13S-LOXs abstract hydrogen from different faces of the substrate: while 13S-LOXs abstract the pro-S hydrogen, 9SLOXs abstract the pro- $R$ hydrogen from $C 11$ of linoleic acid (Egmond et al., 1972). Since the positions for $9 S$ and 135 -hydroperoxides are also on different faces of the substrate, the antarafacial rule still holds true (Figure 10B). In fact, the positional relation between the hydrogen abstraction on the one side and the oxygen insertion at a deeper point in the channel on the other side is equivalent in both cases (Figure 10B). While this model was initially established for plant LOXs, the reversed binding of the substrate is also the only logical explanation for animal $5 S, 8 S$ and $12 R$ oxygenation specificities (Coffa et al., 2005a). A positively charged amino acid residue at the bottom of the substrate pocket in plant LOXs was discussed to be involved in the stabilization of the carboxyl group at the bottom of the substrate binding channel (Hornung et al., 1999). However, it was argued that free fatty acids which usually have a p $K_{\mathrm{a}}$ of pH 7-8 (Glickman and Klinman, 1995) would be uncharged at the optimal pH ( pH 6) of many LOXs with proposed carboxyl-end first binding (Butovich et al., 1998; Coffa et al., 2005b; Schneider et al., 2001).

\subsubsection{The Gly/Ala switch controls the oxygen access to one end of the pentadiene}

The two previously described concepts focus on the proper positioning of the pentadiene towards the iron. The positional relation of hydrogen abstraction and oxygen insertion remains unchanged in these models (Figure 10A and B). The concept developed by Coffa and colleagues (Coffa and Brash, 2004) directly addresses the question how oxygen is specifically inserted at one end or the other of the reacting pentadiene. Whether oxygen is inserted at the $n+2$ or the $\mathrm{n}-2$ position relative to the hydrogen abstraction also determines the stereospecificity of the product (Figure 5). Thus, abstraction of the same stereospecific pro-S hydrogen of linoleic acid leads to $9 R$ - and $13 S$-specific oxygen insertion, while abstraction of the pro- $R$ hydrogen in a reversed orientation results in $9 S$ - or $13 R$-specific products. A conserved residue in the active site which is occupied by an alanine in S-specific LOXs and a glycine in $R$-specific LOXs was identified. Exchanging this residue for the respective counterpart also switched the $R / S$ specificity by insertion of oxygen at the other end of the pentadiene system (Coffa and Brash, 2004) (Figure 10). Structural analysis later revealed that this position might sterically shield one end of the pentadiene system in case of an alanine, thus directing dioxygen to the other end (Knapp et al., 2001; Knapp and Klinman, 2003; Saam et al., 2007; Schneider et al., 2007). 


\subsection{Manganese LOXs produce bis-allylic hydroperoxides}

In addition to iron-containing LOXs, some fungal enzymes were characterized as manganesecontaining LOXs (MnLOXs). These MnLOXs include the 13R-MnLOX from Gaeumannomyces graminis (Su and Oliw, 1998), 9S-MnLOX from Magnaporte salvinii (Wennman and Oliw, 2013), Fo-MnLOX from Fusarium oxysporum and Cg-MnLOX from Colletotrichum gleosporioides (Wennman et al., 2015). Of all characterized MnLOXs, 13R-MnLOX is clearly the best studied enzyme. The manganese cofactor is assumed to catalyze the abstraction of the hydrogen atom similar to iron. Electron paramagnetic resonance (EPR) spectropscopy on MnLOXs further revealed that the manganese cofactor cycles between $\mathrm{Mn}$ (II) and $\mathrm{Mn}$ (III), in analogy to $\mathrm{Fe}(\mathrm{II})$ and $\mathrm{Fe}(\mathrm{III})$ in FeLOXs (Su et al., 2000). Although a crystal structure is not available yet, an octahedral coordination was also proposed for the manganese cofactor, since the amino acid residues of the coordination sphere are largely conserved. Only the C-terminal isoleucine is replaced by a valine residue in some MnLOXs (Supplemental Figure 4). However, not only the cofactor differs in MnLOXs, but also the specificity of the reaction. Most importantly, the antarafacial rule does not apply to MnLOXs. Instead, a suprafacial reaction was proposed in which the hydrogen abstraction and oxygen insertion must occur at the same side of the substrate (Hamberg et al., 1998). Mutational studies later suggested that Phe337 might be a determinant for the suprafacial reaction. Accordingly, replacement of this residue by an isoleucine resulted in a substantial decrease of catalytic activity, but also in an antarafacial hydrogen abstraction and oxygen insertion (Wennman et al., 2012). Additionally, Mn-LOXs can catalyze the oxygen insertion at the middle position of the pentadiene system, thus producing bis-allylic hydroperoxides that do not contain conjugated double bonds (Figure 11). On linoleic acid for instance, molecular oxygen is inserted at C11 to produce the bis-allylic 11S-hydroperoxy octadecadienoic acid (11S-HPODE). The formed 11S-HPODE is further isomerized to $13 R-H P O D E$ by MnLOX (Figure 11). This isomerization reaction is proposed to be catalyzed via deoxygenation and subsequent reoxygenation (Hamberg et al., 1998; Oliw et al., 2011).

\subsection{Bis-allylic hydroperoxides are also formed by an iron-containing LOX}

Although the formation of the bis-allylic product initially seemed to be restricted to MnLOXs, this assumption was disproved when CspLOX2 was characterized in 2010 (Andreou et al., 2010). This enzyme originates from the diazotrophic and unicellular bacterium Cyanothece $s p$. PCC8801, which harbors two LOX encoding genes, csplox1 and csplox2. Of these, csplox2 encodes for a LOX that was shown to produce the bis-allylic 11-HPODE as a major product of linoleic acid (Andreou et al., 2010). But in contrast to manganese-containing LOXs, the enzyme contains iron in the active site like prototypical LOXs from animals and plants. Furthermore, the reaction occurs in an antarafacial way and thus the $R$-isomer of 11-HPODE is formed after abstraction of the pro-S hydrogen (Figure 11 ). In fact, also the iron-containing $8 R$-LOX from $P$. homomalla has been shown to produce minor amounts ( $5 \%$ ) of the bis-allylic 10-hydroperoxy 
eicosatetraenoic acid (10-HETE) from arachidonic acid (Boutaud and Brash, 1999). Therefore, the metal cofactor is probably not the crucial determinant for the formation of the bis-allylic product, but rather unknown steric factors were suggested to direct oxygen to the middle position of the pentadiene moiety (Andreou et al., 2010).

\section{abstraction of the} pro-S hydrogen
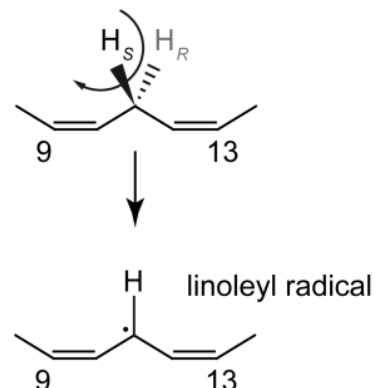

antarafacial oxygen insertion: CspLOX2

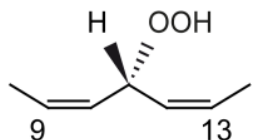

suprafacial

oxygen insertion: MnLOXs

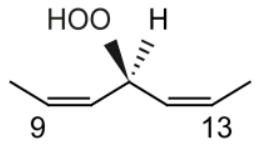

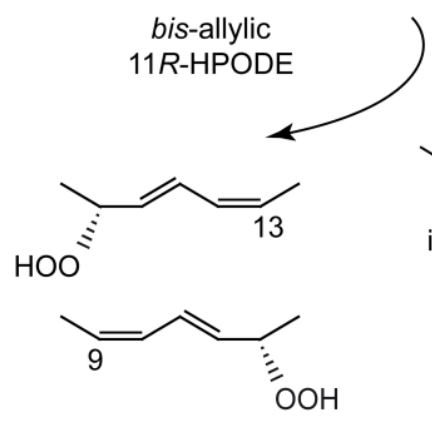

conjugated $9 R$ - and $13 S$-HPODE

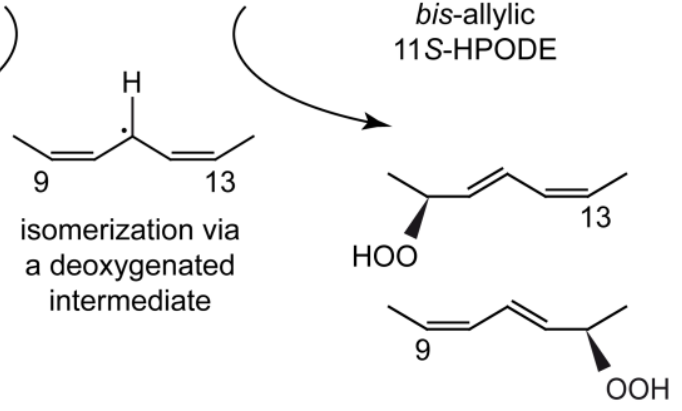

conjugated $9 S$ - and $13 R$-HPODE

Figure 11. Scheme of the formation and isomerization of the bis-allylic 11-HPODE by MnLOXs and CspLOX2. While Mn-LOXs catalyze the hydrogen abstraction and oxygen insertion at the same side of the substrate (suprafacial), CspLOX2 inserts dioxygen on the opposite side (antarafacial). In this scheme, only the reacting pentadiene system of linoleic acid is shown. 


\subsection{Theoretical concepts for specific oxygenation on one pentadiene}

As pointed out before, the proposed frame-shift and orientation models (Figure 10A and B) explain how a certain stereospecific hydrogen is targeted for abstraction (Egmond et al., 1972; Sloane et al., 1991). Nevertheless, information is still scarce for explaining how dioxygen can be inserted at different positions after abstraction of the same hydrogen atom. So far only the Gly/Ala switch (Figure 10C) provides a conceptual basis for directing the oxygen to different ends of the pentadiene after abstraction of the same hydrogen atom (Coffa and Brash, 2004). In the described model the switch only occurs between two positions on the pentadiene, while there are theoretically six reactive positions available for oxygen insertion if the bis-allylic ones are included. How the selection of one stereospecific product is achieved is still a matter of discussion.

Different theoretical mechanisms were considered for COX and LOX enzymes (Schneider et al., 2007). Both types of enzymes face similar challenges after hydrogen abstraction and use a similar chemistry in the dioxygenation of the fatty acid which is comparable to lipid autoxidation (Chapter 1.2). It should be noted that there is no evidence that oxygen binds covalently to the enzyme for example as $\mathrm{Fe}-\mathrm{O}_{2}$ or $\mathrm{Fe}-\mathrm{OOH}$ in LOXs. However, since free radical chemistry is involved, oxygen binding or activation is probably not necessary. Once the pentadienyl radical is formed, there is basically no energy barrier for the addition of molecular oxygen (Porter et al., 1995). This leaves the question open how such a specific oxygenation can be achieved.

When a carbon radical freely reacts with molecular oxygen, a peroxyl radical is formed. From free radical chemistry it is known that such peroxyl radicals can flip off and on the activated fatty acid. This reversible process is called " $\beta$-fragmentation" since the bond which is prone to break is found in the $\beta$-position to the radical center (Figure 12). The off reaction can be very fast, depending on the position of the oxygen addition. Peroxyl radicals at the end positions are considered to be rather stable with half-lives of milliseconds to seconds (Marnett, 1987; Roschek et al., 2006), whereas the off rate is about 4000 times faster at the bis-allylic position ( $>2 \mathrm{x}$ $10^{6} \mathrm{~s}^{-1}$ ) (Brash, 2000; Kitaguchi et al., 2005; Tallman et al., 2001). Hence, it is very difficult to trap the peroxyl radical at the middle position to form a bis-allylic hydroperoxide end-product. The reduction of the peroxyl radical by an appropriate hydrogen atom donor or an electron donor finally terminates the radical reaction. The availability and proper positioning of such a hydrogen/electron donor could thus influence the specificity of LOXs.

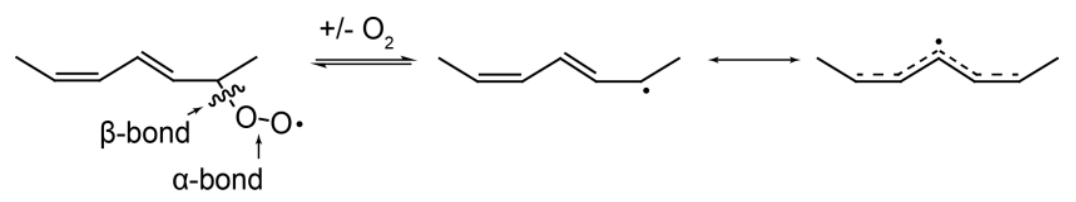

Figure 12. Reversibility of fatty acid peroxidation. Oxygen can flip off the activated fatty acid in a process called $\beta$ fragmentation. Addition of dioxygen to fatty acid radicals is therefore assumed to be reversible (Brash, 2000; Tallman et al., 2001). 
Based on these considerations, four potential mechanisms for oxygenation control were suggested by Schneider and colleagues which include (A) steric shielding, (B) oxygen channeling, (C) selective peroxyl radical trapping and (D) radical localization (Schneider et al., 2007) (Figure 13). Although these models present different concepts of oxygenation control, they may actually operate together and are not exclusive.

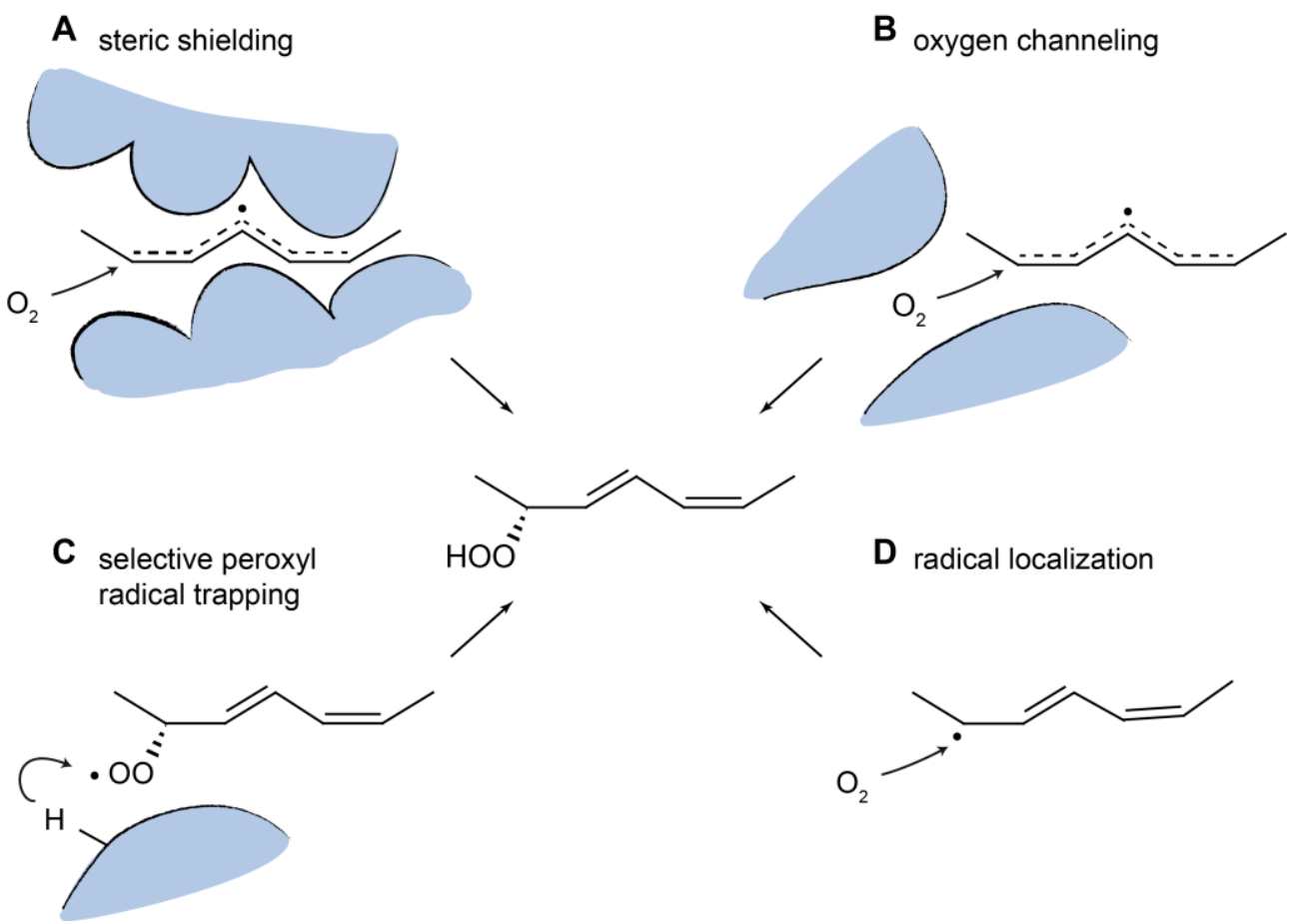

Figure 13. Four concepts of oxygenation control (Schneider et al., 2007). (A) Steric shielding by the surrounding amino acids might limit the oxygen access to the pentadiene, leaving only one position available. (B) An oxygen channel might transport oxygen directly to one specific position on the pentadiene. (C) A suitable hydrogen donor of the amino acid environment might trap the peroxyl radical, thereby preventing $\beta$-fragmentation at this position. (D) Torsions of the pentadienyl radical might lead to localization of the radical at one end of the pentadiene. This may result in oxygen insertion at this position.

A) Steric shielding can control the oxygen access to the reacting pentadiene by a tight binding in the active site (Figure 13A). Only one possible position of the pentadiene would be accessible to oxygen while all other positions would need to be blocked by the environment of the active site. Pockets in which oxygen may preferentially reside due to sufficient space or an increased solubility in hydrophobic environments may also play a role. It is almost certain that steric shielding is involved in controlling oxygenation, but it is not clear whether it is the sole mechanism (Schneider et al., 2007).

B) The existence of an oxygen channel that directs molecular oxygen precisely to one position and side of the activated fatty acid would be another option (Figure 13B). Although direct evidence is still missing, oxygen channels were proposed from the crystal structures of some LOXs as shown before (Figure 8). However, small oxygen 
molecules could in principle also enter through the substrate binding channel which also provides a hydrophobic environment (Schneider 2007).

C) The concept of selective peroxyl radical trapping is based on the assumption that the oxygenation specificity of the enzyme is not dependent on the oxygen addition, but trapping of the peroxyl radical (Figure 13C). Due to the reversibility of the oxygen addition ( $\beta$-fragmentation, Figure 12), trapping could prevent further on-off reactions of dioxygen. This would require a favorable positioning of a hydrogen atom donor to selectively trap peroxyl radicals at one position (Schneider et al., 2007).

D) The fourth model explains the specificity of the oxygen insertion through radical localization (Figure 13D). During autoxidation, the pentadienyl radical would adopt a planar structure since this displays the conformation of lowest energy. In a planar system, the radical can delocalize over all five carbon atoms. However, if the pentadiene is twisted out of plane by external forces, the unpaired electron becomes localized. Radical localization is well known from free radical chemistry, in which the pentadiene can be twisted out of plane by bulky substituents (Regenstein and Berndt, 1974; Schreiner and Berndt, 1974). This concept could in principle affect both, the regio- and stereospecificity of the oxygenation (Schneider et al., 2007).

\subsection{Cyanothece sp. harbors two LOX genes}

The two LOXs from Cyanothece sp., CspLOX1 and CspLOX2, were identified by sequence alignments and have been heterologously expressed in E. coli by Dr. Alexandra Andreou (Andreou et al., 2010; Newie et al., 2015). The only endogenous PUFA of Cyanothece sp. that could serve as potential LOX substrate is linoleic acid. Other PUFAs like $\alpha$-linolenic acid or arachidonic acid were not found in the fatty acid profile of Cyanothece sp. (Andreou et al., 2010). An initial biochemical characterization of both LOXs by Dr. Andreou revealed that CspLOX1 converts linoleic acid to 9R-HPODE and is therefore a 9R-LOX (Newie et al., 2015) while CspLOX2 is able to form the bis-allylic 11-HPODE and isomerize it to conjugated HPODEs (Andreou et al., 2010) (Figure 11). Both enzymes can insert dioxygen into phospholipids with bulky head groups. Therefore, a tail-first substrate orientation was proposed (Andreou et al., 2010; Newie et al., 2015). The crystal structures of both enzymes were solved by Dr. Piotr Neumann (Department of Molecular Structural Biology, University of Göttingen, Germany) before the beginning of this study. CspLOX1 was crystallized by Dr. Andreou and CspLOX2 was crystalized in own work. Despite a general biochemical characterization, both enzymes left open questions. The $\mathrm{N}$ terminal part of CspLOX1 for example exhibits a novel domain structure, but it was not clear whether it may fulfill a biological function. Furthermore, the question how a bis-allylic product can be formed by CspLOX2, while other iron-containing LOXs do not produce this product was still open. 


\subsection{Aim of this study}

The aim of this study was the detailed functional and structural characterization of both LOXs from Cyanothece sp. PCC8801, named CspLOX1 and CspLOX2. On the basis of the recently solved crystal structures of both enzymes, the contribution of different domains to a membrane binding function was analyzed in liposome assays with truncated versions of CspLOX1. Membrane binding domains of LOXs are particularly interesting as they might be involved in substrate acquisition directly from membranes.

Additionally, the CspLOX2 specificity for bis-allylic hydroperoxides was studied. First of all, the CspLOX2 reaction was analyzed in comparison to MnLOXs, as bis-allylic hydroperoxides have only been characterized as major products of MnLOXs and the iron-containing CspLOX2. It was therefore interesting to investigate the role of the metal cofactor in the active site by substitution of the iron cofactor of CspLOX2 with manganese and by EPR spectroscopy. Besides that, the main focus was to find a molecular basis for the formation of the bis-allylic 11-HPODE. The CspLOX2 crystal structure which is the first of a LOX with bis-allylic products allowed to address this question. Therefore, amino acids in the environment of the active site were exchanged and the effects on the oxygenation specificity were analyzed. To identify a conceptual basis, four different hypotheses were considered which are based on the models of Schneider and colleagues (Schneider et al., 2007). They include steric shielding, a specific oxygen channel, radical localization and peroxyl radical trapping as explanations for the formation of a bis-allylic product. In addition to biochemical data, a computational approach was applied to evaluate the relevance of these concepts for 11-HPODE formation by CspLOX2. 


\section{Materials and Methods}

\subsection{Materials}

\subsubsection{Chemicals}

All chemicals and solvents were obtained from Sigma-Aldrich (Munich, Germany) and Carl Roth \& Co. (Karlsruhe, Germany) unless stated otherwise. Agarose was from Biozym Scientific GmbH (Hessisch Oldendorf, Germany). Fatty acids were purchased from Sigma-Aldrich (Munich, Germany), Cayman (Ann Harbor, Great Britain) or Larodan (Linhamn, Sweden). HPLC-grade methanol, hexane and acetonitrile were from Acros Organics (Fisher Scientific, Waltham, MA, USA).

\subsubsection{Equipment}

Table 1. Machines and systems used in this study.

\begin{tabular}{ll} 
Machine or system & Manufacturer \\
\hline 1100 Series HPLC System & Agilent Technologies, Santa Clara, CA, USA \\
ÄKTA-FPLC & GE Healthcare, Chalfont St Giles, UK \\
ÄKTAprime plus & GE Healthcare, Chalfont St Giles, UK \\
CARY 100 Bio UV-vis Spectrophotometer & Varian Inc., Paolo Alto, CA, USA \\
Centrifuge 5417 R & Eppendorf, Hamburg, Germany \\
Centrifuge 5810 R & Eppendorf, Hamburg, Germany \\
Diana documentation system & Raytest, Straubenhardt, Germany \\
Elexsys E500 CW-EPR spectrometer & Bruker Biospin, Rheinstetten, Germany \\
Fluidizer & Microfluidics, Newton, MA, USA \\
Helium cryostat & Oxford Instruments, Abington, UK \\
IDA gel documentation system & Raytest, Straubenhardt, Germany \\
Lyophilizer & Leybold-Heraeus GmbH, Cologne, Germany \\
MAR345 image plate detector & Mar Research GmbH, Norderstedt, Germany \\
Mastercycler gradient & Eppendorf, Hamburg, Germany \\
Mastercycler personal & Eppendorf, Hamburg, Germany \\
MicroMax 007 & Rigaku, Tokio, Japan \\
Mini-PROTEAN3 Electrophoresis System & Bio-Rad, Hercules, CA, USA \\
Nanodrop 2000c & Thermo Fisher Scientific, Waltham, MA, USA \\
Optima 5500 DV & Perkin Elmer Precisely, Waltham, MA, USA \\
Oxygraph Plus System & Hansatech Instruments, Norfolk, UK \\
Sonifier Cell Disruptor B15 & Branson, Danbury, CT, USA \\
Sterile bench Prettl Telstar Bio II A & Telstar, Woerden, Netherlands \\
UHPLC 1290 Infinity & Agilent Technologies, Santa Clara, CA, USA \\
UV-table 312 nm & Raytest, Straubenhardt, Germany \\
&
\end{tabular}




\subsubsection{Kits, enzymes and commercial reagents}

Table 2. Commercial kits and reagents used in this study.

\begin{tabular}{ll} 
Kit, enzyme or marker & Manufacturer \\
\hline CloneJET PCR cloning kit & Thermo Fisher Scientific, Waltham, MA, USA \\
GeneRuler 1kb DNA ladder & Thermo Fisher Scientific, Waltham, MA, USA \\
Nucleospin extraction kit & Macherey Nagel, Düren, Germany \\
Nucleospin plasmid kit & Macherey Nagel, Düren, Germany \\
Pfu polymerase & Thermo Fisher Scientific, Waltham, MA, USA \\
Phusion polymerase & NEB, Ipswich, MA, USA \\
Protein molecular weight marker & Thermo Fisher Scientific, Waltham, MA, USA \\
Quick-Change mutagenesis Kit & Agilent Technologies, Santa Clara, CA, USA \\
Restriction endonucleases & Thermo Fisher Scientific, Waltham, MA, USA \\
T4 DNA ligase & Thermo Fisher Scientific, Waltham, MA, USA
\end{tabular}

\subsubsection{Data analysis and presentation}

For data analysis, statistics and visualization, different programs and tools were used which are listed in Table 3.

Table 3. Programs used in this study.

\begin{tabular}{|c|c|c|}
\hline Name & Purpose & Reference/Manufacturer \\
\hline APBS & $\begin{array}{l}\text { Generation of surface potential } \\
\text { maps }\end{array}$ & Baker et al., 2001 \\
\hline $\begin{array}{l}\text { Caver plugin for } \\
\text { PyMOL }\end{array}$ & Analysis of protein tunnels & CaverSoft s.r.o., Czech Republic \\
\hline CCP4 suite & Analysis of 3D-structures & Winn et al., 2011 \\
\hline Chemdraw & $\begin{array}{l}\text { Preparation of } 2 D \text { images of small } \\
\text { molecules }\end{array}$ & $\begin{array}{l}\text { CambridgeSoft, Waltham, MA, } \\
\text { USA }\end{array}$ \\
\hline Chemstation & Analysis of HPLC data & $\begin{array}{l}\text { Agilent Technologies, Santa Clara, } \\
\text { CA, USA }\end{array}$ \\
\hline Coot & $\begin{array}{l}\text { 3D-modeling, overlays of protein } \\
\text { structures }\end{array}$ & Emsley et al., 2010 \\
\hline Excel & Data analysis, statistics & Microsoft, Redmond, WA, USA \\
\hline Geneious & $\begin{array}{l}\text { Preparation of cloning strategies } \\
\text { and phylogenetic trees }\end{array}$ & Kearse et al., 2012 \\
\hline Illustrator & Preparation of all illustrations & Adobe, San José, CA, USA \\
\hline $\begin{array}{l}\text { Mass Hunter } \\
\text { B05.01 }\end{array}$ & $\begin{array}{l}\text { Acquisition and evaluation of } \\
\text { UHPLC QTOF MS data }\end{array}$ & $\begin{array}{l}\text { Agilent Technologies, Santa Clara, } \\
\text { CA, USA }\end{array}$ \\
\hline OriginPro & $\begin{array}{l}\text { Data analysis, preparation of } \\
\text { diagrams }\end{array}$ & $\begin{array}{l}\text { OriginLab, Northampton, MA } \\
\text { USA }\end{array}$ \\
\hline Phaser & Molecular Replacement & McCoy et al., 2007 \\
\hline Phenix & Structure refinement & Adams et al., 2010 \\
\hline Photoshop & Preparation of images & Adobe, San José, CA, USA \\
\hline
\end{tabular}




$\begin{array}{lll}\text { ProtParam } & \begin{array}{l}\text { Determination of protein } \\ \text { parameters }\end{array} & \begin{array}{l}\text { Walker, 2005 } \\ \text { PyMOL }\end{array} \\ \text { Visualization of protein structures } & \begin{array}{l}\text { DeLano Scientific LLC, } \\ \text { (Schrödinger) }\end{array} \\ \text { Xcalibur } & \text { Analysis of GC-MS data } & \begin{array}{l}\text { Thermo Scientific, Wilmington, } \\ \text { DE, USA }\end{array} \\ \text { XDS } & \begin{array}{l}\text { Indexing and integration of X-ray } \\ \text { diffraction data }\end{array} & \text { Kabsch, 2010 }\end{array}$

\subsubsection{Media}

The media used for cultivation of Escherichia coli are listed below:

Lysogeny Broth (LB) medium (Bertani, 1951)

\begin{tabular}{ll} 
Trypone & $10 \mathrm{~g}$ \\
Yeast extract & $5 \mathrm{~g}$ \\
$\mathrm{NaCl}$ & $5 \mathrm{~g}$ \\
\hline & Add $\mathrm{H}_{2} \mathrm{O}$ to $1 \mathrm{I}$
\end{tabular}

The medium was autoclaved at $120^{\circ} \mathrm{C}$ for $20 \mathrm{~min}$. For LB agar plates, $1.5 \%(\mathrm{w} / \mathrm{v})$ agar was added before autoclaving and the medium was chilled to about $60{ }^{\circ} \mathrm{C}$, before adding the appropriate antibiotic and preparation of the plates.

ZYP-5052 rich medium for auto-induction (Studier, 2005)

\begin{tabular}{ll}
$\mathrm{ZY}$ & $\sim 928 \mathrm{ml}$ \\
$1 \mathrm{M} \mathrm{MgSO}_{4}$ & $1 \mathrm{ml}$ \\
$1000 \times$ metals mix & $1 \mathrm{ml}$ \\
$50 \times 5052$ & $20 \mathrm{ml}$ \\
$20 \times \mathrm{NPS}$ & $50 \mathrm{ml}$ \\
\hline
\end{tabular}

PA-5052 defined medium for auto-induction (Studier, 2005)

\begin{tabular}{ll} 
Sterile water & $\sim 900 \mathrm{ml}$ \\
$1 \mathrm{M} \mathrm{MgSO}_{4}$ & $1 \mathrm{ml}$ \\
$50 \times 5052$ & $20 \mathrm{ml}$ \\
$20 \times$ NPS & $50 \mathrm{ml}$ \\
Methionine $(25 \mathrm{mg} / \mathrm{ml})$ & $8 \mathrm{ml}$ \\
17 amino acids $($ each $10 \mathrm{mg} / \mathrm{ml})$ & $20 \mathrm{ml}$ \\
\hline & 1 I final volume
\end{tabular}

The PA-5052 defined medium was supplemented with $50 \mu \mathrm{M} \mathrm{FeCl} 2$ or $\mathrm{MnCl}_{2}$ in order to obtain an iron- or manganese-containing version of CspLOX2.

For the auto-induction media these stock solutions had to be prepared individually and autoclaved for $20 \mathrm{~min}$ at $120^{\circ} \mathrm{C}$ : 
ZY

\begin{tabular}{ll}
$\mathrm{N}$-Z-amine AS & $10 \mathrm{~g}$ \\
Yeast extract & $5 \mathrm{~g}$ \\
\hline & Add $925 \mathrm{ml} \mathrm{H}_{2} \mathrm{O}$
\end{tabular}

\section{0x NPS}

\begin{tabular}{ll}
$\left(\mathrm{NH}_{4}\right)_{2} \mathrm{SO}_{4}$ & $66 \mathrm{~g}$ \\
$\mathrm{KH}_{2} \mathrm{PO}_{4}$ & $136 \mathrm{~g}$ \\
$\mathrm{Na}_{2} \mathrm{HPO}_{4}$ & $142 \mathrm{~g}$ \\
\hline & Add $900 \mathrm{ml} \mathrm{H} \mathrm{H}_{2} \mathrm{O}$ \\
\hline
\end{tabular}

\section{$50 \times 5052$}

\begin{tabular}{ll} 
Glycerol & $250 \mathrm{~g}$ \\
Glucose & $25 \mathrm{~g}$ \\
$\alpha$-lactose & $100 \mathrm{~g}$ \\
\hline & Add $730 \mathrm{ml} \mathrm{H}_{2} \mathrm{O}$
\end{tabular}

\section{0x metals mix}

The stock solutions of the individual metals were autoclaved individually, except for $0.1 \mathrm{M} \mathrm{FeCl}_{3}$ which was dissolved in $0.1 \mathrm{M} \mathrm{HCl}$.

\begin{tabular}{ll} 
Sterile $\mathrm{H}_{2} \mathrm{O}$ & $36 \mathrm{ml}$ \\
\hline $0.1 \mathrm{M} \mathrm{FeCl}_{3} \cdot 6 \mathrm{H}_{2} \mathrm{O}$ (in $\left.0.1 \mathrm{M} \mathrm{HCl}\right)$ & $50 \mathrm{ml}$ \\
$1 \mathrm{M} \mathrm{CaCl}_{2}$ & $2 \mathrm{ml}$ \\
$1 \mathrm{M} \mathrm{MnCl}_{2} \cdot 4 \mathrm{H}_{2} \mathrm{O}$ & $1 \mathrm{ml}$ \\
$1 \mathrm{M} \mathrm{ZnSO}_{4} \cdot 7 \mathrm{H}_{2} \mathrm{O}$ & $1 \mathrm{ml}$ \\
$0.2 \mathrm{M} \mathrm{CoCl}_{2} \cdot 6 \mathrm{H}_{2} \mathrm{O}$ & $1 \mathrm{ml}$ \\
$0.1 \mathrm{M} \mathrm{CuCl}_{2} \cdot 2 \mathrm{H}_{2} \mathrm{O}$ & $2 \mathrm{ml}$ \\
$0.2 \mathrm{M} \mathrm{NiCl}_{2} \cdot 6 \mathrm{H}_{2} \mathrm{O}$ & $1 \mathrm{ml}$ \\
$0.1 \mathrm{M} \mathrm{Na}_{2} \mathrm{MoO}_{4} \cdot 5 \mathrm{H}_{2} \mathrm{O}$ & $2 \mathrm{ml}$ \\
$0.1 \mathrm{M} \mathrm{Na}_{2} \mathrm{SeO}_{3} \cdot 5 \mathrm{H}_{2} \mathrm{O}$ & $2 \mathrm{ml}$ \\
$0.1 \mathrm{M} \mathrm{H}_{3} \mathrm{BO}_{3}$ & $2 \mathrm{ml}$
\end{tabular}

\section{7 amino acids $(10 \mathrm{ml} / \mathrm{ml})$}

To $90 \mathrm{ml}$ water in a beaker on a magnetic stirrer, $1 \mathrm{~g}$ each of the following 17 amino acids was added in this order: Na-Glu, Asp, Lys-HCl, Arg-HCl, His-HCl, Ala, Pro, Gly, Thr, Ser, Gln, Asn, Val, Leu, lle, Phe, Trp. The solution was sterilized by filtration ( $40 \mu \mathrm{m}$ pore size) and stored at $4{ }^{\circ} \mathrm{C}$.

\section{Antibiotics}

Depending on the antibiotic resistance of the bacteria, the media were supplemented with different antibiotics for selection. The final concentrations were as follows:

$\begin{array}{ll}\text { Carbenicillin } & 100 \mu \mathrm{g} / \mathrm{ml} \\ \text { Kanamycin } & 25 \mu \mathrm{g} / \mathrm{ml} \\ \text { Chloramphenicol } & 34 \mu \mathrm{g} / \mathrm{ml}\end{array}$




\subsubsection{Strains and vectors}

Vectors and E. coli strains are shown with their relevant features in Table 4 and Table 5.

Table 4. Strains used in this study.

\begin{tabular}{|c|c|c|}
\hline $\begin{array}{l}\text { Organism } \\
\text { and strain }\end{array}$ & Genotype & Supplier \\
\hline $\begin{array}{l}\text { E. coli } \\
\text { XL1-Blue }\end{array}$ & 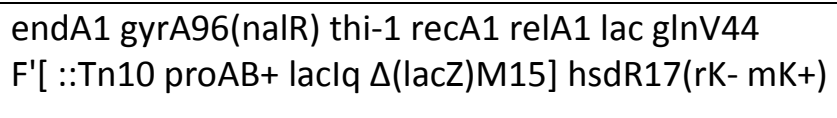 & $\begin{array}{l}\text { Agilent (Santa Clara, CA, } \\
\text { USA) }\end{array}$ \\
\hline $\begin{array}{l}\text { E. coli } \\
\text { BL21 Star } \\
\text { (DE3) }\end{array}$ & F- ompT hsdSB (rB-mB-) gal dcm rne131 (DE3) & $\begin{array}{l}\text { Thermo Fisher Scientific } \\
\text { (Waltham, MA, USA) }\end{array}$ \\
\hline
\end{tabular}

Table 5. Vectors used in this study.

\begin{tabular}{llll} 
Vector & Purpose & Important features & Supplier \\
\hline pJET2.1/blunt & Cloning vector & Ampicillin resistance & $\begin{array}{l}\text { Thermo Fisher Scientific } \\
\text { (Waltham, MA, USA) }\end{array}$ \\
\hline pET28a & Expression vector & $\begin{array}{l}\text { Kanamycin resistance, } \\
\text { T7 promoter }\end{array}$ & $\begin{array}{l}\text { Novagen (Darmstadt, } \\
\text { Deutschland) }\end{array}$
\end{tabular}

LOX genes were cloned into pET28a in a way that the hexahistidine Tag (6xHis-Tag) is fused to the $\mathrm{N}$-terminal part of the protein.

\subsection{Molecular biology methods}

If not mentioned otherwise, all molecular and microbiological methods were performed as described by Ausubel et al., 1993 or Sambrook et al., 1989.

\subsubsection{Plasmid DNA isolation}

Plasmid DNA was isolated with the commercially available NucleoSpin Plasmid-Kit (MachereyNagel, Düren, Germany) following the provided protocol. Overnight cultures of the cells containing the target plasmid were cultivated at $37^{\circ} \mathrm{C}$ in $4 \mathrm{ml} \mathrm{LB}$ medium. The isolation process is based on alkaline lysis (Bimboim and Doly, 1979) which causes denaturation of both chromosomal and plasmid DNA. After acidification, plasmid DNA is renatured, but not chromosomal DNA which is subsequently precipitated by centrifugation. The target plasmid is bound to the silica matrix and can finally be eluted after washing. The isolated plasmid DNA was used for sequencing, site-directed mutagenesis, DNA restriction or transformation.

\subsubsection{Preparation of chemically competent E. coli cells and transformation of plasmid DNA}

For the preparation of chemically competent $E$. coli cells (Inoue et al., 1990), $500 \mathrm{ml}$ LB medium were inoculated with 5-10 colonies from a LB plate and distributed to four 1 I flasks. The cultures 
were incubated at $37^{\circ} \mathrm{C}$ for $2 \mathrm{~h}$ under shaking and subsequently transferred to $20-25^{\circ} \mathrm{C}$. When an $\mathrm{OD}_{600}$ of $0.45-0.75$ was reached, the cells were transferred to sterile $50 \mathrm{ml}$ tubes and incubated on ice for $10 \mathrm{~min}$. After sedimentation of the cells by centrifugation at $4^{\circ} \mathrm{C}$ for $10 \mathrm{~min}$ at $1000 \mathrm{~g}$, the medium was discarded completely and the cell pellet was resuspended in $16 \mathrm{ml}$ ice cold TFB buffer ( $10 \mathrm{mM}$ PIPES, $15 \mathrm{mM} \mathrm{CaCl}_{2}, 250 \mathrm{mM} \mathrm{KCl}, 55 \mathrm{mM} \mathrm{MnCl}_{2}, \mathrm{pH}$ 6.7) per $50 \mathrm{ml}$ tube. The incubation on ice and centrifugation was repeated. Finally, all sedimented cells were carefully resuspended in a total volume of $20 \mathrm{ml}$ TFB buffer. A volume of $3 \mathrm{ml}$ dimethyl sulfoxide was added as cryoprotectant. After another incubation for $10 \mathrm{~min}$ on ice, the chemically competent cells were distributed to aliquots (200-500 $\mu \mathrm{l})$, frozen in liquid nitrogen and stored at $-80^{\circ} \mathrm{C}$.

For transformation of plasmid DNA, the competent cells were carefully thawed on ice and mixed with 1-10 $\mu$ l DNA per $100 \mu$ of cells. After an incubation for 20 min on ice, a heat shock was performed at $42{ }^{\circ} \mathrm{C}$ for $1 \mathrm{~min}$ (Mandel and Higa, 1970). After brief chilling of the transformed cells on ice, $900 \mu \mathrm{LB}$ was added and the cells were allowed to recover for $1 \mathrm{~h}$ at $37^{\circ} \mathrm{C}$ while shaking. The cells were collected by centrifugation for $1 \mathrm{~min}$ at $4000 \mathrm{~g}$ and the supernatant was discarded. The cells were resuspended in the residual drop and spread on selective LB agar plates containing the appropriate antibiotic.

\subsubsection{Amplification of DNA fragments by polymerase chain reaction}

Polymerase chain reaction (PCR) is an efficient technique to selectively amplify a specific part of a double stranded DNA in vitro (Mullis et al., 1992). First, the double stranded DNA is denatured into single strands at temperatures around $95^{\circ} \mathrm{C}$. Sequence specific single stranded primers then bind to their complementary sequences in the denatured DNA at lower temperatures $\left(\sim 55^{\circ} \mathrm{C}\right)$. Elongation of the primers is then catalyzed in $5^{\prime}$ to $3^{\prime}$ direction by a DNA-polymerase. Using thermostable enzymes allows a cycling of this reaction and hence an exponential amplification of the DNA fragment located between both primers.

\section{Colony PCR}

This PCR technique allows a quick analysis of recombinant clones grown on selective agar plates (Woodman, 2008). A very small amount of cell material is transferred from a single colony to a PCR vial. The selected colonies were marked and transferred to a fresh selective agar plate. During the initial denaturation, cells are disrupted and the DNA becomes accessible for amplification. A PCR mix was prepared on ice as follows and added to the cells.

$1 \mu \mathrm{l} \quad$ forward primer $(10 \mu \mathrm{M})$

$1 \mu \mathrm{l}$ reverse primer $(10 \mu \mathrm{M})$

$1 \mu \mathrm{l}$ dNTPs (10 $\mu \mathrm{M}$ each)

$4 \mu \mathrm{l}$ GoTaq polymerase buffer

$0.1 \mu \mathrm{l}$ GoTaq polymerase (Promega, Fitchburg, USA)

$18 \mu \mathrm{l} \quad \mathrm{H}_{2} \mathrm{O}$

$25 \mu \mathrm{l}$ Final volume 
For amplification of the DNA fragment, a thermal cycler (Mastercycler Personal, Eppendorf, Hamburg, Germany) was used with the following program, whereby cycling was performed from the denaturation to the elongation step:

\begin{tabular}{lrll} 
Initial denaturation & $5 \mathrm{~min}$ & $95^{\circ} \mathrm{C}$ & \\
\hline Denaturation & $30 \mathrm{~s}$ & $95^{\circ} \mathrm{C}$ & \\
Primer annealing & $30 \mathrm{~s}$ & $55^{\circ} \mathrm{C}$ & 30 cycles \\
Elongation & $1 \mathrm{~min} / \mathrm{kb}$ & $72{ }^{\circ} \mathrm{C}$ & \\
\hline Final Elongation & $10 \mathrm{~min}$ & $72{ }^{\circ} \mathrm{C}$ &
\end{tabular}

\section{Truncation of genes}

In order to create truncated versions of LOX genes, primers were designed to bind to the edges of the desired fragment. In addition to the complementary sequence, short overhangs with suitable restriction sites were added to the $3^{\prime}$ and $5^{\prime}$ end to allow a cloning into pET28a later on. The PCR reaction was prepared as follows:

$1 \mu \mathrm{l}$ plasmid DNA

$1 \mu \mathrm{l}$ forward primer $(10 \mu \mathrm{M})$

$1 \mu \mathrm{l}$ reverse primer $(10 \mu \mathrm{M})$

$1 \mu \mathrm{l}$ dNTPs $(10 \mu \mathrm{M}$ each)

$10 \mu \mathrm{l} \quad 5 \mathrm{xHF}$ buffer

$0.5 \mu \mathrm{l}$ Phusion polymerase (NEB, Ipswich, MA, USA)

$35.5 \mu \mathrm{l} \quad \mathrm{H}_{2} \mathrm{O}$

$50 \mu \mathrm{l}$ Final volume

The DNA fragment was amplified with the following PCR program:

\begin{tabular}{lrrr} 
Initial denaturation & $30 \mathrm{~s}$ & $98^{\circ} \mathrm{C}$ & \\
\hline Denaturation & $30 \mathrm{~s}$ & $98^{\circ} \mathrm{C}$ & \\
Primer annealing & $1 \mathrm{~min}$ & $55^{\circ} \mathrm{C}$ & $25 \mathrm{cycles}$ \\
Elongation & $30 \mathrm{~s} / \mathrm{kb}$ & $72{ }^{\circ} \mathrm{C}$ & \\
\hline Final Elongation & $10 \mathrm{~min}$ & $72{ }^{\circ} \mathrm{C}$ &
\end{tabular}

The fragment was then ligated into pJET 1.2/blunt according to the CloneJET ${ }^{\mathrm{TM}}$ PCR Cloning Kit manual (Thermo Fisher Scientific, Waltham, MA, USA). Using the inserted restriction sites, the fragment could be cloned into the final expression vector $\mathrm{pET} 28 \mathrm{a}$.

\section{QuikChange PCR for site directed mutagenesis of single base pairs}

Single or multiple base pairs can be efficiently substituted by a PCR method that is based on the commercially available QuikChange II Site-Directed Mutagenesis Kit from Agilent Technologies (Santa Clara, CA, USA). Primers were designed to be complementary to each other and cover the sequence that is targeted for the mutation. These nucleotides were exchanged in the primers. Additionally, the primers should contain a $3^{\prime}$-overhang of at least 8 base pairs to minimize the formation of primer dimers. If possible, a silent mutation was introduced in proximity to the desired mutation in order to insert or delete restriction sites for an easy selection process of the clones. 
The PCR reaction contained the following components:

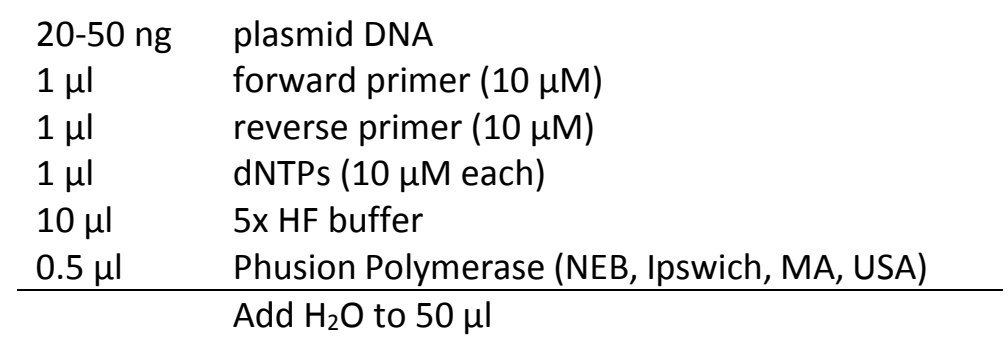

The conditions during the thermal cycling were as follows:

\begin{tabular}{lrll} 
Initial denaturation & $30 \mathrm{~s}$ & $98^{\circ} \mathrm{C}$ & \\
\hline Denaturation & $30 \mathrm{~s}$ & $98^{\circ} \mathrm{C}$ & \\
Primer annealing & $1 \mathrm{~min}$ & $55^{\circ} \mathrm{C}$ & 18 cycles \\
Elongation & $30 \mathrm{~s} / \mathrm{kb}$ & $72^{\circ} \mathrm{C}$ & \\
\hline Final Elongation & $10 \mathrm{~min}$ & $72^{\circ} \mathrm{C}$ &
\end{tabular}

The methylated template DNA was subsequently digested by addition of $1 \mu$ of the restriction enzyme Dpnl and incubation for $1 \mathrm{~h}$ at $37{ }^{\circ} \mathrm{C}$. Finally, $10 \mu \mathrm{l}$ of the reaction were directly transformed into $E$. coli XL1-Blue. Positive clones could be selected by testing for the additional or deleted restriction site and DNA sequencing.

\subsubsection{DNA sequence analysis}

The accuracy of the constructed plasmids was confirmed by DNA sequencing. The samples were prepared by mixing $5 \mu \mathrm{l}$ of isolated plasmid DNA (80-100 $\mathrm{ng} / \mu \mathrm{l})$ with $5 \mu \mathrm{l}$ of a suitable primer $(5 \mu \mathrm{M})$. Analysis was performed by the company GATC (Cologne, Germany). The applied technique is based on Sanger sequencing (Sanger et al., 1977).

\subsubsection{DNA precipitation}

DNA precipitation is a method to purify and concentrate nucleic acids. The solubility of the polar DNA was decreased by addition of $1 / 10$ volume of sodium acetate and 2.5 volumes of pure ice cold ethanol to the aqueous DNA solution. The mixture was incubated for at least $1 \mathrm{~h}$ at $-20^{\circ} \mathrm{C}$ and centrifuged at $22,000 \mathrm{~g}$ for $30 \mathrm{~min}$ at $4{ }^{\circ} \mathrm{C}$. The sometimes barely visible DNA pellet was carefully rinsed with $70 \%$ Ethanol and centrifuged for another $10 \mathrm{~min}$. After complete removal of the supernatant, the DNA pellet was dried and resuspended in the desired amount of water.

\subsubsection{Ligation of compatible DNA fragments}

In order to ligate DNA fragments with compatible overhangs created by DNA restriction digestion, a T4 DNA ligase was used (Thermo Fisher Scientific, Waltham, MA, USA). After attachment of the cohesive overhangs due to Watson-Crick base pairing, the T4 DNA ligase catalyzes the formation of phospodiester bonds between junxtaposed 5'-phosphate and 3'hydroxyl termini in double-stranded DNA (Weiss and Richardson, 1967). The enzyme can also ligate blunt DNA fragments without overhangs. The reaction was set up as follows: 
$1 \mu \mathrm{l} \quad$ T4 DNA Ligase buffer

$0.5 \mu \mathrm{l}$ T4 DNA Ligase $(5 \mathrm{U} / \mu \mathrm{l})$

$6 \mu \mathrm{l}$ Insert

$3 \mu \mathrm{l}$ Vector

For ligation of PCR fragments into the pJET2.1/blunt cloning vector, the commercial kit was used as indicated by the manufacturer (Thermo Fisher Scientific, Waltham, MA, USA).

\subsubsection{Agarose gel electrophoresis}

DNA fragments were separated according to their size by agarose gel electrophoresis (Aaij and Borst, 1972). An agarose solution of $1 \%(\mathrm{w} / \mathrm{v})$ was prepared in $1 \times$ Tris-acetate-EDTA (TAE) buffer and solved by heating in a microwave oven. The solution was poured into a gel caster and a comb of suitable size was used to form the sample pockets. The samples were mixed with 0.25 volumes of DNA loading dye and loaded onto the solidified agarose gel. A marker ( $1 \mathrm{~kb}$ gene ruler, Thermo Fisher Scientific, Waltham, MA, USA) was loaded in parallel to determine the size of individual DNA fragments. Separation of the negatively charged DNA fragments by size was achieved by applying an electric potential of $120 \mathrm{~V}$ in a chamber filled with TAE buffer. After separation, the gel was stained for $15 \mathrm{~min}$ in an ethidium bromide solution $(2 \mu \mathrm{g} / \mathrm{ml})$. Since the fluorescent dye ethidium bromide intercalates with DNA, its fluorescence signal can be used to visualize DNA bands in a UV transilluminator (IDA Raytest, Straubenhardt, Germany).

TAE buffer (50x) $2 \mathrm{M}$ Tris, $1 \mathrm{M}$ acetic acid, $0.05 \mathrm{M}$ ethylendiaminetetraacetic acid (EDTA)

DNA loading dye $100 \mathrm{mM}$ EDTA, $0.1 \%(\mathrm{w} / \mathrm{v})$ sodium dodecyl sulfate (SDS), $40 \%(\mathrm{w} / \mathrm{v})$ glycerol, $0.025(\mathrm{w} / \mathrm{v})$ bromphenol blue, $0.025(\mathrm{w} / \mathrm{v})$ xylencyanol blue

\subsubsection{Restriction of DNA}

Double stranded DNA can specifically be cut at target sequences by restriction enzymes (Boyer, 1971). Reactions were set up in volumes of 20-30 $\mu$ l with conventional restriction enzymes from Thermo Fisher Scientific (Waltham, MA, USA) according to the manufacturer's instructions. The DNA fragments could subsequently be analyzed by agarose gel electrophoresis.

\subsubsection{Isolation of DNA from agarose gels}

In order to purify DNA fragments resulting from PCR or restriction digestion, samples were first separated by agarose gel electrophoresis and stained with ethidium bromide as described above. The DNA fragment was checked for its size and cut quickly out of the gel. UV-light with a relatively low intensity was used to avoid mutations. For the isolation of the DNA fragments from the gel, the Nucleospin Purification Kit (Macherey-Nagel, Düren, Germany) was used according to the manufacturer's instructions. The DNA was finally eluted in $20 \mu \mathrm{l} \mathrm{H}_{2} \mathrm{O}$ and stored at $-20^{\circ} \mathrm{C}$. 


\subsection{Protein expression and purification}

\subsubsection{Cultivation of $E$. coli for protein expression}

For high yields of a heterologously expressed protein, larger amounts of cell material had to be produced. Precultures were prepared in LB medium and inoculated with 5-10 E. coli colonies containing the pET28a expression plasmid. After overnight incubation at $37^{\circ} \mathrm{C}$ on a shaker, high cell densities of $\mathrm{OD}_{600}$ of 5-7 were reached. The protein expression was then performed in ZYP5052 rich medium for auto-induction (Studier, 2005) that was supplemented with $25 \mu \mathrm{g} / \mathrm{ml}$ kanamycin to ensure that only cells containing the pET28a plasmid will grow. For sufficient aeration of the cultures, 1 or 2 I Erlenmeyer flasks were filled to approximately $1 / 4$ of their maximal capacity. The medium was inoculated with $1 / 100$ volume of the preculture, resulting in a starting $\mathrm{OD}_{600}$ of about 0.05 . Main cultures were cultivated at $28^{\circ} \mathrm{C}$ and $200 \mathrm{rpm}$ for $16 \mathrm{~h}$. Since the medium contains both, glucose and lactose, the cells first metabolize glucose as a preferred carbon source. As long as glucose is present in the medium, the lac promoter is switched off, as glucose inhibits the import of lactose. During that phase, cells grow and proliferate, but do not express protein. When glucose is used up (after about $6 \mathrm{~h}$ ), lactose is taken up which induces the lac promoter and in turn the expression of the T7 RNA-polymerase. This polymerase causes the expression of the target protein for about $10 \mathrm{~h}$. Finally, cells were harvested at 2,900 $\mathrm{g}$ for 20 min (Beckman Coulter centrifuge, Brea, CA, USA), transferred to $50 \mathrm{ml}$ tubes, frozen in liquid nitrogen and stored at $-20{ }^{\circ} \mathrm{C}$.

\subsubsection{Cell lysis}

Frozen cell pellets were resuspended to a cell density of $1 \mathrm{~g}$ cells per 3-4 $\mathrm{ml}$ buffer A (see below) supplemented with $0.1 \mathrm{mg} / \mathrm{ml}$ lysozyme to break the cell walls and $0.2 \mathrm{mM}$ phenylmethylsulfonyl fluoride to inhibit proteases. The homogeneity of the solution was ensured by filtering the suspension through a syringe. After $30 \mathrm{~min}$ incubation on ice, the cells were disrupted at 80 Psi for 3 cycles with a fluidizer (Microfluidics, Newton, MA, USA). For smaller amounts of less than $30 \mathrm{ml}$, cells were disrupted by sonication (Sonifier Cell Disruptor B15, Branson, Danbury, CT, USA) in 3 cycles of 1 min with $50 \%$ intensity and pulsed signal. To prevent heating and thus degradation of proteins, cells were always kept on ice. After cell disruption, insoluble particles and cell debris were precipitated by centrifugation at $27,000 \mathrm{~g}$ at $4{ }^{\circ} \mathrm{C}$ for 20 min (Beckman Coulter centrifuge, Brea, CA, USA). The supernatant which is also referred to as cleared lysate was then applied to an affinity chromatography column. 


\subsubsection{Protein purification by immobilized metal ion affinity chromatography}

An easy method to purify tagged proteins from a mixture is the immobilized metal ion chromatography (IMAC). It utilizes the ability of complex-forming amino acid residues, especially histidine, to coordinate metal ions. Therefore, a protein with a polyhistidine-tag (His-Tag) will specifically interact with nickel, cobalt or copper ions immobilized on a column and will be retained. As most naturally occurring proteins do not have such a high affinity for metal ions, they will not bind to the column and can be washed off.

The proteins purified in this study carry a His-Tag on the $\mathrm{N}$-terminus accessible at the protein surface. The cleared lysate was loaded on a pre-packed $5 \mathrm{ml} \mathrm{His-Trap} \mathrm{HP}$ column containing $\mathrm{Ni}^{2+}-$ sepharose (GE Healthcare Life Science, Chalfont St Giles, Great Britain) that was previously equilibrated with buffer $A$. The flow rate of $0.5-1 \mathrm{ml} / \mathrm{min}$ was controlled using an ÄKTAprime plus system (GE Healthcare Life Science, Chalfont St Giles, Great Britain). Unspecifically bound proteins were washed from the column with butter $A$ and also with a mixture of $97 \%$ buffer $A$ and $3 \%$ buffer $B$. The specifically retained proteins were finally eluted from the column during a $50 \mathrm{ml}$ gradient when the concentration of buffer B was increased from $3 \%$ to $100 \%$. Buffer B contains imidazole that competes with the His-Tag for binding to the metal ions and hence liberates the target protein. The eluate could be concentrated by centrifugation in Spin-X UF concentrators (Corning, Corning, NY, USA) at $4{ }^{\circ} \mathrm{C}$ and $4000 \mathrm{rpm}$ (Centrifuge $5810 \mathrm{R}$, Eppendorf, Hamburg, Germany).

Buffer A $\quad 50 \mathrm{mM}$ Tris- $\mathrm{HCl} \mathrm{pH} 8,100 \mathrm{mM} \mathrm{NaCl}$

Buffer B $50 \mathrm{mM}$ Tris- $\mathrm{HCl}$ pH 8, $100 \mathrm{mM} \mathrm{NaCl}, 500 \mathrm{mM}$ imidazole

\subsubsection{Size-exclusion chromatography}

To further purify proteins and remove undesirable protein aggregates, size-exclusion chromatography (SEC) was applied. This method, also referred to as gel filtration, simultaneously allows a buffer exchange and removal of imidazole from previous purification steps. The column is packed with a porous matrix that allows molecules to diffuse in and out depending on their size. Smaller molecules can diffuse into smaller pores and will thus stay longer on the column, while larger molecules are unable to move into the pores. They will therefore elute first as the buffer continuously passes through the column.

The concentrated protein solution of less than $4 \mathrm{ml}$ was injected to a pre-equilibrated HiLoad 26/60 Superdex $200 \mathrm{pg}$ column with a total volume of $300 \mathrm{ml}$ (GE Healthcare Life Science, Chalfont St Giles, Great Britain) containing sepharose material. The flow rate of the SEC buffer (50 mM Tris-HCl pH 8, $100 \mathrm{mM} \mathrm{NaCl}$ ) was adjusted to 1-2 ml/min with an ÄKTA-FPLC system (GE Healthcare Life Science, Chalfont St Giles, Great Britain). Fractions of 5-8 ml were collected after the void volume of $100 \mathrm{ml}$ had passed through the column. 


\subsubsection{Metal cofactor substitution by expression in minimal medium}

For the exchange of the metal cofactor, CspLOX2 was expressed in PA-5052 defined autoinduction medium (Studier, 2005). The preculture, which was prepared in the complex LB medium, was used to inoculate the expression culture with 1/100 of the final volume. The medium contains a defined mix of amino acids instead of yeast extract and NZ-amines to minimize a contamination with manganese or iron. The medium was supplemented with kanamycin and either $50 \mu \mathrm{M} \mathrm{MnCl}_{2}$ or $50 \mu \mathrm{M} \mathrm{FeCl}_{2}$, depending on the metal center that should be incorporated. Traces of LB medium that were transferred during inoculation should cover the needs for further trace elements. After growing the cultures at $28^{\circ} \mathrm{C}$ for $16 \mathrm{~h}$, the cells were harvested, lysed using the fluidizer and cleared from the cell debris as described above. For the purification via IMAC and SEC, the buffers were treated with Chelex 100 (Bio Rad, Hercules, CA, USA) before use to eliminate additional transition metals from the solution. As far as possible, the usage of plastic ware was preferred over glass ware during purification, since iron can also be released from glass materials.

\subsection{Protein analysis and biochemical characterization}

\subsubsection{Quantification of purified protein}

The amount of protein in solution was determined by two different techniques depending on the purity and presence of additional components in the protein solution. While the colorimetric Bradford assay (Bradford, 1976) tolerates imidazole and detects different kinds of proteins, the protein determination at $280 \mathrm{~nm}$ (Gill and Von Hippel, 1989) is more reproducible, but only suitable for pure protein solutions without compounds that absorb at $280 \mathrm{~nm}$.

\section{Bradford protein analysis}

The Bradford protein assay is a colorimetric method that is based on the absorbance shift of Coomassie Brilliant Blue G-250 from $465 \mathrm{~nm}$ to $595 \mathrm{~nm}$ upon binding to protein (Bradford, 1976). For the assay, 5-10 $\mu$ l protein solution were added to $1 \mathrm{ml}$ Bradford reagent and incubated at $23{ }^{\circ} \mathrm{C}$ for 20 min. A calibration curve with 0-12 $\mu \mathrm{g}$ BSA was always prepared in parallel. The protein concentration was analyzed at $595 \mathrm{~nm}$ with a spectrophotometer (Varian Inc., Paolo Alto, CA, USA). The absorbance of the solution to be analyzed should be in the range of the calibration curve. Otherwise, the protein solution had to be diluted. The linear regression of the values from the calibration curve was used to determine the concentration of the protein solution. The analysis was always performed in triplicates. 


\section{Determination of the protein content at $280 \mathrm{~nm}$}

Protein concentrations could also be determined as absorbance at $280 \mathrm{~nm}$ with a NanoDrop 2000c (Thermo Scientific, Wilmington, DE, USA), which is a fast and simple method that requires only a volume of $1.5 \mu \mathrm{l}$ protein solution per measurement. Another advantage is that a large concentration range of $0.1-400 \mathrm{mg} / \mathrm{ml}$ can be directly measured without dilutions. With the molecular weight and the theoretical absorption coefficient which could be determined with ProtParam (Walker, 2005), the protein concentration could be reproducibly determined.

\subsubsection{Protein analysis by polyacrylamide gel electrophoresis}

Polyacrylamide gel electrophoresis with sodium dodecyl sulfate (SDS-PAGE) is a widely used analytical method for separation of proteins according to their molecular weights (Laemmli, 1970). The method was used to determine the expression level under different cultivation conditions or to analyze the purity of a protein solution at different steps of the purification. For the analysis of protein expression levels, in general $0.5 / O D_{600} \mathrm{ml}$ of the culture were harvested for $1 \mathrm{~min}$ at 14,000 $\mathrm{g}$ and resuspended in $100 \mu \mathrm{l}$ x SDS sample buffer. For the analysis of purified proteins, 5-10 $\mu$ g protein were diluted to $7.5 \mu \mathrm{l}$ and supplemented with $2.5 \mu \mathrm{l}$ of the $4 \mathrm{x}$ SDS sample buffer. The proteins were denatured at $95^{\circ} \mathrm{C}$ for $3 \mathrm{~min}$, centrifuged for $1 \mathrm{~min}$ and $10 \mu \mathrm{l}$ of the samples were loaded on an SDS polyacrylamide gel.

Proteins were electrophoretically separated in $10 \%$ discontinuous gels under denaturing conditions in Tris/glycine buffer (25 mM Tris, $192 \mathrm{mM}$ glycine, $0.1 \%$ (w/v) SDS). The gels were prepared and run in systems from BioRad (Hercules, CA, USA). The mixture for six gels was as follows, whereby ammonium persulfate (APS) and Tetramethylethylenediamine (TEMED) were added last, as they trigger the polymerization of the gel in a radical reaction:

\begin{tabular}{lll} 
& $\begin{array}{l}\text { Separating } \\
\text { gel }\end{array}$ & $\begin{array}{l}\text { Stacking } \\
\text { gel }\end{array}$ \\
\hline $\mathrm{H}_{2} \mathrm{O}$ & $9.51 \mathrm{ml}$ & $4.47 \mathrm{ml}$ \\
$1.5 \mathrm{M}$ Tris-HCl pH 8.8, 0.4 \% SDS & $6 \mathrm{ml}$ & - \\
$0.5 \mathrm{M} \mathrm{Tris-HCl} \mathrm{pH} \mathrm{6.8,} \mathrm{0.4} \mathrm{\%} \mathrm{SDS}$ & - & $1.88 \mathrm{ml}$ \\
$30 \%(w / v)$ acylamide/bis-acrylamide (37.5:1) (Roth, Carlsruhe) & $8.1 \mathrm{ml}$ & $1.05 \mathrm{ml}$ \\
$25 \%(w / v)$ APS & $96 \mu l$ & $30 \mu l$ \\
TEMED & $24 \mu \mathrm{l}$ & $7.5 \mu l$ \\
\hline Total volume & $\sim 24 \mathrm{ml}$ & $\sim 7.5 \mathrm{ml}$ \\
Volume per gel & $\sim 3.7 \mathrm{ml}$ & $\sim 1 \mathrm{ml}$
\end{tabular}

4x SDS sample buffer

$200 \mathrm{mM}$ Tris-HCl pH 6.8, 400 mM 1,4-dithiothreitol (DTT), $8 \%$ (w/v) SDS, $0.4 \%(\mathrm{w} / \mathrm{v})$ bromophenol blue, $40 \%$ (v/v) glycerol

The gels could be stored at $4{ }^{\circ} \mathrm{C}$ in wet paper towels for up to 2 weeks. If the sample pockets were prepared with a 15 well-comb, a sample volume of up to $20 \mu \mathrm{l}$ could be loaded per well. To determine the size of the separated proteins, a protein marker (Roti-Mark Standard, Roth, 
Karlsruhe, Germany) was loaded in parallel. A current of $30 \mathrm{~mA}$ per gel was applied in the electrophoresis chamber (power supply E838, Consort, Turnhout, Belgium) for 45-60 min until the bromophenol blue front reached the bottom of the gel.

After separation, the proteins in the gel were stained with a blue Commassie Brilliant Blue dye which can visualize microgram amounts of protein (Meyer and Lamberts, 1965). Proteins are stained rather unspecifically, since the dye interacts with basic and aromatic side chains of amino acids. The gels were carefully removed from the glass plates and stained in Coomassie Brilliant blue stain for $60 \mathrm{~min}$ and subsequently destained in an aqueous solution containing $40 \%(\mathrm{v} / \mathrm{v})$ ethanol and $10 \%(\mathrm{v} / \mathrm{v})$ acetic acid until the blue protein bands stand out from the transparent background.

\section{Commassie Brilliant Blue stain \\ $0.2 \%(\mathrm{w} / \mathrm{v})$ Brilliant Blue R250, 40\% (v/v) ethanol, $10 \%(\mathrm{v} / \mathrm{v})$ acetic acid}

\subsubsection{Kinetic measurements}

\section{Absorbance at $234 \mathrm{~nm}$}

The LOX activity could be determined as absorbance increase at $234 \mathrm{~nm}$ with a Cary 100 Bio spectrophotometer (Varian, Agilent Technologies, Santa Clara, CA, USA) (Graff et al., 1990). This technique, however, detects only the formation of conjugated double bonds, since they absorb at this wavelength. Bis-allylic lipoxygenation products cannot be detected due to the lack of conjugated double bonds. Fatty acid substrates were diluted to 5-150 $\mu \mathrm{M}$ in $200 \mathrm{mM}$ sodium borate buffer $\mathrm{pH} 9$ and preheated to $30^{\circ} \mathrm{C}$. The reaction was started by addition of 3-15 $\mu \mathrm{g}$ enzyme to $1 \mathrm{ml}$ substrate solution. The turnover number $k_{\text {cat }}$ was calculated from the slope of the reaction and the enzyme concentration as follows:

$$
k_{\text {cat }}[1 / s]=\frac{\Delta E}{c_{\text {enzyme }} \cdot \varepsilon_{234} \cdot d}
$$

$$
\begin{array}{ll}
\Delta E & =\text { change of extinction at } 234 \mathrm{~nm} \text { per second } \\
c_{\text {enzyme }} & =\text { enzyme concentration in the assay in } \mathrm{M} \\
\varepsilon_{234} & =\text { molar extinction coefficient at } 234 \mathrm{~nm}=2.5 \cdot 10^{4} \mathrm{M}^{-1} \mathrm{~cm}^{-1} \\
d \quad & =\text { length of the cuvette in } \mathrm{cm}
\end{array}
$$

\section{Oxygen electrode}

To determine the total lipoxygenation activity including the formation of bis-allylic hyroperoxides, the consumption of oxygen was measured as a function of time with an oxygen electrode (Oxygraph Plus System, Hansatech Instruments, Norfolk, UK). First, the electrode was calibrated from the maximum oxygen concentration at $30^{\circ} \mathrm{C}$ in water to $0 \mathrm{nmol} / \mathrm{ml}$ oxygen which was established with sodium dithionite. Similar to the photometric assay, the substrates were diluted to different concentrations in $200 \mathrm{mM}$ sodium borate buffer $\mathrm{pH} 9$ and the reaction was 
started by addition of 3-15 $\mu \mathrm{g}$ enzyme to $1 \mathrm{ml}$ preheated $\left(30^{\circ} \mathrm{C}\right)$ substrate solution. Since $1 \mathrm{~mol}$ $\mathrm{O}_{2}$ is consumed per mol of hydroperoxy product, the turnover number can be calculated as follows:

$$
k_{\text {cat }}[1 / s]=\frac{\Delta c_{\text {oxygen }}}{c_{\text {enzyme }}}
$$

$\Delta c_{\text {oxygen }}=$ change of oxygen concentration $\left(\mu \mathrm{M} \mathrm{O}_{2} / \mathrm{s}\right)$

$c_{\text {enzyme }}=$ enzyme concentration in the assay in $\mu \mathrm{M}$

\section{Determination of the inhibitory constant $K_{1}$}

To determine the inhibitory effect of tetraethylene glycol monooctyl ether (C8E4), CspLOX2 was incubated with $100 \mu \mathrm{M}$ of linoleic acid and different amounts of C8E4 in $200 \mathrm{mM}$ sodium borate buffer. The inhibitory constant $K_{1}$ of C8E4 was determined as follows:

$$
v=v_{\text {rest }}+\frac{v_{I} \cdot K_{I}}{K_{I}+c_{I}}
$$

$v \quad=$ reaction rate

$v_{\text {rest }} \quad=$ remaining reaction rate

$v_{I} \quad=$ initial reaction rate without inhibitor

$K_{I} \quad=$ inhibitory constant

$c_{I} \quad=$ concentration of inhibitor

\subsubsection{Analysis of the metal content by inductively coupled plasma atomic emission} spectroscopy

For the determination of the cofactor occupancy, inductively coupled plasma atomic emission spectroscopy (ICP-AES) was applied. This technique can detect small amounts of trace metals with detection limits up to $2 \mu \mathrm{g} / \mathrm{l}$. The inductively coupled plasma is used to generate excited ions and atoms that emit electromagnetic radiation at wavelengths which are characteristic for specific elements. This electromagnetic radiation can be detected. The intensity of the emitted radiation is indicative of a particular element in the sample. As the method is very sensitive and shows only few interferences with different chemicals, it is suitable for measuring the metal content of enzymes.

To avoid metal contaminations, protein samples were purified by size exclusion chromatography with a buffer (50 mM Tris/ $\mathrm{HCl}$ pH 8, $100 \mathrm{mM} \mathrm{NaCl}$ ) treated with Chelex100 (BioRad, Hercules, CA, USA). This buffer was also used as blank and for dilution of the protein samples to 5-25 $\mu \mathrm{M}$ in a final volume of $3 \mathrm{ml}$. The samples were measured at an Optima 5500 DV (Perkin Elmer Precisely) by Uta Nüsse-Hahne and Dr. Dietrich Hertel from the Department of Plant Ecology and 
Ecosystem Research (University of Göttingen, Germany). The measurement was performed in triplicates with relative standard deviations of in general less than $5 \%$.

\subsubsection{Electron paramagnetic resonance}

Electron paramagnetic resonance (EPR) spectroscopy is based on the interaction of electromagnetic radiation with magnetic moments which are induced by the spin of the electrons. The method utilizes microwave radiation to excite unpaired electrons in the presence of a static magnetic field. It is therefore limited to paramagnetic substances, such as radicals or certain transition metals and is a suitable technique to analyze the metal cofactor and its oxidation states in LOXs.

Continuous wave EPR spectra were recorded on a Bruker Elexsys E500 CW-EPR spectrometer at 9.4 GHz (X-band) continuous wave equipped with a standard Bruker X-band ER4119-SHQE cavity and a liquid helium cryostat (Oxford Instruments, Abington, UK). The experiments were conducted by Dr. Müge Kasanmaschef (Electron Paramagnetic Resonance Spectroscopy Group, Max Planck Institute for Biophysical Chemistry, Göttingen, Germany). CspLOX2 and linoleic acid were mixed to final concentrations of $30 \mu \mathrm{M}$ enzyme and $700 \mu \mathrm{M}$ substrate, respectively. For the preparation of other samples, CspLOX2 and HPODE were mixed to a final concentration of $30 \mu \mathrm{M}$ enzyme and $70 \mu \mathrm{M}$ HPODE, respectively. The reactions were allowed to proceed at room temperature for $1 \mathrm{~min}$ and were subsequently manually freeze-quenched in liquid nitrogen. The parameters for all recorded EPR spectra were as follows: $\mathrm{T}=10 \mathrm{~K}$, microwave power $=1 \mathrm{~mW}$, conversion time $=80 \mathrm{~ms}$, modulation amplitude $=5 \mathrm{G}$, modulation frequency $=100 \mathrm{kHz}, 40 \mathrm{~min}$, signal averaging.

\subsection{Analysis of CspLOX2 products}

\subsubsection{Extraction of hydroperoxy products}

For the analysis of lipoxygenation products, the formed hydroperoxides had to be extracted from an aqueous solution in preparation for subsequent chromatographic analysis. For individual analysis of products from single reactions, $1 \mathrm{ml}$ of $100 \mu \mathrm{M}$ linoleic acid in $200 \mathrm{mM}$ sodium borate buffer $\mathrm{pH} 9$ was converted with $15 \mu \mathrm{g}$ of enzyme. Since the unusual 11hydroperoxide from linoleic acid is only transiently formed by CspLOX2, the reaction was usually monitored with a spectrophotometer at $234 \mathrm{~nm}$ and stopped after the first reaction phase by transferring $500 \mu \mathrm{l}$ of the reaction mix to $500 \mu \mathrm{l}$ of diethylether in a $2 \mathrm{ml}$ reaction tube. After brief but thorough mixing, the reaction tubes were placed on ice. When the phases were separated, the upper organic phase was transferred to a new reaction vial and the solvent was evaporated under a nitrogen stream. The remaining lipid film was resuspended in a suitable solvent for HPLC analysis on the same day. To obtain reproducible results, it was important that all steps were done quickly. For the preparation of larger amounts of 11-HPODE, $1 \mathrm{mg}$ linoleic 
acid was converted with $15 \mu \mathrm{g} \operatorname{CspLOX} 2$ at $25{ }^{\circ} \mathrm{C}$ for $8 \mathrm{~min}$. For some experiments, the hydroperoxides had to be reduced to the hydroxides. In that case, sodium borohydride was added to the sample after the reaction. After incubation for $2 \mathrm{~min}$ at $23^{\circ} \mathrm{C}$, the fatty acid derivatives were extracted with diethylether.

\subsubsection{High-performance liquid chromatography analysis of lipoxygenation products}

High-performance liquid chromatography (HPLC) is a method to separate and analyze different lipid species in a mixture. Different types of stationary and mobile phases are used to achieve the separation of analytes, while a pump transports the sample and solvent through the column. As interactions with the column depend on physicochemical properties of the analytes, they will elute after different retention times. An installed diode array detector (DAD) records UV/Vis spectra during elution. Information about the spectrum and retention time together with the area of the peak allows an identification and quantification of the analytes. Absorption at $234 \mathrm{~nm}$ was recorded for detection of conjugated double bonds during all chromatographic steps, while absorbance at $202 \mathrm{~nm}$ was used for detection of the fatty acid substrate and bisallylic products. For identification of the compounds, authentic standards were run in parallel.

\section{Reversed phase HPLC}

11-HPODE could be separated from conjugated hydroperoxides and the fatty acid substrate by reversed-phase (RP)-HPLC using a Nucleosil 120-5 $\mathrm{C}_{18}$ column $(250 \times 2.1 \mathrm{~mm}, 5 \mu \mathrm{m}$ particle size, Macherey-Nagel, Düren, Germany). This chromatographic method uses a hydrophobic stationary phase which results in a stronger adsorption of hydrophobic molecules to the column. Hence, more hydrophilic molecules elute first.

The extracted lipids were dissolved in $100 \mu \mathrm{l}$ of the RP-HPLC solvent A. The samples were transferred to HPLC vials and $80 \mu \mathrm{l}$ were injected for analysis. Separation was performed with a solvent system of solvent $A$, acetonitrile/water/acetic acid (50:50:0.05, v/v/v) and solvent $B$, acetonitrile/acetic acid (100:0.05, v/v) using the following gradient as described (Andreou et al., 2010):

$\begin{array}{rrrl}0 \mathrm{~min} & 20 \% \mathrm{~B} & 80 \% \mathrm{~A} & \text { Flow rate: } 0.18 \mathrm{ml} / \mathrm{min} \\ 10 \mathrm{~min} & 20 \% \mathrm{~B} & 80 \% \mathrm{~A} & \text { Flow rate: } 0.18 \mathrm{ml} / \mathrm{min} \\ 15 \mathrm{~min} & 100 \% \mathrm{~B} & 0 \% \mathrm{~A} & \text { Flow rate: } 0.18 \mathrm{ml} / \mathrm{min} \\ 17 \mathrm{~min} & 100 \% \mathrm{~B} & 0 \% \mathrm{~A} & \text { Flow rate: } 0.36 \mathrm{ml} / \mathrm{min} \\ 25 \mathrm{~min} & 100 \% \mathrm{~B} & 0 \% \mathrm{~A} & \text { Flow rate: } 0.36 \mathrm{ml} / \mathrm{min} \\ 27 \mathrm{~min} & 20 \% \mathrm{~B} & 80 \% \mathrm{~A} & \text { Flow rate: } 0.36 \mathrm{ml} / \mathrm{min} \\ 30 \mathrm{~min} & 20 \% \mathrm{~B} & 80 \% \mathrm{~A} & \text { Flow rate: } 0.36 \mathrm{ml} / \mathrm{min}\end{array}$

\section{Radio RP-HPLC}

In addition to the DAD detector, a scintillation detector was used for the detection of the radiation emitted from isotope labeled compounds. [1- $\left.\mathrm{C}^{14}\right]$-linoleic acid was used as substrate for the enzymatic conversion with LOXs. The generated products were separated by RP-HPLC 
and detected with a DAD and a scintillation detector. From the ratio of the UV absorbance at $202 \mathrm{~nm}$ (11-HPODE) or $234 \mathrm{~nm}$ (9- and 13 HPODE) and the radio signal, relative scaling factors were determined (Supplemental Figure 2). The ratio was determined for six HPLC runs with different concentrations. The scaling factors $(S F)$ were obtained from the slope $(m)$ of the linear regression curve as follows (exemplified for 9-HPODE):

$$
S F_{9-H P O D E}=\frac{3 \cdot m_{9-H P O D E}}{m_{9-H P O D E}+m_{11-H P O D E}+m_{13-H P O D E}}
$$

These scaling factors were later used for a quantification of HPODEs without [1-C $\left.\mathrm{C}^{14}\right]$-labeled substrate by multiplying these factors with the determined peak areas determined at $202 \mathrm{~nm}$ and $234 \mathrm{~nm}$, respectively.

\section{RP-HPLC-MS}

The bis-allylic product could also be analyzed and quantified by RP-HPLC coupled to mass spectrometry (MS) for detection of the analytes. The method was based on (Brodhun et al., 2009). Separation was achieved on a EC250/2 Nucleodur 100-5 $C_{18}$ column ( $250 \times 2.1 \mathrm{~mm}, 5 \mu \mathrm{m}$ particle size, Macherey-Nagel, Düren, Germany) with a solvent system of solvent $A$, acetonitrile/water/acetic acid (40:60:0.1, v/v/v) and solvent B, acetonitrile/acetic acid (100:0.1, $\mathrm{v} / \mathrm{v}$ ) with the following elution gradient:

$\begin{array}{rrrl}0 \mathrm{~min} & 20 \% \mathrm{~B} & 80 \% \mathrm{~A} & \text { Flow rate: } 0.2 \mathrm{ml} / \mathrm{min} \\ 10 \mathrm{~min} & 20 \% \mathrm{~B} & 80 \% \mathrm{~A} & \text { Flow rate: } 0.2 \mathrm{ml} / \mathrm{min} \\ 30 \mathrm{~min} & 100 \% \mathrm{~B} & 0 \% \mathrm{~A} & \text { Flow rate: } 0.2 \mathrm{ml} / \mathrm{min} \\ 35 \mathrm{~min} & 100 \% \mathrm{~B} & 0 \% \mathrm{~A} & \text { Flow rate: } 0.3 \mathrm{ml} / \mathrm{min} \\ 40 \mathrm{~min} & 100 \% \mathrm{~B} & 0 \% \mathrm{~A} & \text { Flow rate: } 0.3 \mathrm{ml} / \mathrm{min} \\ 44.5 \mathrm{~min} & 20 \% \mathrm{~B} & 80 \% \mathrm{~A} & \text { Flow rate: } 0.3 \mathrm{ml} / \mathrm{min} \\ 45 \mathrm{~min} & 20 \% \mathrm{~B} & 80 \% \mathrm{~A} & \text { Flow rate: } 0.2 \mathrm{ml} / \mathrm{min}\end{array}$

The eluting substances were analyzed by ion trap mass spectrometry (Thermo Finnigan LCQ ion trap mass spectrometer, San Jose, CA, USA). Negatively charged ions were generated by electron spray ionization. The capillary voltage was $27 \mathrm{kV}$ and the capillary temperature was $300^{\circ} \mathrm{C}$. For tandem MS analysis, the collision energy was $1 \mathrm{~V}$.

\section{Straight phase HPLC}

Positional isomers of hydro(pero)xy fatty acid were separated by straight phase (SP)-HPLC on a Zorbax Rx-SIL column (150 × 2.1 mm, $5 \mu \mathrm{m}$ particle size, Agilent Technologies, Santa Clara, CA, USA) as described (Andreou et al., 2010). This method separates analytes based on their affinity for a stationary phase with a polar surface. The compounds are eluted with a solvent system of $n$-hexane/2-propanol/acetic acid (100:1:0.05, v/v/v) at a flow rate of $0.2 \mathrm{ml} / \mathrm{min}$. 


\section{Chiral phase HPLC}

Enantiomers of the conjugated hydroxy fatty acids (9-HODE and 13-HODE) could be analyzed by chiral phase (CP)-HPLC using a Chiralcel OD-H column $(150 \times 2.1 \mathrm{~mm}, 5 \mu \mathrm{m}$ particle size, Daicel, VWR, Radnor, PA, USA) with a solvent system of $n$-hexane/2-propanol/acetic acid (100:5:0.05, $\mathrm{v} / \mathrm{v} / \mathrm{v}$ ) at a flow rate of $0.1 \mathrm{ml} / \mathrm{min}$ (Andreou et al., 2010).

\section{Analysis of stereochemistry of 11-HPODE}

For the CP-HPLC analysis of the bis-allylic 11-HPODE according to (Andreou et al., 2010), the products had to be derivatized to the hydroxy octadecadienoic acid methyl esters (HODE-Me). Therefore sodium borohydride was added to $1 \mathrm{ml}$ of the reaction mixture to reduce the fatty acid hydroperoxides to hydroxides. Diethylether was added for extraction of all hydrophobic analytes and the extract was dried under a nitrogen stream. Fatty acid hydroxides were subsequently methylated by addition of $400 \mu$ l methanol and $6 \mu$ l diazomethane (Figure 14).

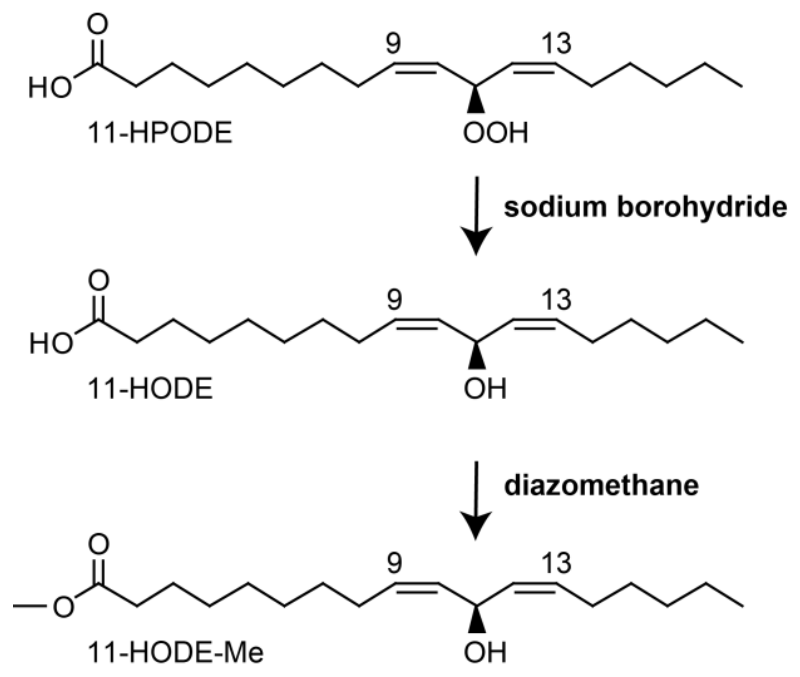

Figure 14. Derivatization of hydroperoxides to hydroxides and their methyl esters.

After $30 \mathrm{~min}$, the reaction was stopped with $2.5 \mu \mathrm{l}$ 1:10 (v/v) acetic acid. The solvent was evaporated under a stream of nitrogen and 11-HODE-Me was separated from the conjugated products by SP-HPLC. 11-HODE-Me was collected, dried and subjected to CP-HPLC analysis. A separation of 11-HODE-Me enantiomers was achieved at a flow rate of $0.1 \mathrm{ml} / \mathrm{min}$ in a solvent system of $n$-hexane/2-propanol/acetic acid (100:0.3:0.05, v/v/v). 11S- and 11R-HODE-Me eluted after around 70-90 min from the column.

\subsubsection{Fragmentation analysis by UHPLC-ESI-QTOF-MS/MS}

For fragmentation studies, the samples were analysed by Ultra High Performance Liquid Chromatography (UHPLC, 1290 Infinity, Agilent Technologies, Santa Clara, CA, USA) coupled with a 6540 UHD Accurate-Mass Quatupole Time-of-Flight (QTOF) LC-MS instrument with Agilent Dual Jet Stream Technology as electrospray ion source (ESI, Agilent Technologies, Santa Clara, 
CA, USA) by Dr. Kirstin Feußner (Department for Plant Biochemistry, University of Göttingen, Germany). For LC separation, an ACQUITY UPLC ${ }^{\circledR}$ HSS T3 column $(2.1 \times 100$ mm, $1.8 \mu \mathrm{m}$ particle size, Waters Corporation, Milford, USA) was used at $40{ }^{\circ} \mathrm{C}$ and a flow rate of $0.5 \mathrm{ml} / \mathrm{min}$. The following gradient program was performed with a solvent system of $A$, water/formic acid (100:0.1, v/v) and B, acetonitrile/formic acid (100:0.1, v/v).

$\begin{array}{rrl}0 \min & 99 \% \mathrm{~A} & 1 \% \mathrm{~B} \\ 0.5 \min & 80 \% \mathrm{~A} & 20 \% \mathrm{~B} \\ 3 \min & 0 \% \mathrm{~A} & 100 \% \mathrm{~B} \\ 8 \min & 0 \% \mathrm{~A} & 100 \% \mathrm{~B} \\ 10 \min & 0 \% \mathrm{~A} & 100 \% \mathrm{~B} \\ 10.1 \min & 99 \% \mathrm{~A} & 1 \% \mathrm{~B} \\ 14 \min & 80 \% \mathrm{~A} & 1 \% \mathrm{~B}\end{array}$

The QTOF MS instrument was operated in the positive or the negative ionization mode with a detection frequency of $2 \mathrm{GHz}$ and a mass range of $m / z 50-1700$. The source conditions were as following: gas temperature: $250^{\circ} \mathrm{C}$; drying gas flow: $8 \mathrm{l} / \mathrm{min}$; nebulizer pressure: 35 psi; sheath gas temperature: $300^{\circ} \mathrm{C}$; sheath gas flow: $8 \mathrm{I} / \mathrm{min}$; VCap voltage: $3.0 \mathrm{kV}$; nozzle voltage: $200 \mathrm{~V}$; fragmentor voltage: $120 \mathrm{~V}$. For MS/MS fragmentation experiments the precursor ions of 9HODE, 11-HODE as well as 13-HODE ([M-H] 295.2267) were fragmented by a collision cell energy of $28 \mathrm{eV}$. The methyl esters of $11 R$ - and 11S-HODE were analyzed in the positive ionization mode. They were detected as $\left[\mathrm{M}-\mathrm{H}_{2} \mathrm{O}+\mathrm{H}\right]^{+} 293.2477$ and fragmented by a collision cell energy of $15 \mathrm{eV}$. The UHPLC QTOF MS system was operated under Mass Hunter B05.01 (Agilent Technologies, Santa Clara, CA, USA). Data were acquired by Mass Hunter Workstation Acquisition software B.05.01 and analyzed by Mass Hunter Qualitative Analysis B.06.00.

\subsection{Analysis of liposome binding ability}

\subsubsection{Liposome preparation}

Liposomes are small spherical vesicles that consist of a lipid bilayer surrounding an aqueous solution. They are thus similar to biological membranes. For the preparation of these vesicles, glass tubes and the extruder were carefully rinsed with pure undenatured ethanol before use. The phospholipid for the liposome preparation, 1,2-dipalmitoyl-sn-glycero-3-phosphocholine (Sigma-Aldrich, Munich, Germany), was dissolved in chloroform to a concentration of $1 \mathrm{mg} / \mathrm{ml}$ as described by Bigay and Antonny, 2005. The solvent was evaporated under a stream of nitrogen above the transition temperature at $55^{\circ} \mathrm{C}$ in a water bath. As a result, only a very thin lipid layer remained on the surface of the glass tube. Remnants of the solvent were removed by lyophilization at $-20^{\circ} \mathrm{C}$ overnight. The thin lipid layer was dissolved in sodium phosphate buffer (100 mM Na $2 \mathrm{HPO}_{4} / \mathrm{NaH}_{2} \mathrm{PO}_{4}, \mathrm{pH}$ 7) to a lipid concentration of $10 \mathrm{mM}$. The solution was repeatedly heated to $60^{\circ} \mathrm{C}$ and vigorously mixed. Subsequently, three cycles of freezing at $-80{ }^{\circ} \mathrm{C}$ and heating to $60^{\circ} \mathrm{C}$ followed. Finally, the liposomes were prepared with an extruder (Avanti 
Polar Lipids, Alabaster, AL, USA). The extruder was preheated to $60^{\circ} \mathrm{C}$ and assembled according to the manufacturer's instructions. The liposomes were passed 41 times through the polycarbonate membrane with the extruder placed on a heating plate at $60^{\circ} \mathrm{C}$. The odd number of passages ensured that lipids which never passed through the membrane are excluded from the final liposome solution.

\subsubsection{Flotation analysis}

The ability of proteins to bind to liposomes can be tested in a so-called flotation experiment (Bigay and Antonny, 2005). For this assay, $50 \mu \mathrm{l}$ of a protein solution with a concentration of $1 \mathrm{mg} / \mathrm{ml}$ was mixed with $100 \mu$ l of liposomes. After an incubation of $90 \mathrm{~min}$ at room temperature to allow binding to the vesicles, $100 \mu \mathrm{l}$ of a $75 \%(\mathrm{w} / \mathrm{v}$ ) sucrose stock solution (in $100 \mathrm{mM}$ $\mathrm{Na}_{2} \mathrm{HPO}_{4} / \mathrm{NaH}_{2} \mathrm{PO}_{4}, \mathrm{pH} 7$ ) was added to the mixture to yield a final sucrose concentration of $30 \%(\mathrm{w} / \mathrm{v})$. The samples were filled into $2 \mathrm{ml}$ ultracentrifugation vials. Subsequently, $200 \mu \mathrm{l}$ of a $25 \%$ (w/v) sucrose solution (in $100 \mathrm{mM} \mathrm{Na} \mathrm{HPO}_{4} / \mathrm{NaH}_{2} \mathrm{PO}_{4} \mathrm{pH}$ 7) were carefully layered onto the samples. Finally, $50 \mu \mathrm{l}$ of $100 \mathrm{mM} \mathrm{Na} \mathrm{HPO}_{4} / \mathrm{NaH}_{2} \mathrm{PO}_{4} \mathrm{pH} 7$ were filled on top. The samples were then centrifuged at $210,000 \mathrm{~g}$ in a TLS 55 rotor (Beckman Coulter, Brea, CA, USA) for $90 \mathrm{~min}$. During this ultracentrifugation, the liposomes floated up due to a lower density compared to the sucrose solution and moved to the top fraction with all proteins bound to them. After centrifugation, the fractions were carefully separated with a Hamilton syringe from the top to the bottom fraction $($ top $=100 \mu \mathrm{l}$, middle $=150 \mu \mathrm{l}$, bottom $=250 \mu \mathrm{l}$ ). Proteins were precipitated by addition of $1 / 4$ volume of ice cold $30(\mathrm{w} / \mathrm{v})$ trichloroacetic acid and overnight incubation on ice. Samples were centrifuged at $4{ }^{\circ} \mathrm{C}$ for $30 \mathrm{~min}$ at $20,000 \mathrm{~g}$ and the protein pellet was delipidated with acetone and finally washed in $100 \mathrm{mM} \mathrm{Na} 2 \mathrm{HPO}_{4} / \mathrm{NaH}_{2} \mathrm{PO}_{4} \mathrm{pH}$ 7. The samples were resuspended in $30 \mu \mathrm{l} 1 \mathrm{x}$ SDS sample buffer (see Chapter 2.4.2) and $7 \mu \mathrm{l}$ thereof were loaded on a gel for SDS-PAGE analysis.

\subsection{Protein crystallography}

To obtain X-ray structures of proteins, protein crystals have to be grown first. Crystal screens were prepared in hanging drop vapor diffusion crystallization plates (EasyXtal 15-Well Tool, Qiagen, Venlo, Netherlands or VDX Plate, Hampton Research, Aliso Viejo, CA, USA) with CspLOX2 purified by IMAC and SEC. Protein concentrations of $3.5-5 \mathrm{mg} / \mathrm{ml}$ in $25 \mathrm{mM}$ Tris- $\mathrm{HCl}$ pH 8, $50 \mathrm{mM}$ $\mathrm{NaCl}$ resulted in best crystals. To prevent overnucleation, the protein was centrifuged at $14,000 \mathrm{~g}$ for $30 \mathrm{~min}$ at $4{ }^{\circ} \mathrm{C}$ prior to pipetting the crystallization screens. Best crystals were obtained when reservoir solutions containing around $12.5 \%$ PEG 4000, 0-8 \% glycerol, 600 mM $\mathrm{NaCl}$ and 0.1 M MES/imidazole pH 6.1-6.8 were used. In general, about 200-300 $\mu$ l were used as reservoir and 1-2 $\mu \mathrm{l}$ of the protein was mixed with equal amounts of reservoir to prepare the hanging drops. The crystallization plates were sealed (in case of VDX Plates with vacuum grease) and incubated at $20^{\circ} \mathrm{C}$. Crystals usually appeared within $24 \mathrm{~h}$ and grew to a size of $0.10 \times 0.03 \times$ $0.03 \mathrm{~mm}$ within 3-7 days. Crystals were cryoprotected by transferring them for a few seconds to 
20 \% PEG4000, 17 \% glycerol, 600 mM NaCl, 0.1 M MES/imidazole pH 6.25 or 15 \% PEG 4000, $20 \%$ glycerol, $600 \mathrm{mM} \mathrm{NaCl}, 0.1 \mathrm{M} \mathrm{MES} /$ imidazole pH 6.25. The crystals were quickly cooled in liquid nitrogen.

For anaerobic crystallization in the laboratory of Prof. Dr. Marcia Newcomer (Department of Biological Sciences, Louisiana State University, Baton Rouge, USA), all buffers and solutions were transferred to an anaerobic chamber and allowed to equilibrate for at least $24 \mathrm{~h}$. The anaerobic chamber was filled with a mixture of nitrogen and hydrogen gas $\left(97 \% \mathrm{~N}_{2}\right.$ and $3 \% \mathrm{H}_{2}$, respectively). A catalyzer that transforms oxygen and hydrogen to water always kept the oxygen concentration close to $0 \mathrm{ppm}$. The protein was purified at ambient oxygen levels and transferred to the anaerobic chamber in small aliquots of up to $50 \mu$ l on an ice pack to keep it cold until pipetting. Crystallization screens were prepared inside the chamber. When crystals were grown to a proper size in the anaerobic atmosphere, liquid nitrogen was brought into the chamber. Oxygen levels rose temporarily to $20-40 \mathrm{ppm}$ but then stabilized at $0 \mathrm{ppm}$ oxygen again. The crystallization wells were not opened before the oxygen reached $0 \mathrm{ppm}$. Crystals were mounted on a loop and quickly plunged and kept the liquid nitrogen, before taking them out of the anaerobic chamber.

\subsubsection{Manipulation of protein crystals}

\section{Soaking and cocrystallization}

In order to study the reaction mechanism of an enzyme, it is a common technique to soak crystals with substrates, substrate-analogs, products or inhibitors. Soaking solutions were based on the reservoir or cryoprotectant solution and contained 0.1-2 $\mathrm{mM}$ of the respective substrate or substrate-analog. Crystals were transferred to the soaking solution and incubated for 3$180 \mathrm{~min}$. If necessary, crystals were cryoprotected and then transferred to liquid nitrogen.

As an alternative, it is sometimes possible to cocrystallize the enzyme with the substrate. The protein was either pre-mixed with the substrate and then combined with the reservoir solution or the substrate was added to the reservoir before mixing it with the protein. Usually, a molar ratio of $1: 1$ to $100: 1$ of substrate to protein was used. To improve the solubility of the hydrophobic substrates, ethanol was in some cases added to a final concentration of 1-5 \% (v/v).

\section{Xenon derivatization}

Xenon derivatization can be used to identify oxygen channels in protein structures (Luna et al., 2008). Due to similar properties, xenon may reside in the cavities where gases like dioxygen may bind. The experiments were performed with the help of Prof. Dr. Newcomer. CspLOX2 crystals were transferred into the single crystal xenon chamber (Hampton Research, Aliso Viejo, CA, USA) coated with reservoir solution to prevent crystal dehydration. A xenon pressure of 75-110 Psi was applied for $5 \mathrm{~min}$. After a slow pressure release during $30 \mathrm{~s}$, crystals were quickly frozen in liquid nitrogen. 


\subsubsection{Data collection and structure determination}

The quality of crystals could be tested at $100 \mathrm{~K}$ on the microfocus rotating anode MicroMax-007 (Rigaku, Tokyo, Japan) equipped with a MAR345 image plate detector (Marresearch GmbH, Norderstedt, Germany). For higher resolution datasets, crystals were measured at synchrotron beam lines (Desy, Hamburg, Germany; European Synchrotron Radiation Facility (ESRF), Grenoble, France; Advanced Photon Source (APS), Chicago, USA) by Dr. Piotr Neumann (Department of Molecular Structural Biology, University of Göttingen, Germany) or Dr. David Neau (Department of Chemistry and Chemical Biology, Cornell University, Argonne, USA). Diffraction data were indexed and integrated with the XDS program (Kabsch, 2010). The wild type (wt) structure of CspLOX2 was used as template for Molecular Replacement using the Phaser program (McCoy et al., 2007). The model was further manually improved in Coot (Emsley et al., 2010) and refined with Phenix (Adams et al., 2010). Prior to use in structural refinement, randomly selected $5 \%$ of the reflections were set aside for the calculation of Rfree as a quality monitor (Brünger, 1993).

\subsection{Molecular dynamics and discrete Fourier transform simulations}

Theoretical calculations were carried out by Martin Werner and Prof. Dr. Ricardo Mata (Institute of Physical Chemistry, University of Göttingen, Germany) in order to evaluate the role of steric shielding. The protein chain A in the asymmetric unit of the CspLOX2 crystal structure was used for the simulations and a water box was inserted in $8 \AA$ distance to the periodic cell limit. The charge was neutralized with $11 \mathrm{Na}^{+}$ions. Subsequently, 2000 steps followed for the minimization of the hydrogen atoms and 3000 steps for the optimization of all atoms. The Amber ff10 force field was used throughout. The parameters for the substrate radical were chosen according to the ones from Furse et al., 2006. After a heat-up phase of 50 ps to $300 \mathrm{~K}$ and an equilibration phase of 50 ps at this temperature, the production phase of the simulation followed.

For the determination of activation barriers, oxygen was narrowed in $0.1 \AA$ steps to the model system (2,5-heptadienyl radical) in relaxed surface scans using B3LYP-D3/def2-TZVP. The structure was optimized after each step, while leaving the oxygen-carbon distance and both dihedral angles around the middle position unchanged. One angle was always kept at $180^{\circ}$, the other one was varied from $150^{\circ}$ to $210^{\circ}$. The potentials were calculated for each $10^{\circ}$ step. 


\section{Results}

As LOX products can have different and even opposing regulatory functions in mammals and plants depending on the enzyme specificity, a lot of effort has been made to investigate how the regio- and stereoselectivity of the oxygenation is controlled on a molecular level. Since human and plant enzymes are sometimes more difficult to express or show a reduced stability, soluble bacterial enzymes which are structurally very similar might be good models to address this research question. In previous studies, two functional LOXs, namely CspLOX1 and CspLOX2, were identified in the diazotrophic cyanobacterium Cyanothece PCC 8801. X-ray crystal structures of both enzymes have recently been solved and revealed some unusual features of the overall domain structure. In the first part of this section, these unusual structural characteristics and their putative functions will be analyzed in comparison to other LOXs with special focus on the $\mathrm{N}$-terminal domain. The second and major part of this section will focus on CspLOX2 and its unusual ability to form bis-allylic hydroperoxides. Although this reaction was also reported for LOXs with manganese as catalytic metal, CspLOX2 is up to now the only bis-allylic LOX with a solved crystal structure and therefore provides detailed information about the active site architecture. As knowledge about this unusual specificity may also help to understand how the regio- and stereospecificity is controlled by LOXs in general, different questions were addressed in this study, including: What is the functional difference between the manganese and iron cofactor in LOXs? What kind of mutations around the putative substrate channel have the greatest impact on enzyme specificity and activity? How may the substrate be bound in the active site and which models may explain the specificity of CspLOX2? The following sections will shed light on these aspects.

\subsection{Cyanothece sp. expresses two LOXs with conserved catalytic domains but unusual $\mathrm{N}$-terminal domains}

\subsubsection{Sequence similarity of CspLOX1 to other LOXs is very low}

As cyanobacteria are assumed to be precursors of chloroplasts according to the endosymbiotic theory (Mereschkowsky, 1905), cyanobacterial LOXs were supposed to be good models for plastidic LOXs. But unexpectedly, sequence analysis of CspLOX1 and CspLOX2 (GenBank accession numbers WP_012595715.1 and WP_012596348.1, respectively) revealed that both LOXs from Cyanothece sp. are only distantly related to plastidic LOXs from plants on a sequence level (Figure 15). While CspLOX2 phylogenetically groups with other bacterial LOXs which share a common ancestor with animal LOXs, CspLOX1 was even placed as outgroup, indicating that it is not closely related to any of the here selected LOXs. The low sequence identity of less than $12 \%$ with other characterized LOXs was probably the reason why CspLOX1 was initially not identified as LOX in the genome of Cyanothece sp. by blast search, but only CspLOX2. 


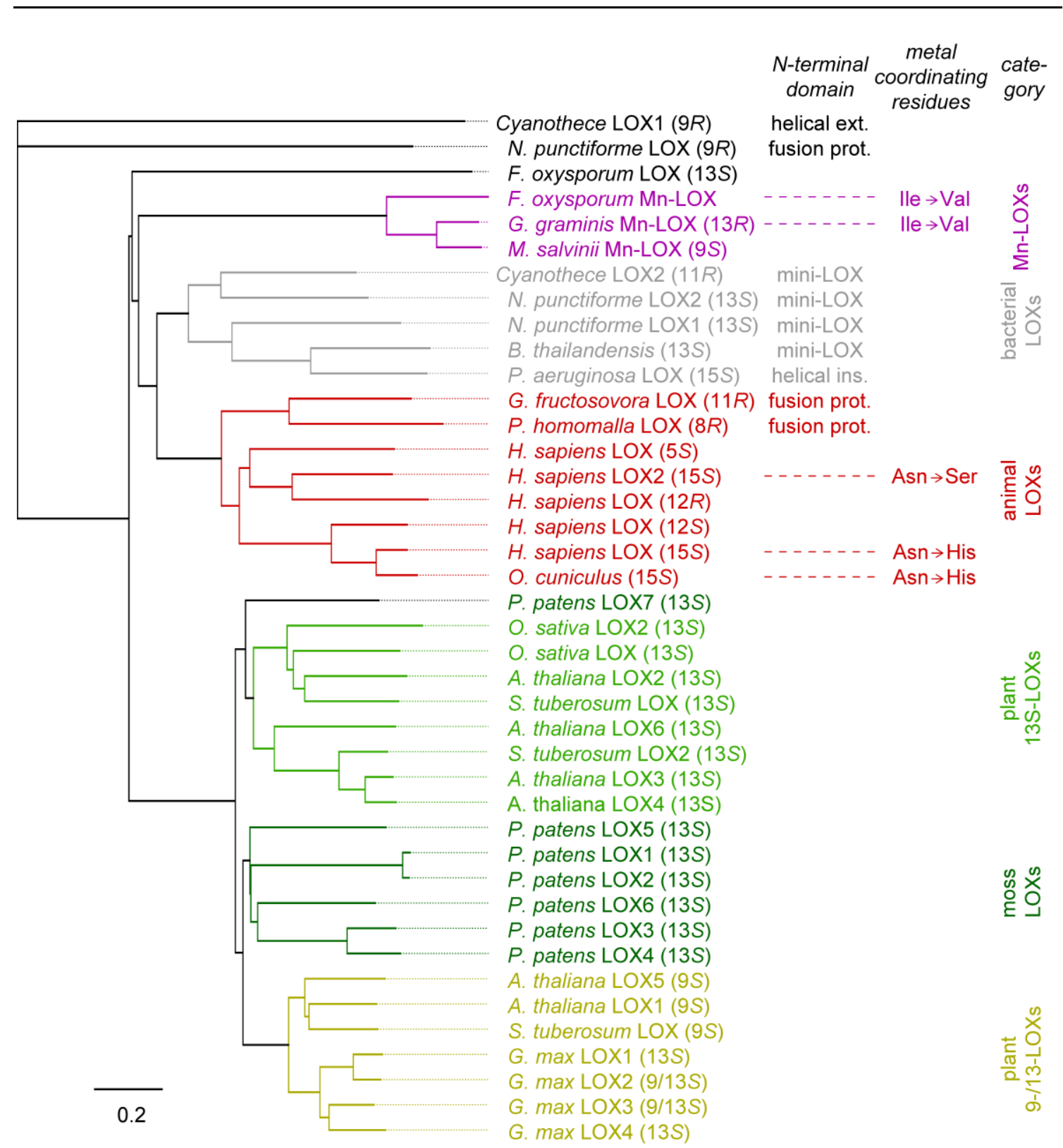

Figure 15. Phylogenetic tree of LOXs from different kingdoms of life. The organisms are indicated as well as their major specificities, variations of the N-terminal domain (ext., extension; prot., protein; ins., insertion) and variations of the metal coordinating residues (term., terminal). The tree was constructed with the program Geneious using the ClustalW algorithm. The proteins mentioned in the tree refer to the corresponding accession numbers in the GenBank: A. thaliana LOX1 (9S) (NP_175900.1), A. thaliana LOX2 (13S) (NP_566875.1), A. thaliana LOX3 (13S) (NP_564021.1), A. thaliana LOX4 (13S) (NP_177396.1), A. thaliana LOX5 (9S) (NP_188879.2), A. thaliana LOX6 (13S) (NP_176923.1), B. thailandensis (13S) (ABC36974), Cyanothece sp. LOX1 (9R) (WP_012595715.1), Cyanothece sp. LOX2 (11R) (WP_012596348.1), F. oxysporum LOX (13S) (EXK38530), F. oxysporum Mn-LOX (FOXB_09004), G. fructosovora LOX (11R) (AAY98506.1), G. graminis Mn-LOX (13R) (AAK81882.1), G. max LOX1 (13S) (NP_001236153.1), G. max LOX2 (9/13S) (NP_001237685.1), G. max LOX3 (9/13S) (CAA30016), G. max LOX4 (13S) (BAA03101), H. sapiens LOX2 (15S) (AAB61706.1), H. sapiens LOX (5S) (NP_000689.1), H. sapiens LOX (12R) (NP_001130.1), H. sapiens LOX (12S) (NP_000688.2), H. sapiens LOX (15S) (NP_001131.3), M. salvinii Mn-LOX (9S) (CAD61974.1), N. punctiforme LOX (9R) (WP_010994078.1), N. punctiforme LOX1 (13S) (WP_012412744.1), N. punctiforme LOX2 (13S) (WP_012407347.1), O.cuniculus (15S) (NP_001139620.1), O. sativa LOX2 (13S) (NP_001067011.1), O. sativa LOX (13S) (EEC72668), P. aeruginosa LOX (15S) (WP_023436288.1), P. homomalla LOX (8R) (AAC47743), P. patens LOX1 (13S) (XP_001784705.1), P. patens LOX2 (13S) (ABF66648), P. patens LOX3 (13S) (XP_001785004.1), P. patens LOX4 (13S) (ABF66650.1), P. patens LOX5 (13S) (ABF66651.1), P. patens LOX6 (13S) (ABF66652.1), P. patens LOX7 (13S) (ABF66653.1), S. tuberosum LOX (9S) (NP_001275357.1), S. tuberosum LOX (13S) (NP_001274843.1), S. tuberosum LOX2 (13S) (NP_001275115.1). 
More intense search, however, also identified CspLOX1 as putative LOX which was then characterized as functional enzyme with linoleate $9 R$-LOX activity by Dr. Alexandra Andreou (Newie et al., 2015).

\subsubsection{The catalytic domains of CspLOX1 and CspLOX2 resemble other LOXs}

CspLOX1 has recently been crystallized by Dr. Andreou (Newie et al., 2015), while CspLOX2 crystals were obtained in own work during previous studies. Both structures were solved by Dr. Neumann and refined to $2.8 \AA$ (CspLOX1) and $1.8 \AA$ (CspLOX2). These two models allowed a structural characterization and comparison with other LOX enzymes.

Even though the sequence similarity is rather low, both LOXs from Cyanothece sp. PCC8801 share the conserved LOX fold of the catalytic domain that is common to all LOX structures published so far (Figure 16, in gray). All long helices of the cylinder shaped domain are found at similar positions as in other LOXs except for the first helix of the catalytic domain which structurally corresponds to an extended loop region in CspLOX1 (Figure 18 and Figure 21B). Some peripheral parts of the catalytic domain have a slightly higher structural variability as well. A region that is for example comprised of six $\beta$-strands in CspLOX1 consists of short helices in other LOXs (Figure 16, dashed blue circle).
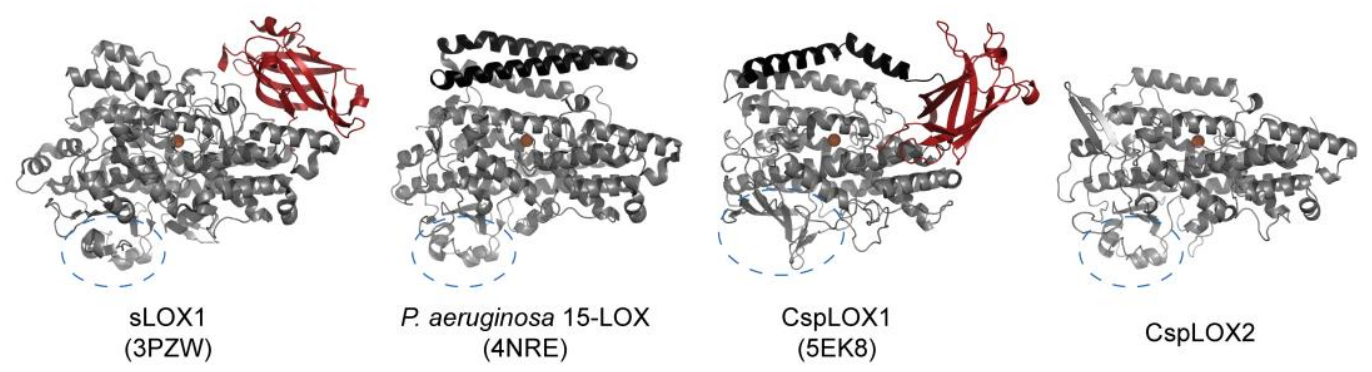

Figure 16. Comparison of the domain structure of different LOXs. The $\alpha$-helical catalytic domain which is present in all LOXs is shown in grey, the $\beta$-barrel in red and the long helical insertion/extension in black. PDB codes are given in brackets. The dashed blue circle highlights a region that corresponds to six $\beta$-strands in CspLOX1 and two short helices in other LOXs.

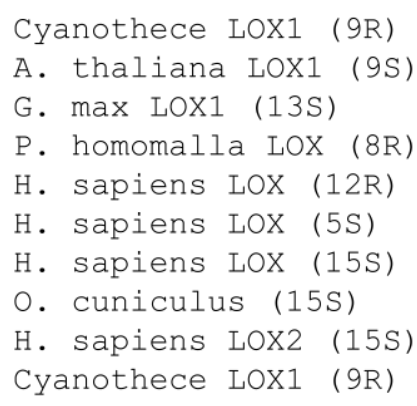

\begin{tabular}{|c|c|c|}
\hline 262 & $449 \quad 453$ & \\
\hline HVQ & - LWHTWVNDS & - \\
\hline & - ALHAAVNFG & \\
\hline & - $\mathrm{ALH}$ & \\
\hline T & $-\mathrm{CQH}$ & \\
\hline & - AK & \\
\hline & $-\mathrm{AQH}$ & \\
\hline M & - GQH & \\
\hline & - GQ & \\
\hline & - $\mathrm{AKH}$ & $-S T$ \\
\hline & - LWHTWVNDS & \\
\hline & & \\
\hline
\end{tabular}

Figure 17. Alignment of the metal coordinating residues of different LOXs. The residues involved in the coordination of the catalytic iron are highlighted in blue. Numbers indicate the positions of the respective residues in Cyanothece sp. LOX1 (CspLOX1) and Cyanothece sp. LOX2 (CspLOX2). GenBank accession numbers are as indicated for Figure 1. A larger alignment including more sequences is shown in Supplemental Figure 4. 
The catalytic iron of both Cyanothece sp. LOXs is coordinated by three histidines, one asparagine and the carboxy group of the $\mathrm{C}$-terminal isoleucine. These metal coordinating residues are highly conserved in most characterized LOXs from bacteria to plants (Figure 17), while in some mammalian LOXs, a fourth histidine or a serine occupies the position of the asparagine (Supplemental Figure 4). When LOXs from different organisms are superimposed, the rootmean-square deviations ( $\mathrm{rmsd}$ ) of the atomic positions of the $\mathrm{C} \alpha$ atoms can be determined. The low sequence similarity of CspLOX1 with other enzymes of this family is also reflected in a relatively low structural similarity to soybean LOX1, human 15S-LOX and coral 8R-LOX as indicated by large rmsd values of about $2.5 \AA$ for 350-450 C $\alpha$-atoms (Table 6). CspLOX2, on the contrary, is more similar to the same enzymes with rmsd values of less than $2 \AA$ for $450-550$ C $\alpha$ atoms.

Table 6. Structural comparison of CspLOX1 and CspLOX2 with other LOXs from soybean, human and the coral $P$. homomalla. Shown are the rmsd values in $\AA$ with the number of aligned $C \alpha$-atoms of the amino acids in brackets. These values were obtained by secondary structure matching in Coot. The second value given in percentage shows the sequence identity of the two compared LOXs. Primary sequences were aligned with the ClustalW algorithm using Geneious.

\begin{tabular}{|c|c|c|c|c|c|}
\hline & CspLOX1 & CspLOX2 & Soybean LOX1 & $\begin{array}{l}\text { Human } \\
\text { 15S-LOX2 }\end{array}$ & $\begin{array}{c}\text { Coral } \\
8 R \text {-LOX }\end{array}$ \\
\hline CspLOX1 & & $\begin{array}{c}1.91 \AA \text { ( }(368) / \\
9.46 \%\end{array}$ & $\begin{array}{c}2.60 \AA(392) / \\
10.81 \%\end{array}$ & $\begin{array}{c}2.53 \AA(415) / \\
11.6 \%\end{array}$ & $\begin{array}{c}2.50 \AA(368) / \\
9.17 \%\end{array}$ \\
\hline CspLOX2 & $\begin{array}{c}1.91 \AA ̊(368) / \\
9.46 \%\end{array}$ & & $\begin{array}{c}1.81 \AA \text { (469)/ } \\
17.32 \%\end{array}$ & $\begin{array}{c}1.68 \AA(499) / \\
19.79 \%\end{array}$ & $\begin{array}{c}1.59 \AA(514) / \\
22.92 \%\end{array}$ \\
\hline $\begin{array}{c}\text { Soybean } \\
\text { LOX1 }\end{array}$ & $\begin{array}{c}2.60 \AA(392) / \\
10.81 \%\end{array}$ & $\begin{array}{c}1.81 \AA ̊(469) / \\
17.32 \%\end{array}$ & & $\begin{array}{c}2.11 \AA ̊(574) / \\
17.09 \%\end{array}$ & $\begin{array}{c}1.91 \AA ̊(558) / \\
16.47 \%\end{array}$ \\
\hline $\begin{array}{c}\text { Human } \\
15 S \text {-LOX2 }\end{array}$ & $\begin{array}{c}2.53 \AA \text { (415)/ } \\
11.6 \%\end{array}$ & $\begin{array}{c}1.68 \AA \text { ( } 499) / \\
19.79 \%\end{array}$ & $\begin{array}{c}2.11 \AA(574) / \\
17.09 \%\end{array}$ & & $\begin{array}{c}1.37 \AA(607) / \\
28.57 \%\end{array}$ \\
\hline $\begin{array}{c}\text { Coral } \\
8 R \text {-LOX }\end{array}$ & $\begin{array}{c}2.50 \AA(368) / \\
9.17 \%\end{array}$ & $\begin{array}{c}1.59 \AA(514) / \\
22.92 \%\end{array}$ & $\begin{array}{c}1.91 \AA(558) / \\
16.47 \%\end{array}$ & $\begin{array}{c}1.37 \AA(607) / \\
28.57 \%\end{array}$ & \\
\hline
\end{tabular}

\subsubsection{The N-terminal domains of LOXs from Cyanothece sp. PCC8801 exhibit unusual features}

The most interesting structural difference of CspLOX1 and CspLOX2 compared to other LOXs is found within the N-terminal domain. Classical LOXs like soybean 13S-LOX1 or human 15S-LOX2 consist of two domains, with a $\beta$-barrel domain at the $\mathrm{N}$-terminus and the $\alpha$-helical catalytic domain at the C-terminus. CspLOX2, on the contrary, lacks the $\beta$-barrel domain completely and is therefore a so-called mini-LOX (Andreou et al., 2010). Some other bacterial LOXs lack this domain as well, like the ones from N. puntiforme (Koeduka et al., 2007) and B. thailandensis (An et al., 2015). Others exist as fusion proteins with a catalase-like domain. Removal of the catalaselike domain also resulted in active mini-LOX enzymes (Lang et al., 2008; Zhang et al., 2012; Zheng et al., 2008). Some bacterial LOXs have a helical insertion instead of the $\beta$-barrel domain like the P. aeruginosa 15S-LOX (Garreta et al., 2013) (Figure 16). CspLOX1 is special regarding the 
architecture of the N-terminal domain. It represents the first LOX that contains both, a $\beta$-barrel domain and additionally an $\alpha$-helical extension at the $\mathrm{N}$-terminus (Figure 18).

Comparison of this domain with those of other LOXs revealed that the topology of the antiparallel $\beta$-strands is in general very similar to those of other LOXs. Only one pair of $\beta$-strands is considerably elongated in CspLOX1 and points away from the core of the $\beta$-sandwich (Figure $18 \mathrm{C})$. Due to these elongated strands, both layers of the sandwich form a cleft that interacts with the catalytic domain. In addition to the extended antiparallel $\beta$-strands, the orientation of this domain relative to the cylinder shaped catalytic domain is different in the CspLOX1 crystal structure compared to other LOXs. While both domains are arranged almost parallel in human 15S-LOX2 or soybean 13S-LOX1 (Figure 18A and B), the barrel is rotated by more than $90^{\circ}$ in CspLOX1 (Figure 18C). Some parts of the $\beta$-barrel domain interact with the catalytic domain and structurally replace the helix $\alpha 1$ found in other LOXs (Figure 19A).

A

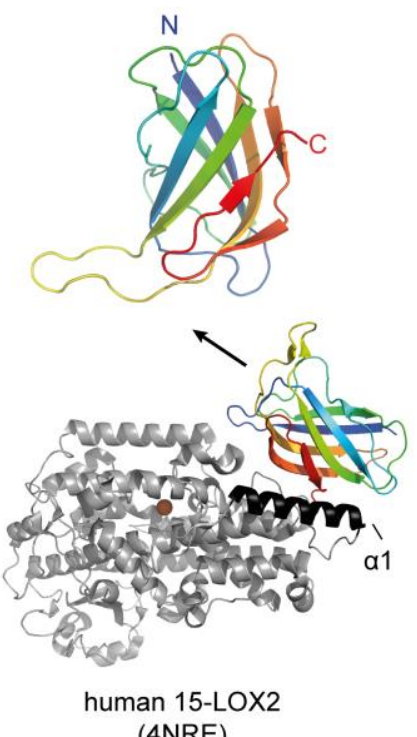

B

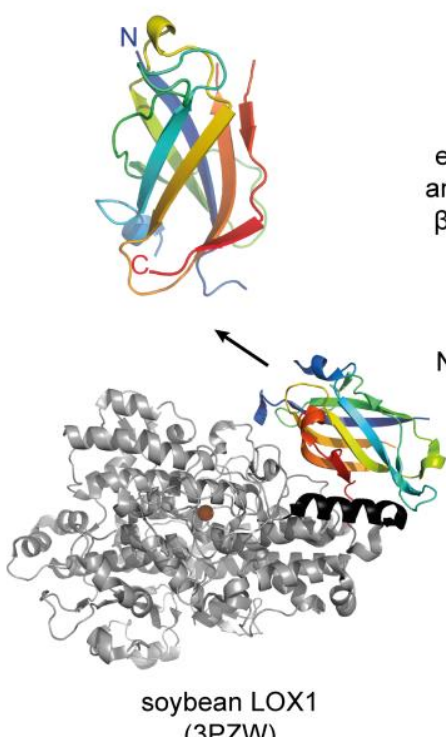

c

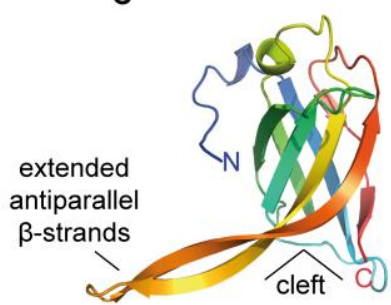

$\mathrm{N}$-terminal a-helices

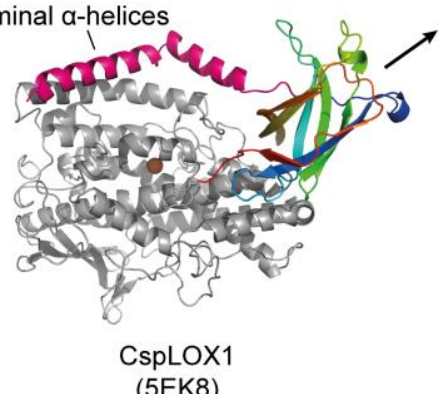

Figure 18. Comparison of the $\beta$-barrel domain of CspLOX1 with those of other LOXs. The overall arrangement of this domain is similar in different LOXs ( $\mathrm{A}$ and $\mathrm{B}$, rainbow colored from blue at the $\mathrm{N}$-terminus to red at the $\mathrm{C}$-terminus). Only two antiparallel $\beta$-strands are remarkably longer in CspLOX1 (C). Moreover, the first helix of the catalytic domain (black $\alpha 1$ ) is corresponding to an extended linker in CspLOX1. The orientation of the $\beta$-barrel domain (indicated by an arrow) relative to the catalytic domain is different in CspLOX1 compared to other LOXs. The catalytic iron is shown as orange sphere and the $\mathrm{N}$-terminal $\alpha$-helical extension of CspLOX1 is shown in pink. 

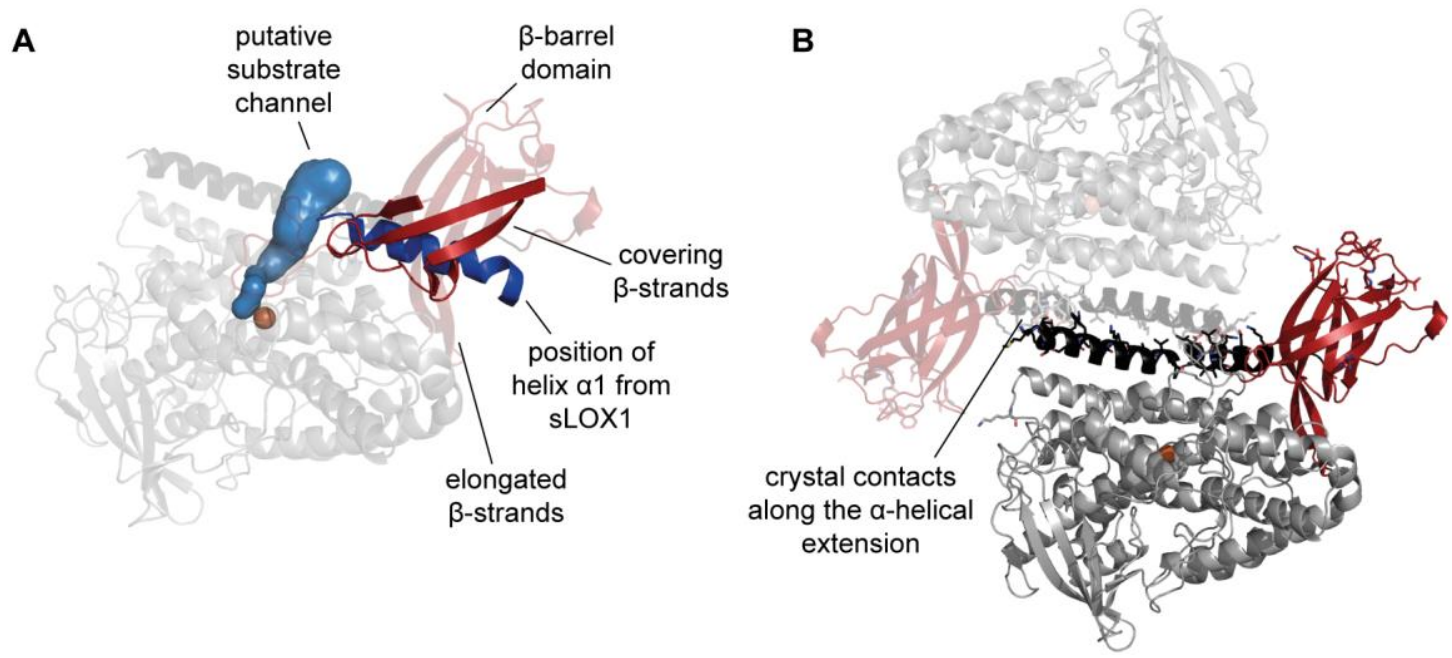

Figure 19. Interactions within CspLOX1 crystals. (A) Parts of the $\beta$-barrel domain structurally replace the covering function of the helix $\alpha 1$ (in blue) found in other LOXs. The part of the $\beta$-barrel in CspLOX1 replacing this covering helix is shown in red (opaque). (B) Crystal contacts between neighboring molecules in CspLOX1 crystals related by one 2fold axis. Amino acids involved in crystal contacts are shown as sticks and a neighboring molecule in the crystal lattice is shown as transparent cartoon. The iron is represented as orange sphere. Contacts were analyzed using the CCP4 suite (Winn et al., 2011).

The $\alpha$-helical extension of CspLOX1 is found $\mathrm{N}$-terminal of the $\beta$-barrel domain. This 40 amino acid extension consists of two amphipathic $\alpha$-helices that are connected by a short two amino acid linker. In the crystal structure, these $\mathrm{N}$-terminal helices are in contact with a long helix $\alpha 3$ of the catalytic domain comprising the residues 189 to 214 . Six hydrogen bonds and two salt bridges are formed between this helix and $\alpha$-helical extension and an area of $875 \AA^{2}$ is buried at the interface which corresponds to $23 \%$ of the total area of the helical extension (Figure 19B and Figure 20). The hydrophobic side of the helix is exposed to the surface and consequently needs to interact with a hydrophobic counterpart which is the $\alpha$-helical extension of a neighboring molecule. At this interface hydrophobic interactions and four hydrogen bonds are found and an area of $790 \AA^{2}$ is buried (Figure 19B). Interestingly, the entrance to the substrate binding channel is surrounded by mainly positively charged residues (Figure 20, blue), which could provide a favorable environment for the negatively charged carboxyl group of the fatty acid. 


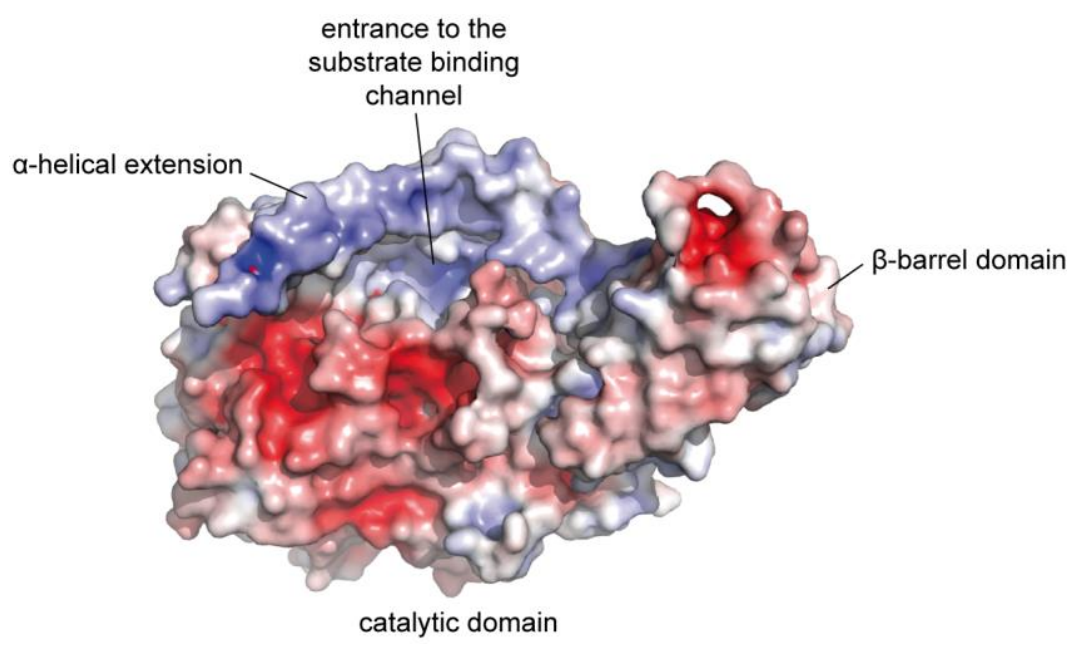

Figure 20. Surface potential map of CspLOX1. The map was generated with the APBS program (Baker et al., 2001). The electrostatic surface potential of CspLOX1, colored from red $(-7 \mathrm{kT} / \mathrm{e})$ to blue $(7 \mathrm{kT} / \mathrm{e})$, reveals basic patches around the entrance to the substrate binding channel.

\subsubsection{The $\alpha$-helical extension of CspLOX1 mediates binding to liposomes}

The $\beta$-barrel domain of LOXs has been discussed to be involved in membrane binding and may thus be important for substrate acquisition from the membrane. It was therefore interesting to test whether the ß-barrel of CspLOX1 can fulfill this function as well. Since it seemed possible that either the amphipathic $\alpha$-helical extension or the $\beta$-barrel domain or both mediate membrane binding of CspLOX1, truncated CspLOX1 versions were generated. The helical extension was cut off after Glu43 to produce a truncated version that contains the catalytic domain and the $\beta$-barrel domain. Another truncated version, which starts at Glu180, contains only the catalytic domain (Figure 21B and Figure 22). The protein versions were heterologously expressed in E. coli, purified and tested for their catalytic activity and their membrane binding ability. While the catalytic activity even increased after truncation of the $\alpha$-helical extension, the enzyme was inactive when additionally the $\beta$-barrel domain was removed (Figure 21A). The inactivity of the catalytic domain might be a result of the truncated linker region between the $\beta$ barrel and the catalytic domain. As the linker was cut rather in the middle of the loop, a large portion of it was removed by the truncation (Figure 21B). This linker, however, seems to cover parts of the active site. Similar loops over this area of the catalytic domain are also found in other LOXs such as sLOX1 or 15-LOX. Here, the loop is formed by amino acids of the catalytic domain found $\mathrm{C}$-terminal of the helix $\alpha 1$ (Figure 18). 

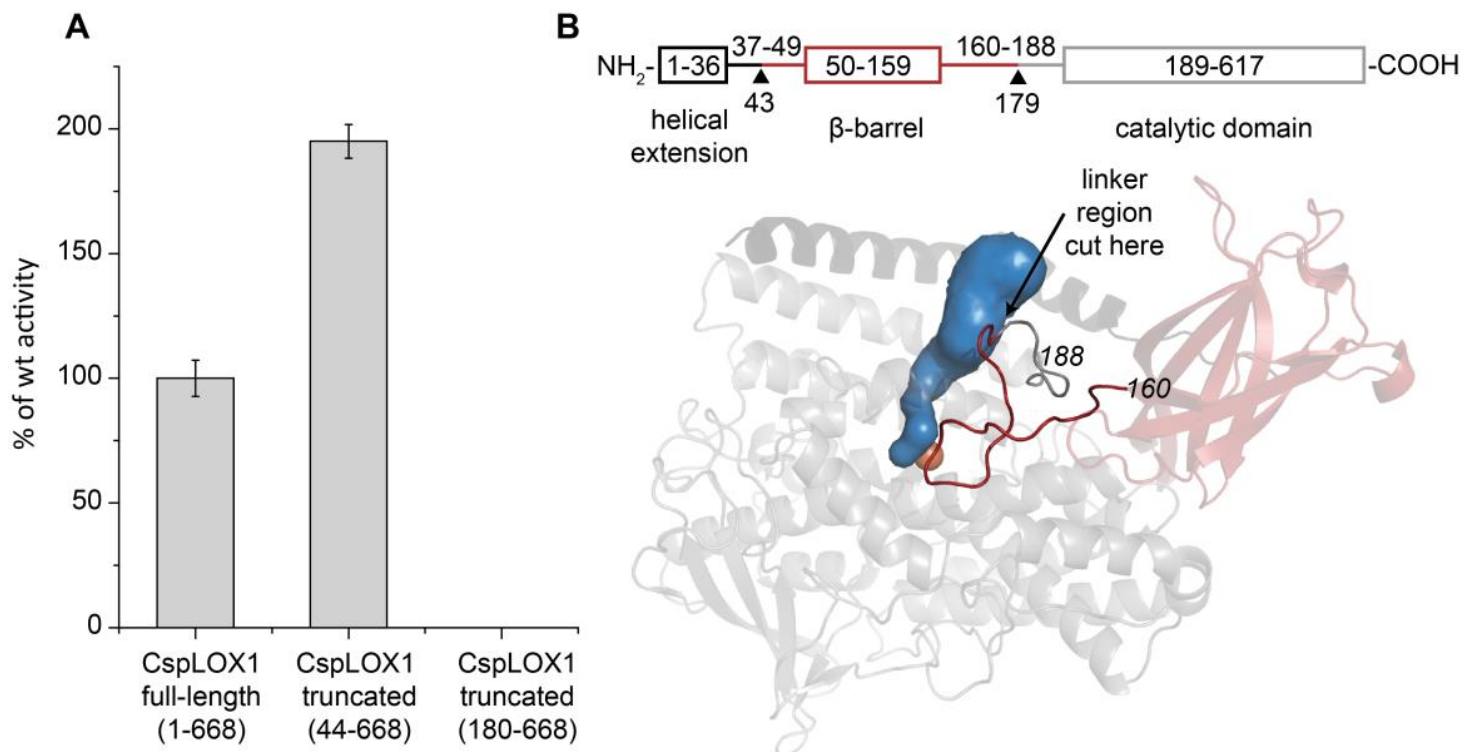

Figure 21. Analysis of the catalytic activities of the truncated CspLOX1 versions. (A) Relative catalytic activities compared to wt CspLOX1 (full-length) are shown, whereby the wt activity equals $100 \%$. Activities were measured with different substrate concentrations as increase at $234 \mathrm{~nm}$ with a spectrophotometer. Values were plotted using Origin to determine the kinetic constants according to the Michealis-Menten equation. (B) Scheme of the loop structure between the domains that were removed in the truncated versions. The boxes on top indicate different domains with linkers shown as lines in between. Amino acid positions are numbered. The structure below shows the second linker formed by amino acids 160-188 between the $\beta$-barrel domain and the catalytic domain with the site of truncation. As shown, a large part of the linker (red) was removed in CspLOX1 truncated (180-688). This part, however, forms a loop covering parts of the active site. The catalytic iron is shown as orange sphere, the putative substrate binding channel is shown in blue.

The membrane binding ability of the truncated versions of the enzyme was tested in a liposome binding assay. Liposomes consisting of the phospholipid 1,2-dipalmitoyl-sn-glycero-3phosphocholine resemble biological membranes as they consist of a lipid bilayer that surrounds an aqueous solution. In the assay, liposomes were incubated with the full-length or truncated versions of CspLOX1 (Figure 22). During a centrifugation step in a sucrose gradient, the liposomes float up with all proteins bound to them and will thus be found in the top fraction (T), while proteins with no affinity to liposomes will remain at the bottom (B). In a control experiment, only buffer instead of liposomes was incubated with the proteins (Figure 22, right lanes). SDS-PAGE analysis of individual fractions revealed that only the full-length version of CspLOX1 binds to the liposomes while binding is tremendously decreased in both truncated versions (Figure 22A vs. $\mathrm{B}$ and $\mathrm{C}$ ). These results suggest that the $\mathrm{N}$-terminal helical extension is necessary for an efficient membrane binding, while a clear contribution of the $\beta$-barrel domain could not be observed. CspLOX2, which lacks the N-terminal domains completely, has as expected no affinity for liposomes. 


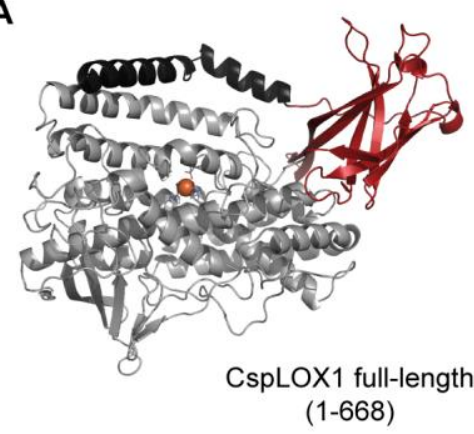

B

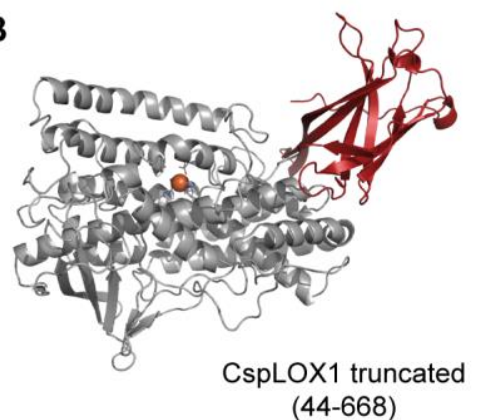

C

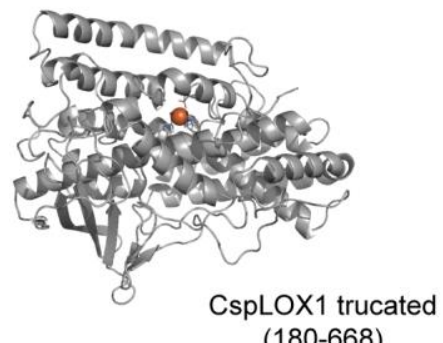

$(180-668)$

D

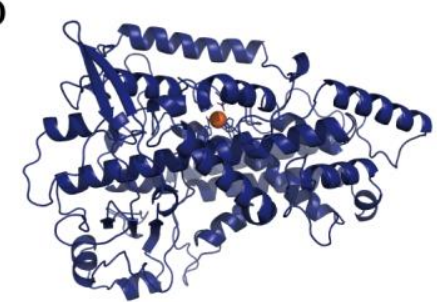

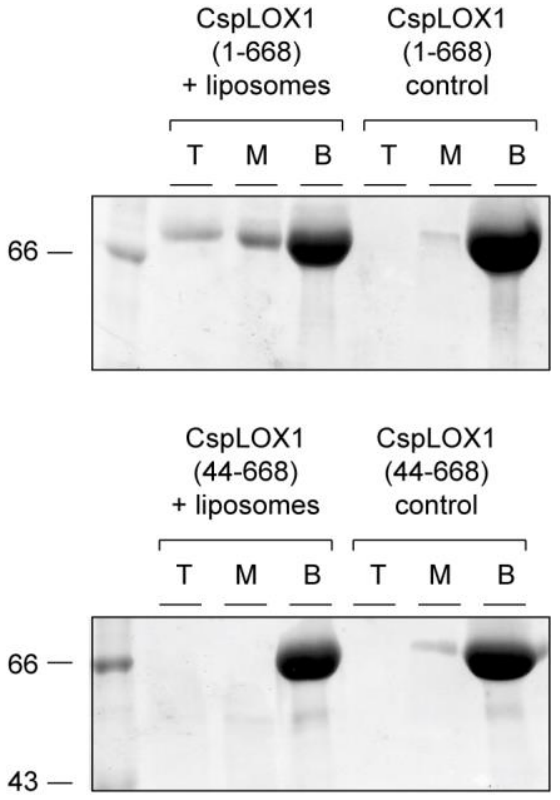
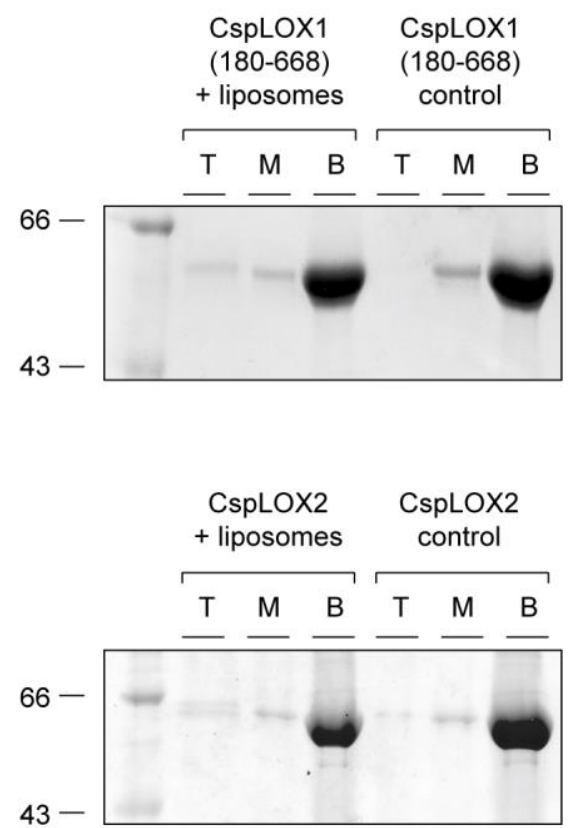

CspLOX2 full-length

Figure 22. Liposome binding assay of the truncated versions of CspLOX1. For this experiment, the N-terminal part of CspLOX1 was truncated after the $\mathrm{N}$-terminal $\alpha$-helix while retaining the $\mathrm{C} 2$-like domain (B) or after the $\mathrm{C} 2$-like domain, resulting in only the catalytic domain (C). These versions were compared to full-length CspLOX1 (A) and CspLOX2 (D). Liposomes were prepared with $10 \mathrm{mM}$ 1,2-dipalmitoyl-sn-glycero-3-phosphocholine in $100 \mathrm{mM}$ sodium phosphate buffer $\mathrm{pH} 7$ and incubated with $50 \mu \mathrm{g}$ of protein. During centrifugation in a sucrose gradient ( $250 \mu \mathrm{l} 30 \%$ sucrose, $200 \mu \mathrm{l} 25 \%$ sucrose and $50 \mu \mathrm{l} 0 \%$ sucrose), the proteins that have bound to liposomes will float up from the bottom of the tube (B) to the middle (M) and to the top fraction (T). Proteins in these three fractions were precipitated and analyzed by SDS-PAGE. As a negative control, proteins were incubated with only buffer instead of liposomes (right lanes). 


\subsection{CspLOX2 produces the instable 11-HPODE as an intermediate}

Differences between the LOXs from Cyanothece sp. and most other LOXs could not only be found in the structure, but also in the oxygenation specificity of the enzymes. While almost all characterized plant and animal LOXs catalyze the lipoxygenation with high S-specificity of the products, CspLOX1 and CspLOX2 form mainly products in an $R$-configuration: The main product of CspLOX1 is 9R-HPODE (Newie et al., 2015), while the main product of CspLOX2 is $11 R$-HPODE (Andreou et al., 2010). Although this is relatively rare, such $R$-specific LOXs have also been found in other organisms (Boutaud and Brash, 1999; Brodhun et al., 2013; Lang et al., 2008; Lõhelaid et al., 2008; Zhang et al., 2012). The most noticeable anomaly of the CspLOX2 specificity is, however, the ability to form bis-allylic hydroperoxides. No other iron-containing LOX was identified so far that produces bis-allylic products in significant amounts. The following section will focus on the formation and isomerization of this unusual product by CspLOX2.

\subsubsection{Optimized protocol required for reproducible 11-HPODE determination}

Although the formation of 11-HPODE has been reported for CspLOX2 (Andreou et al., 2010), it was initially difficult to isolate and analyze the rare oxylipin with high reproducibility. To overcome these problems and to establish a reliable and reproducible protocol for 11-HPODE analysis, the reaction, extraction and HPLC-analysis of 11-HPODE was optimized. During this optimization process, the following parameters were adjusted: reaction time, enzyme and substrate concentration, temperature of the reaction and extraction procedure.

The most challenging drawback of 11-HPODE is its instability. While 9- and 13-HPODE are rather stable after extraction, 11-HPODE was found be degraded over time, even when it was stored at $-80{ }^{\circ} \mathrm{C}$ in methanol or acetonitrile (Figure 23 ).
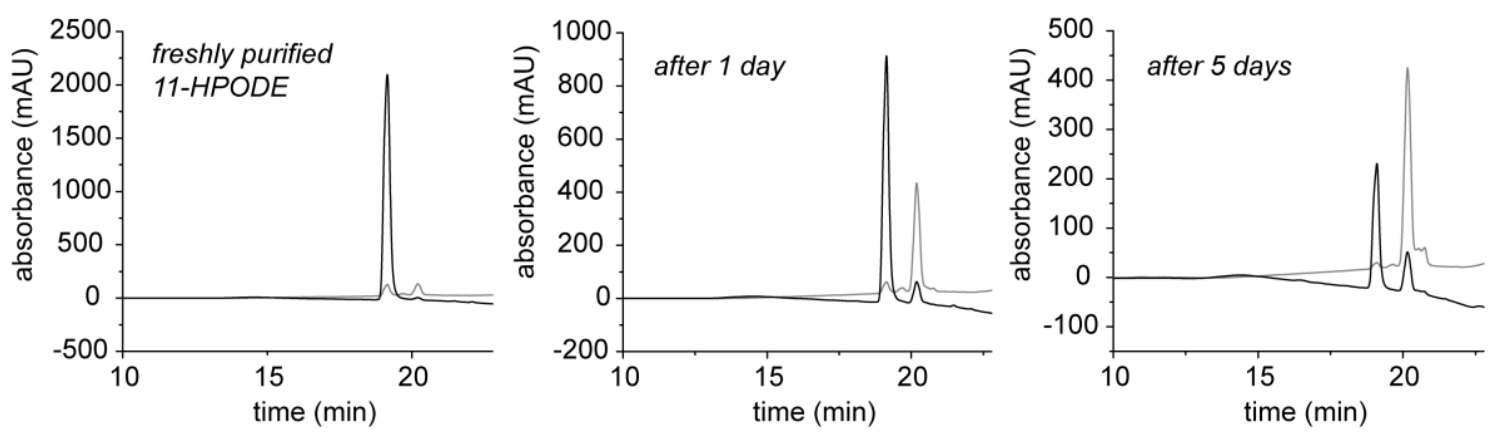

— at $202 \mathrm{~nm}(11-\mathrm{HPODE})$

- at $234 \mathrm{~nm}$ (9- and 13-HPODE)

Figure 23. Instability of 11-HPODE. 11-HPODE was enzymatically produced with CspLOX2 and purified by RP-HPLC. The product was dried in a stream of nitrogen, resuspended in methanol and stored at $-80{ }^{\circ} \mathrm{C}$. The remaining $11-$ HPODE was again analyzed by RP-HPLC directly after purification (left panel), after 1 day storage (middle panel) and after 5 day storage at $-80^{\circ} \mathrm{C}$ (right panel). 
Initial experiments suggested that the $\mathrm{pH}$ would control the oxygenation specificity, since no 11HPODE was detected when the reaction was performed at pH values lower than 9 (Figure 24A). Comparison of different conditions, however, revealed that acidification during extraction destroyed the bis-allylic product and was responsible for the lack of 11-HPODE with reaction buffers of lower $\mathrm{pH}$ values. Acidification of the reaction is generally used to improve the extraction of the protonated form of the carboxyl group which is more hydrophobic and hence less soluble in the aqueous phase. But since the extraction efficiency was not significantly affected without addition of acetic acid, acidification was from then on omitted. As a result, similar products were found in assays at $\mathrm{pH} 7$ and 9.5 (Figure 24B).

A

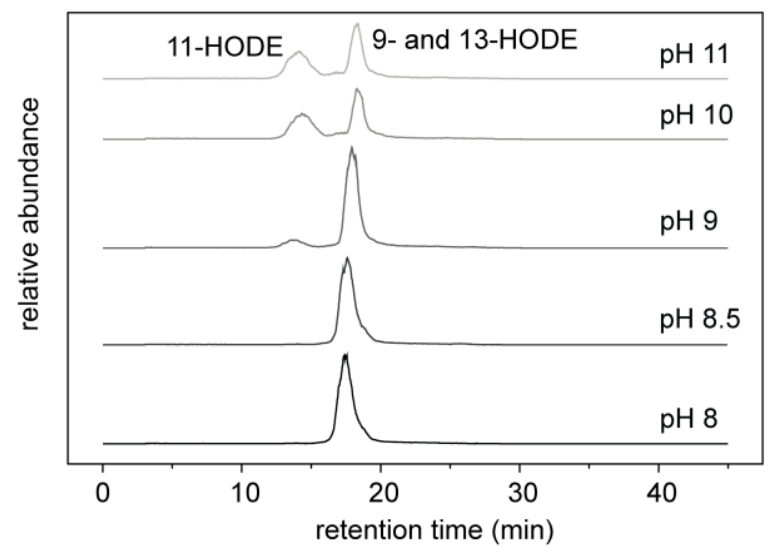

B

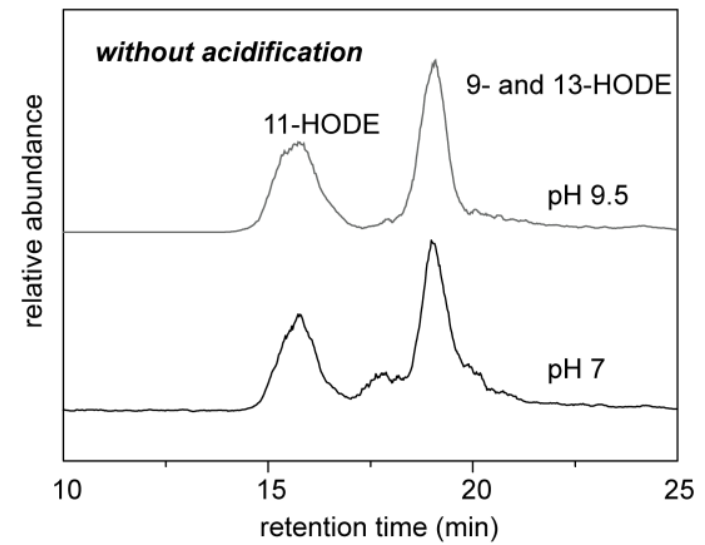

Figure 24. Acid lability of 11-HPODE. (A) $15 \mu \mathrm{g}$ of CspLOX2 were incubated with $100 \mu \mathrm{M}$ linoleic acid substrate at room temperature in $200 \mathrm{mM}$ sodium borate buffer ranging from $\mathrm{pH} 8$ to pH 11. Products were reduced with sodium borohydride and acidified with $100 \mu \mathrm{l}$ acetic acid. After extraction, products were analyzed by RP-HPLC-MS. (B) The experiment was repeated without acidification and products formed at $\mathrm{pH} 7$ and pH 9.5 were analyzed with the same method.

The optimization finally indicated that best results were obtained when the reaction was set up with $15 \mu \mathrm{g}$ CspLOX2 in $1 \mathrm{ml} 200 \mathrm{mM}$ sodium borate buffer $\mathrm{pH} 9$ and $100 \mu \mathrm{M}$ linoleic acid at $30^{\circ} \mathrm{C}$ for $6 \mathrm{~min}$. To stop the reaction, it was mixed with diethyether and placed on ice. After quick extraction, the products had to be analyzed on the same day by SP-HPLC. These established conditions were a prerequisite for the reliable analysis of enzyme variants with specifically exchanged amino acids.

\subsubsection{1-HPODE is only transiently formed}

Reactions of LOXs are usually monitored at $234 \mathrm{~nm}$ with a spectrophotometer to determine the catalytic activity, as the formation of conjugated double bonds can be observed at this wavelength. In comparison to other LOXs, two characteristics distinguish the reaction of CspLOX2 from most other LOXs. First of all, CspLOX2 exhibits a long kinetic lag phase that is observed as delay before the reaction starts. Secondly, the reaction comprises two linear phases with a first fast reaction rate and a second slower reaction rate (Figure 25A). On the contrary, LOXs from other organisms like sLOX1, CsLBLOX or FoxOX have remarkably shorter lag phases 
and only one linear reaction phase (Figure 25B). Only CspLOX1, the isozyme from Cyanothece sp., has a comparably long lag phase as CspLOX2 under the applied conditions.

Two reasons for the transition from the first to the second reaction phase seemed conceivable: Either the substrate is consumed at this point or the accumulating products could have an inhibitory effect on the reaction. To test these alternatives, the same amount of linoleic acid substrate that was present at the beginning of the reaction was added when the second reaction phase was reached. This resulted in a restart of a steep increase at $234 \mathrm{~nm}$ similar to the initial one, suggesting that the transition to the second phase is caused by a depletion of substrate (Figure 25C).

A

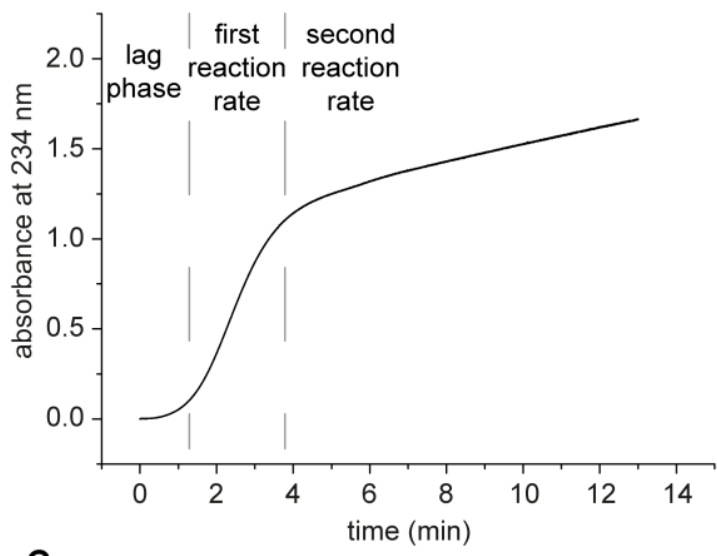

C

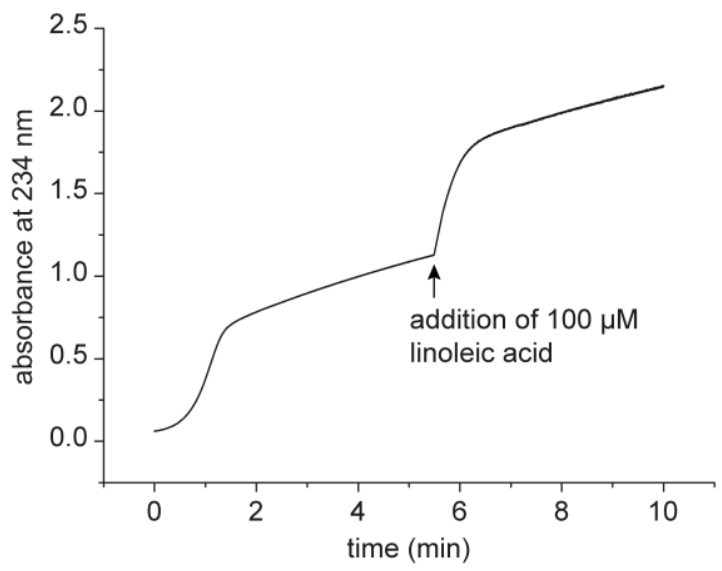

B

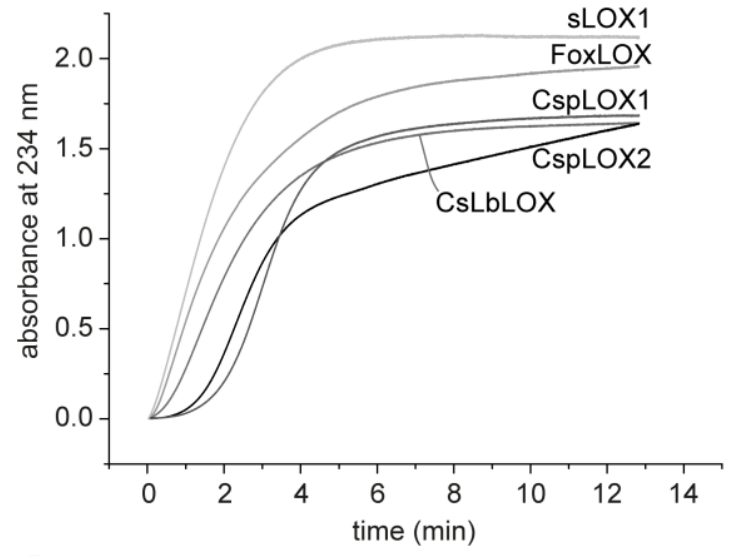

D

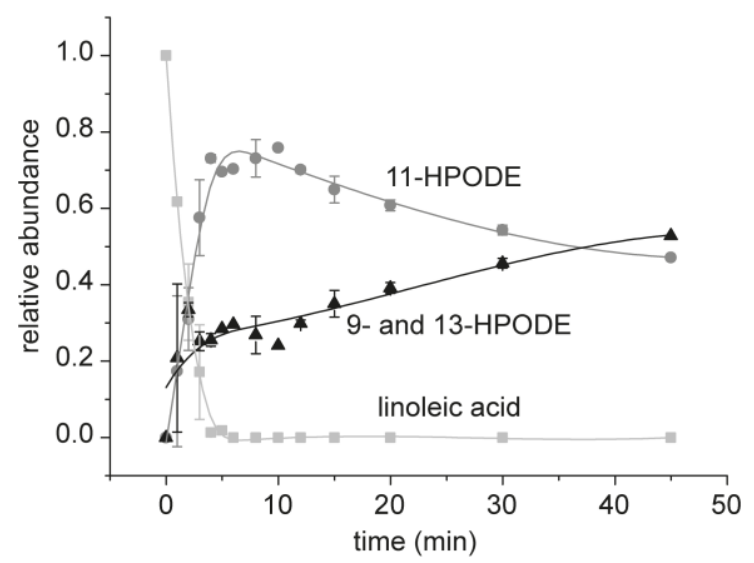

Figure 25. Individual phases of the CspLOX2 reaction. The LOX activity assay was performed in $200 \mathrm{mM}$ sodium borate buffer $\mathrm{pH} 9$ containing $100 \mu \mathrm{M}$ linoleic acid and started by adding enzyme. (A) The CspLOX2 reaction shows three distinct phases at $234 \mathrm{~nm}$. (B) These phases are not present in other LOX reactions. Different quantities of enzyme, purified by affinity chromatography, were used to achieve an increase in the same range. (C) Further addition of the same amount of substrate during the second phase restarts the fast first phase of the CspLOX2 reaction. (D) Relative amounts of the $\left[1-{ }^{14} \mathrm{C}\right]$-labeled linoleic acid substrate and hydroperoxy products were determined during the course of the CspLOX2 reaction. Samples were taken after different points of time. They were rapidly extracted and analyzed by radio RP-HPLC. The graph shows the progression of relative amounts. The values presented here are the mean values of 2 independent experiments. The data were interpolated by a B-spline function using Origin. 
It seemed likely that the unusual characteristics of the CspLOX2 progression curve at $234 \mathrm{~nm}$ are related to the formation of the bis-allylic product. On the one hand, the long lag phase could be a result of a predominant 11-HPODE formation at the beginning, since this product does almost not absorb at this wavelength (Figure 26). On the other hand, the second slower reaction could result from the isomerization of 11-HPODE to the conjugated hydroperoxides. In order to investigate these options, the ratio of bis-allylic and conjugated HPODEs was determined at different points of the reaction. The reaction was performed with $\left[1-{ }^{14} \mathrm{C}\right]$-labeled linoleic acid and samples were taken after different incubation times and the relative amounts of remaining linoleic acid substrate, formed 11-HPODE and conjugated HPODEs were analyzed by RP-HPLC. The resulting curves (Figure 25D) suggest that the substrate is depleted after $5 \mathrm{~min}$. This coincides with a peak of 11-HPODE which reaches its highest concentration after about 5 min and is then slowly declining again. The amount of conjugated 9- and 13-HPODE, which cannot be separated by this RP-HPLC method, resembles the progression curve observed at $234 \mathrm{~nm}$ (Figure 25A): they are first increasing rapidly and then slowly. These results strongly suggest that a mixture of HPODEs is formed in a fast reaction until the substrate is used up and that the formed 11-HPODE is subsequently isomerized to conjugated products explaining the second slower reaction rate observed at $234 \mathrm{~nm}$.
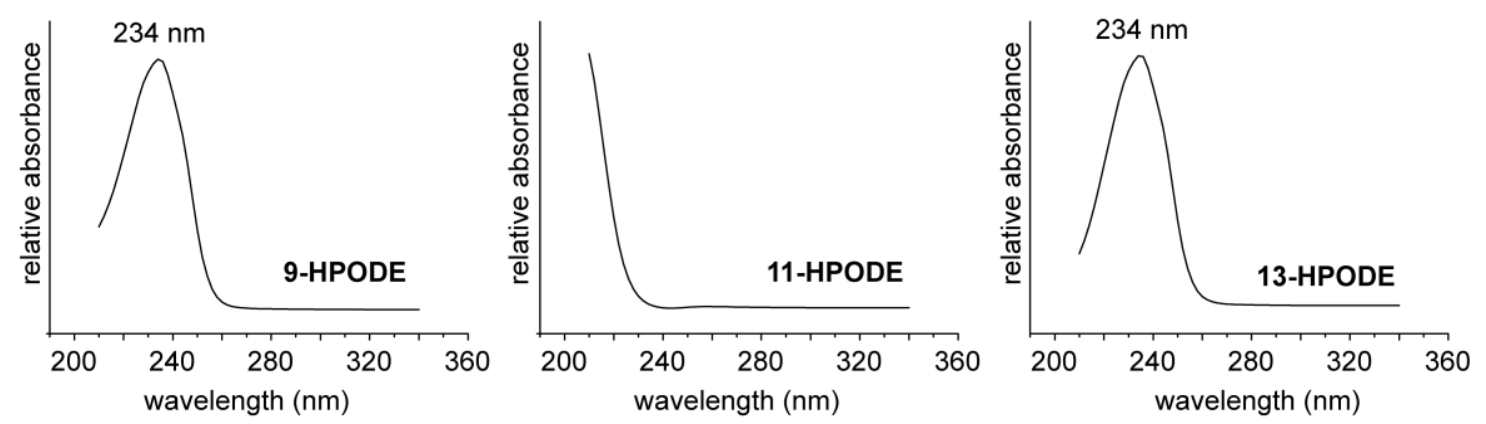

Figure 26. Absorption spectra of 9-, 11- and 13-HPODE. The absorption maxima of of 9- and 13-HPODE (234 nm) are indicated.

\subsubsection{Activation of CspLOX2 with HPODEs removes the lag phase}

As 11-HPODE does not absorb at $234 \mathrm{~nm}$ (Figure 26), the lag phase could also be attributed to an early formation of this bis-allylic product. For SLOX1 it was shown that the lag phase is a result of inactive enzyme in the as-isolated state, which contains $\mathrm{Fe}(\mathrm{II})$ in the active site. Upon addition of reaction product, i.e. HPODEs, the iron cofactor is oxidized to its active Fe(III) state to initiate the reaction (Schilstra et al., 1992; Schilstra et al., 1993). To test, whether inactive iron is also the reason for the long lag phase of CspLOX2, a small amount of reaction product was added to the assay. Indeed, addition of $1 \mu \mathrm{M}$ HPODE was enough to directly initiate the reaction without delay (Figure 27). These results suggest that the reason for the observed lag phase is the same as in other LOXs: the activation of the catalytic iron from $\mathrm{Fe}(\mathrm{II})$ to $\mathrm{Fe}(\mathrm{III})$ by hydroperoxides. Although the lag phase is longer compared to the reactions of other LOXs (Figure 25B), it is apparently not related to 11-HPODE formation. Therefore, similar results should be obtained if 
the consumption of oxygen is monitored using an oxygen electrode. With this method, the oxygen insertion at all possible positions is detected. As expected, the addition of HPODEs also shortened the lag phase of CspLOX2 here (Figure 27B). It should be noted that the progression curve observed with the oxygen electrode exhibits only one reaction phase. This is in line with the assumption that the second reaction rate observed at $234 \mathrm{~nm}$ accounts for the isomerization, since this would not consume further oxygen.

A

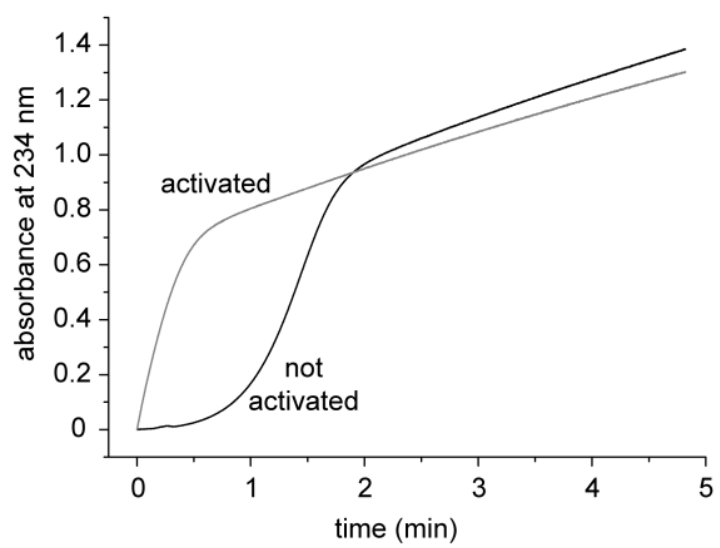

B

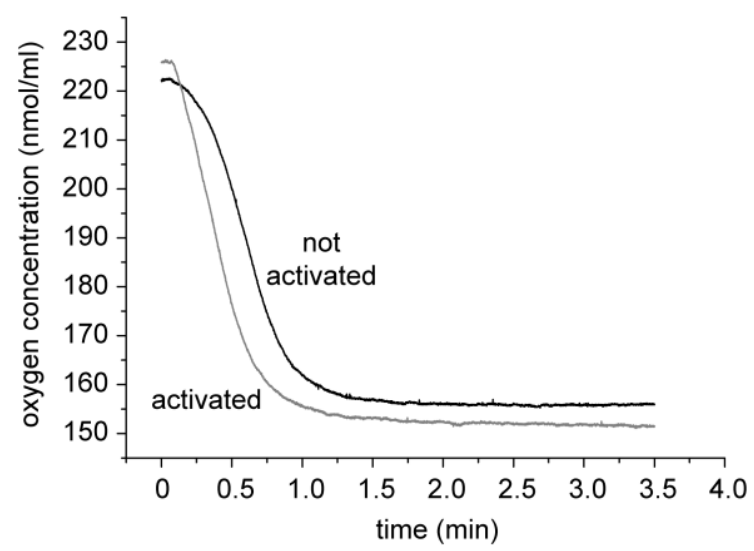

Figure 27. Activation of the CspLOX2 reaction by addition of HPODEs. (A) Freshly purified CspLOX2 was incubated with $100 \mu \mathrm{M}$ linoleic acid and the reaction was monitored at $234 \mathrm{~nm}$ (not activated). If $1 \mu \mathrm{M}$ reaction product, i.e. HPODEs, was added to the assay, the reaction starts directly without lag phase (activated). (B) The experiment was repeated using an oxygen electrode to monitor the decrease of oxygen. This resulted in the same effect.

\subsubsection{Kinetics of the isomerization reaction exclude model of reaction sequence}

Another interesting question was if the CspLOX2 reaction proceeds as a sequence (Figure 29A). In this case, all linoleic acid substrate would first be converted to the 11-HPODE intermediate which would then be isomerized to the conjugated HPODEs. Therefore, the kinetics of the isomerization reaction were determined and compared to the kinetics observed with linoleic acid. For the analysis of the isomerization, 11-HPODE was enzymatically produced with CspLOX2 and purified by RP-HPLC. The concentrations of produced 11-HPODE solution was determined by converting small aliquots completely with CspLOX2 and calculating the amount of produced conjugated HPODEs. Different concentrations of 11-HPODE were then converted with CspLOX2 and the increase in absorbance at $234 \mathrm{~nm}$ was monitored (Figure 28A). The results were fitted to the Michealis-Menten equation and a turnover number $k_{\text {cat }}$ of $0.58 \mathrm{~s}^{-1}$ and a Michaelis-Menten constant $K_{\mathrm{M}}$ of $4 \mu \mathrm{M}$ 11-HPODE were determined for the isomerization reaction. In comparison, the first fast reaction observed with linoleic acid proceeds at a 10-fold higher rate $\left(5.5 \mathrm{~s}^{-1}\right)$. The $k_{\text {cat }}$ of $0.50 \mathrm{~s}^{-1}$ determined for the second reaction rate, however, resembles the one of the isomerization reaction (Figure 28B). This is in line with the results shown in Figure 25D. It strongly suggests that the second reaction rate observed at $234 \mathrm{~nm}$ indeed represents the isomerization reaction. Furthermore, it can be concluded that the CspLOX2 reaction cannot proceed as a sequence in which all substrate is first converted to $11-\mathrm{HPODE}$, before it is isomerized. This model would be in conflict with the fast initial reaction rate. During this phase conjugated 
hydroperoxides are formed at a 10-fold higher rate than the $k_{\text {cat }}$ of the isomerization (Figure 29A vs. B).
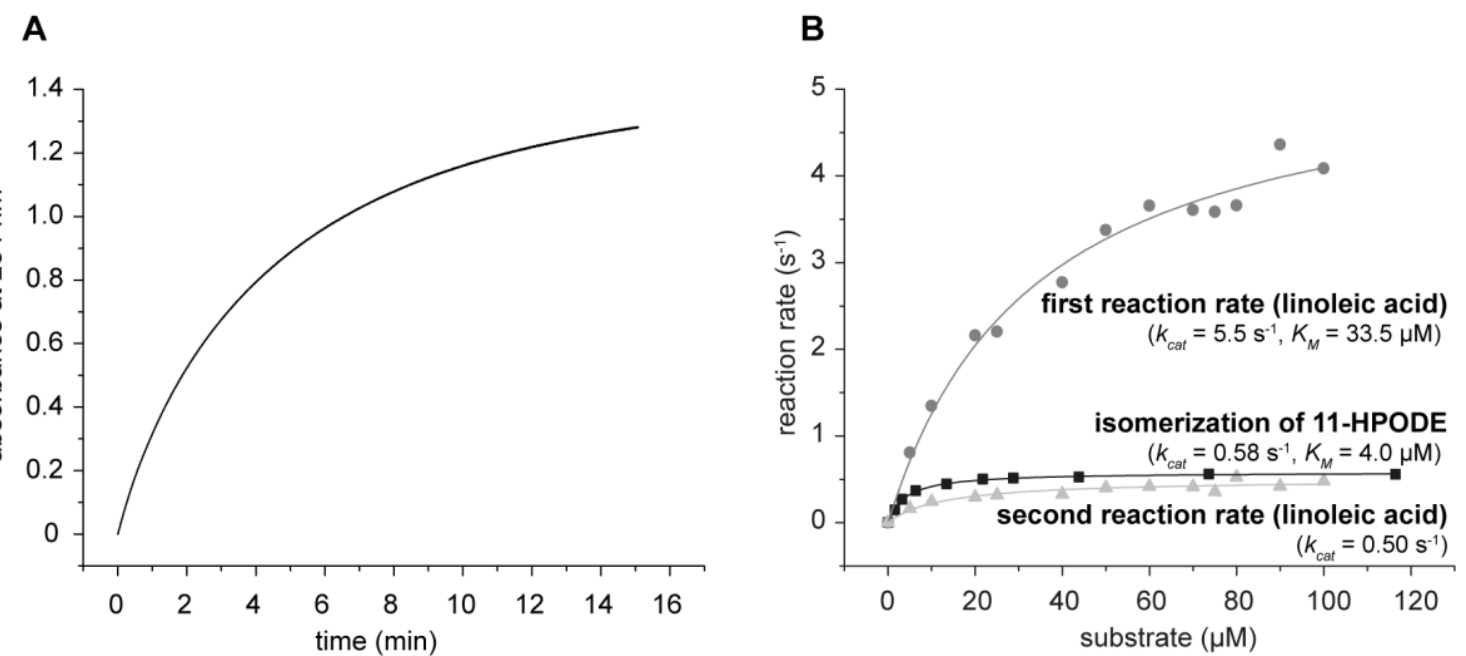

Figure 28. Enzyme kinetics of the isomerization reaction. 11R-HPODE was enzymatically produced with CspLOX2 and purified by RP-HPLC. (A) 11-HPODE was incubated with CspLOX2. The formation of conjugated double bonds was monitored at $234 \mathrm{~nm}$. The progression curve shows only one phase and the reaction starts immediately without lag phase. (B) Michaelis-Menten kinetics of the isomerization reaction in comparison to the first and second reaction rate of the CspLOX2 reaction with linoleic acid. Different amounts of linoleic acid and 11-HPODE were used and the activity determined from the increase at $234 \mathrm{~nm}$ was plotted against the substrate concentration.

A

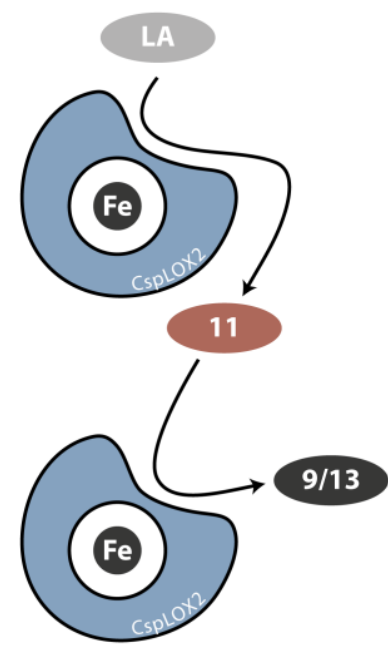

reaction sequence
B

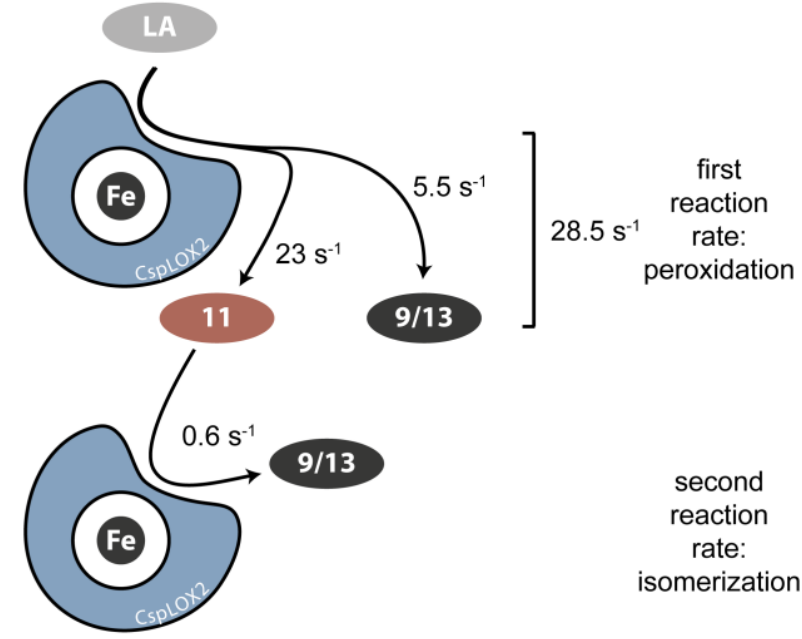

mixed HPODEs and isomerization

Figure 29. Model of the reaction steps catalyzed by CspLOX2. Two options seemed possible: (A) The reaction proceeds as a sequence. Here, linoleic acid (LA) would first be converted to 11-HPODE as an obligatory intermediate before it is isomerized to the conjugated 9- and 13-hydroperoxides. (B) During the peroxidation phase, a mixture of HPODEs is formed, and only the portion of 11-HPODE is subsequently isomerized to the conjugated products. Our results are clearly in favor of model B. 


\subsubsection{1-HPODE is the major product of the first reaction phase}

A possibility to separately analyze the 11-HPODE formation is to compare the reaction rates determined using the oxygen electrode with the reaction rates determined at $234 \mathrm{~nm}$ using a spectrophotometer. While the oxygen electrode detects the insertion of oxygen at all positions of the substrate, the spectrophotometer detects only the formation of conjugated HPODEs at $234 \mathrm{~nm}$. The rate difference of both methods should therefore account for the 11-HPODE formation. For CspLOX2, a $k_{\text {cat }}$ of $28.5 \mathrm{~s}^{-1}$ was determined with the oxygen electrode and a $k_{\text {cat }}$ of $5.5 \mathrm{~s}^{-1}$ at $234 \mathrm{~nm}$ with the spectrophotometer (Table 7). The rate of 11-HPODE formation should therefore be $23 \mathrm{~s}^{-1}$. Consequently, about $80 \%$ of the initial reaction rate account for the 11 HPODE formation and $20 \%$ for the formation of conjugated HPODEs. This is in line with the ratio determined with a full conversion of linoleic acid. The reaction was followed at $234 \mathrm{~nm}$ until the plateau was reached (Figure 30A). At this point all linoleic acid substrate will be ultimately converted to 9- and 13-HPODE. It thus marks the end point (100 \%) of the reaction. As shown in Figure $30 \mathrm{~A}$, the transition of the first to the second reaction phase occurs at about $20 \%$. This ratio is also supported by the product composition obtained with $\left[1-{ }^{14} \mathrm{C}\right]$-labeled linoleic acid at the end of the first reaction phase. Here, a ratio of 75:25 of 11-HPODE to conjugated HPODEs was determined (Figure 30B) which is in good agreement with both other experiments.

Table 7. Kinetics measured using the spectrophotometer and the oxygen electrode. The turnover numbers $k_{\text {cat }}$ determined from the oxygen decrease and increase in absorbance at $234 \mathrm{~nm}$ are compared. Using the oxygen electrode, the formation of 9-, 11- and 13-HPODE can be detected, while only the formation of 9- and 13-HPODE is detected at $234 \mathrm{~nm}$. The difference of both measurements should thus reflect the velocity of 11-HPODE formation.

\begin{tabular}{lll} 
& $k_{\text {cat }}\left(\mathrm{s}^{-1}\right)$ & relative rate (\%) \\
\hline oxygen electrode (all HPODEs) & $28.5 \pm 0.7$ & 100 \\
\hline absorption at 234 $\mathrm{nm}$ (only 9- and 13-HPODE) & $5.5 \pm 0.3$ & 19.3 \\
\hline difference (11-HPODE) & 23 & 80.7
\end{tabular}

A

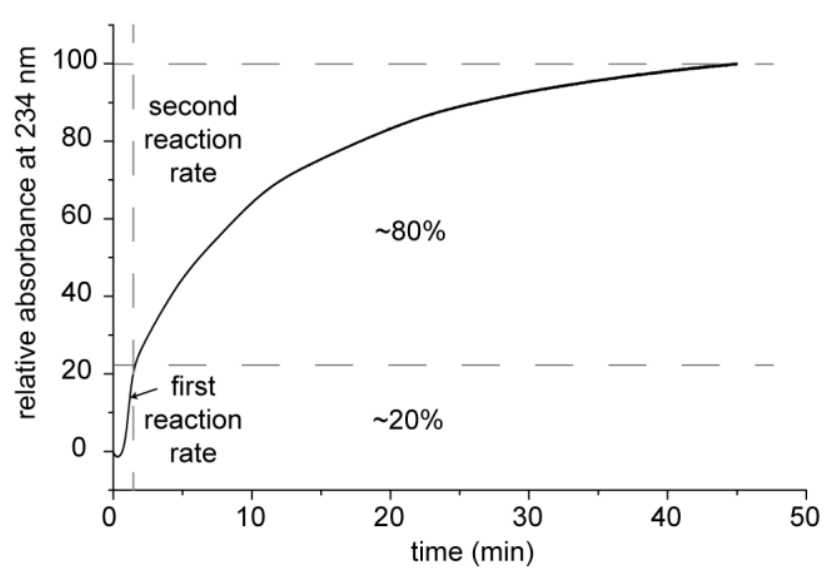

B

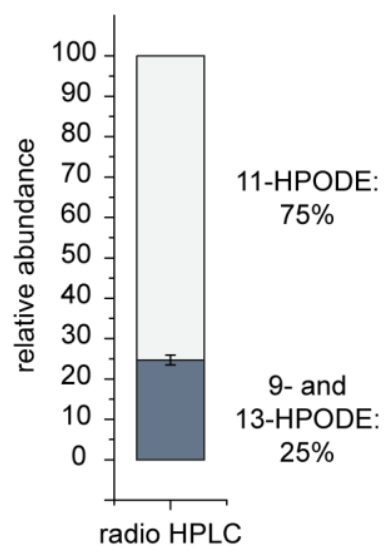

Figure 30. Ratio of 11-HPODE to conjugated products at the end of the first reaction phase. (A) CspLOX2 was incubated with $100 \mu \mathrm{M}$ linoleic acid and the reaction was monitored until almost no increase in absorbance at $234 \mathrm{~nm}$ was detected anymore. The end point of the reaction reflects almost a complete conversion to conjugated HPODEs from linoleic acid and was set to $100 \%$. (B) Products formed from $\left[1-{ }^{14} \mathrm{C}\right]$-labeled linoleic acid were analyzed after the first reaction phase with CspLOX2. Products were rapidly extracted after the first reaction phase and analyzed by RP-HPLC equipped with a radio-detector. The chart shows the mean values and standard deviation of three independent experiments. 


\subsubsection{Some other LOXs can also isomerize 11-HPODE to conjugated HPODEs}

Since 11-HPODE is a rather unstable product and intense optimization of the extraction process was required to obtain reliable results (Chapter 3.2.1), it might be possible that other ironcontaining LOXs can produce 11-HPODE under these optimized conditions as well. To test this, different LOXs from plants (sLOX1, CsLbLOX), cyanobacteria (CspLOX1) and fungi (FoxLOX) were tested for their ability to produce 11-HPODE from linoleic acid. But even under these optimized conditions none of the tested enzymes produced 11-HPODE (data not shown). Furthermore, it was tested whether these LOXs can isomerize 11-HPODE to the conjugated products. And indeed two of the four tested enzymes, FoxLOX and CspLOX1, were able to isomerize the purified 11-HPODE to conjugated products. This could be observed as increase in absorbance at $234 \mathrm{~nm}$ (Figure 31A). Further analysis of the products revealed that the isomerization products had the same positional specificity as the peroxidation products from linoleic acid. The 13-LOX FoxLOX (Brodhun et al., 2013) produced primarily 13-HPODE, while the 9-LOX CspLOX1 (Newie et al., 2015) produced almost exclusively 9-HPODE (Figure 31B). The products of the 11-HPODE isomerization catalyzed by CspLOX2 are also the same as observed after $2 \mathrm{~h}$ with linoleic acid.

A

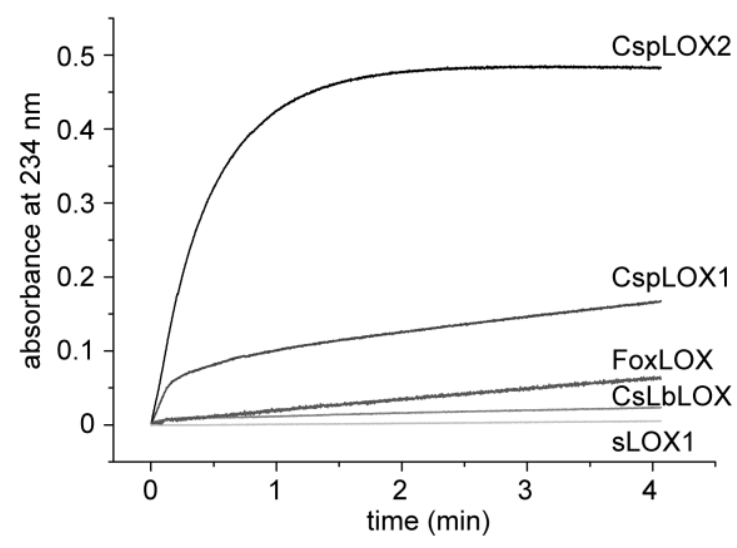

B

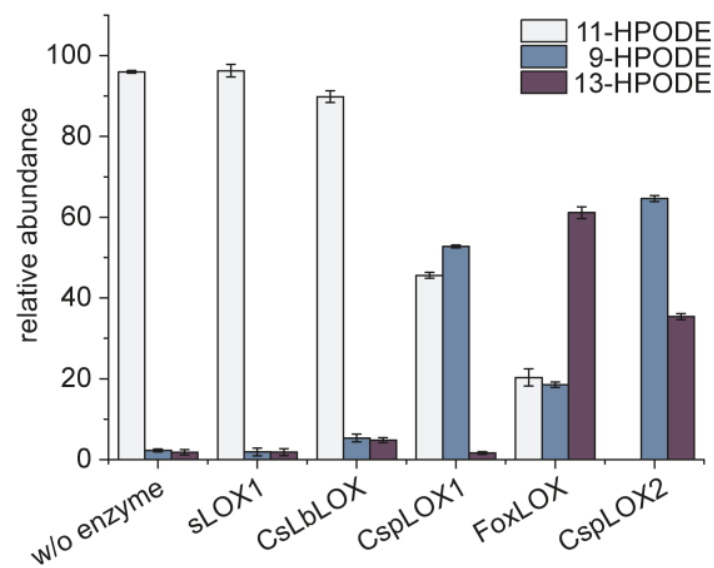

Figure 31. Isomerization of 11-HPODE with different LOXs. (A) 11-HPODE was produced with CspLOX2, purified by RPHPLC and incubated with $600 \mathrm{U}$ of different LOXs. Reactions with CspLOX2, CspLOX1 and FoxLOX resulted in a slight increase at $234 \mathrm{~nm}$, whereas CsLbLOX and sLOX1 showed virtually no increase. (B) The reaction products were extracted after $15 \mathrm{~min}$ and analyzed by SP-HPLC. The remaining 11-HPODE was quantified at $202 \mathrm{~nm}$ and 9- and 13HPODE at $234 \mathrm{~nm}$. The scaling factors determined with $\left[1-{ }^{14} \mathrm{C}\right]$-labeled compounds (Supplemental Figure 2) were included in the quantification. As a negative control, only buffer was incubated with 11-HPODE (w/o enzyme). 


\subsection{EPR and ICP-AES studies reveal that CspLOX2 is only active with iron}

Since most LOXs that produce bis-allylic hydroperoxides contain manganese instead of iron in the active site, it was interesting to study the role of the catalytic metal in the active site in more detail. For an initial characterization of the CspLOX2 metal cofactor, EPR spectra were recorded at $9.4 \mathrm{GHz}$ by Dr. Müge Kasanmascheff (EPR Spectroscopy Group, MPI for Biophysical Chemistry, Göttingen). The as-isolated state of the enzyme was compared with the activated state upon addition of HPODEs and during reaction with linoleic acid. As expected, the characteristic iron signal of iron-containing LOXs (Gaffney et al., 1993; Slappendel et al., 1981) could be identified (Figure 32A, upper part). In the as-isolated state, the signal was characteristic for Fe(II) which is EPR silent at this frequency, whereas a typical Fe(III) signal appeared in the activated state. Interestingly, the spectrum additionally revealed a sharp manganese six line signal that disappeared when HPODE or linoleic acid was added (Figure 32A, upper part). It thus seemed possible that also manganese can fulfill a catalytic function in CspLOX2 or is even responsible for the amount of 11-HPODE that is produced, as similar manganese signals have been reported for MnLOXs (Oliw et al., 2011; Su et al., 2000; Wennman et al., 2015).

\subsubsection{The iron cofactor of CspLOX2 can be substituted by manganese}

To assess whether iron or manganese or both metals are functional in the active site of CspLOX2, it was desirable to produce CspLOX2 variants that contain either manganese or iron in the active site. Initial trials to extract the catalytic iron with chelators like phenanthrolin were not successful. Another possibility to substitute the catalytic metal is to adjust the expression parameters by only including the desired metal in the medium, while excluding other transition metals. Finally, different CspLOX2 versions could be obtained by protein expression in minimal auto-induction medium that was supplemented with either manganese or iron. During the purification process, transition metals were excluded from buffers as far as possible. To elucidate whether the metal cofactor could indeed be exchanged, the purified proteins were subjected to ICP-AES. These measurements were carried out by Uta Nüsse-Hahne (Department of Plant Ecology and Ecosystems Research, University of Göttingen) and revealed that FeCspLOX2 contains 97 mol \% Fe and only 1 mol \% Mn, whereas Mn-CspLOX2 contains 62 mol \% $\mathrm{Mn}$ and only $2 \mathrm{~mol} \%$ Fe (Table 8).

Table 8. Metal content and catalytic activity of Mn- and Fe-CspLOX2. Different CspLOX2 versions were expressed in PA-5052 minimal medium containing either iron or manganese to produce Fe-CspLOX2 and Mn-CspLOX2, respectively, or in complex medium with a metal mix containing both manganese and iron (CspLOX2(mix)). The metal content of the CspLOX2 variants determined by ICP-AES is indicated as a molar percentage of metal per enzyme. The $k_{\text {cat }}$ was determined using the oxygen electrode.

\begin{tabular}{cccc} 
& Fe-CspLOX2 & Mn-CspLOX2 & $\begin{array}{c}\text { CspLOX2 } \\
(\operatorname{mix})\end{array}$ \\
\hline Fe occupancy $(\mathrm{mol} \%)$ & 96.6 & 2.1 & 58.4 \\
\hline Mn occupancy $(\mathrm{mol} \%)$ & 1.3 & 61.8 & 18.1 \\
\hline$k_{\text {cat }}\left(\mathrm{s}^{-1}\right)$ & $35.3 \pm 3.1$ & $1.9 \pm 0.6$ & $28.5 \pm 0.7$ \\
\hline
\end{tabular}


A

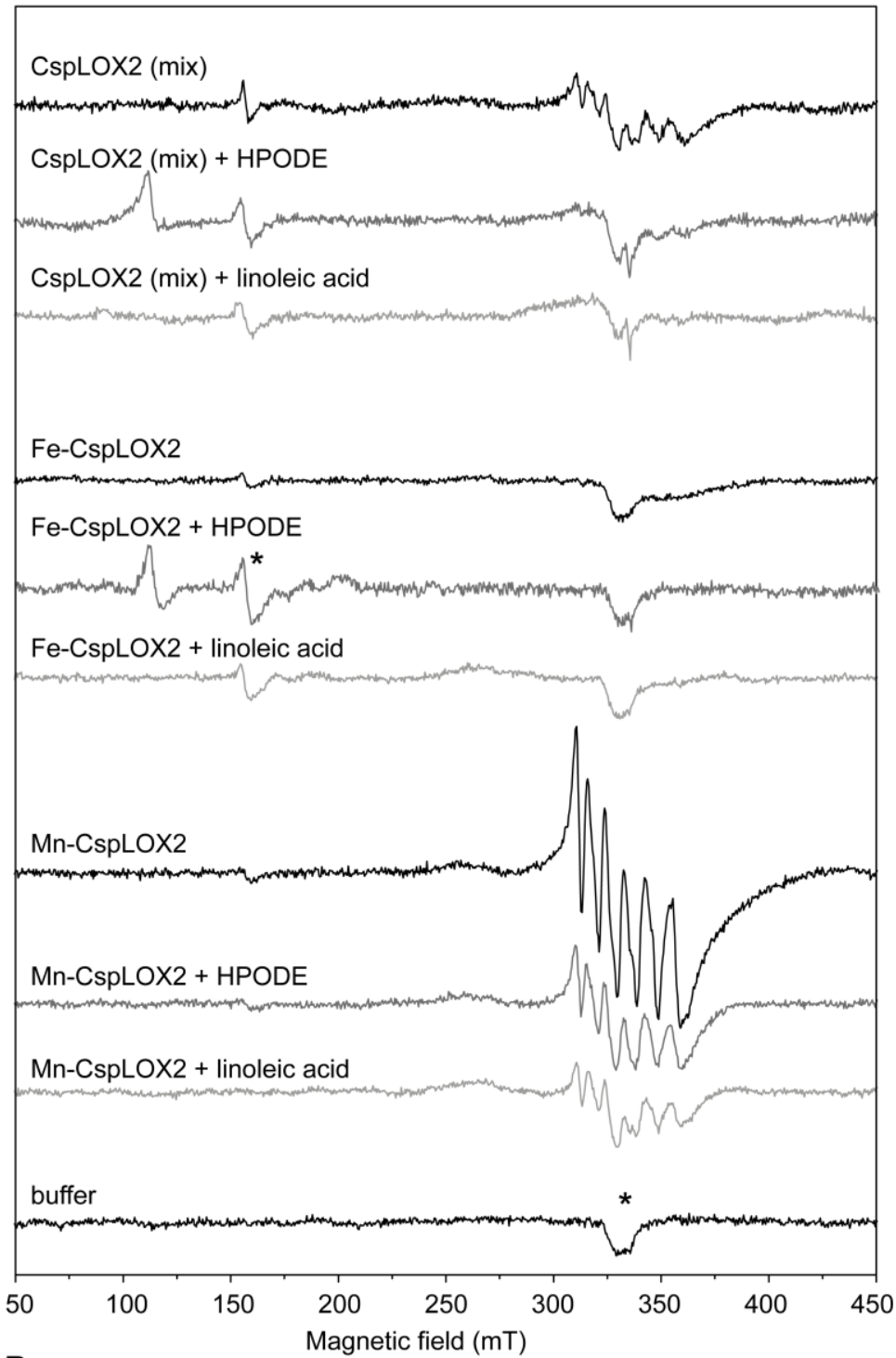

B

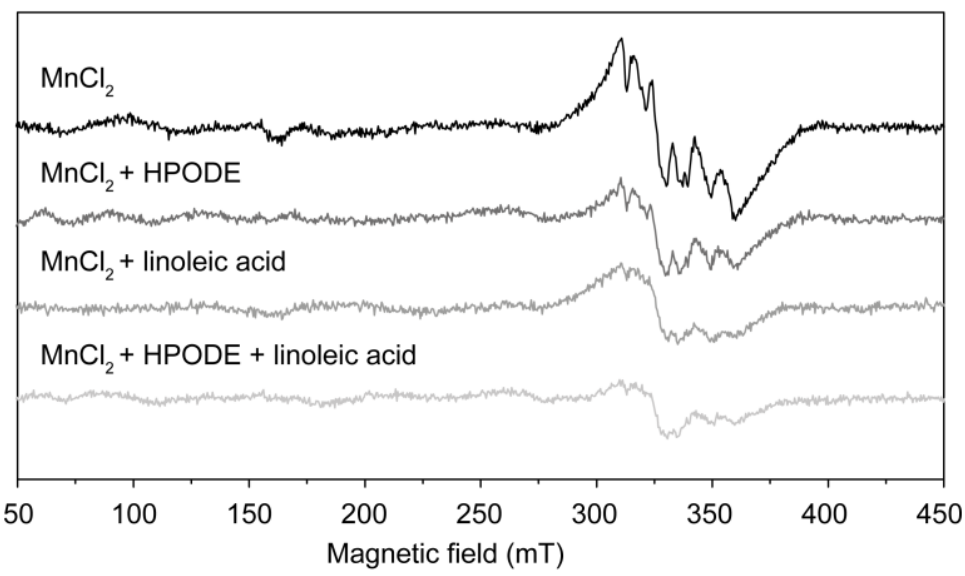

Figure 32. Comparison of the $9.4 \mathrm{GHz}$ CW-EPR spectra of metal-substituted CspLOX2 versions. Enzymes were expressed in a complex auto-induction medium (CspLOX2 (mix)) or in a defined minimal auto-induction medium supplemented either with iron (Fe-CspLOX2) or manganese (Mn-CspLOX2). (A) Spectra of different versions were recorded before and after activation with HPODE and during reaction with linoleic acid. The EPR spectrum of the buffer control is also shown. The iron impurity at $g^{\prime}=4.3$ and the signal caused by the glass impurity are shown with an asterisk. (B) In a control experiment, similar spectra as with Mn-CspLOX2 were obtained with $\mathrm{MnCl}_{2}$ only. 
Both versions were further tested for their catalytic function using the oxygen electrode. Interestingly, the activity of the iron-containing version of CspLOX2 (Fe-CspLOX2) was increased by about $20 \%$ compared to the enzyme expressed in complex auto-induction medium (CspLOX2 $(\mathrm{mix})$ ), whereas the manganese-containing version (Mn-CspLOX2) was almost inactive (Table 8). These results strongly suggest that CspLOX2 is only active with iron but not with manganese in the active site. The low amount of iron found in Mn-CspLOX2 might explain the residual activity of this variant.

Fe-CspLOX2 and Mn-CspLOX2 were also analyzed using EPR spectroscopy by Dr. Kasanmascheff. Now, upon activation with HPODE only the typical iron signal at $g_{\mathrm{xyz}} \approx 6.0,5.8$ and 2.0 was detected for Fe-CspLOX2. However, when the substrate linoleic acid was added instead of HPODEs, this signal could not be observed after one minute of incubation, which is also the case for the iron signal observed with CspLOX2(mix). As the catalytic reaction is probably completed in this time scale, it is not unexpected that the enzyme harbors Fe(II) in the active site which is EPR silent at $9.4 \mathrm{GHz}$. A small amount of iron signal observed at $g^{\prime}=4.3$ was present in all samples and was therefore attributed to contaminating iron. In contrast, Mn-CspLOX2 showed only the typical $\mathrm{Mn}$ (II) six lines EPR signal in a region close to $g=2$. When HPODE or linoleic acid was added, this signal was remarkably reduced, suggesting that $\mathrm{Mn}(\mathrm{II})$ is oxidized to $\mathrm{Mn}$ (III) which is EPR silent. Although these results would support a catalytic function of manganese, the same spectra were recorded with $\mathrm{MnCl}_{2}$ only (Figure 32B). The EPR spectra obtained with FeCspLOX2 and Mn-CspLOX2 support the ICP-AES data: Fe-CspLOX2 contains almost exclusively iron and Mn-CspLOX2 contains almost exclusively manganese.

\subsubsection{The coordination of manganese in the active site is identical to that of iron}

To confirm that manganese is indeed bound in the active site and that the protein integrity was not affected, Mn-CspLOX2 from the same purification batch used for the ICP-AES and EPR measurements was crystallized. Crystals of similar size as for wt CspLOX2 were obtained. Crystals diffracted to $2.0 \AA$ and were isomorphous with crystals of CspLOX2 expressed in complex medium. X-ray structures determined with the help of Dr. Neumann revealed that a metal atom could be detected in the characterized binding site (Figure 28A). The occupancy of iron or manganese in the active site was virtually full with $94 \%$. In comparison, the wt enzyme had an occupancy of $95 \%$ iron or manganese. Due to the minimal difference between iron and manganese in the atomic structure (manganese has 25 electrons and iron 26 electrons), both metals cannot be distinguished with the obtained X-ray diffraction data. The presence of iron or manganese in the active site with virtually full occupancy together with the ICP-AES (Table 8) and EPR (Figure 32) data point towards an integration of manganese into the active site in $\mathrm{Mn}$ CspLOX2. 
Comparison of the coordination sphere geometries of Mn-CspLOX2 with Fe-CspLOX2 revealed that they are virtually identical (Figure 33). Iron and manganese are octahedrally coordinated by His247, His262, His 449, Asn453 and lle569. The sixth position of the ligand sphere is occupied by a water or hydroxide in both structures.

A
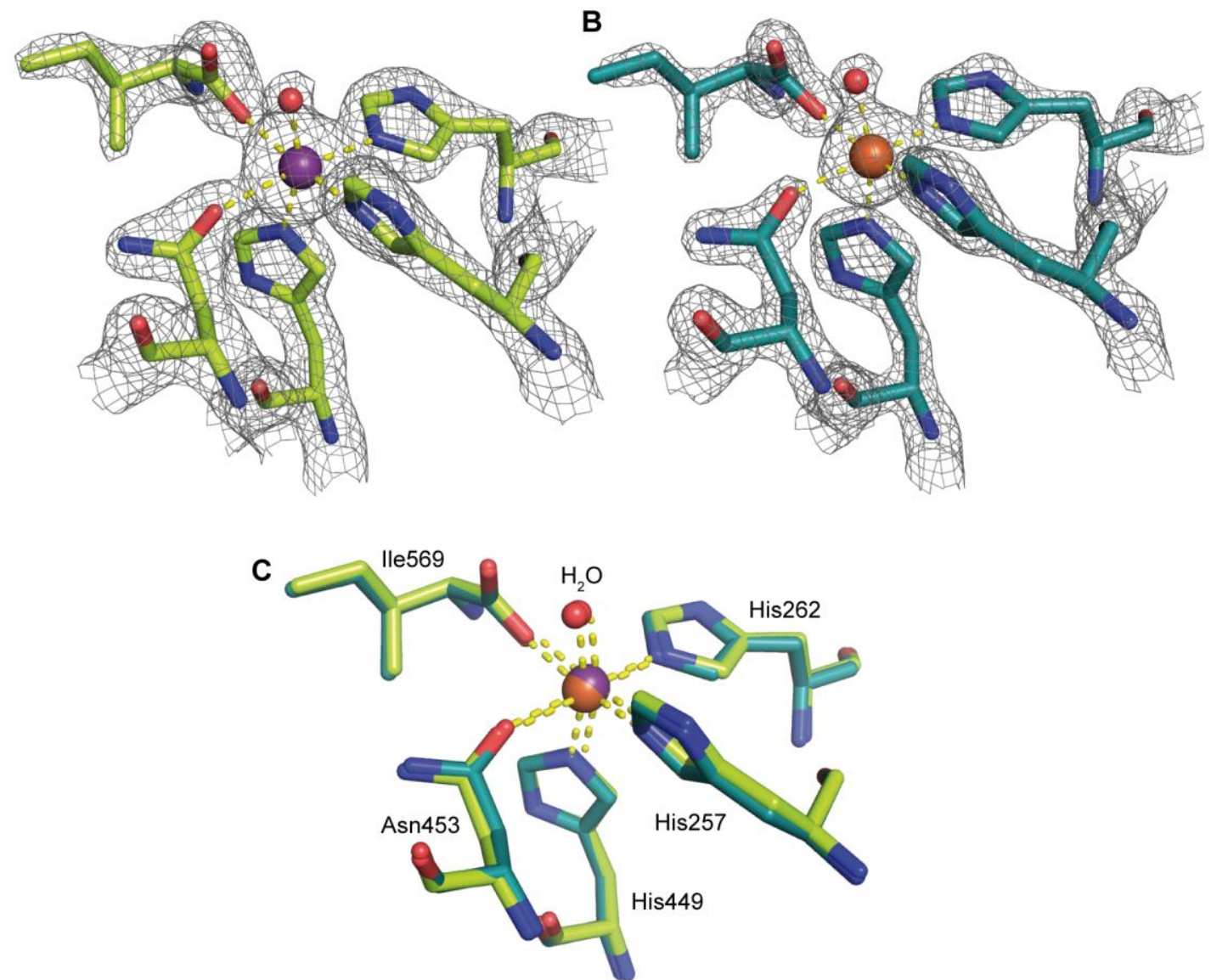

Figure 33. Coordination sphere of (A) Mn-CspLOX in comparison to (B) Fe-MnLOX. The 2mFo-DFc electron density maps are contoured at $2.8 \sigma$ for MnCspLOX2 and $4 \sigma$ for Fe-CspLOX2, respectively. (C) Superimposition of both coordination spheres reveals a virtually identical geometry. Mn-CspLOX2 is shown in light green and Fe-CspLOX2 in dark green. The catalytic metal is shown as orange (iron) or purple (manganese) sphere and the coordinated water or hydroxide as small red sphere. 


\subsection{The active site clamp controls the oxygenation specificity}

Since the formation of 11-HPODE is not restricted to LOXs with manganese as metal cofactor, it was likely that structural features of CspLOX2 are responsible for the unusual specificity. The crystal structure of CspLOX2 represents the first of a LOX with bis-allylic products. Additionally, the portion of 11-HPODE that is produced by CspLOX2 (75\% of all products, Figure 30 ) is the highest found for a LOX so far. For these two reasons, CspLOX2 is an ideal candidate to study the structural basis of this unusual specificity for bis-allylic products.

The crystal structure of CspLOX2 was consequently inspected in more detail. Although this LOX represents a mini-LOX that lacks the $\mathrm{N}$-terminal $\beta$-barrel domain, the catalytic domain strongly resembles the classical LOX domain of other enzymes. And even the boot-shaped substrate binding channel is similar to that of other LOXs like the $P$. aeruginosa 15-LOX (PDB code 4G32), the porcine leucocyte 12-LOX (PDB code 3RDE) or the P. homomalla 8R-LOX (PDB code 4QWT) (Figure 36 ). As these enzymes are unable to form the bis-allylic product in significant amounts, the differences of the CspLOX2 structure are probably more subtle. As major intra- or interdomain movements seemed unlikely to explain this reaction, the focus of this study was on the active site where the reaction takes place.

\subsubsection{Residues in the core of the active site are important for the CspLOX2 specificity} In the course of this study, more than 20 active site variants of CspLOX2 were constructed. The amino acid substitutions were specifically generated at the entrance and bottom of the putative substrate binding channel, but also at the base of the pocket which is close to the catalytic iron (Figure 34A). Mutations at the entrance of the pocket were mainly inserted to analyze interactions of the carboxyl head group of the fatty acid with positively charged amino acids, whereas mutations at the bottom of the pocket were inserted to identify what effect the depth of the substrate binding in the active site has on the enzyme specificity. The mutations close to the catalytic iron should help to elucidate how the specificity of the enzyme can be controlled by amino acids around the pentadiene, for example by steric shielding or adjustment of a potential oxygen channel. The generated CspLOX2 variants were purified and analyzed in comparison to the wt enzyme. But due to the observed fluctuations of 11-HPODE depending on the experimental conditions, initial experiments could not be compared with experiments conducted later on with the optimized reaction and extraction protocol (Chapter 3.2.1). Therefore, 18 CspLOX2 variants were purified in parallel and analyzed in comparison to wt CspLOX2. The comparability of all analyzed variants was thus as high as possible to identify the most interesting candidates with the strongest changes in specificity. 
A

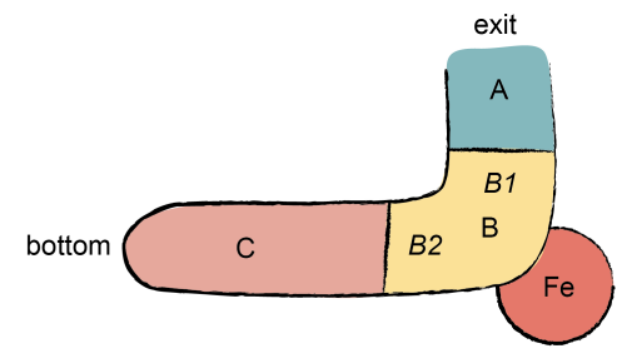

B

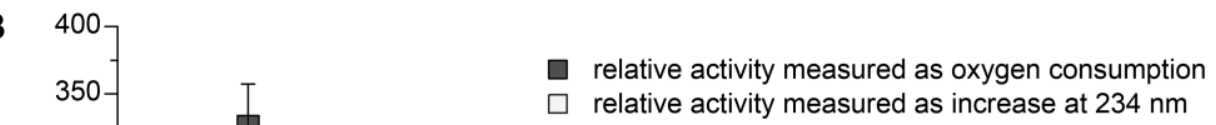

C

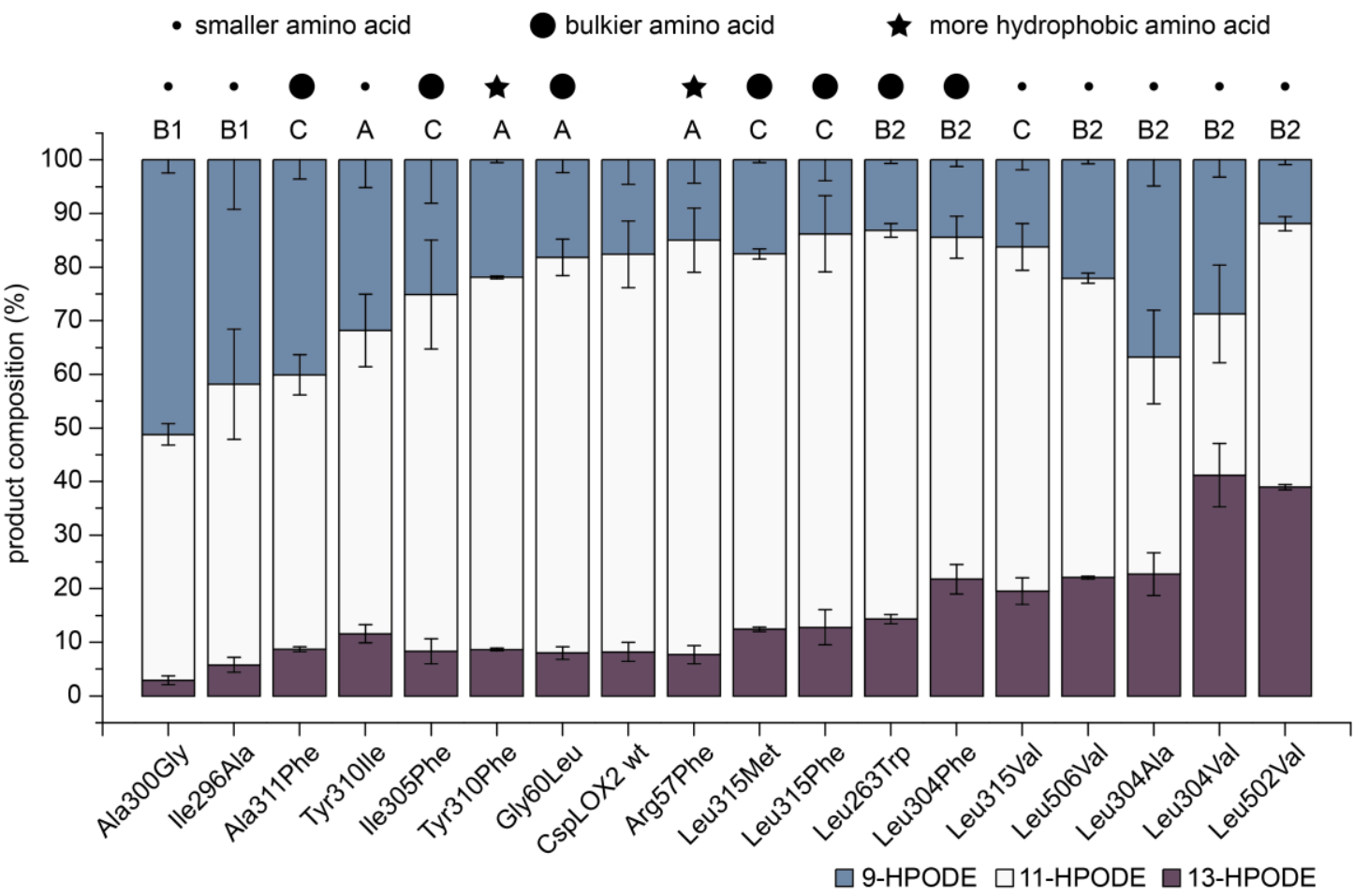

Figure 34. Comparison of 18 different CspLOX2 variants. (A) Parts of the CspLOX2 active site channel where the mutations were inserted. (B) The activities of all variants were measured as increase at $234 \mathrm{~nm}$, reflecting only the formation of conjugated double bonds or as oxygen consumption with an oxygen electrode under saturated concentrations of linoleic acid $(100 \mu \mathrm{M})$ in $200 \mu \mathrm{M}$ sodium borate buffer $\mathrm{pH}$ 9. (C) The product composition produced by the enzyme variants was evaluated by HPLC analysis. The scaling factor obtained with [1-C $\left.\mathrm{C}^{14}\right]$-linoleic acid was included in the quantification, as different products have different absorption coefficients (Supplemental Figure 2). Shown are the mean values of 3 independent experiments with standard deviations for the column below. The pictograms indicate the kind of mutation. Either the specific residue was exchanged for a smaller, bulkier or more hydrophobic residue. The letters $A, B 1, B 2$ and $C$ indicate the relative position of the amino acid exchange in the active site channel according to the depicted model $(A)$. 
All these CspLOX2 variants were purified by gravity flow IMAC and analyzed for their activity at $234 \mathrm{~nm}$ with a spectrophotometer and with an oxygen electrode (Figure 34B). As mentioned before, only conjugated products are detected at $234 \mathrm{~nm}$ (Figure 26) and the oxygen consumption is therefore a more reliable indicator for the overall activity, including the formation of the bis-allylic product. In addition to the activity, the positional specificity was determined by HPLC analysis according to the established protocol. The reaction time was adjusted to the reaction velocity of the individual variant and stopped when the first reaction phase came to an end as judged at $234 \mathrm{~nm}$.

The results are summarized in Figure 34 . The product specificities were organized from mainly 9-HPODE formation (left) to mainly 13-HPODE formation (right) (Figure 34C) and the respective activities of these variants were allocated (Figure 34B). Furthermore, the position of the mutation in the active site is shown (Figure 34A). The mutation close to the exit of the channel were categorized with the letter " $\mathrm{A}$ ", mutations close to the catalytic iron with " $\mathrm{B}$ ", whereby B1 denotes mutations "above" the iron and B2 "below" the iron, and " $\mathrm{C}$ " indicates mutations inserted at the bottom of the substrate binding pocket. Additionally, the type of mutation is indicated. These types of single amino acid substitutions include an exchange for a bulkier amino acid, for a smaller amino acid or for a more hydrophobic amino acid (Figure 34C, dots and stars).

As CspLOX2 wt produces almost equal amounts of 9- and 13-HPODE, it is located in the middle of the range from 9- to 13-HPODE specificity (Figure 34C). In this experiment, the slightest changes in the product composition occurred, when mutations were inserted near the entrance or the bottom of the substrate channel. More dramatic changes were induced when amino acids were exchanged for smaller amino acids close to the active site (denoted with the letter " $\mathrm{B}$ " and small dots), suggesting that the direct environment of the active site plays an important role for the positional specificity of the oxygen insertion on linoleic acid. But even more interesting, mutations "above" the iron (B1) correlate with a shift towards 9-lipoxygenation and mutations "below" the iron (B2) with a shift towards 13-lipoxygenation. Those variants with the most remarkable changes towards 9- or 13-HPODE also exhibited a decreased level of 11-HPODE compared to the wt enzyme. The lowest relative amount of 11-HPODE was detected for the Leu304Val variant. Exchange for alanine could not further decrease the amount of 11-HPODE and exchange for phenylalanine did not result in an increased amount of 11-HPODE (Figure 34C). The size of this residue alone can thus not explain the 11-HPODE formation. Crystal structures of the Leu304Val and Leu304Phe variant which were determined with the help of Dr. Neumann confirmed that the protein structures were only affected at the site of mutation while leaving the rest of the enzyme unchanged (Figure 35). The respective leucine residue is located opposite the catalytic iron in the kink of the substrate binding channel and is highly conserved in LOXs, suggesting an essential role for catalysis. As all LOXs require a leucine at this position, it is not intuitive, how this residue might control the formation of 11-HPODE. Nevertheless, it may control the size and form of the active site depending on the distance to the iron which is 
influenced by the distance of the arched helix (Figure 37A, in raspberry). Such slight distance changes might also be evoked by the Leu304Val substitution.

A
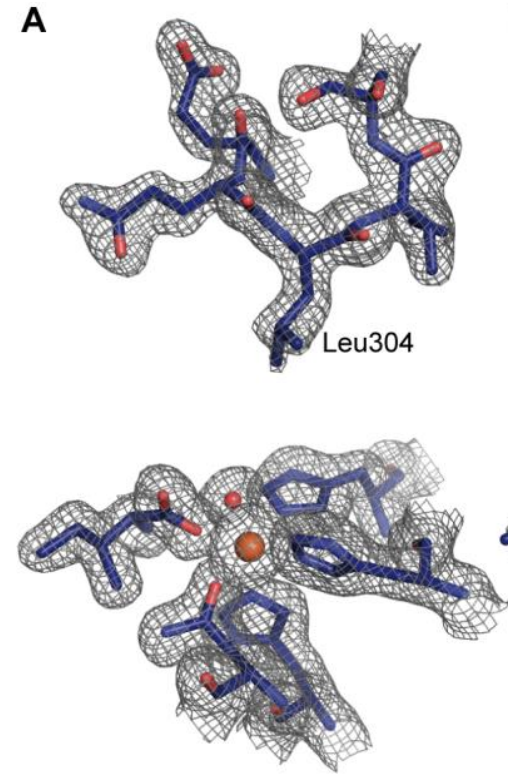

B
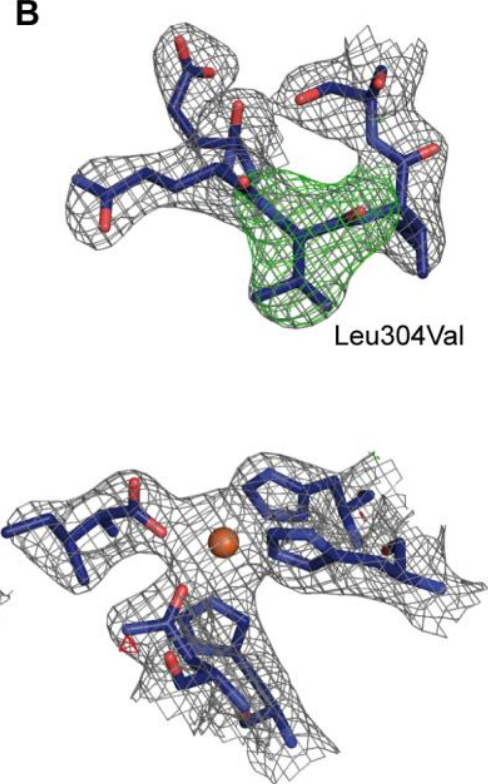

C
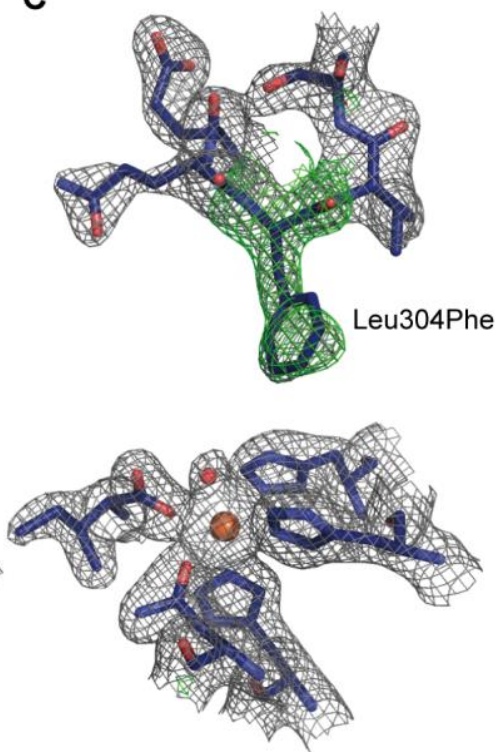

Figure 35. Structures of the Leu304Val (B) and Leu304Phe (C) variants in comparison to the CspLOX2 wt structure (A). Only the exchanged amino acid is affected by the mutation. The rest of the protein remains unchanged. The $2 \mathrm{mFo}$ $D F c$ electron density maps are contoured at $+1.5 \sigma$ and the omit maps, shown in green ( $m F o-D F c$, excluding the residue at position 304 ), are contoured at $+4 \sigma$. The catalytic iron is shown as orange sphere and the coordinated water as small red sphere.

Therefore, the distance of the $\mathrm{C}_{\mathrm{H}}$-atom of this conserved leucine to the active site iron was determined in different LOX structures available in the PDB. For most LOXs, the distance ranged from 9 - $9.9 \AA$. In CspLOX2, however, this distance was only $8.3 \AA$ (Figure 36). An even shorter distance was found in CspLOX1, although here, the active site pocket is expanded to the side, thus providing more space in another direction.

Those amino acids identified as most influential were analyzed in the crystal structure in more detail (Figure 37). These residues, including Ile296, Ala300, Leu304, Leu502 and Leu506, form together with Leu258 a tight pocket around the active site where the pentadiene has to be positioned for catalysis (Figure 37B). Due to their location around the core of the active site, these residues were named "active site clamp". Three of these residues (Ile296, Ala300 and Leu304) are contributed by the so-called arched helix (Figure 37A, in raspberry), while the other three residues (Leu258, Leu502 and Leu506) are part of other surrounding helices. 


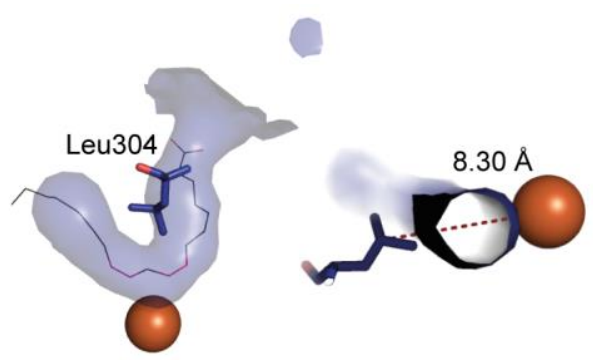

Cyanothece sp. LOX2

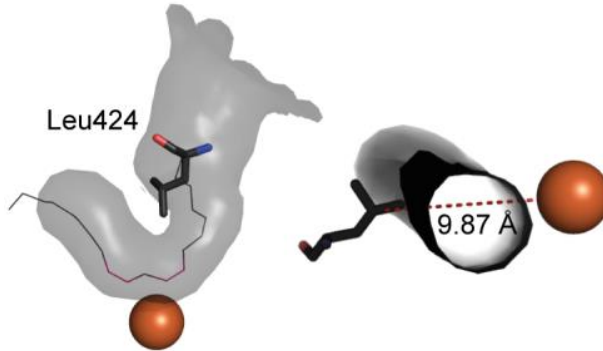

P. aeruginosa 15-LOX (4G32)
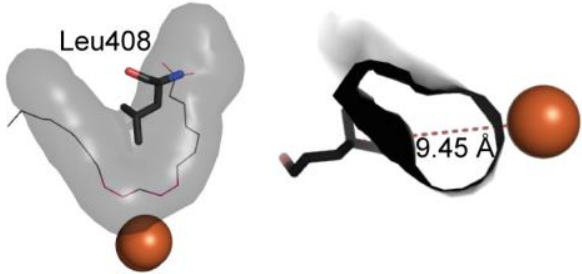

porcine leukocyte 12-LOX (3RDE)

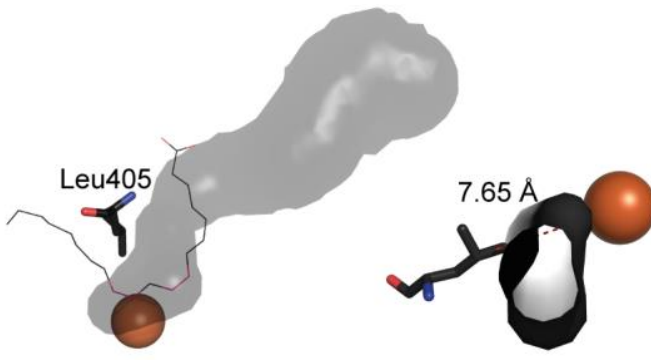

Cyanothece sp. LOX1 (5EK8)
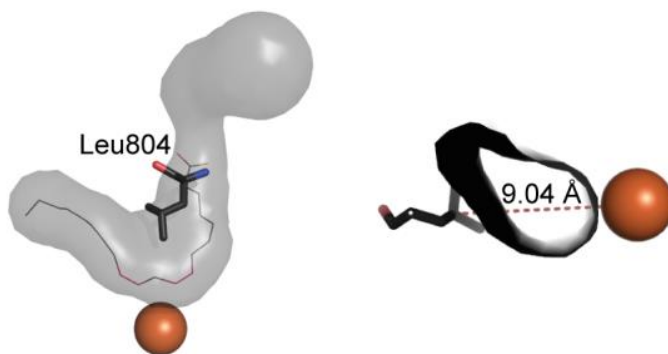

P. homomalla $8 R$-LOX (4QWT)

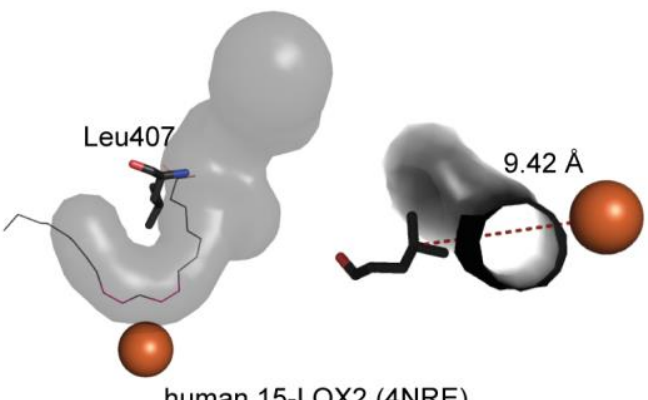

human 15-LOX2 (4NRE)

Figure 36. Diameter of putative active channels of six different LOXs. The channels were calculated with the Caver 2.0 plugin in PYMOL and are shown as gray surface (transparent) or in blue for CspLOX2. As a measure for the space at the kink region of the channel, the distance between iron (orange sphere) and $C \gamma$ atom of the conserved leucine on the opposite side of the channel (Leu304 in CspLOX2, black sticks) is shown. For a better orientation, the position of the arachidonic acid substrate soaked into the $P$. homomalla $8 R$-LOX is shown as black line with the double bonds of the reacting pentadiene system in dark red. The position was estimated from superimposition of the enzymes. PDB codes for the respective crystal structures are given in brackets.
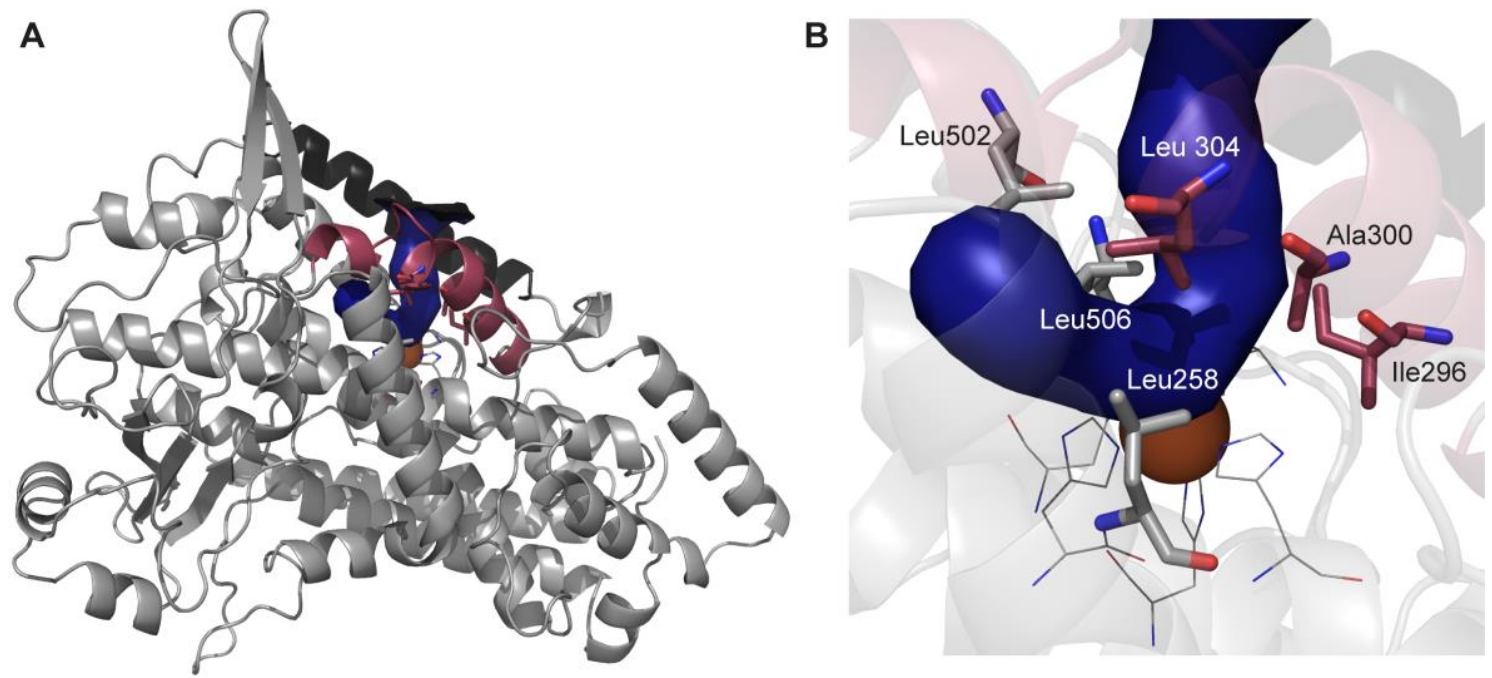

Figure 37. Location of the active site clamp in Cyanothece sp. (A) For a better orientation, the overall structure of CspLOX2 is shown with the arched helix in dark red and the helix $\alpha 2$ in black. The active site channel, visualized with the Caver 2.0 plugin for PyMOL is shown in blue and the catalytic iron as orange sphere. (B) In a zoomed-in perspective, the six residues forming the tight clamp around the active site are shown as sticks and the iron coordinating residues as lines. 


\subsubsection{The residues of the active site clamp not only influence the positional specificity, but also the stereospecificity of the products}

After having identified crucial residues around the active site that influence the positional specificity of the enzyme, it was highly interesting to get an idea of the underlying mechanism. Therefore, the stereochemistry of the hydroperoxy products produced by the active site clamp variants was determined as well. In addition to the mutations that were analyzed before, the Leu258Val variant was included.

Interestingly, not only the positional specificity could be changed by the mutations, but also the stereospecificity (Figure 38B). While Leu502Val and Leu304Val mainly produced the $S$ enantiomer of 13-HPODE, Leu506Val switched the stereospecificity of 13-HPODE to $80 \% R$. A similar observation was made for the enzyme variants that produced remarkably higher amounts of 9-HPODE. While Ala300Gly produced $9 R$-HPODE as major product with an even higher stereospecificity than the wildtype enzyme, the lle296Ala variant produced predominantly the S-entantiomer of 9-HPODE. In contrast to the conjugated 9- and 13-HPODE products, 11-HPODE was always formed in the same $R$-configuration. In general, the stereochemistry of only one product was inverted by the Ile296Ala and Leu506Val exchange. The formation of other products was almost not influenced by the mutations, suggesting that a local factor rather than a reversed orientation of the substrate is responsible for the altered product composition.

In order to understand which interactions the identified residues might have with the reacting pentadiene of the fatty acid substrate, the structure of the coral $8 R$-LOX with the bound arachidonic acid substrate (PDB code 4QWT) was used as model (Neau et al., 2014). The amino acid chain $C$ of the four chains in the asymmetric unit that contains the substrate was superimposed with CspLOX2. As shown in Figure 38A, the substrate is nicely located in the channel of CspLOX2 with the reacting pentadiene positioned within the active site clamp. This reacting pentadiene is $\mathrm{C} 8-\mathrm{C} 12$ in arachidonic acid and corresponds to $\mathrm{C} 9-\mathrm{C} 13$ on linoleic acid. The amino acids Ile296 and Ala300 seem to fix the carbon atom that would correspond to C9 on linoleic acid, while the leucines 258, 304, 502 and 506 surround the carbon atom corresponding to C13. More interestingly, Ile296 faces the side of C9 from which oxygen would need to attack to form the $S$-enantiomer while Ala300 faces the side of C9 for formation of the $R$-enantiomer (Figure 38A). These results support the hypothesis that steric shielding of the amino acid environment controls the oxygenation specificity. Around C13, the correlation between the site of mutation and stereospecificity change is less obvious. Nevertheless, Leu502 and Leu304 are rather facing the side for 13S-HPODE formation and Leu506 the side for 13R-HPODE formation (Figure 38A). Despite its proximity to $\mathrm{C} 13$, the Leu258Val exchange had only minor effects on the product composition, with only a slight decrease in 11-HPODE, but almost no effects on 9and 13-HPODE. 
A
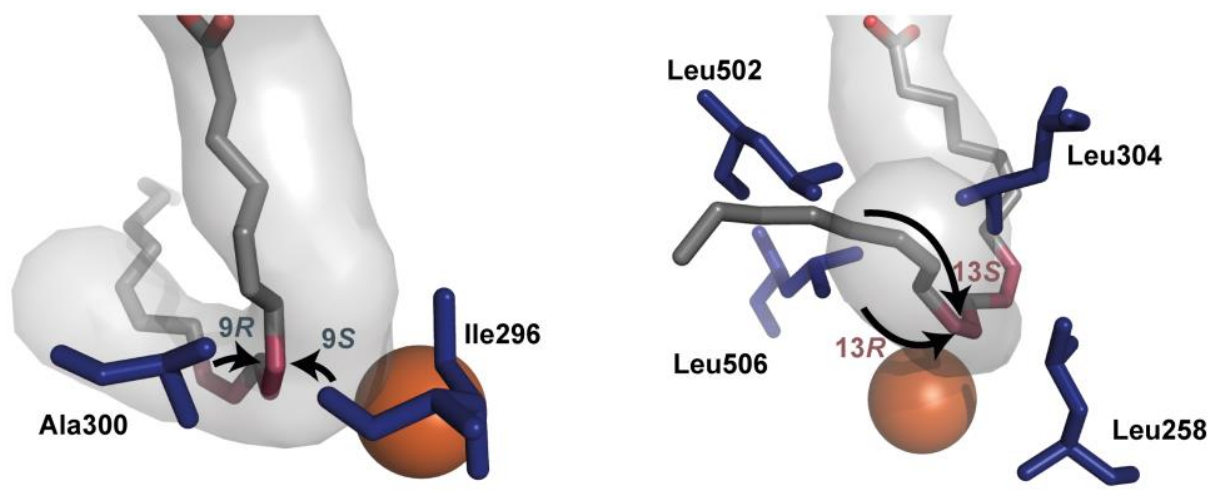

B

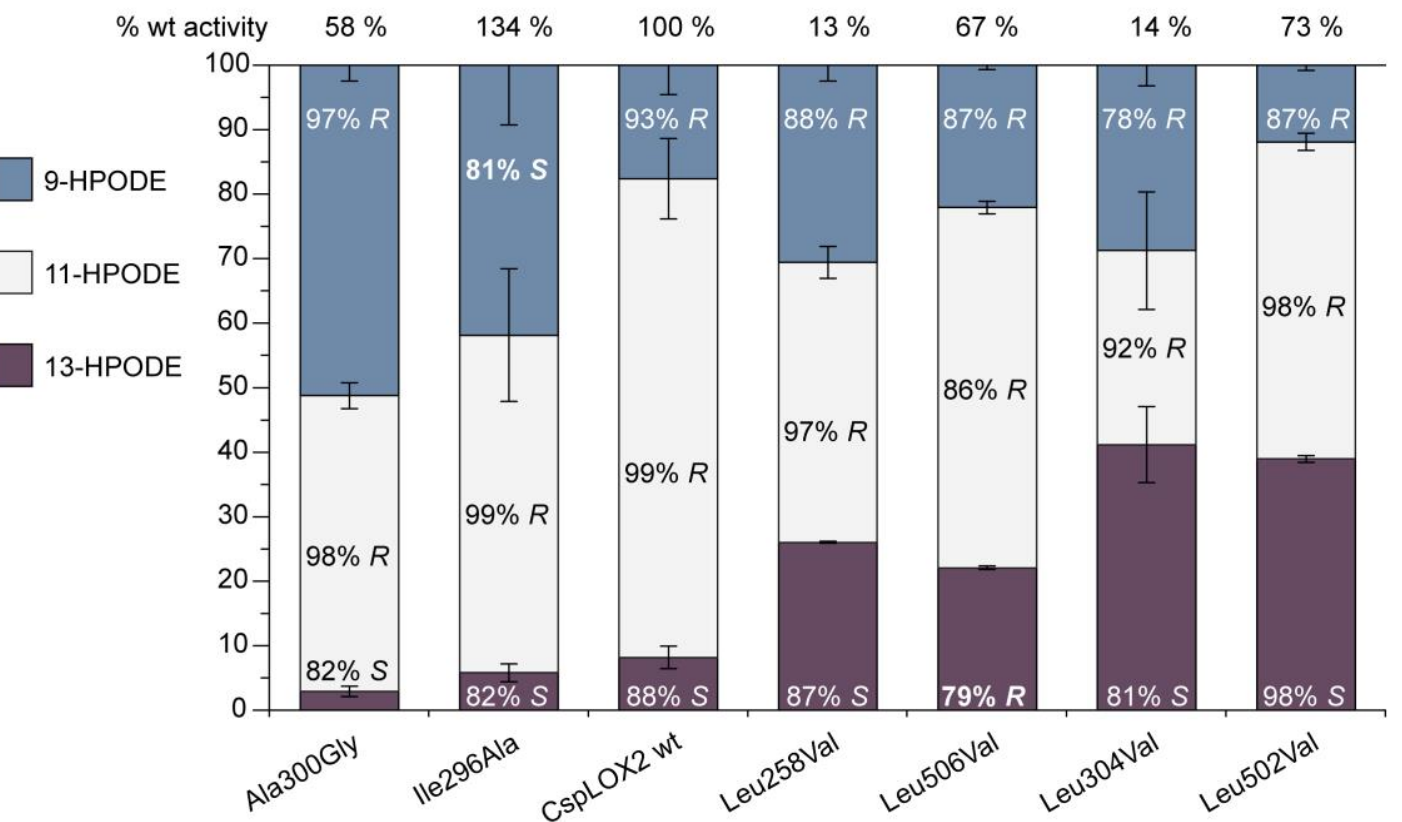

Figure 38. Mutations of the active site clamp affect the regio- and stereospecificity of CspLOX2. (A) The conformation of the arachidonic acid substrate which was crystallized in complex with the coral $8 R$-LOX (grey sticks) fits quite well into the predicted channel of CspLOX2 which is shown from the entrance part (right) and from the bottom part (left). The double bonds that belong to the reacting pentadiene are shown in dark red. The residues shown in blue belong to the active site clamp with Leu258, Leu304, Leu502 and Leu506 closer to the $n+2$ position which corresponds to C13 of linoleic acid and Ala300 and lle296 closer to the $n-2$ position (C9 of linoleic acid). (B) The residues shown in (A) were exchanged for smaller residues and the product distribution was analyzed by SP-HPLC as described for Figure 34. In addition to the distribution of the positional isomers 9-HPODE, 11-HPODE and 13-HPODE, the chirality of each product was determined by CP-HPLC and is given in percent for each product. The relative activities shown on top were determined by measuring the consumption of oxygen. The experiments were carried out in triplicates. 


\subsection{Attempts to obtain a structure of an enzyme substrate complex failed}

In order to understand the conceptual basis of the oxygenation specificity, it was desirable to obtain a structure of CspLOX2 in complex with a substrate. Although the structure of $8 R$-LOX with arachidonate provides a good model, it might be possible that the pentadiene of the substrate adopts a slightly twisted conformation in the CspLOX2 active site which could be the key to explain the 11-HPODE formation in CspLOX2, for example by radical localization.

\subsubsection{Docking}

Since crystallization of the LOX substrate complex can be very difficult due to the hydrophobic nature of the fatty acid substrate, docking was tried first using the program AutoDock Vina (Trott and Olson, 2010) in order to obtain good approximations. The search space was selected just big enough to comprise the complete substrate binding channel. This space should be large enough for substrates with 18 carbons like linoleic acid. During the docking trials, best results were obtained when 2-5 amino acids were defined as flexible residues while the rest of the protein was kept ridged. The selected flexible residues included Arg57, Asp58, Leu258, Gln303 and Leu304. Of these residues, Arg57 might be involved in the interaction with the carboxyl group of the fatty acid. Like Asp58 and GIn303, it is positioned at the entrance of the substrate channel. The obtained docking results were finally evaluated in comparison to the conformation of the crystallized substrate obtained in complex with coral 8R-LOX (Neau et al., 2014) (Figure 39). In contrast to arachidonic acid soaked into crystals of this enzyme, the reacting pentadiene was predicted in a $180^{\circ}$ flipped position in CspLOX2. Instead of the pro-S hydrogen which is oriented towards the iron in $8 R$-LOX, the pro- $R$ hydrogen is oriented towards the iron in CspLOX 2 in most docking solutions (Figure 39A). These results are in conflict with biochemical data showing that the pro-S hydrogen is abstracted from the bis-allylic methylene group in CspLOX2 (Andreou et al., 2010). It is thus unintuitive how the pro-S hydrogen should be abstracted from C11, while being oriented away from the iron cofactor (Figure 39B). In addition, the results of the mutational analysis cannot be explained with the obtained substrate models, suggesting that the docking results should not be overestimated. Instead, direct determination strategies for a crystal structure of an enzyme-substrate complex were considered. 
A
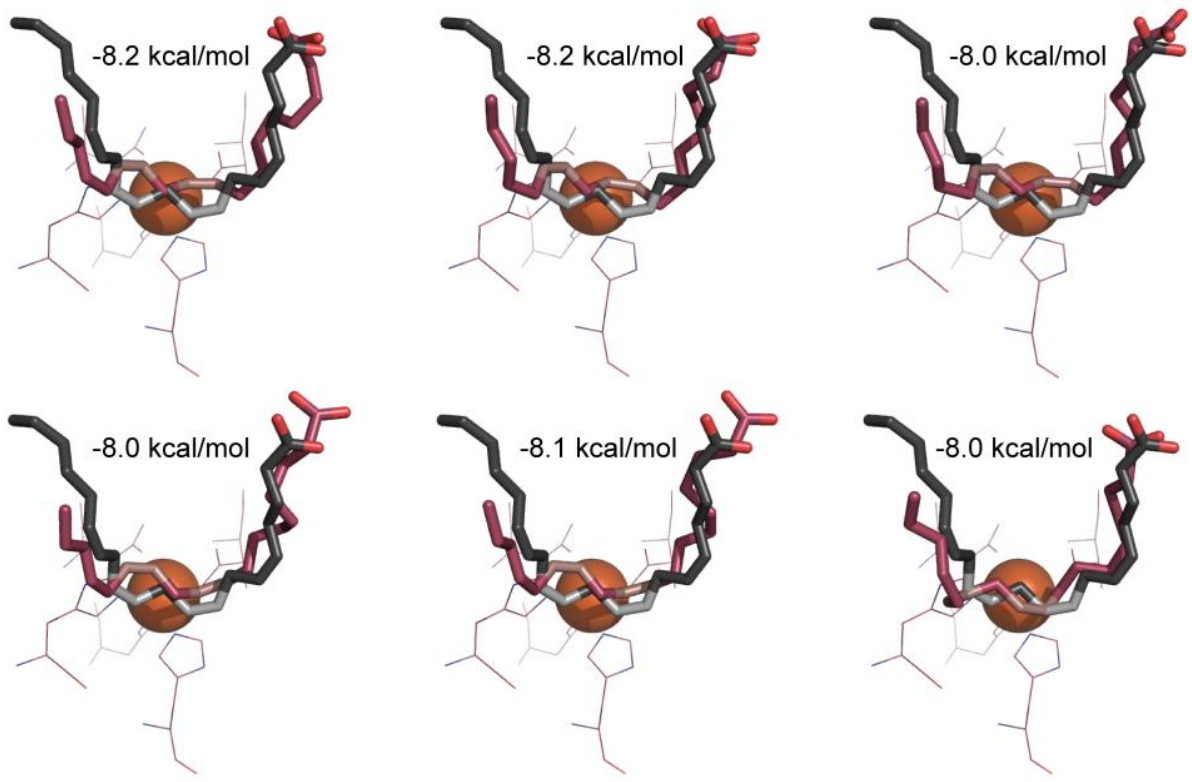

B
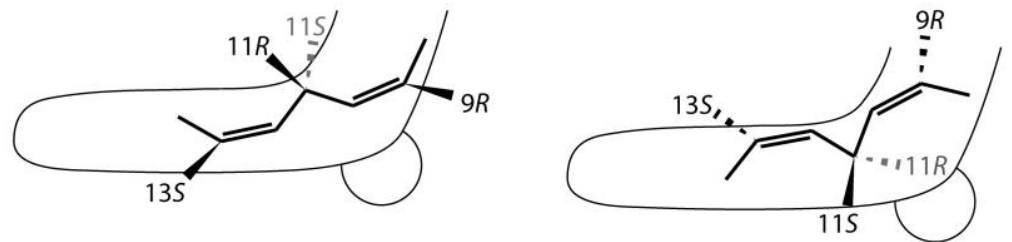

Figure 39. Best docking results of CspLOX2 with linoleic acid. (A) Shown are individual results obtained using AutoDock Vina (Trott and Olson, 2010) with 2-5 amino acids defined as flexible residues. The predicted linoleic acid conformation is shown in dark red with the double bonds of the pentadiene moiety shown in light pink. The catalytic iron is depicted as orange sphere with the coordinating residues shown as lines. For comparison, the arachidonic acid substrate crystallized in complex with the coral $8 R$-LOX is shown in black with the double bonds of the reacting pentadiene in grey. The calculated binding affinities in $\mathrm{kcal} / \mathrm{mol}$ are given for each linoleic acid conformation. (B) Depending on the conformation of the pentadiene, either the pro-S hydrogen (11S) or the pro- $R$ hydrogen (11R) is oriented towards the iron. The prochiralities of the most important positions of linoleic acid are indicated.

\subsubsection{Crystallization with substrate analogs}

Cocrystallization or soaking with real substrates often fails as substrates are converted by the enzymatic activity. The product usually exhibits a lower affinity for the active site and finally leaves the enzyme. These reasons probably also explain why no electron density of a substrate was observed in CspLOX2 in initial cocrystallization and soaking trials. To slow down the reaction, soaking was also tried at $4{ }^{\circ} \mathrm{C}$ for shorter times and with higher substrate concentrations, but still no substrate was found in the crystal structure. To circumvent this problem completely, substrate analogs that cannot be converted by LOXs were chosen as ligands.

Fatty acids that cannot be converted as they have only one double bond and therefore no pentadiene system are for instance oleic acid and vaccenic acid. Of the two, vaccenic acid was also found as ligand in the P. aeruginosa 15-LOX (Garreta et al., 2013). Both fatty acids have 18 carbon atoms and thus the same length as linoleic acid (Table 9). These substrate analogs are thus suitable compounds to study the potential interaction of the carboxyl head group with 
amino acids of CspLOX2 as well as the overall substrate positioning. Nevertheless, no electron density could be detected for these compounds.

Another problem is that fatty acids have only a low solubility in aqueous solutions due to their nonpolar character. It might thus be difficult to reach a sufficiently high concentration of the ligand in order to obtain a complex with the enzyme. Therefore, compounds with a higher solubility might be beneficial. The substrate analog tetraethylene glycol monooctyl ether (C8E4) which was successfully found in the active site of the human 15-LOX (Kobe et al., 2014) appeared to be a suitable substrate analog. In the human 15-LOX (PDB code 4NRE), it was found accidently in the active site after an additive screening (Kobe et al., 2014). The detergent occupies the active site with the more polar tetraethylenglycol part of the molecule bound deep in the active site and the octyl chain closer to the entrance of the binding channel. The detergent has also been shown to be an inhibitor for the human 15-LOX (Kobe et al., 2014). Before starting the crystallization of CspLOX2 with C8E4, the detergent was thus tested for its inhibitory effect on the CspLOX2 reaction. Although the compound had only a slight inhibitory effect on wt CspLOX2, it was a potent inhibitor for the Leu304Phe variant (Figure 40 ). The inhibitory constant $K_{\text {I }}$ of C8E4 was $580 \mu \mathrm{M}$ for this variant. At this concentration, the reaction rate with $100 \mu \mathrm{M}$ linoleic acid decreased by a half. The cocrystallization was hence not only tried with the wt enzyme, but also with the Leu304Phe variant. Nevertheless, none of the trials resulted in sufficient electron density in the active site that could correspond to the inhibitor.

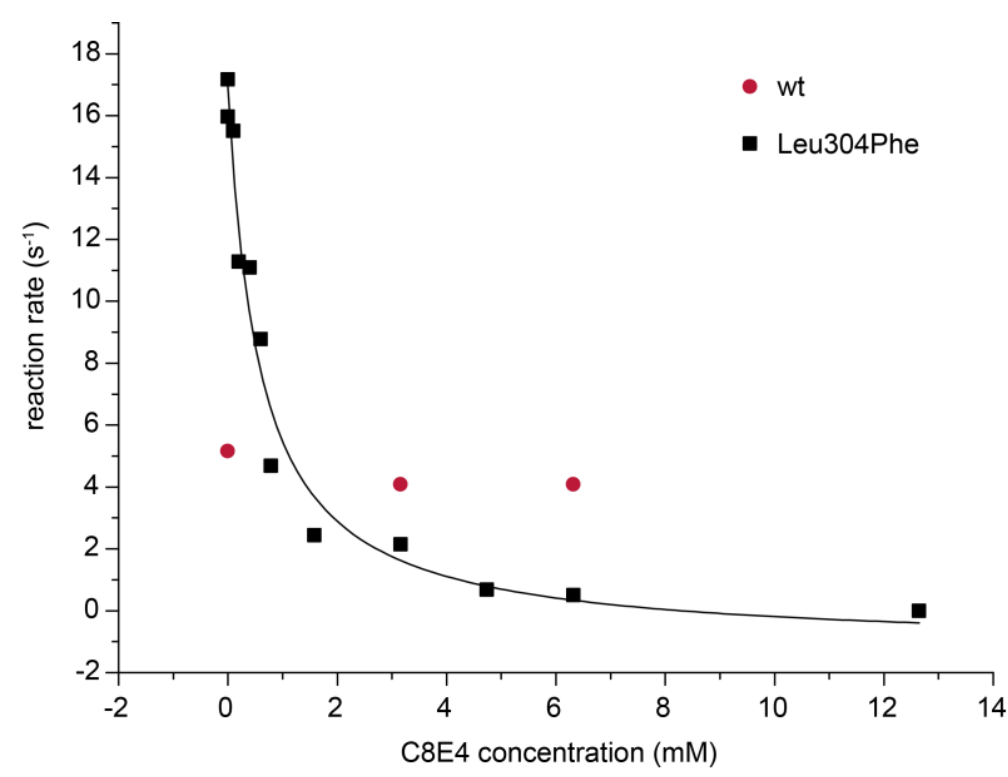

Figure 40. Inhibition of CspLOX2 wt and Leu304Phe with tetraethylene glycol gonooctyl ether (C8E4). Enzymes were purified by IMAC and incubated with $100 \mu \mathrm{M}$ linoleic acid in the presence of varying C8E4 concentrations. The enzymatic activity was determined at $234 \mathrm{~nm}$ using a spectrophotometer and plotted against the C8E4 concentration. 
The most interesting information for our studies would be the position of the reacting pentadiene in the CspLOX2 active site to elucidate which function the active site clamp may fulfill and whether the pentadiene adopts a twisted conformation. Consequently, it would be best to use substrates which contain a pentadiene system, but are no functional substrates. Fluorinated linoleic acid which contains two fluorine atoms at C11 instead of hydrogens would be a perfect candidate, but is not yet available. Thus, the special activity of CspLOX2 was applied to produce an alternative substrate analog. While 11-HPODE still serves as substrate, even though it is converted at a much slower speed (Figure 28B), its hydroxide derivative 11-HODE (Table 9) is no substrate, as incubation of 11-HODE with CspLOX2 did not lead to an increase in absorbance at $234 \mathrm{~nm}$ (data not shown). At the same time, 11-HODE has enough similarity with the substrate and even contains the two double bonds found in the pentadiene system. Nevertheless, soaking with this substrate analog was again not successful.

\subsubsection{Substitution of the catalytic metal}

An alternative strategy to obtain an enzyme substrate complex is to use an inactive variant that is structurally almost indistinguishable from the wt enzyme. As shown before, a manganesesubstituted CspLOX2 variant could be obtained that was virtually inactive (Chapter 3.3.1). This variant was a perfect candidate for soaking with linoleic acid or arachidonic acid. Although welldiffracting crystals could be obtained with Mn-CspLOX2, which confirmed the structural integrity of the substituted variant (Figure 33), no electron density that could correspond to linoleic acid or arachidonic acid was found in the active site.

\subsubsection{Anaerobic crystallization}

The crystal structure of the coral $8 R$-LOX with bound arachidonic acid was recently obtained by anaerobic crystallization and soaking with arachidonic acid (Neau et al., 2014). In a last trial, CspLOX2 was hence crystallized under anaerobic conditions in the laboratory of Prof. Dr. Marcia Newcomer (Department of Biological Sciences, Louisiana State University, Baton Rouge, USA). The principle of this experiment is that one of the substrates, in this case oxygen, is omitted from the crystallization conditions. The fatty acid substrate will thus bind in the active site of the LOX but not react. Crystals appeared after 2-5 days and were soaked with arachidonic acid and linoleic acid under anaerobic conditions for different timescales ranging from a few minutes to $20 \mathrm{~h}$. Moreover, crystals were also obtained by cocrystallization with up to $1 \mathrm{mM}$ linoleic acid or arachidonic acid. However, again none of the 16 crystals that were tested showed sufficient electron density in the active site that could belong to a substrate molecule (Table 9). Instead, the structures resembled the holo-enzyme of CspLOX2 obtained at ambient oxygen levels. 


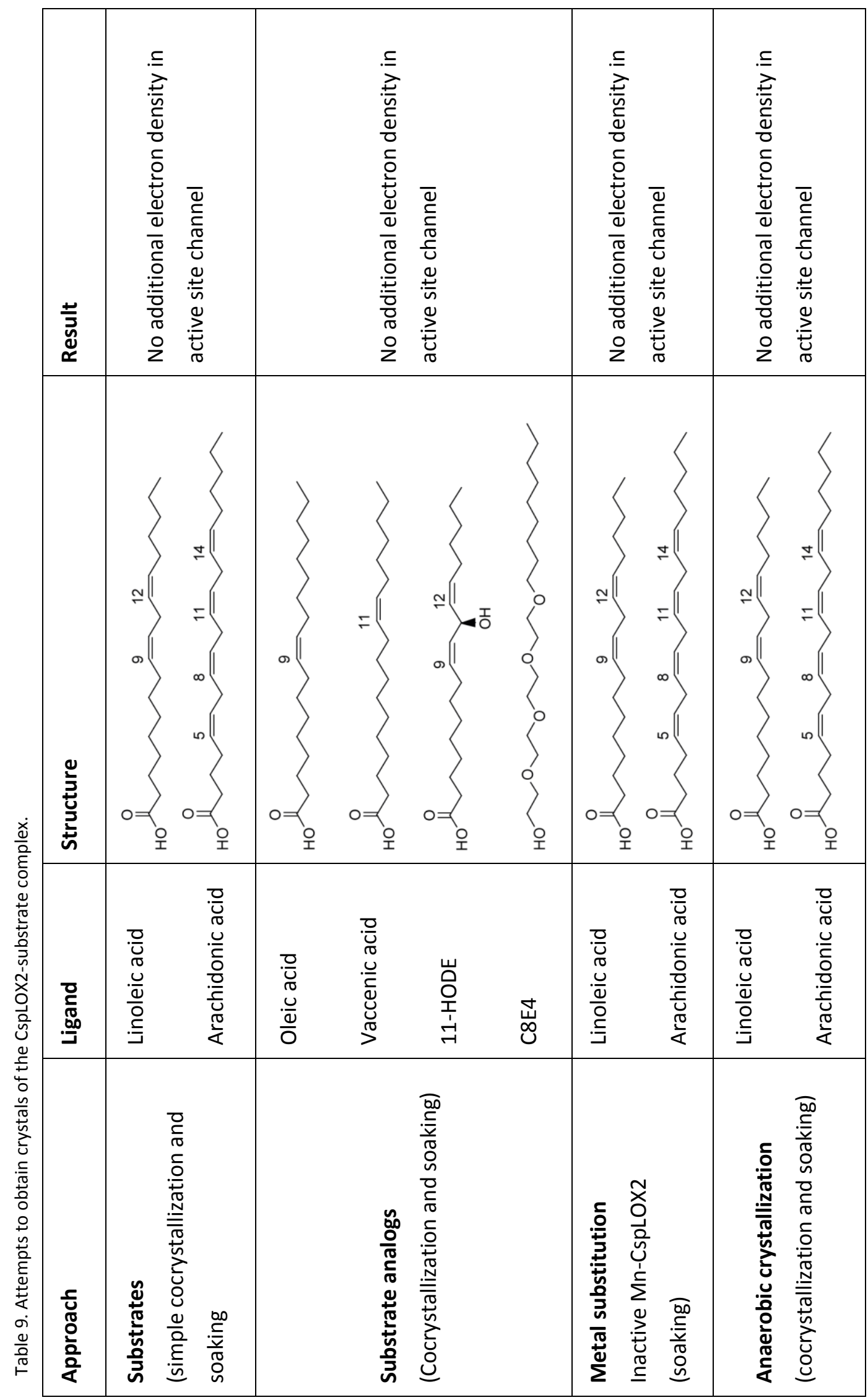




\subsection{Mimicking the CspLOX2 active site induces 11-HPODE in another LOX}

A possibility to get a better idea of the factors involved in oxygen insertion at C11 of linoleic acid is to change a classical LOX which does not produce 11-HPODE, but only conjugated hydroperoxides, into a LOX that forms bis-allylic products. For this gain of function approach CspLOX1 was chosen, as this enzyme originates from the same organism, produces only $9 R$ HPODE from linoleic acid with a very high specificity, a crystal structure is available (Newie et al., 2015) and the diameter of the channel defined by the distance of the catalytic iron to the conserved leucine is comparably small (Figure 36 ).

\subsubsection{Exchange of two amino acids in CspLOX1 is sufficient for 11-HPODE formation}

To identify which mutations might be necessary in CspLOX1 to induce 11-HPODE formation, the amino acids of the CspLOX2 active site clamp were compared with the respective residues of CspLOX1 (Table 10). Of these residues, lle296, Leu304 and Leu506 of CspLOX2 are conserved and correspond to lle397, Leu405 and Leu621 in CspLOX1. On the other hand, Leu258, Ala300 and Leu502 of CspLOX2, are occupied by Tyr360, Gly401 and Ile617 in CspLOX1, respectively. These residues were hence exchanged. In addition to these three residues, Phe413 which seems to block the bottom of the channel was replaced by the respective alanine residue found in CspLOX2 (Figure 41).

Table 10. Comparison of active site clamp residues in CspLOX1 and CspLOX2. Residues differing in both enzymes are printed in bold. In addition to the active site clamp residues, Phe413 was exchanged. The oxygenation specificity on linoleic acid that is associated with the individual residues of CspLOX2 is marked with an asterisk.

\begin{tabular}{l|cccccc|c|c}
\hline & $9 S^{*}$ & $9 R^{*}$ & \multicolumn{3}{c}{$13 S^{*}$} & $13 R^{*}$ & & Main product \\
\hline CspLOX2 & Ile296 & Ala300 & Leu304 & Leu502 & Leu506 & Leu258 & Ala311 & $11 R$ \\
CspLOX1 & Ile397 & Gly401 & Leu405 & Ile617 & Leu621 & Tyr360 & Phe413 & $9 R$
\end{tabular}

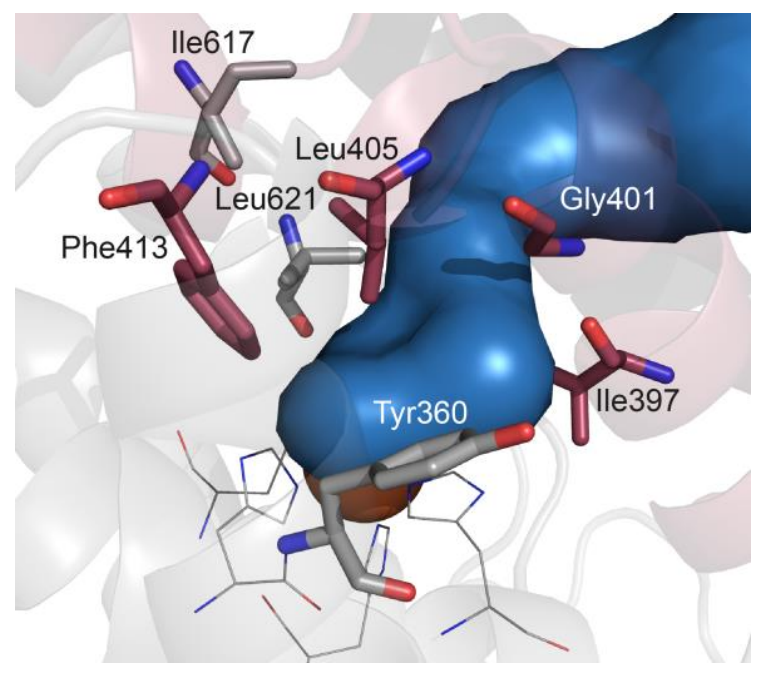

Figure 41. Active site clamp residues of CspLOX1. The residues of the active site clamp are shown as sticks, the putative substrate binding channel in light blue, the iron coordinating residues as lines and the catalytic iron as orange sphere. 
$\begin{array}{lllllllllll}\text { A marker } & 1 & 2 & 3 & 4 & 5 & 6 & 7 & 8 & 9 & 10\end{array}$

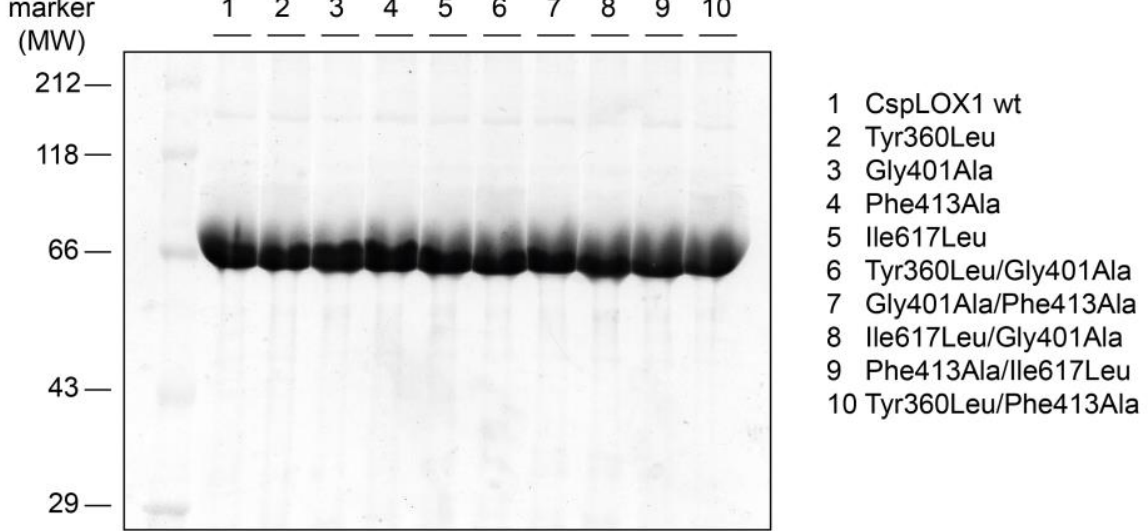

B

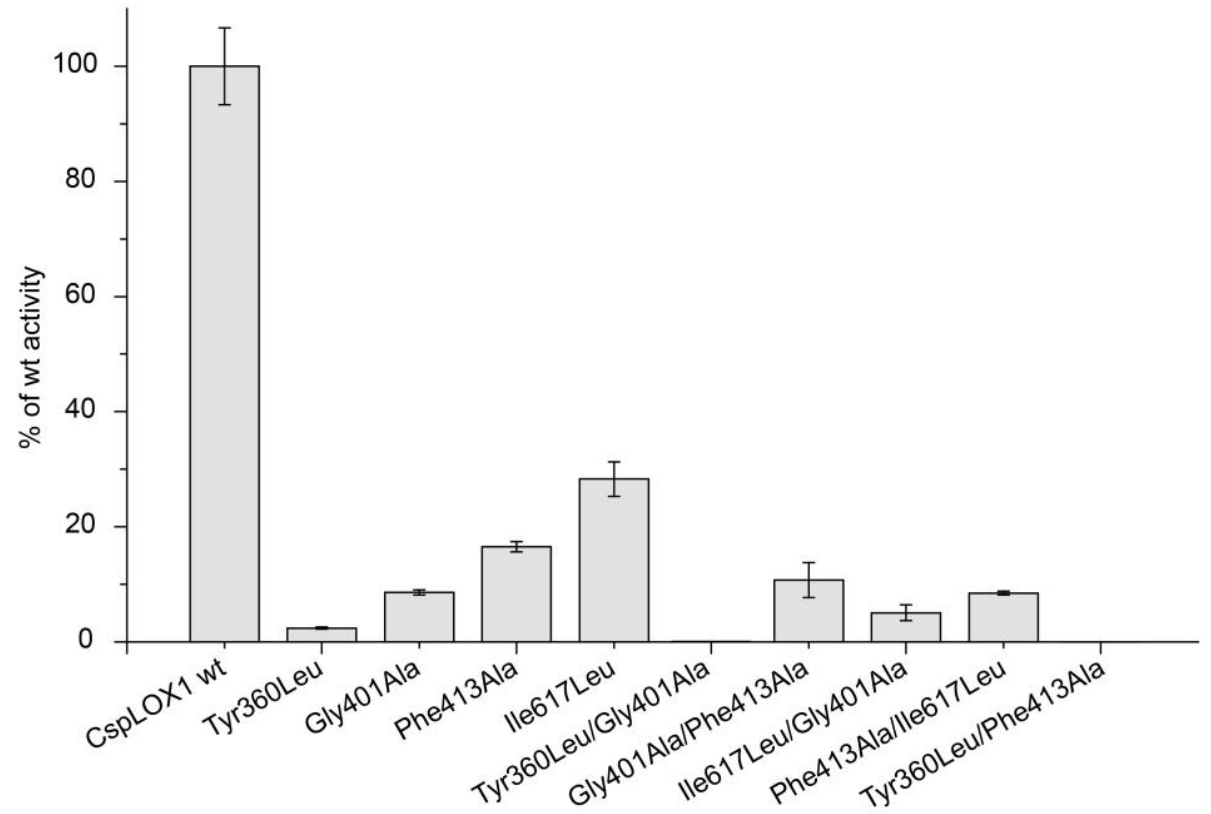

C

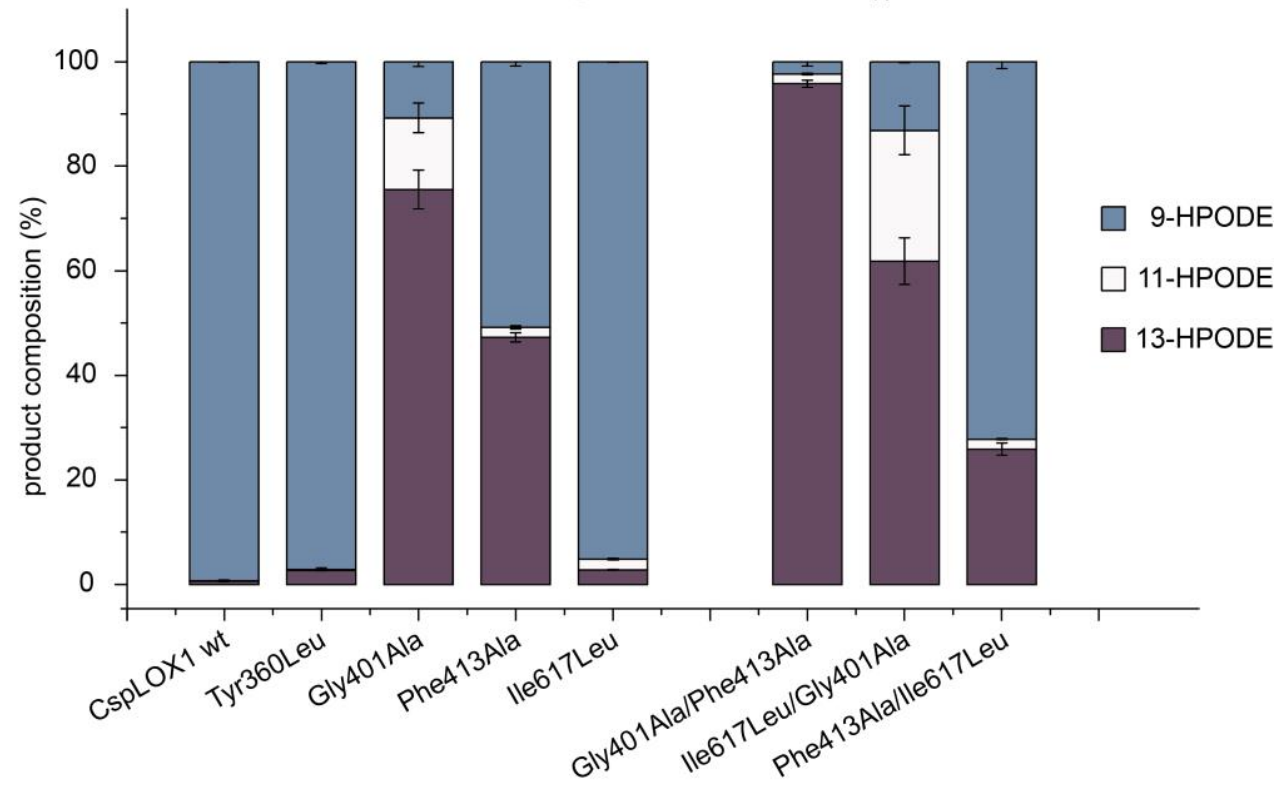

Figure 42. Activity and oxygenation specificity of CspLOX1 variants containing amino acid substitutions in active site. (A) The variants were purified by IMAC and their purity was confirmed by SDS-PAGE. (B) The relative activity of the variants was determined at $234 \mathrm{~nm}$ with a spectrophotometer. (C) The positional specificity of oxygen insertion is shown as relative amount of 9-, 11- and 13-HPODE as determined by SP-HPLC. The scaling factors (Supplemental Figure 2) were used for the quantification at $202 \mathrm{~nm}$ (11-HPODE) and $234 \mathrm{~nm}$ (9- and 13-HPODE). 
The protein variants were purified by IMAC and analyzed by SDS-PAGE for their purity (Figure 42A). Subsequently, the protein variants were tested for their catalytic activities with linoleic acid. The variants with single amino acid substitutions retained only about $2-28 \%$ of the wt activity, with Tyr360Leu causing the most severe decrease in activity. Combination of Tyr360Leu with other exchanges resulted in a complete loss of catalytic activity (Figure 42B). CspLOX1 variants exhibiting at least $2 \%$ of wt activity were tested for their product specificity with linoleic acid. While only minor changes in the product composition could be observed when Tyr360 or lle617 were exchanged, the Phe413Ala variant lost its specificity and produced almost equal amounts of 9-HPODE and 13-HPODE (Figure 42C).

The most interesting effect exhibited the Gly401Ala variant. The positional specificity of this variant was changed from 9-lipoxygenation to mainly 13-lipoxygenation. But even more surprising, the variant indeed produced a compound that coelutes with 11-HPODE (Figure 43). Furthermore, the amount of this compound could even be increased when the Gly401Ala exchange was combined with lle617Leu. The identity of the peak was confirmed to be 11-HPODE by comparison of the UV-spectrum and LC-MS analyses of the hydroxyl derivative of the compound by Dr. Kirstin Feußner (Figure 44). These results show that it is indeed possible to induce 11-HPODE formation in a 9R-LOX like CspLOX1 by only one or two amino acid exchanges.

Interestingly, Gly401 is assumed to be close to C9 on linoleic acid, whereas lle617 is probably closer to C13 (Table 10). Consequently, both residues may limit the oxygen access to C9 and C13, thereby leaving only the bis-allylic position for oxygen insertion. Together with the results from CspLOX2, a tight and narrow substrate binding channel seems to support 11-HPODE formation, while a wider channel results in the formation of only conjugated hydroperoxides.

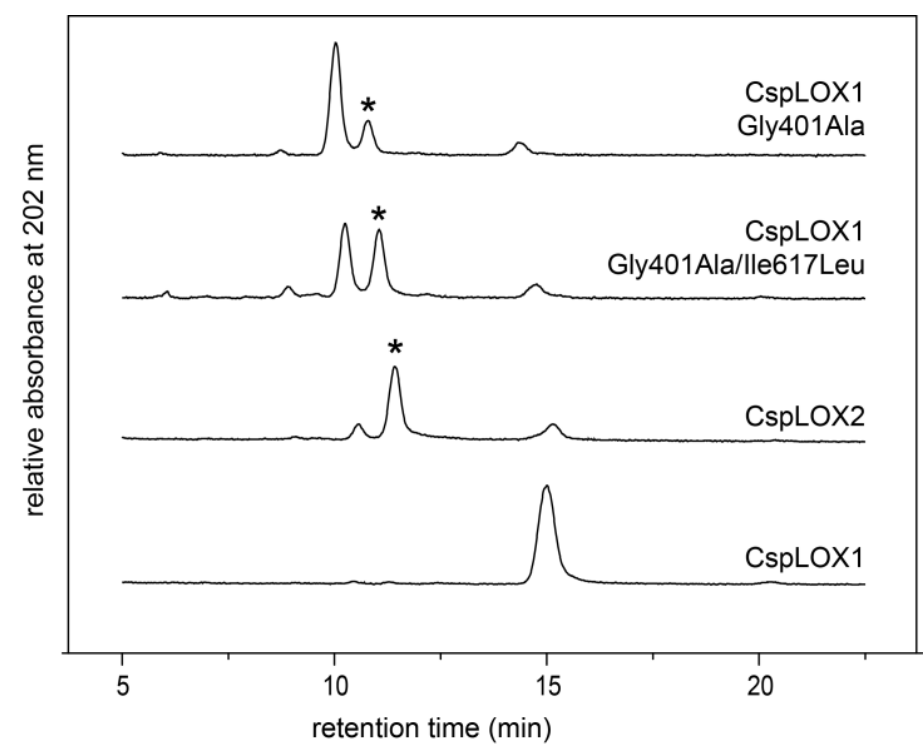

Figure 43. Two amino acid substitutions in CspLOX1 induce 11-HPODE production. Chromatograms of the SP-HPLC analyses of products formed by CspLOX2, CspLOX1 and the Gly401lle as well as the 617Leu/Gly401Ala variant of CspLOX1 are shown. While CspLOX1 only produces 9-HPODE, the lle617Leu/Gly401Ala variant produces significant amounts of the bis-allylic 11-HPODE that is characteristic for CspLOX2. The peak corresponding to 11-HPODE is highlighted with an asterisk. The other peaks eluting at around $10 \mathrm{~min}$ and $15 \mathrm{~min}$ correspond to 13 HPODE and 9HPODE, respectively. 

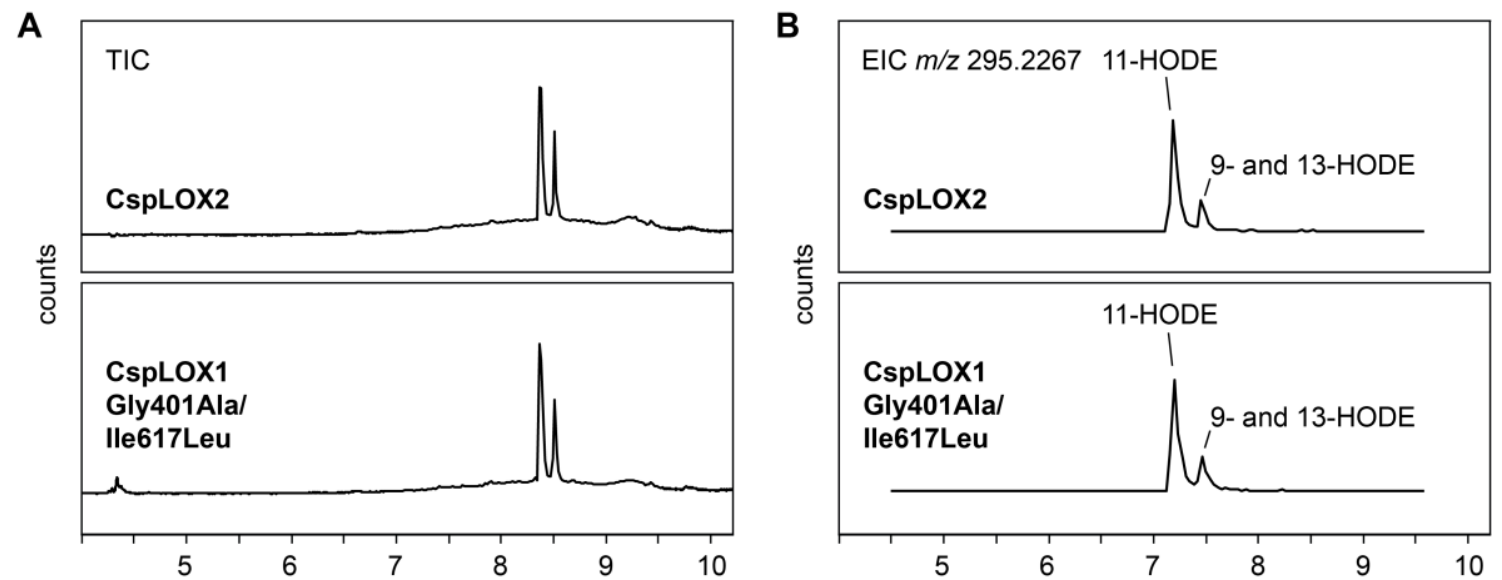

C 11-HODE (retention time $7.14 \mathrm{~min}$ )

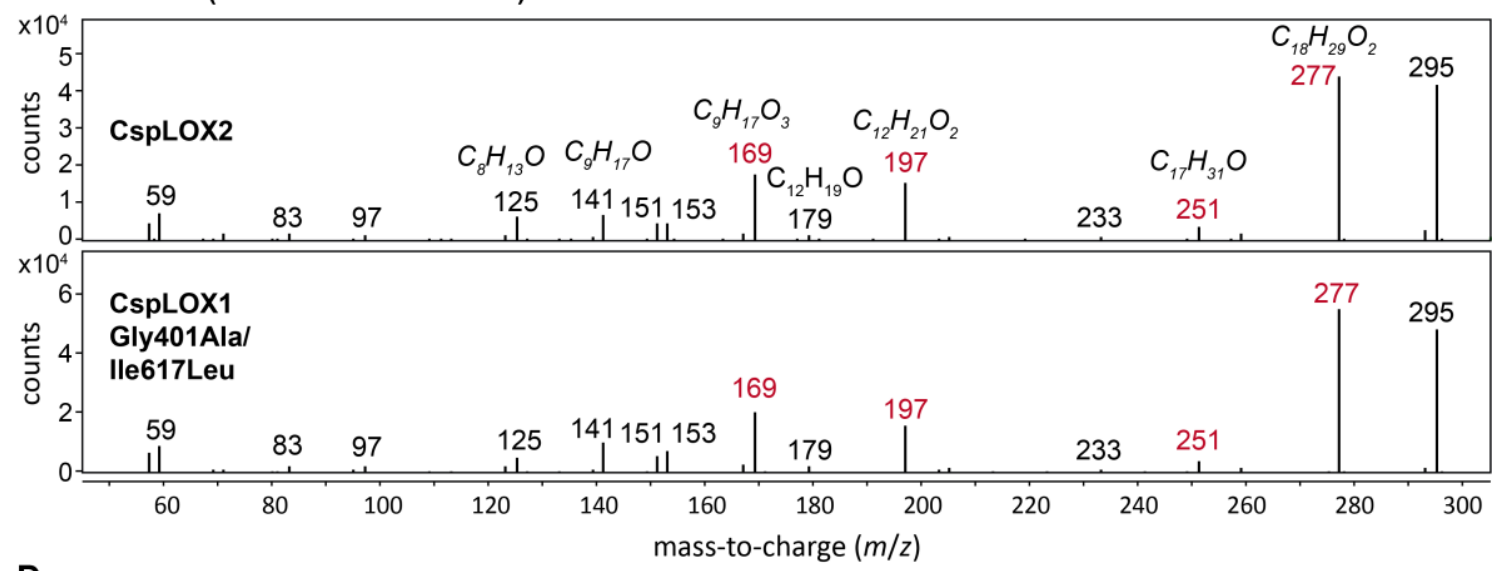

9- and 13-HODE (retention time $7.35 \mathrm{~min}$ )
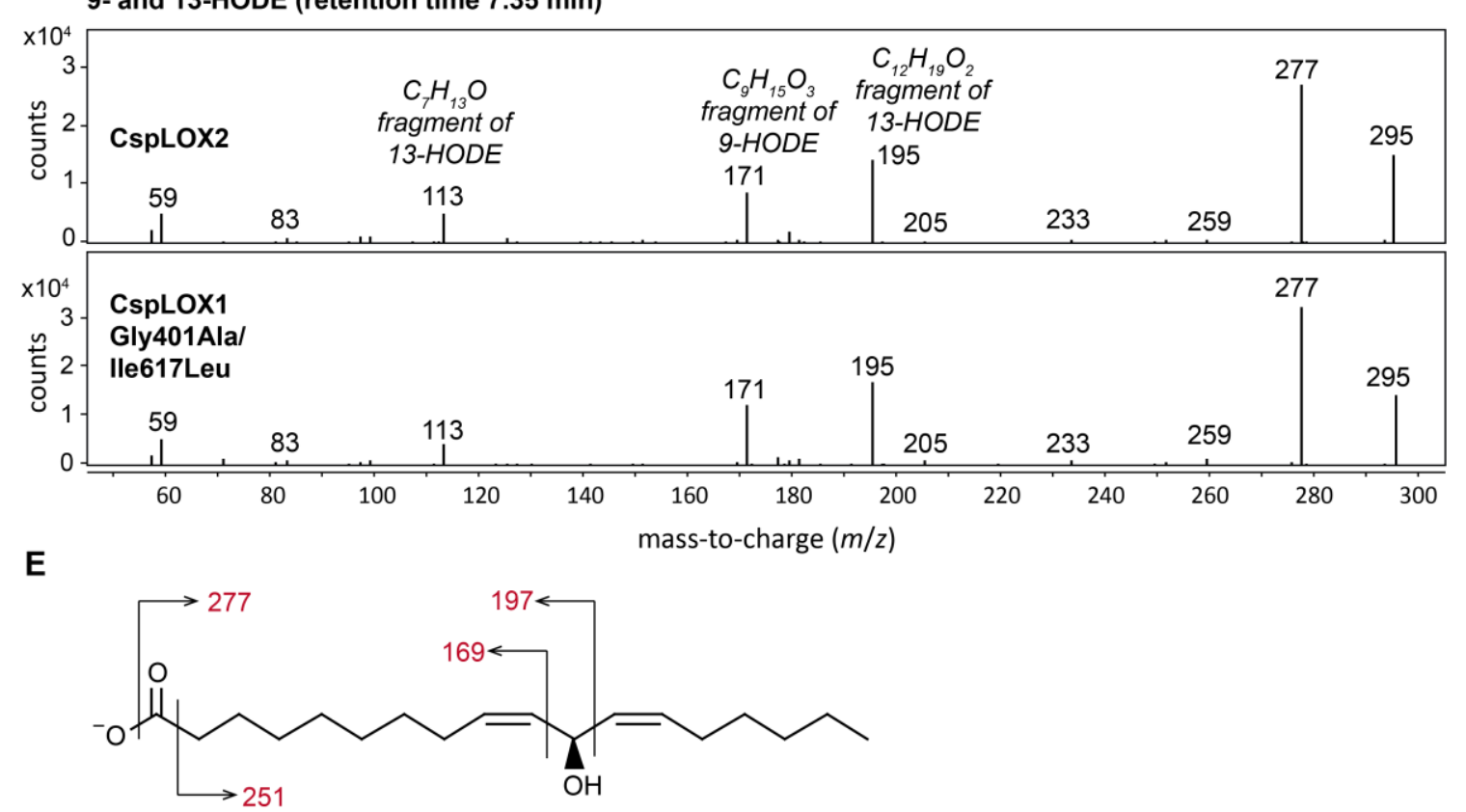

Figure 44. Confirmation of the 11-HPODE species produced by the Gly401Ala/lle617Leu variant of CspLOX1. 11HPODE produced by CspLOX2 and the CspLOX1 variant were reduced to hydroxides, purified by HPLC and subjected to UHPLC-QTOF MS. (A) The total ion chromatogram (TIC) is shown for both samples analyzed in the negative ionization mode and (B) the corresponding extracted ion chromatogram (EIC) at $\mathrm{m} / \mathrm{z} 295.2267$. (C) The high resolution mass spectrum (resolution $<2 \mathrm{ppm}$ ) for the first peak eluting at 7.14 min corresponds to $11-\mathrm{HODE}$, while (D) the mass spectrum for the peak eluting at 7.35 min corresponds to 9- and 13-HODE. The exact mass information of the MS/MS fragments was used for deducing the elemental composition. (E) Fragmentation pattern of 11-HODE and the resulting $m / z$ signals 
Since Gly401 occupies the residue that is also known as Gly/Ala switch (Coffa and Brash, 2004), the stereospecificity of 13-HPODE formed by the Gly401Ala variant was also determined for this variant. In line with the accepted model, the exchange of this residue switched the specificity from $9 R$ with glycine at this position to mainly 13S-HPODE with alanine at this position (Figure 45). Since the main focus of this approach was to induce 11-HPODE formation in CspLOX1, the stereospecificites of the products produced by other CspLOX1 variants were not determined.

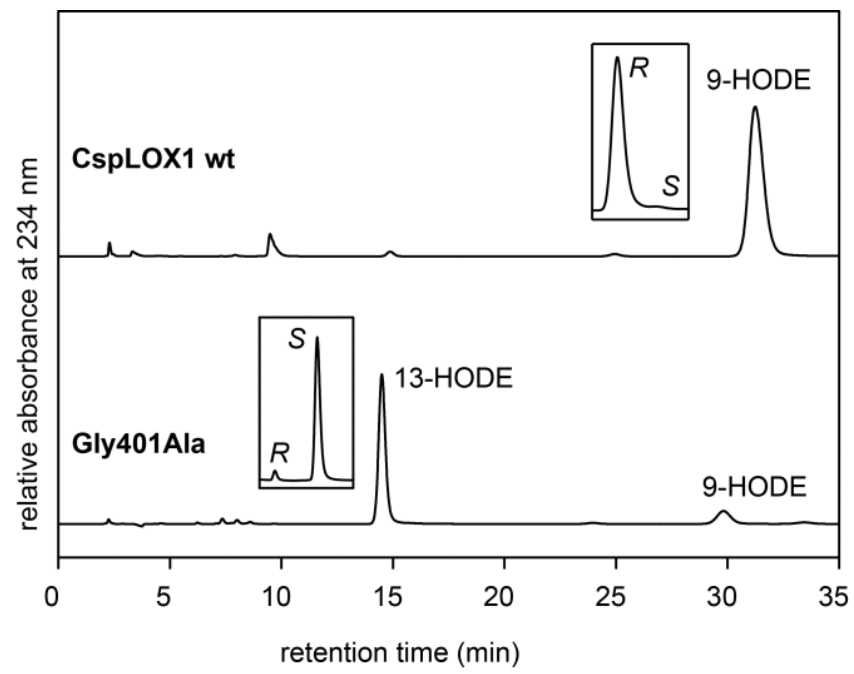

Figure 45. Products formed from linoleic acid by the Gly401Ala variant compared to CspLOX1 wt. $15 \mu \mathrm{g}$ of the purified enzyme was incubated with $50 \mu \mathrm{g}$ of substrate. The hydroperoxy products were reduced to hydroxides and analyzed by SP-HPLC. Insets show the distribution of $R$ and $S$ enantiomers of 9-HODE and 13-HODE, respectively. 


\subsection{The putative oxygen channel of $8 R$-LOX is also present in CspLOX2}

In addition to steric shielding, it seemed possible that a specific oxygen channel may deliver oxygen directly to C11 on linoleic acid. In the coral 8R-LOX, a channel was found that most likely transports oxygen to the active site (Neau et al., 2014). This channel is connected to the active site close to the Gly/Ala switch (Gly427 in 8R-LOX) and was also identified in the structures of human 15-LOX-2 and porcine 12-LOX (Figure 46). The position of this putative oxygen channel is favorable for an antarafacial relationship of hydrogen abstraction and oxygen insertion. The hydrogen would be abstracted by the iron on one side of the channel, while the oxygen is delivered to the opposite side. In the $8 R$-LOX structure crystallized in complex with arachidonic acid, the channel is located close to the double bond between the central carbon of the pentadiene system and the $n-2$ position, which correspond to the region between $\mathrm{C} 9$ to $\mathrm{C} 11$ on linoleic acid.

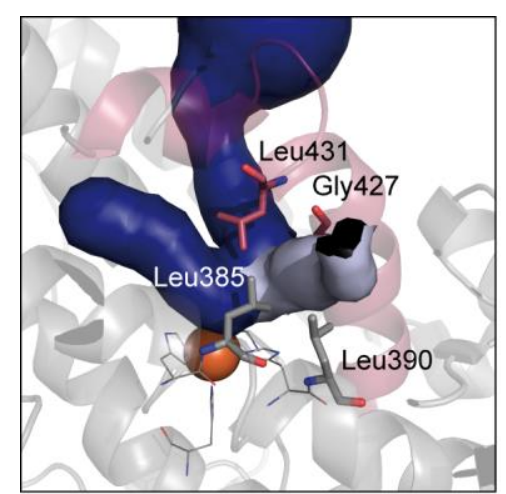

coral 8R-LOX (4QWT)

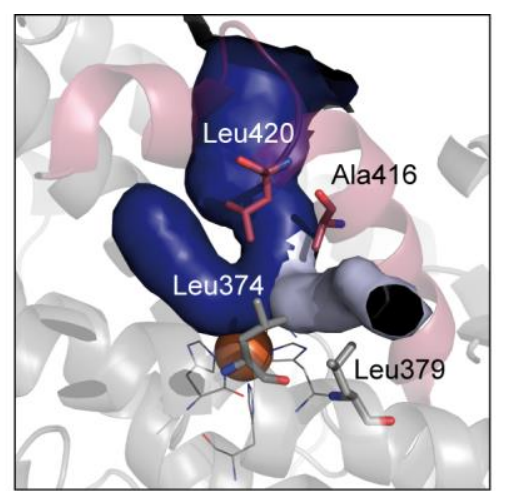

human 15-LOX-2 (4NRE)

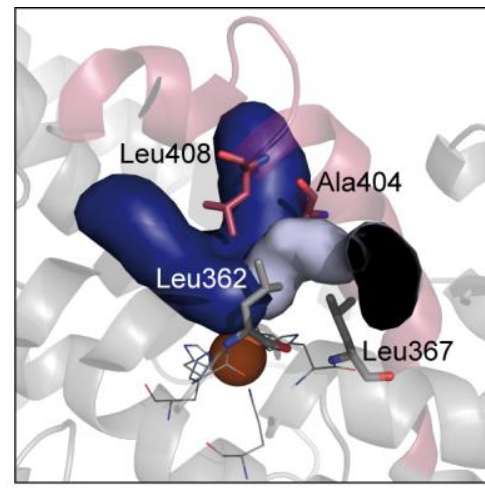

porcine 12-LOX (3RDE)

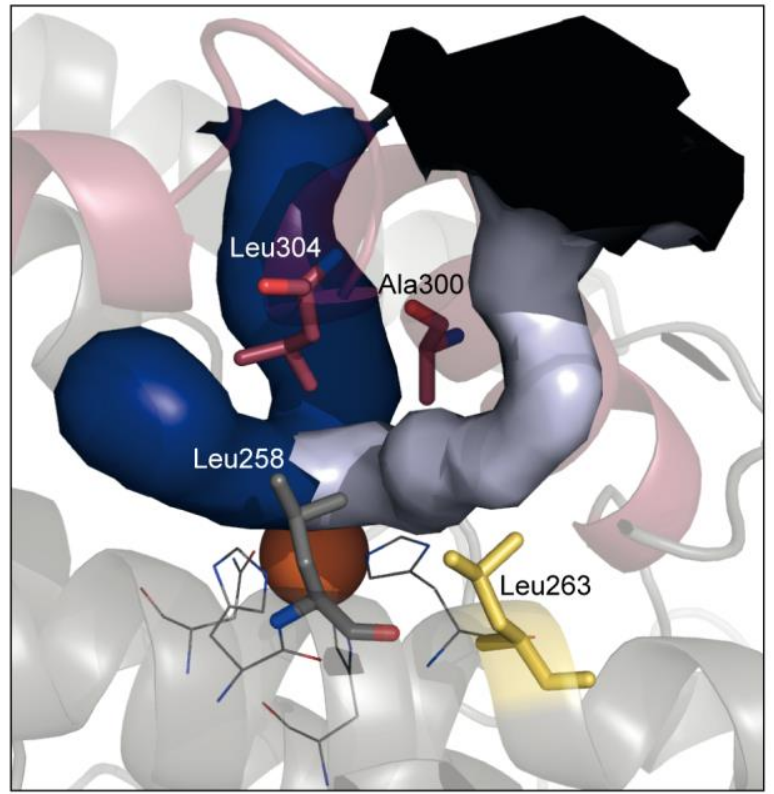

CspLOX2

Figure 46. Proposed oxygen channels in LOXs. Channels were visualized using the program Caver. The substrate binding channels are shown in dark blue, and the putative oxygen channel in light blue. The position of the arched helix is shown in dark red. The junction of both channels is surrounded by three invariant leucines (Leu385, 390, 431 in $8 R$-LOX) and the residue of the Gly/Ala switch (Gly427 in 8R-LOX). This channel is also found in CspLOX2. Here, Leu263 (yellow) corresponds to the residue located at the putative oxygen channel. 
Using the plugin Caver for PYMOL, tunnels were also visualized in CspLOX2. In addition to the supposed substrate binding channel, a second channel was predicted at the same position as the putative oxygen channel in $8 R$-LOX (Figure 46). This channel is surrounded by the amino acid residues Leu258, Leu263, Ala300 and Leu304 at the connection to the substrate binding channel. Of these residues, Leu258, Ala300 and Leu304 belong to the identified active site clamp described before (Chapter 3.4.2). Leu263 is slightly more distant from the active site and lines the putative oxygen channel with its side chain. Therefore, it could be possible to block this potential oxygen channel by increasing the size of this side chain. The enzyme variant Leu263Trp could thus provide valuable information about the existence of this oxygen channel and was tested for its catalytic activity and oxygenation specificity.

The mutation caused only a slight shift towards 13-HPODE and a reduction of enzymatic activity compared to the wt (Figure 47A). If this decline in activity is, however, attributed to a reduced oxygen accessibility remains unclear. To get an idea if the oxygen access is influenced by the mutation, the kinetic constants with limiting oxygen concentrations under fatty acid saturation were determined. The idea was that $K_{M}$ values sometimes indicate how well a substrate can bind to the active site of the enzyme.
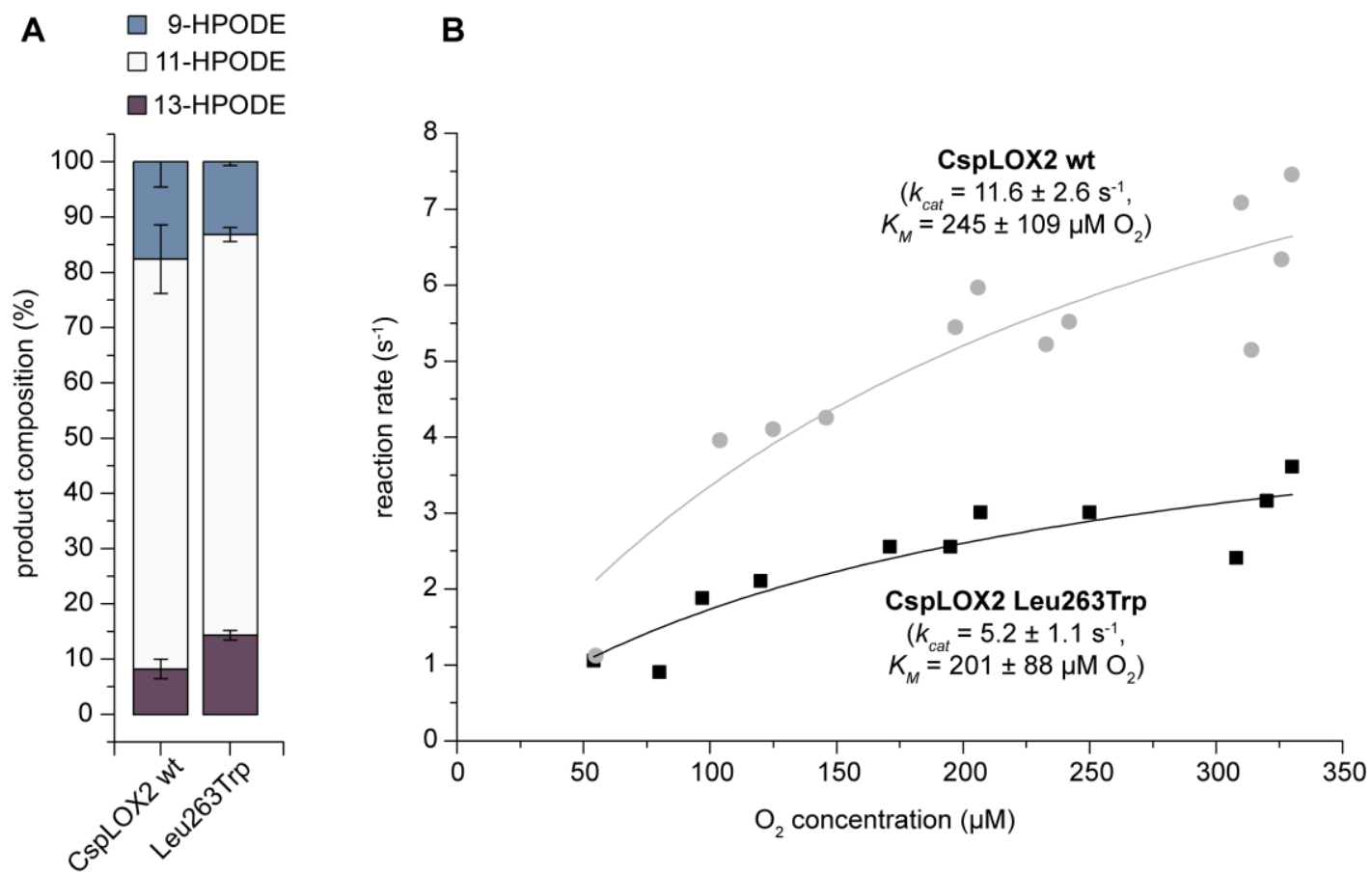

Figure 47. Oxygenation specificity and kinetics of CspLOX2 and its Leu263Trp variant for different oxygen concentrations. (A) The product composition formed by the enzyme variants was evaluated by HPLC analysis. The scaling factor was included in the quantification (Supplemental Figure 2). Shown are the mean values of 3 independent experiments with standard deviations for the column below. (B) Purified enzymes were incubated with $150 \mu \mathrm{M}$ linoleic acid in an oxygen electrode. Different oxygen concentrations were adjusted by flushing the substrate solution with $\mathrm{N}_{2}$ before adding the enzyme. The data were fitted to the Michaelis-Menten equation to determine $k_{\text {cat }}$ and $K_{M}$. 
Therefore, different oxygen concentrations were adjusted inside the oxygen electrode. To decrease the oxygen concentration, the buffer was flushed with nitrogen gas, to increase the oxygen concentration, the buffer was vigorously mixed with air. The $k_{\text {cat }}$ value of the Leu263Trp variant was about $50 \%$ of the wt activity $\left(5.2 \mathrm{~s}^{-1}\right.$ compared to $\left.11.6 \mathrm{~s}^{-1}\right)$. Furthermore, a $K_{M}$ value of $245 \pm 109 \mu \mathrm{M} \mathrm{O}_{2}$ for the wt enzyme and $201 \pm 88 \mu \mathrm{M} \mathrm{O}_{2}$ for the Leu263Trp variant were determined by fitting of the measured values to the Michaelis-Menten equation (Figure 47).

The differences of the $K_{M}$ values between wt and Leu263Trp variant are not significant. It should also be noted that the oxygen concentration at $30^{\circ} \mathrm{C}$ is saturated at approximately $230 \mu \mathrm{M}$ oxygen under a standard pressure of $101.1 \mathrm{kPa}$ (Murray and Riley, 1969), indicating that only about the half maximal velocity of both wt and Leu263Trp variant were reached at saturated oxygen levels. Therefore, an oxygen transport function of this channel cannot be deduced from these results.

\subsubsection{Xenon derivatization of CspLOX2 was not successful}

A more direct possibility to study the paths of oxygen in enzymes is by xenon derivatization of protein crystals. This gas resembles dioxygen in size and chemical properties and has sufficient electrons to be visualized in electron density maps. In other proteins, including copper amine oxidase (Pirrat et al., 2008), laccase (Kallio et al., 2011) or cytochrome ba 3 oxidase (Luna et al., 2012), sites of oxygen binding in the active site could be successfully identified. Therefore, crystals of CspLOX2 were prepared and derivatized in a pressurization cell. Different conditions were tested in the xenon chamber (pressure, incubation time, velocity of pressure release). Although crystals seemed to retain their shape and integrity after 100 Psi of xenon for 5 min with a slow pressure release during $30 \mathrm{~s}$, crystals did not diffract well when tested later at a synchrotron beam source. Therefore, it was not possible to identify oxygen channels in CspLOX2 with this method and experimental conditions. 


\subsection{No suitable hydrogen atom donors are found in the CspLOX2 active site}

One mechanistic model to explain the oxygenation specificity of LOXs focuses on the last step of the catalytic cycle. During this step, the peroxyl radical is trapped by a hydrogen atom, thereby preventing the reversible off reaction ( $\beta$-fragmentation) (Schneider et al., 2007). The CspLOX2 active site was hence analyzed for residues that might be good hydrogen atom donors. Such hydrogen atom donors may for example include tyrosine (van Overveld et al., 2000), tryptophan (Li et al., 1991) or cysteine (Giles et al., 2003) side chains. To estimate the location of relevant residues relative to the reacting pentadiene, the substrate crystallized in $8 R$-LOX was again chosen as model (Neau et al., 2014) (Figure 48 and Figure 49).

In the environment of the active site, i.e. within a $10 \AA$ radius around the pentadiene, no tryptophan and only one cysteine residue was found. The side chain of Cys507 is however located in a $10.1 \AA$ distance from the middle position of the reacting pentadiene group. Additionally, it is facing the pro-S side of the bis-allylic carbon. The $11 R$-peroxyl radical which leads to $11 R$-HPODE formation is, however, located on the opposite site of the linoleic acid molecule. A catalytic function of this cysteine residue thus seemed unlikely.

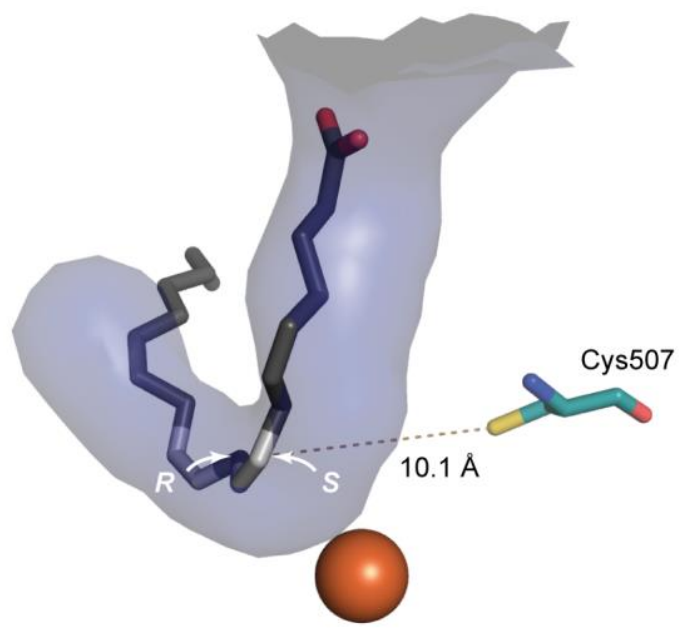

Figure 48. Position of Cys507 relative to the substrate binding channel. The arachidonate ligand of 8R-LOX (Neau et al., 2014) was used to estimate the position of the pentadiene in the active site. Only one cysteine residue was identified in a $10 \AA$ radius around the middle position of this pentadiene. Nevertheless, the thiol group of Cys507 (green) is located rather far away from this pentadiene and faces the pro-S side of the bis-allylic position.

Tyrosines were neither in an optimal position to trap peroxyl radicals on the substrate (Figure 49A). They are too far away and their side chains are not oriented towards the substrate. But if Tyr310 would adopt an alternative conformation during catalysis, it might get close enough to trap the bis-allylic hydroperoxide. To test this hypothesis, Tyr310 was replaced by isoleucine or phenylalanine. These mutations had only a very little effect on the activity and specificity of the enzyme (Figure 49B), suggesting that peroxyl trapping by Tyr310 plays no role for the formation of 11-HPODE. 

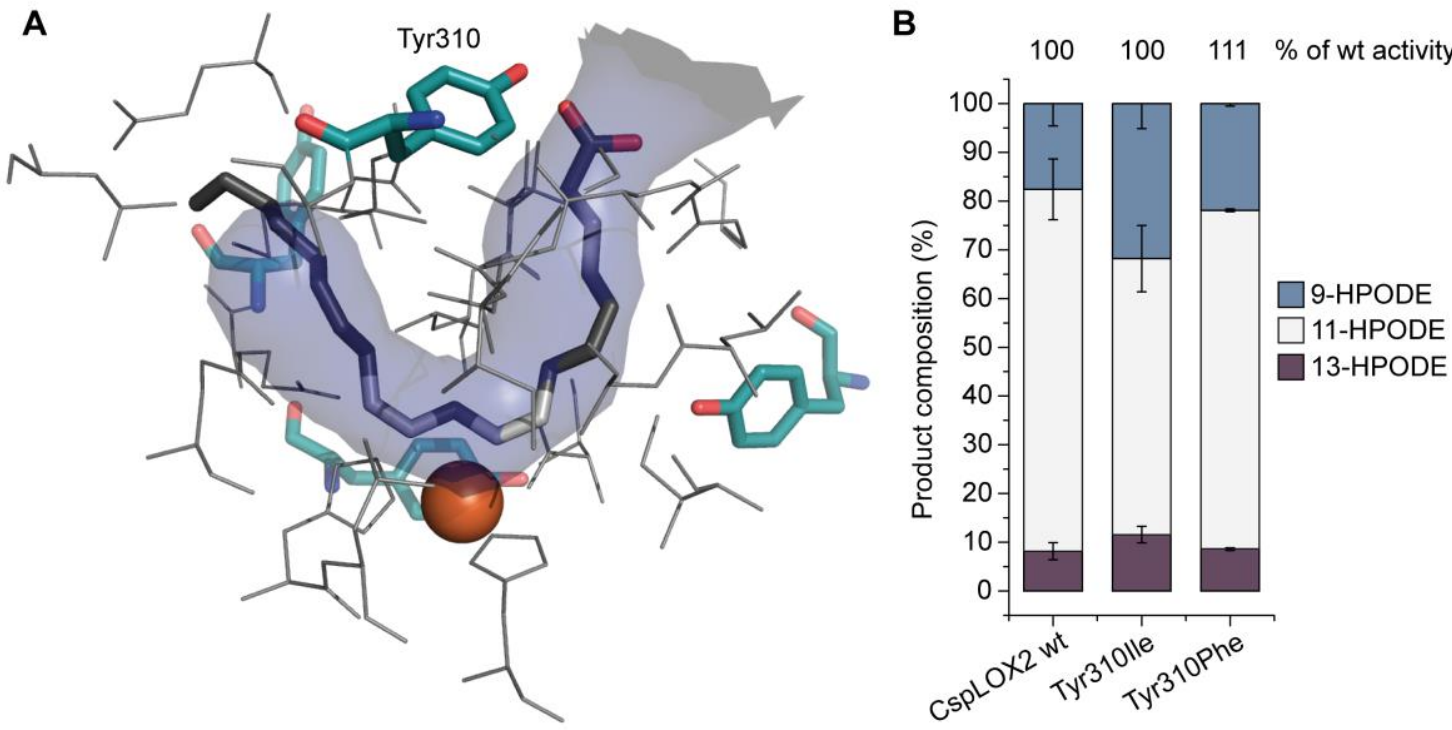

Figure 49. Influence of Tyr310 on the oxygenation specificity. (A) The side chain of Tyr310 is oriented away from the pentadiene system but might move during catalysis. Other tyrosines in proximity of the active site are shown as green sticks. The arachidonate ligand of the $8 R$-LOX is shown as black sticks with the double bonds of the reacting pentadiene in gray. The catalytic iron is shown as orange sphere. (B) The oxygenation specificity and catalytic activity was not affected by the Tyr310lle or Tyr310Phe exchange. The peroxidation products of linoleic acid were subjected to SP-HPLC analysis. The scaling factor was used for the quantification. Shown are the mean values of 3 independent experiments with standard deviations for the column below. Additionally, the relative catalytic activities indicated above the columns were determined using the oxygen electrode. 


\subsection{Computational approach supports role of steric shielding for 11-HPODE} formation

Due to the confined space in the CspLOX2 active site, it seemed possible that the pentadiene has to adopt a slightly twisted conformation that would result in an increased spin density on C11 and thus in a preferred oxygen insertion at this position. To get an insight if this hypothesis plays indeed a role, a computational analysis was carried out by Martin Werner and Prof. Dr. Ricardo Mata (Institute of Physical Chemistry, University of Göttingen, Germany). The singly occupied molecular orbital (SOMO) spin densities were determined for different dihedral angles $\varphi$ (Figure 50A and C). This reflects the likelihood of the radical to be found at one of the positions C9, C11 or C13 on linoleic acid. Although a torsion of the otherwise planar pentadiene system of up to $30^{\circ}$ resulted in a slight shift of SOMO spin density (Figure $50 \mathrm{C}$ ), the product compositions of CspLOX2 variants cannot exclusively be explained with a localization of spin density. Furthermore, the spin density distribution would suggest that even in a planar system, the reaction would preferentially occur at the middle position, since this is the position with the highest spin density. It is known from free radical chemistry that this is not the case (Yin and Porter, 2005).

A

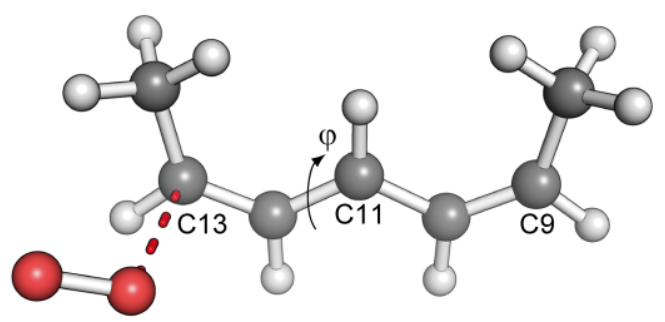

C

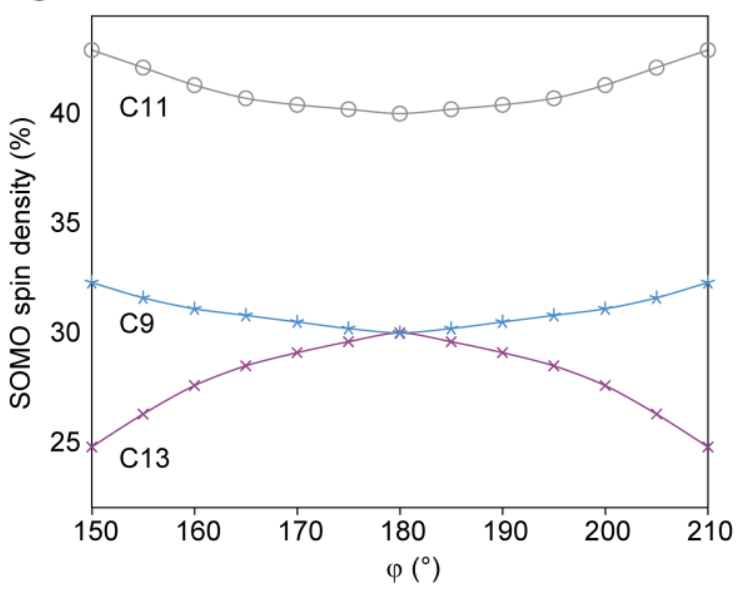

B

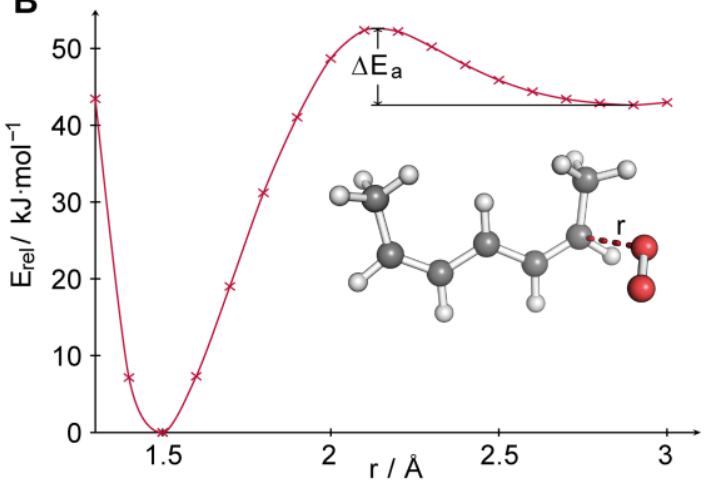

D

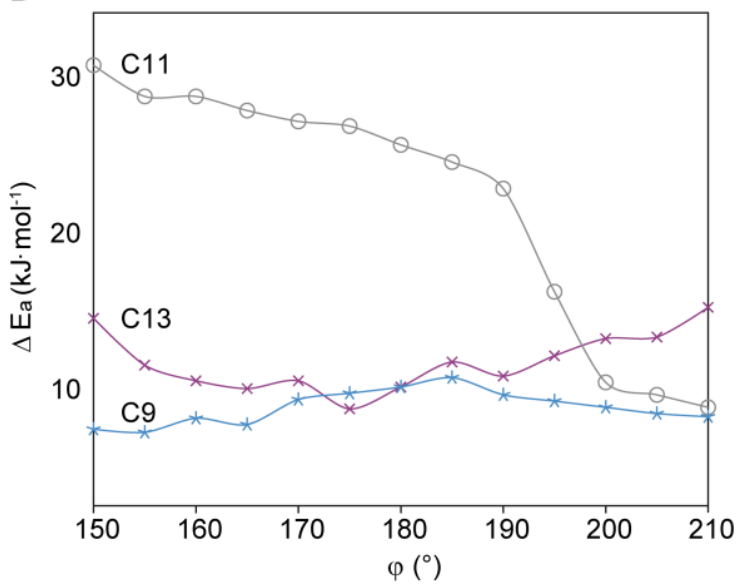

Figure 50. Spin density distribution and activation barrier on C9, C11 and C13 of linoleic acid in dependence of the dihedral angle $\varphi$. (A) A 2,5-heptadiene system was used as model system resembling carbon atoms 8-14 on linoleic acid. The dihedral angle $\varphi$ is indicated as well as the oxygen molecule (red). (B) When oxygen is inserted, an activation barrier has to be overcome. (C) The SOMO spin density is shown for different angles $\varphi$. C9, C11 and C13 correspond to the three positions indicated in panel A. (D) Oxygen was narrowed to the 2,5-heptadiene system in relaxed surface scans using B3LYP-D3/def2-TZVP and the activation energy was determined for different angles $\varphi$. 
Therefore, Martin Werner further analyzed the reactivity at the positions C9, C11 and C13 of linoleic acid. The radical formed in 2,5-heptadiene was chosen as model system and oxygen was narrowed in relaxed surface scans using B3LYP-D3/def2-TZVP (Figure 50B). During oxygen addition, an activation barrier $\left(\triangle \mathrm{E}_{\mathrm{a}}\right.$, Figure $\left.50 \mathrm{~B}\right)$ has to be overcome. These activation barriers were determined for the different angles as shown in Figure 50D. When the system is planar at a dihedral angle of $180^{\circ}$, no reaction will occur on C11 due to a high activation barrier. In contrast, when the dihedral angle is increased to $200^{\circ}$, this reaction path becomes competitive to $\mathrm{C} 9$ and C13 (Figure 50C).

While this model can explain how the activation barrier on C11 can be lowered to allow oxygen addition at the bis-allylic position, the observed product distribution for the CspLOX2 variants cannot be explained. Therefore, the idea that the distribution might be a result of steric shielding was addressed through a theoretical approach using MD simulations by our collaboration partners. To assess which role steric shielding might play, the environment of the linoleic acid substrate in the CspLOX2 active site was analyzed in a number of simulations and snapshots. For this analysis, an equalization plane was defined in the pentadiene system (Figure 51A). From all three carbon atoms that are involved (C9, $C 11$ and $C 13$ ), a normal (Figure 51A, white dotted axis) is projected to the antarafacial side (towards $9 R, 11 R, 13 S$ ) of the linoleic acid molecule and a point is found in $3 \AA$ distance from the plane. If there is no atom closer than $2.2 \AA$ to this point (Figure 51A, brown transparent spheres), the carbon site is fully accessible. On the other hand, if any atom is closer than $1.8 \AA$ to this point, the site is fully shielded. For distances in between, an exponential function describes the decline in reactivity of the carbon site. That means if one atom gets too close to the carbon site (Figure 51A, red atoms), the position is defined as less reactive. In the example shown in Figure 51A, $C 9$ and $C 13$ are thus considered to be less reactive and oxygen is rather inserted at C11. Evaluation of a number of simulations and snap shots results in a relative distribution of reactive sites. These distributions were analyzed for the wt enzyme as well as for all variants on the antarafacial side, comprising Leu258Val, Ala300Gly, Leu304Val and Leu502Val. As the activation barrier is in general higher on C11 (Figure 50D), the relative amount of reactions on C11 was lowered by $10 \%$ and distributed equally to C9 and C13. A clear correlation of these simulated reactive sites with the experimentally obtained product distributions could be observed (Figure 51B), supporting the hypothesis that steric shielding plays indeed an important role for controlling the oxygenation specificity.

Notably, the four analyzed protein variants carry mutations on same side as the proposed oxygen channel and opposite the iron (facing $9 R, 11 R, 13 S$ ) (Figure 51C), which is the intuitive side for oxygen insertion and was thus selected for this analysis (Figure 51A). This model is, however, unable to explain how the stereospecificity could be inverted in some other variants carrying mutations on the same side as the iron (facing 9S, 11S, 13R) like lle296Ala or Leu506Val (Figure 51C). 
A

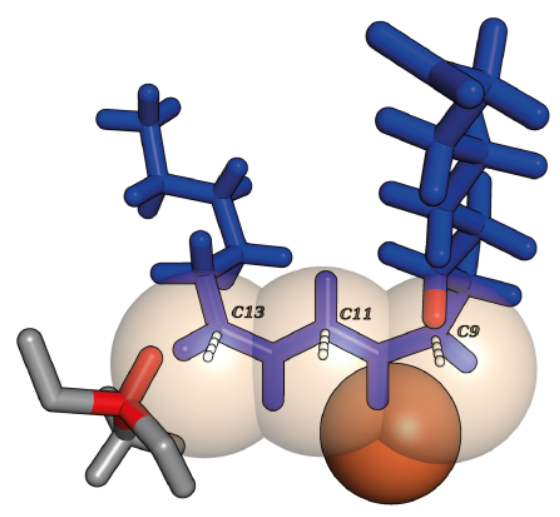

B

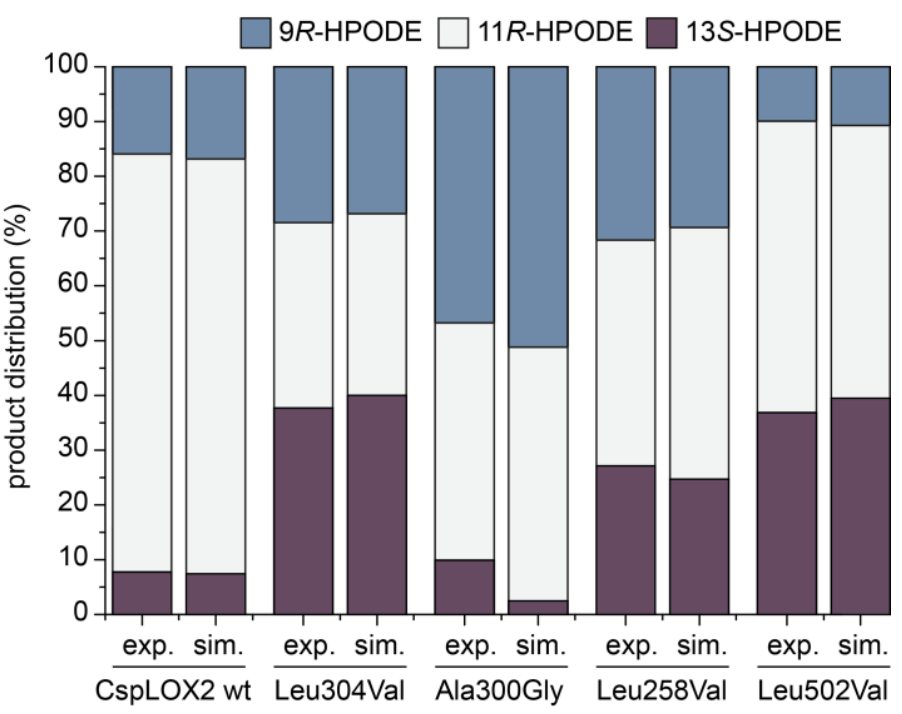

C

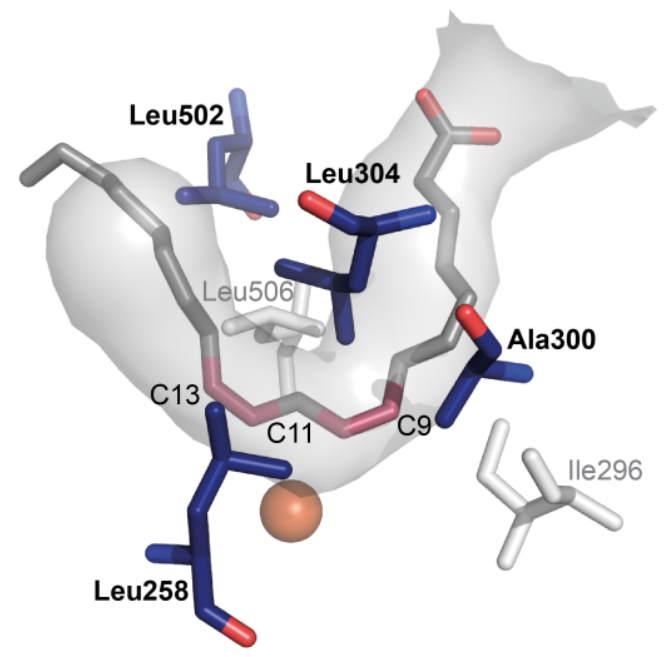

Figure 51. Simulation of reactive positions on the linoleic acid substrate. (A) The model of linoleic acid (blue) in the active site of CspLOX2 was used for the simulations. Analyzed were positions 9,11 and 13 on the opposite side of the iron (towards $9 R, 11 R, 13 S$ ). A plane is constructed in the pentadiene system and normals on this plane through C9, C11 and C13 are shown as dashed white lines. In a distance of $3 \AA$ a point is defined. The reactivity of the carbon site is higher if the space around this point (brown transparent spheres) is free. In this model, C9 and C13 would be less reactive as the red atoms get too close. (B) From several simulations and snapshots obtained with CspLOX2 wt, Leu258Ala, Ala300Gly, Leu304Val and Leu502Val, a distribution of reactive sites on $9 R, 11 R$ and $13 S$ was determined (sim.). This distribution correlates with the experimentally observed distribution of the respective HPODEs (exp.) formed by each of the variants. (C) For this analysis, only mutations on the antarafacial side of the substrate (residues shown in blue) were considered. Iron is shown as orange sphere, the substrate binding channel of CspLOX2 in gray and the substrate crystallized in complex with $8 R$-LOX as grey sticks with the double bonds of the reacting pentadiene in dark red. The numbering corresponds to the pentadiene of linoleic acid. 


\section{Discussion}

In this study, two LOXs from Cyanothece PCC8801 were structurally and functionally characterized. Both enzymes, CspLOX1 and CspLOX2, revealed interesting features to study the domains required for membrane binding and the molecular basis of the oxygenation specificity. While the structure of the catalytic domain is highly conserved, different domains are found at the N-terminus of LOXs. This structural diversity of the $\mathrm{N}$-terminal domain and its potential function for substrate acquisition will be discussed in the first part of this section. Furthermore, the major aim of this study was to identify factors that are involved in the formation of the bisallylic product by CspLOX2. As CspLOX2 produces the highest relative amount of 11-HPODE from linoleic acid observed so far, the enzyme represented an ideal candidate for studying the underlying mechanism of this specificity. Of all possible factors, special focus was on the role of the metal cofactor, oxygen accessibility, selective peroxyl radical trapping and torsion of the pentadiene. To which extend these aspects contribute to the CspLOX2 specificity will be discussed here. Furthermore, it will be evaluated whether these findings may also apply to MnLOXs and even to classical LOXs that do not produce bis-allylic products.

\subsection{The N-termimal helices of CspLOX1 may be involved in substrate acquisition from membranes}

So far, only few bacterial LOXs have been characterized including several from cyanobacteria and one from P. aeruginosa (Andreou et al., 2008; Garreta et al., 2013; Hansen et al., 2013; Lang et al., 2008; Senger et al., 2005; Zheng et al., 2008). Of these, only the enzyme from $P$. aeruginosa has been crystallized (Garreta et al., 2013). Thus, the two structures from cyanobacterial enzymes described here considerably extend the knowledge about bacterial LOXs and help understand the structural diversity of LOXs. While classical LOXs consist of a conserved two domain structure with a $\beta$-barrel domain at the $\mathrm{N}$-terminus and an $\alpha$-helical LOX domain at the $\mathrm{C}$-terminus, both LOXs from Cyanothece sp. revealed unusual characteristics within the $\mathrm{N}$-terminal domain (Figure 16). While CspLOX2 lacks the $\beta$-barrel domain completely, CspLOX1 contains not only the $\beta$-barrel domain, but in addition an $\alpha$-helical extension. So-called mini-LOXs like CspLOX2 consisting only of the catalytic domain are not unusual among bacterial LOXs (An et al., 2015; Koeduka et al., 2007). The fact that the $\beta$-barrel domain is dispensable for LOX catalysis was also found by truncation of classical two-domain LOXs that retained their catalytic activity (Di Venere et al., 2003; Maccarrone et al., 2001; Walther et al., 2002; Walther et al., 2011). Other bacterial and coral LOXs were found to express fusion proteins that contain a second domain with an additional catalytic activity at the $\mathrm{N}$-terminus. The cyanobacterium Acaryochloris marina for instance expresses a fusion protein with a catalase-like domain at the $\mathrm{N}$-terminus (Gao et al., 2010) and also the corals P. homomalla and G. fruticosa carry a hemoprotein at the $\mathrm{N}$-terminus with allene oxide synthase activity (Boutaud and Brash, 1999; Lõhelaid et al., 2008). In addition, bacterial LOXs can also harbor additional $\alpha$-helices instead of 
a $\beta$-barrel domain as observed in the crystal structure of the $P$. aeruginosa LOX (Garreta et al., 2013). The $\mathrm{N}$-terminal domain of CspLOX1, however, contains a $\beta$-barrel domain as found in classical LOXs and in addition the $\alpha$-helical extension reminiscent of the helical insertion found in the $P$. aeruginosa LOX (Figure 16). Although a $\beta$-barrel domain is present in CspLOX1, it exhibits one remarkably elongated $\beta$-sheet and an altered orientation compared to other LOXs (Chapter 3.1.3). This could reflect a structural flexibility which was also observed by small angle $X$-ray scattering experiments for the rabbit 15-LOX. Here, the movement of the $\beta$-barrel relative to the catalytic domain resulted in a similar conformation (Hammel et al., 2004). It would be conceivable that the $\beta$-barrel of CspLOX1 has a rotational flexibility similar to the one of the rabbit 15-LOX, but that another conformation has been conserved in the crystal structure. Alternatively, the interaction of the helical extension and the $\beta$-barrel domain with the catalytic domain might also restrict the structural flexibility.

The additional $\alpha$-helical extension is fused to the $\mathrm{N}$-terminus of the $\beta$-barrel domain by a short linker region. The two amphipathic helices interact via mainly polar contacts with a long helix of the catalytic domain, while covering the exposed hydrophobic side with hydrophobic residues of the same helices contributed by a neighboring molecule in the crystal lattice. It is likely, that the hydrophobic side of the amphipathic helices serves another function in solution, for example binding to membranes. Flotation experiments with liposomes consisting of phosphatidylcholine suggested that the helical extension is crucial for membrane binding since truncation of this domain substantially impaired binding to the vesicles (Figure 22). The $\beta$-barrel domain alone was not sufficient to fulfill this function.

Membrane binding has so far been mainly attributed to the $\beta$-barrel domain. Three tryptophans that have been shown to be crucial for membrane binding in 5-LOX (Kulkarni et al., 2002) are not conserved in CspLOX1. Moreover, the membrane binding function of the $\beta$-barrel domain is assumed to be mediated by $\mathrm{Ca}^{2+}$ which binds to sites within loop regions of the $\beta$-barrel domain and interacts with the membrane, as typical for $\mathrm{C} 2$ domains (Oldham et al., 2005; Tatulian et al., 1998). Although it seems unlikely, it cannot be ruled out from the experimental conditions that the $\beta$-barrel domain of CspLOX1 contributes to membrane binding under high $\mathrm{Ca}^{2+}$ concentrations. Studies on the rabbit 15-LOX also showed that truncation of the $\beta$-barrel domain impaired the membrane binding capacity, but did not abolish it completely, which was proposed to be related to hydrophobic residues of the catalytic domain that function in membrane binding (Walther et al., 2002). However, truncation of covering domains might also expose the hydrophobic core of the enzyme which may result in an artificial binding to membranes. 

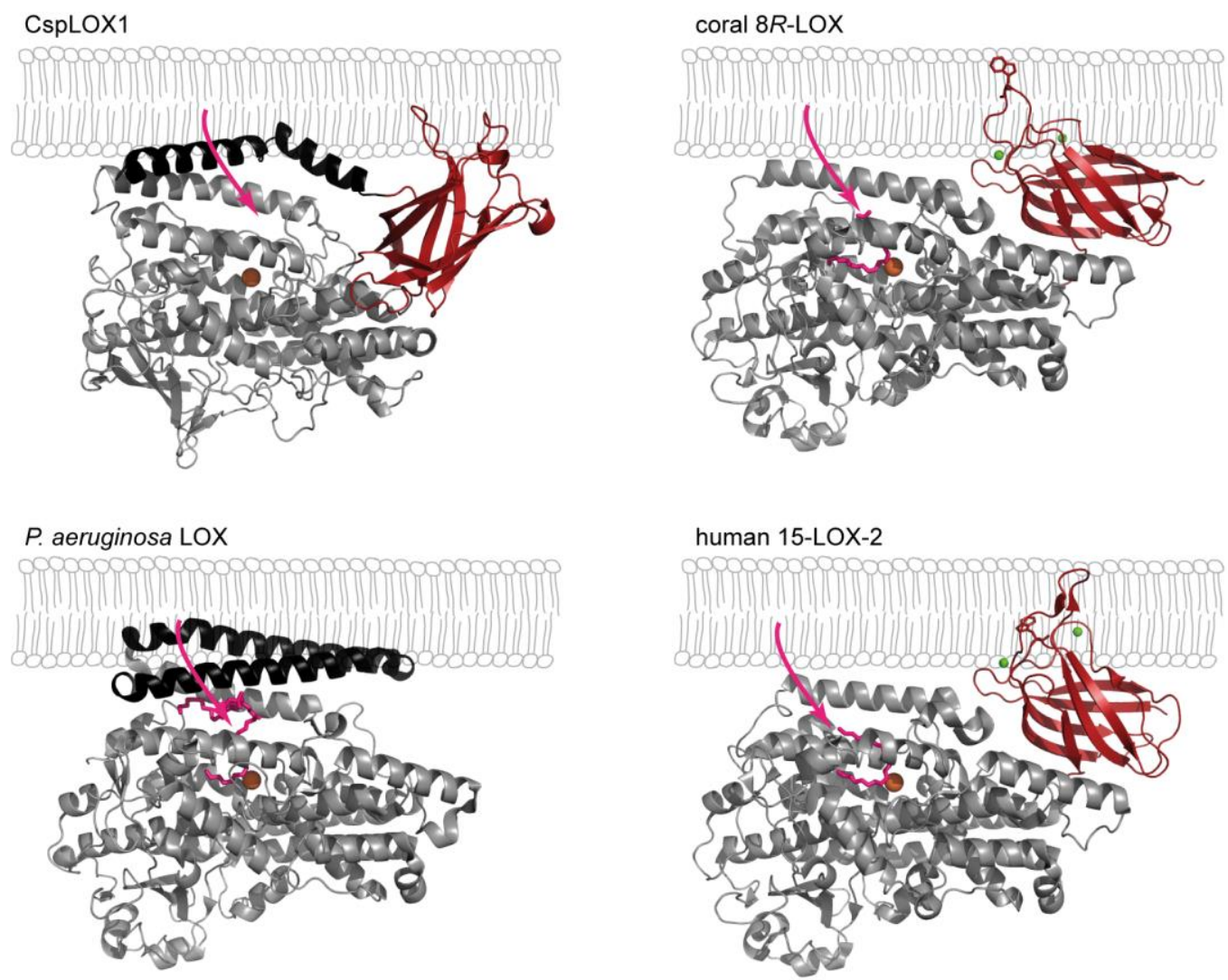

CspLOX2
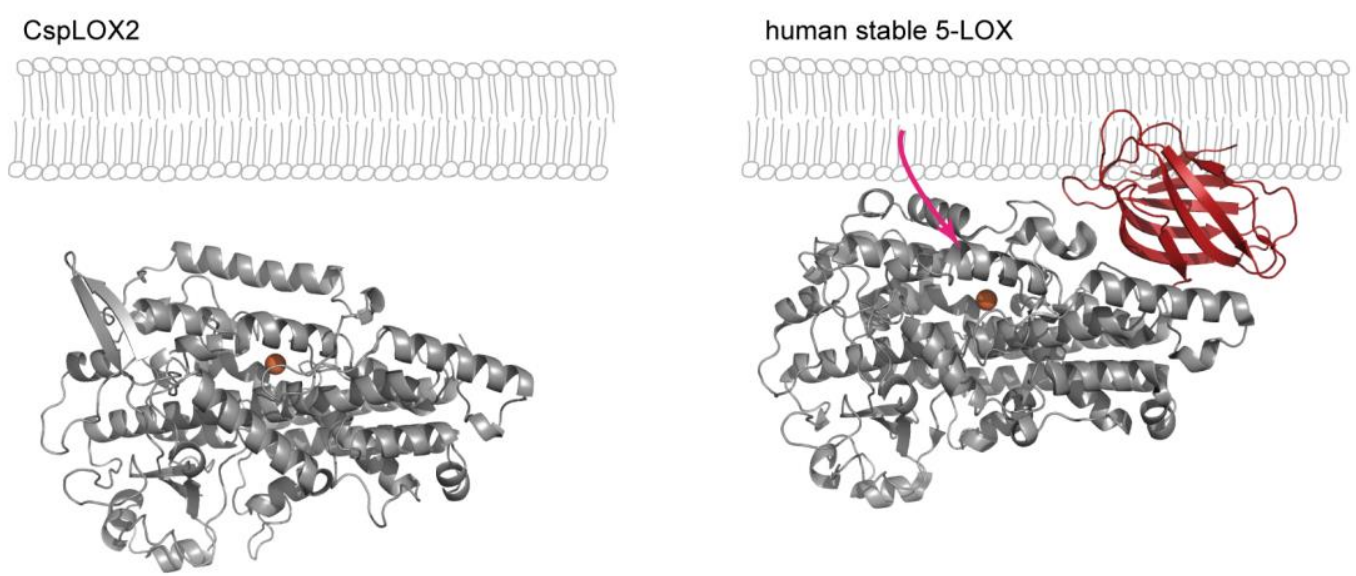

Figure 52. Model of membrane binding and substrate acquisition in different LOXs. The catalytic domains of the enzymes are shown in gray, the $\beta$-barrel domain in red, the helical insertion/extension in black and the iron as orange sphere. The substrates and substrate analogs bound to the active site are shown as pink sticks. The entrance to the substrate binding channel is indicated by a pink arrow. Binding via the helical insertion/extension or the $\beta$-barrel domain would orient the entrance to the substrate binding channel towards the membrane which is shown as schematic bilayer. 
As the $\beta$-barrel domain of CspLOX1 could not significantly contribute to the membrane binding ability of the enzyme, it is likely that only the amphipathic helices mediate this function. Amphipathic helices that function in membrane binding have also been found in other proteins, like the yeast mitochondrial protein TIM44 which binds with two amphipathic helices to the periphery of mitochondrial membranes (Cui et al., 2011). It is also possible that the long helical insertion in the $P$. aeruginosa LOX mediates membrane binding. These helices, which also have an amphipathic character, even interact with the phospholipid bound to the active site of this enzyme (Garreta et al., 2013). As phospholipids present the major building block of most biomembranes, this may also suggest how substrates are directly acquired from the membrane. Interestingly, the entrance to the substrate binding channel would be oriented towards the membrane if the helices of the $P$. aeruginosa LOX or CspLOX1 mediate membrane binding (Figure 52). This orientation towards the membrane would also be achieved when enzymes bind to the membrane via the $\beta$-barrel. Recently, it was also reported that the $P$. aeruginosa LOX directly acts on membranes and induces hemolysis in red blood cells (Banthiya et al., 2015). As no further hormones or signaling molecules like jasmonic acid, leucotrienes or resolvines were found in cyanobacteria and other prokaryotes, it seems likely that one of the first function of LOXs was directly on membranes, thereby altering the membrane composition and chemical properties, rather than producing signaling molecules.

Later in evolution, more versatile pathways might have evolved that require a cascade of different enzymes, like allene oxide synthase, hydroperoxide lyase or divinyl ether synthase in plants (Andreou et al., 2009) and prostaglandin synthase, thromboxane synthase or leukotriene hydrolase in animals (Kühn et al., 2015; Shimizu, 2009). These pathways which also may require a liberation of free fatty acids by phospholipases from the membrane then produce a diversity of biomediators that transfer a signal from one cell to the other. These pathways are probably not necessary in single cell organisms like prokaryotes. It is nonetheless fascinating, why only a very low amount of approximately $0.5 \%$ of all sequenced bacterial genomes $(13,000)$ contain LOX genes, while the vast majority does not (Horn et al., 2015). Few bacteria seem to have an advantage from the LOX genes since they remain in their genomes and are further evolved. In other bacteria they are disrupted, suggesting that they could not fulfill a useful function. Despite some hints about the function of the $P$. aeruginosa LOX, almost nothing is known about the biological role of LOXs in other bacteria. It would thus be interesting to understand which physiological role these enzymes might have in cyanobacteria. Since a number of LOX genes were found in nitrogen fixing cyanobacteria as well as nitrifying bacteria, and since LOXs may also act as nitrogen storage protein in soybean (Grimes et al., 1993; Tranbarger et al., 1991), a possible role in the nitrogen metabolism has been suggested (Koeduka et al., 2007). Direct evidence for this hypothesis has, however, never been reported. And though a direct role in membrane modification is a likely function of bacterial LOXs, this might not be true for all of them. The mini-LOX CspLOX2 for example did not bind to liposomes in the flotation experiment (Figure 22), suggesting that it does not act directly on membranes. 
As CspLOX1 exhibits only a very low sequence homology to other LOXs, it was placed as outgroup in a phylogenetic tree. Such outgroups allow to define a root of the tree and thus a better reconstruction of evolutionary relationships (Graham et al., 2002). From the obtained phylogenetic tree, several characteristics of a common LOX ancestor can be concluded: First of all, it probably consisted of a $\beta$-barrel domain and an $\alpha$-helical domain as these two domains can be found in CspLOX1 as well as in human, coral and plant LOXs (Figure 15). The mini-LOXs found among bacterial enzymes probably emerged from a loss of the $\mathrm{N}$-terminal domain. In other cases, a second catalytic domain with another activity like allene oxide synthase was fused to the N-terminus of the catalytic domain (Boutaud and Brash, 1999; Lõhelaid et al., 2008) or long additional helices as found in CspLOX1 or the 15-LOX from P. aeruginosa (Garreta et al., 2013) became part of the structure and may now function in membrane binding. It is therefore obvious, that the N-terminal domains are dispensable for LOX catalysis but probably have auxiliary functions.

Furthermore, ancient LOXs probably contained iron in the active site that was coordinated by three histidines, an asparagine and a C-terminal isoleucine. In case of some fungal enzymes, manganese was employed as catalytic metal which might be an adaptation to the low availability of iron in some natural environments or as protection to oxidative stress (Anjem et al., 2009; Imlay, 2008; Sobota and Imlay, 2011). This has also been suggested for other enzymes that are known to contain either manganese or iron as catalytic metal, such as superoxide dismutase (SOD) (Lah et al., 1995) or ribonucleotide reductases (Martin and Imlay, 2011). Some of the MnLOXs also contain valine instead of isoleucine at the $\mathrm{N}$-terminus. In some mammalian enzymes, a fourth histidine or a serine is found at this position (Figure 15 and Supplemental Figure 4). These alterations probably developed later in evolution.

Regarding the reaction of LOXs, it is likely that the substrate initially bound into the active site in a tail-first orientation, resulting in $13 S$ - and $9 R$-hydroperoxides from linoleic acid and $11 R$ - and $15 S$-hydroperoxides from arachidonic acid. Both specificities are interchangeable by a single amino acid exchange of the Gly/Ala switch which is also conserved in such a phylogenetically distant enzyme like CspLOX1. The reversed orientation that was found in some plant and mammalian enzymes (Egmond et al., 1972; Hornung et al., 1999; Schwarz et al., 1998) probably emerged later in evolution. This is most likely also true for the unusual bis-allylic specificity of CspLOX2 and MnLOXs. 


\subsection{Although 11-HPODE is formed and isomerized in a very similar way in MnLOXs and CspLOX2, the catalytic metal is not interchangeable}

The unusual specificity for bis-allylic products was so far only shown for fungal MnLOXs and the iron-containing CspLOX2. But while the reaction of the 13-MnLOX from the fungus G. graminis has been studied in great detail, the formation and isomerization of 11-HPODE by CspLOX2 left open questions. One of the major questions was if 11-HPODE is required as intermediate and 9and 13-HPODE are only formed by isomerization or if a mixture of HPODEs is formed and thereof only 11-HPODE is subsequently isomerized to the conjugated hydroperoxides (Figure 29). It was also important to understand in which time frames the peroxidation of linoleic acid and isomerization of 11-HPODE to the conjugated hydroperoxides occur. Knowledge about the individual steps and their products is a prerequisite to analyze suitable CspLOX2 variants which may reveal the underlying mechanisms of 11-HPODE formation. It also allows to identify parallels and differences of the CspLOX2 reaction with those of MnLOXs.

Analysis of the CspLOX2 reaction revealed that during a rather long lag phase the inactive Fe(II) in the metal binding site of CspLOX2 is converted to the active Fe(III) form, as suggested by EPR and kinetic analyses (Figure 27 and Figure 32). This oxidation of the iron cofactor, which has also been reported for SLOX1, is probably catalyzed by HPODEs which are the reaction products of the LOX reaction (Haining and Axelrod, 1958; Schilstra et al., 1992). A certain small amount of HPODE is probably always present in the assay, as free fatty acids can also be converted to the respective hydroperoxides by autoxidation (Figure 2 ). The activation process will then proceed exponentially in a chain reaction, as active LOX molecules produce more HPODE, thereby activating other LOX molecules. It should be noted that the kinetics of the enzyme activation may vary in different LOXs, depending for example on HPODE binding. This aspect was not addressed in this study, but might explain the different lengths of lag phases observed in different enzymes (Figure 25).

After activation, a mixture of 9-, 11- and 13-HPODE is produced by CspLOX2 simultaneously as shown by HPLC analysis (Figure 30). This model is further supported by kinetic data, since the isomerization of 11-HPODE to the conjugated HPODEs is too slow to explain the fast initial reaction rate (Table 7). When the substrate is used up, the first peroxidation reaction is finished and the remaining portion of 11-HPODE is subsequently isomerized to the conjugated products at a slower rate (Figure 25D). This transition from the peroxidation reaction to the isomerization reaction is reflected as kink in the progression curve obtained at $234 \mathrm{~nm}$ (Figure 53). These reaction phases are summarized in Figure 53. 


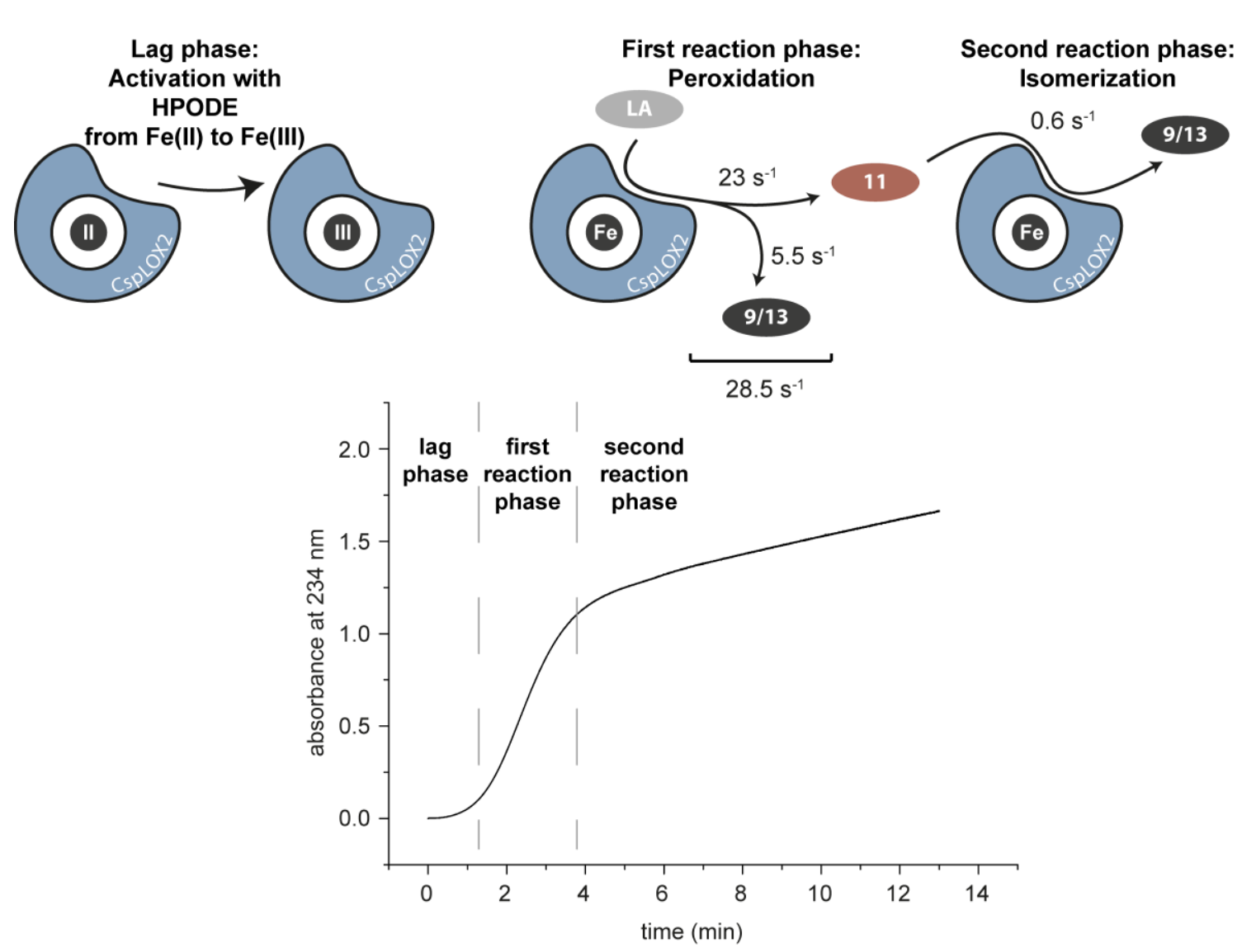

Figure 53. Explanation for the three reaction phases of CspLOX2. The lag phase is caused by the activation from Fe(II) to Fe(III). During the first reaction phase, linoleic acid (LA) is converted to a mixture of 9-, 11- and 13-HPODE and during the second reaction phase, 11-HPODE is isomerized to 9- and 13-HPODE at a slower rate.

Knowing that a mixture of 9-, 11- and 13-HPODE is formed during the first peroxidation reaction is important for the analysis of CspLOX2 variants. Determination of 11-HPODE formation should always be carried out after the peroxidation phase in order to obtain reproducible results. Furthermore, the results suggest that positions 9, 11, and 13 on linoleic acid are competing for oxygen insertion. The specificity of CspLOX2 is rather low compared to other LOXs which sometimes have specificities of more than $95 \%$ for a single regio- and stereospecific product like CspLOX1 that produces almost exclusively 9R-HPODE (Newie et al., 2015).

As kinetic and spectroscopic data are available for the 13-MnLOX of G. graminis (Hamberg et al., 1998; Oliw et al., 2004; Su et al., 2000), both types of enzymes with bis-allylic products and different metal cofactors can be compared. Relevant reaction features and kinetics of the individual phases are summarized in Table 11.

Despite the known facts that CspLOX2 contains iron instead of manganese and inserts oxygen in an antarafacial instead of a suprafacial way, interesting similarities could be identified. Most importantly, 11-HPODE is not required as intermediate, but instead a mixture of different HPODEs is formed during the first reaction phase. During the second phase, 11-HPODE is then isomerized to conjugated HPODEs (Hamberg et al., 1998) (Figure 53). The velocity of the 
peroxidation phase is similar in both enzymes, with $26 \mathrm{~s}^{-1}$ for MnLOX and $28 \mathrm{~s}^{-1}$ for CspLOX2. The fact that a biphasic reaction at $234 \mathrm{~nm}$ was observed with CspLOX2 but not with $13 R$-MnLOX is probably due to different kinetics of the 11-HPODE formation and isomerization. While CspLOX2 forms 11-HPODE at a $\sim 40$-fold higher rate than isomerizing it to the conjugated HPODEs $\left(23 \mathrm{~s}^{-1}\right.$ vs. $\left.0.6 \mathrm{~s}^{-1}\right)$, MnLOX forms 11-HPODE at a slower rate than isomerizing it $\left(7 \mathrm{~s}^{-1} \mathrm{vs} .9 \mathrm{~s}^{-1}\right)$ (Table 11). Consequently, the intermediate can accumulate in the CspLOX2 reaction, while it is surprising that it can be detected in the MnLOX reaction at all. The high rate of 11-HPODE formation is also reflected in the product composition after the first reaction phase. While $13 R-M n L O X$ produces only up to 20-30 \% of the bis-allylic product in addition to 70-80 \% 13-HPODE (Su et al., 2000), the bis-allylic 11-HPODE represents with $~ 75 \%$ of all products clearly the major product of the CspLOX2 peroxidation reaction.

Table 11. Comparison of reaction characteristics and kinetics of $13 R-M n L O X$ and CspLOX2.

CspLOX2

13R-MnLOX

\begin{tabular}{ccc}
\hline Abstracted hydrogen & Pro-S (Andreou et al., 2010) & Pro-S (Hamberg et al., 1998) \\
\hline $\begin{array}{c}\text { Side of oxygen insertion } \\
\text { relative to hydrogen } \\
\text { abstraction }\end{array}$ & $\begin{array}{c}\text { Antarafacial (Andreou et al., } \\
\text { 2010) }\end{array}$ & $\begin{array}{c}\text { Suprafacial (Hamberg et al., } \\
\text { 1998) }\end{array}$ \\
\hline
\end{tabular}

Ratio of bis-allylic to

conjugated products from linoleic acid during

75:25 29:71 (Su et al., 2000) peroxidation phase

\begin{tabular}{|c|c|c|}
\hline $\boldsymbol{k}_{\text {cat }}$ of peroxidation rate & 28.5 & 26 (Su et al., 2000) \\
\hline $\begin{array}{c}\boldsymbol{k}_{\text {cat }} \text { of 11-HPODE formation } \\
\text { from linoleic acid }\end{array}$ & 23 & 7 (Su et al., 2000) \\
\hline $\begin{array}{c}k_{\text {cat }} \text { of 11-HPODE } \\
\text { isomerization to conjugated } \\
\text { products }\end{array}$ & 0.6 & 9 (Su et al., 2000) \\
\hline $\begin{array}{c}\text { 11-HPODE isomerization has } \\
\text { a kinetic lag phase }\end{array}$ & no & $\begin{array}{c}\text { yes (Oliw et al., 2004; Su et } \\
\text { al., 2000) }\end{array}$ \\
\hline $\begin{array}{l}\text { Number of reaction phases } \\
\text { with linoleic acid visible at } \\
234 \mathrm{~nm}\end{array}$ & 2 & $\begin{array}{c}\text { only } 1 \text { (Hamberg et al., 1998; } \\
\text { Su et al., 2000) }\end{array}$ \\
\hline $\begin{array}{l}\text { Parallel formation of bis- } \\
\text { allylic and conjugated } \\
\text { HPODEs }\end{array}$ & yes & $\begin{array}{c}\text { yes (Hamberg et al., 1998; Su } \\
\text { et al., 2000) }\end{array}$ \\
\hline
\end{tabular}

Active site metal

Iron: Fe(II) and Fe(III)

Manganese: $\mathrm{Mn}(\mathrm{II})$ and $\mathrm{Mn}(\mathrm{III})$ (Su et al. , 2000)

As the formation of bis-allylic products by other iron-containing LOXs may have been overseen due to suboptimal extraction conditions and the instability of the product (Figure 23 and Figure 24), four different iron-containing LOXs from plants (sLOX1, CsLbLOX), bacteria (CspLOX1) and fungi (FoxLOX) were tested for their ability to form 11-HPODE from linoleic acid. But even under optimized conditions, none of the tested enzymes produced the bis-allylic product, suggesting that 11-HPODE formation is indeed an unusual phenomenon that is limited to few iron- 
containing LOXs. Nevertheless, two of the four tested enzymes, CspLOX1 and FoxLOX, were able to isomerize 11-HPODE to conjugated HPODEs (Figure 31). The obtained product distributions are in line with the products obtained with linoleic acid. The 9-LOX CspLOX1 (Newie et al., 2015) produces mainly 9-HPODE and the 13-LOX FoxLOX (Brodhun et al., 2013) produces mainly 13HPODE from 11-HPODE. These results suggest that the isomerization reaction has high similarity to the peroxidation reaction and supports the hypothesis that the isomerization occurs via deoxygenation and subsequent reoxygenation (Hamberg et al., 1998) (Figure 54). Linoleic acid and 11-HPODE probably bind to the active site in a very similar way and thus oxygen is directed to the same position after deoxygenation of 11-HPODE.

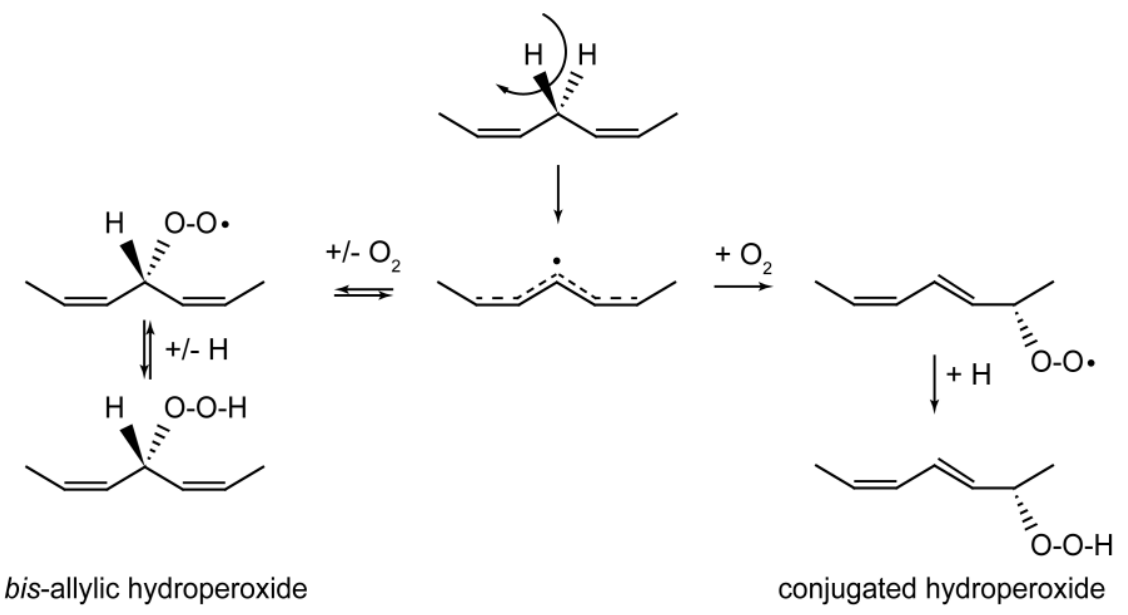

Figure 54. Mechanism proposed for the peroxidation and isomerization of PUFAs by CspLOX2 and MnLOXs. After abstraction of the hydrogen atom from the middle position, oxygen can either be inserted at the middle position to form the bis-allylic hydroperoxide or at the end position to form a conjugated hydroperoxide. Isomerization of the bis-allylic hydroperoxide probably occurs via deoxygenation and formation of the carbon centered radical (Hamberg et al., 1998).

Since the most obvious difference between CspLOX2 and MnLOXs is the metal species in the active site, the role of the cofactor was also analyzed. Initial EPR experiments with CspLOX2 suggested that in addition to iron, also manganese might be present in the active site. It thus seemed possible that CspLOX2 contains a portion of manganese that might be responsible for the 11-HPODE formation. The oxygenation specificity at the bis-allylic carbon could thus be related to the manganese cofactor. Therefore, CspLOX2 variants containing either manganese or iron as catalytic metal were generated by expression in minimal medium. Kinetic analyses showed that only Fe-CspLOX2 catalyzed the dioxygen insertion into linoleic acid (Table 8). The negligible activity of Mn-CspLOX2 is most likely caused by a residual amount of iron in the active site ( $2 \mathrm{~mol} \%$, Table 8 ). It is apparent, that only iron but not manganese can function as catalytic metal in CspLOX2. These results confirm that 11-HPODE can indeed be formed by an ironcontaining enzyme and that the manganese cofactor is not the important key for 11-HPODE formation.

So far, it was assumed that iron and manganese cofactors function very similarly in LOXs. Both metals are coordinated by three histidines, an asparagine and a carboxy group of the C-terminal 
valine or isoleucine (Supplemental Figure 4). The results obtained in this study also show that the expression conditions have an influence on the metal in the active site. In the crystal structure of Mn-CspLOX2, a full occupancy of the metal cofactor was found in the active site (Figure 33). This suggests that manganese is not only unspecifically bound to the surface but indeed integrated into the iron-binding site with the same octahedral coordination as the iron cofactor.

It is also known from other enzymes that $\mathrm{Mn}$ (II) and Fe(II) prefer similar coordination spheres which makes it difficult for the enzyme to distinguish between both metals based on the structure alone. But since the midpoint potentials of $\mathrm{Mn}(\mathrm{II}) / \mathrm{Mn}$ (III) and $\mathrm{Fe}(\mathrm{II}) / \mathrm{Fe}(\mathrm{III})$ of the hexaaquo ions are quite different with 1.5 and $0.8 \mathrm{~V}$, respectively, the redox chemistry of both metals is not interchangeable (Cotruvo Jr and Stubbe, 2012; Dean, 1985). In SODs, similar but not identical active sites are necessary to adjust the redox potentials to allow both, the reduction and oxidation of $\mathrm{O}_{2}{ }^{--}$(Borgstahl et al., 2000; Cotruvo Jr and Stubbe, 2012; Lah et al., 1995). This might also be the case for MnLOXs and FeLOXs. Interestingly, the oxidation of $\mathrm{Mn}$ (II) to $\mathrm{Mn}$ (III) was also observed in the CspLOX2 active site and even if Mn-CspLOX2 is unable to form the hydroperoxide product (Figure 32). However, while iron is converted from Fe(II) to $\mathrm{Fe}(\mathrm{III})$ in the presence of HPODE, it will finally react back to Fe(II) after one minute with linoleic acid (Figure 32). In contrast to this, $\mathrm{Mn}(\mathrm{II})$ is converted to $\mathrm{Mn}$ (III) with HPODE, but also with linoleic acid. It might therefore be possible, that due to the different redox properties of manganese and iron, manganese cannot complete the full reaction cycle.

Structural data of MnLOXs will be finally required to elucidate why Mn-CspLOX2 is inactive while fungal MnLOXs catalyze the same reaction with catalytically active manganese in a presumably identical coordination sphere. Furthermore, it will be interesting if MnLOXs are catalytically functional with iron in the active site. It should also be noted that our data suggest that the host organism and expression conditions have a crucial influence on the metal incorporation. As all MnLOXs were expressed in P. pastoris instead of E. coli as most other characterized LOXs (Cristea et al., 2005; Su and Oliw, 1998; Wennman and Oliw, 2013; Wennman et al., 2015), the question arises whether the catalytic metal found in heterologously expressed LOXs is identical with the one in the original host organism or whether only the expression system determines the type of metal that is incorporated. It has also been shown in other studies that overexpressed proteins are sometimes isolated with an incorrect metal center (mismetallation) (Maret, 2010). And it has also been suggested for other enzymes that the metal availability rather than a structural preference discriminates between iron and manganese (Cotruvo Jr and Stubbe, 2012). It was for instance shown by Waldron and coworkers that $E$. coli favors the incorporation of iron into the MnSOD of Bacillus anthracis (Tu et al., 2012). The expression of MnLOX in P. pastoris might therefore also be necessary to ensure the correct metallation of MnLOX. Other manganesecontaining enzymes like a peroxidase have also been successfully expressed in this $P$. pastoris (Gu et al., 2003). 


\subsection{The most important factors for 11-HPODE formation in CspLOX2 may include distortion of the pentadiene and steric hindrance at C9 and C13}

Kinetic and spectroscopic analyses suggested that the type of metal cofactor is not responsible for 11-HPODE formation and instead steric factors of the amino acid environment are probably crucial for this specificity. CspLOX2 appeared to be an ideal candidate to study the molecular basis of this reaction. A structure with a well-defined substrate binding channel is available and it is the LOX with the highest amount of bis-allylic product known so far.

Although the synthesis of 11-HPODE is an unusual phenomenon and only few LOXs are able to form this product, there are some interesting aspects about this reaction. On the one hand, the insertion of oxygen at the bis-allylic carbon atom is thermodynamically not favorable and this product is in general not formed by autoxidation (Chan and Levett, 1977; Porter, 1990). It is therefore interesting, how the enzyme can lower the activation barrier for the oxygen insertion at C11 of linoleic acid. On the other hand, the principles that direct dioxygen to the bis-allylic position may also be applicable to explain how the specificity for only one of the four conjugated products on one pentadiene system is achieved by classical LOXs.

A comparison of the effects resulting from many mutations along the CspLOX2 substrate binding channel led to the conclusion that especially those amino acids located in the core of the CspLOX2 active site play a decisive role in directing dioxygen to different positions on the linoleic acid substrate (Figure 34). Interestingly, the control of LOX specificity has mainly been considered to be determined by the bottom of the active site channel. In one model, the depth of the active site is regulated by two residues residing at the bottom of the pocket (Sloane et al., 1991). Depending on the size of these residues, different pentadiene systems can be exposed to the reactive iron (Figure 10). In addition to the depth of the substrate binding, the orientation of the substrate was also discussed to be determined by amino acid residues located at the bottom of the channel. Positively charged residues, especially a conserved arginine, have been associated with a reversed substrate orientation due to interactions with the negatively charged carboxyl head group (Hornung et al., 1999). The results of the present study suggest that more attention should be paid to the direct environment of the reacting pentadiene moiety in the active site. Exchanging residues in this area resulted in significant changes of the product composition. The specificity of individual CspLOX2 variants could be directed to all four possible conjugated products of linoleic acid: 9S-, 9R-, 13S- and 13R-HPODE. So far, a major function has only been attributed to the so-called Gly/Ala switch in the active site (Coffa and Brash, 2004). A glycine at this position is related to $R$-specific LOXs while a bulkier alanine at this position is in general related to $S$-specific lipoxygenation. This Gly/Ala switch is also part of the identified crucial residues in CspLOX2.

To elucidate which role the exchanged amino acids might play, four different hypotheses were considered (Figure 55) which are based on the concepts proposed for the general oxygenation 
specificity of LOX and COX enzymes (Schneider et al., 2007). Since insertion of oxygen at the bisallylic position of the substrate represents simply an "unusual" oxygenation specificity on the pentadiene system, the models might also be useful to identify a molecular basis of the 11HPODE formation.

The first model explains the altered specificity by torsion of the pentadienyl radical (Figure 55A). The idea is that a reaction at $\mathrm{C} 11$ becomes feasible upon slight torsions of the dihedral angle $\varphi$. In that case, the pentadiene system would not be in plane anymore and the delocalization of the radical would be limited. The radical would thus preferentially stay at the middle position of the pentadiene.

In the second model, the final reduction step of the peroxyl radical to the hydroperoxide is the important factor (Figure 55B). Since the formed peroxyl radical can undergo quick $\beta$ fragmentation at the bis-allylic position (Figure 12), oxygen insertion is in principle reversible. The peroxyl radical on $\mathrm{C} 11$ could, however, be trapped by an appropriate hydrogen atom donor in the direct amino acid environment to form the more stable hydroperoxide, thereby preventing $\beta$-fragmentation (Pratt et al., 2003).

In the third model, an oxygen channel plays a decisive role (Figure 55C). This channel would transport molecular oxygen from the surface of the protein directly to the active site. If the oxygen channel enters the substrate binding channel directly at C11 of linoleic acid, oxygen may be preferentially inserted here.

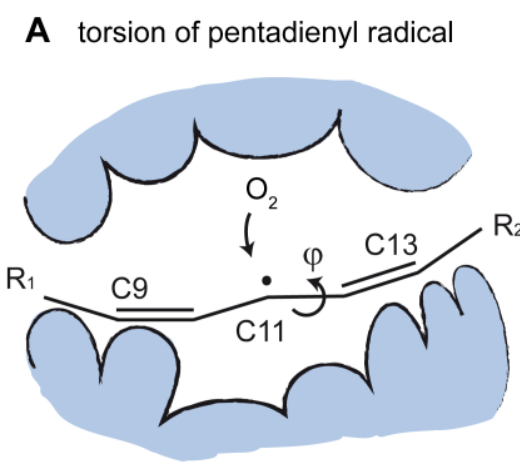

B selective peroxyl
radical trapping

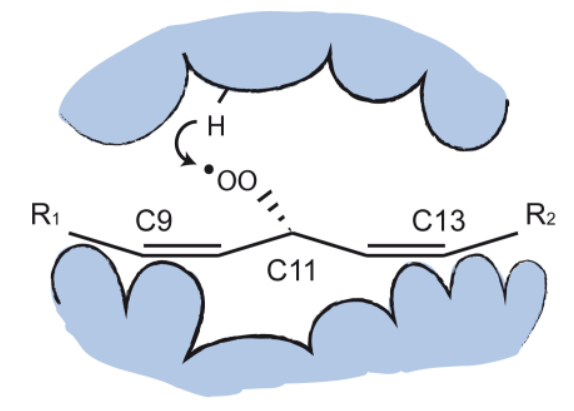

C oxygen channel

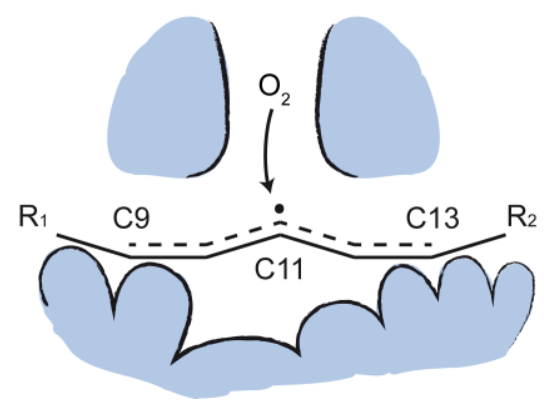

D steric shielding

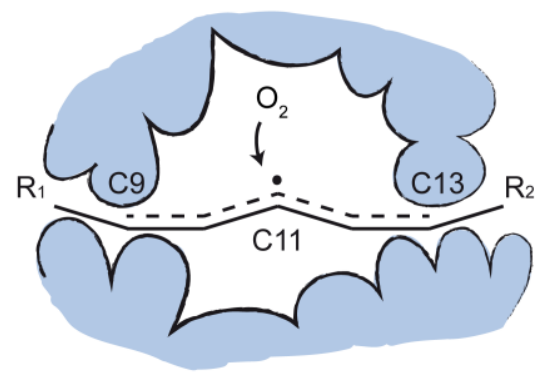

Figure 55. Four models to explain the oxygenation specificity on $\mathrm{C} 11$ of linoleic acid. The models are based on the ideas for the LOX and COX specificity as published by Schneider et al. (Schneider et al., 2007). 
In the last model, steric shielding might be important to direct dioxygen to C11 (Figure 55D). Amino acids located close to $\mathrm{C} 9$ and $\mathrm{C} 13$ on linoleic acid restrict the space at these positions during LOX catalysis. Consequently, no oxygen can reside here and no space is available to form the bulky hydroperoxide at these positions. Therefore, the reaction would specifically occur on C11 where more space is available.

\subsubsection{Radical localization}

The residues involved in the most pivotal changes of the product composition form a tight clamp-like structure around the CspLOX2 active site. Of this active site clamp, Leu304 plays a special role. Exchanging this residue for a smaller valine resulted in the lowest amount of 11HPODE product among the analyzed CspLOX2 variants. This leucine residue is located opposite of the iron and thus restricts the channel diameter directly at the catalytic iron (Figure 36). Leu304 is highly conserved among all LOXs, demonstrating its crucial role for LOX catalysis. Nevertheless, the diameter of the active site channel between iron and this leucine residue varies in different LOXs, suggesting a slightly different location of the arched helix that contributes this residue (Figure 37). Leu304 might apply a certain strain on the linoleic acid substrate in the tight and kinked channel of CspLOX2, thereby inducing slight torsions of the dihedral angle in the pentadiene system. This would be required to achieve a radical localization as illustrated in Figure 55A. Since no structure of a CspLOX2 substrate complex could be obtained, the effect of torsions in the dihedral angle was evaluated by means of theoretical chemistry. Although slight shifts of the spin densities were obtained depending on the dihedral angle, these shifts were not sufficient to explain the experimentally observed product distributions (Figure 38 and Figure 50C).

But even more interesting than the localization of the radical is the question if the activation barrier at C11 can be lowered by torsion of the pentadiene system. In comparison to 9-HPODE and 13-HPODE, 11-HPODE is in general not observed as autoxidation product of linoleic acid (Chan and Levett, 1977; Frankel et al., 1977; Porter, 1990). This implies that the activation barrier of this product is much higher at this position compared to $C 9$ and $C 13$. Although the spin density is slightly higher at C11 which generally indicates that oxygen is preferentially added here (Figure 50 ), the Gibbs free energy of oxygen addition favors the end position of the pentadiene, as $\Delta G^{0}$ is more negative for the end positions $\left(-1.9 \mathrm{kcal} \mathrm{mol}^{-1}\right)$ compared to the central position $\left(+3.7 \mathrm{kcal} \mathrm{mol}^{-1}\right.$ ) (Wright et al., 2009). This phenomenon was explained by the energy gain due to the formation of conjugated double bonds in case of oxygen addition to the end position of the pentadienyl radical, while the conjugated system of the delocalized radical is lost by addition of oxygen to the middle position (Wright et al., 2009).

The theoretical calculations of the present study further showed that the Gibbs free energy for the oxygen insertion can be substantially lowered at the middle position of the pentadienyl radical by slight torsions of only $20^{\circ}$ (Figure 50D). In this case, the overlap of the $\pi$-orbitals in the 
conjugated system might be lowered and thus the effect of conjugation is decreased compared to a planar system. These results suggest that torsions of only a few degrees might also be necessary for the CspLOX2 reaction on C11 of linoleic acid. It is most likely a prerequisite to make the oxygen insertion at $\mathrm{C} 11$ competitive to $\mathrm{C} 9$ and $\mathrm{C} 13$. It would be possible that the tight and kinked channel together with Leu304 positioned in the kink apply a force onto the pentadiene moiety, thereby evoking this torsion.

Notably, this position was also exchanged in 13R-MnLOX. Here, Leu336 (Leu304 in CspLOX2) was exchanged for valine, alanine, glycine and phenylalanine (Wennman et al., 2012). However, it is difficult to compare the results with CspLOX2, since only the relative amount of 9-HPODE and 13-HPODE produced by these variants was determined, but not the amount of 11-HPODE. Furthermore, the Leu336Val variant retained about $48 \%$ of the wt activity, but the Leu336Ala and Leu336Gly variant were almost inactive with only $<0.2 \%$ of the wt activity. The most pronounced effect on the specificity of the enzyme was nevertheless identified for the Leu336Ala variant which formed 13-HPODE and 9-HPODE in a ratio of $39 \%$ and $61 \%$, respectively, whereas the wt produces almost exclusively 13-HPODE (Wennman et al., 2012). As no information about 11-HPODE produced by these variants has been provided, the function of Leu336 in 13R-MnLOX for the formation of the bis-allylic product remains unknown.

\subsubsection{Selective peroxyl radical trapping}

Studies on fatty acid autoxidation have shown that conjugated hydroperoxides of mainly trans, trans diene geometry are produced in oxidation reactions without any antioxidants (Porter and Wujek, 1984). If millimolar concentrations of phenolic antioxidants are present, for instance $\alpha$ tocopherol, cis, trans hydroperoxides are the major products. In autoxidation reactions of methyl linoleate in the presence of $\sim 0.1 \mathrm{M} \alpha$-tocopherol, the bis-allylic 11-hydroperoxide was also identified as product (Brash, 2000; Tallman et al., 2001). It was suggested that one principle to understand the product distribution of linoleic acid oxidations is the reversibility of the dioxygen addition to pentadienyl radicals. In these autoxidation reactions, the loss of oxygen from peroxyl radicals ( $\beta$-fragmentation) competes with hydrogen atom trapping of these peroxyl radical by $\alpha$-tocopherol (Pratt et al., 2003). It should be noted, that the $\beta$-fragmentation occurs at a much higher rate at the middle position $\left(2.4 \times 10^{6} \mathrm{~s}^{-1}\right)$ (Tallman et al., 2001) compared to the end positions of the pentadiene $27 \mathrm{~s}^{-1}$ (Porter and Wujek, 1984). Therefore, peroxyl radical trapping might be especially important for the bis-allylic product.

These considerations were transferred to the CspLOX2 reaction. Since the presence of high concentrations of antioxidants leads to 11-HPODE formation in solution, it is conceivable that suitable hydrogen atom donors in the environment of the C11 peroxyl intermediate could trap the product at this position and prevent $\beta$-fragmentation. Amino acid side chains that could potentially function as antioxidants by donating hydrogen atoms include tyrosine, tryptophan or cysteine (Giles et al., 2003; Li et al., 1991; van Overveld et al., 2000). However, none of these 
amino acids was found in the suitable position within the CspLOX active site. Only the side chain of Tyr310 was found in a $10 \AA$ radius around the pentadiene system. As no tyrosyl radical was detected by EPR spectroscopy and the exchange of this residue for isoleucine and phenylalanine had no effect on the 11-HPODE formation, a potential catalytic function of Tyr310 was disfavored. Furthermore, such tyrosine radicals would be very reactive themselves and probably lead to a damage of the enzyme. The question how the peroxyl radical is scavenged in the last step of the LOX reaction cycle is still a matter of discussion. Formally, it would be consistent with the reaction cycle if the hydrogen atom that was initially abstracted by the ferric iron is transferred back to the peroxyl radical in order to complete the reaction cycle (Tomchick et al., 2001). This, however, implies that the fatty acid has to be rearranged after oxygen insertion. As oxygen is in general inserted at the $n+2$ or $n-2$ position relative to the hydrogen abstraction in an antarafacial way, the fatty acid peroxidyl radical needs to turn $180^{\circ}$ and align the peroxyl group to the $\mathrm{H}_{2} \mathrm{O}$ coordinated by the ferric iron (Figure 56). Although this appears inconvenient, no other path has been identified so far that could explain how the peroxyl radical is finally trapped.

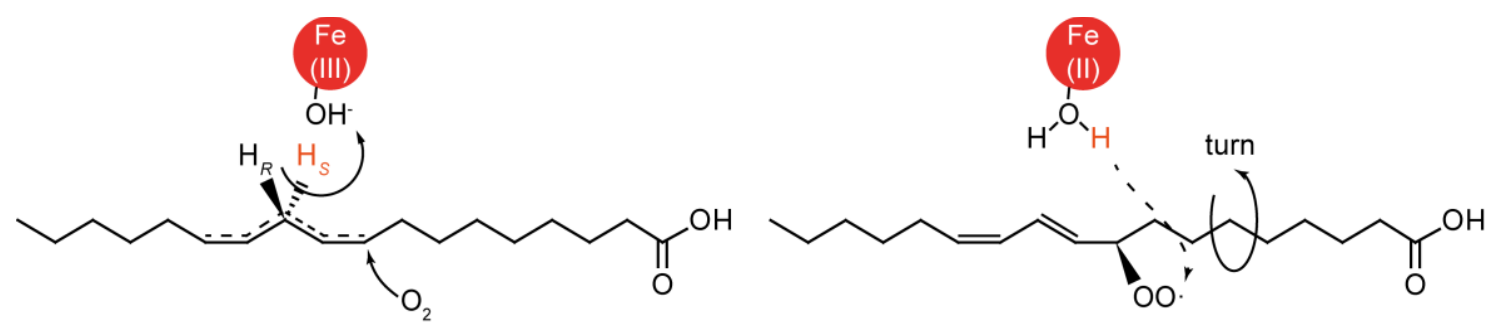

Figure 56. Reduction of the peroxyl radical is assumed to be mediated by the iron coordinated water (Tomchick et al., 2001). For this process, the substrate would need to turn and align with the iron cofactor to bring the peroxyl radical in proximity to the hydrogen atom.

\subsubsection{Oxygen channel}

Another possibility to direct molecular oxygen to the middle position of the pentadienyl radical would be an oxygen channel that guides the gas molecules from the surface of the enzyme to the active site, thereby increasing the local concentration of molecular oxygen. The existence of such an oxygen channel has been postulated for different LOX enzymes based on crystal structures and mutational studies (Knapp et al., 2001; Kühn et al., 2005; Minor et al., 1996). The recently published structure of the $8 R$-LOX in complex with arachidonate also revealed a channel that connects the surface of the protein with the substrate binding channel (Figure 46). Assuming that this channel indeed functions as oxygen channel, molecular oxygen would directly be transported to the middle of the reacting pentadiene system of the substrate opposite of the iron. The location of such a channel is therefore in line with the antarafacial relationship of hydrogen abstraction and oxygen insertion. This putative oxygen channel which was also found in the structure of porcine 12-LOX and the human 15-LOX-2 (Neau et al., 2014) was also identified in the structure of CspLOX2 (Figure 46). As xenon derivatization experiments as well as mutational studies did not lead to a direct evidence for the existence of this oxygen channel 
(Figure 47), the function of this tunnel remains hypothetical. It should, however, be noted that this channel would have the capacity to increase oxygen concentrations specifically in the environment of the pro- $R$ side of $\mathrm{C} 11$ and could thus contribute to the $11 R$-HPODE formation by CspLOX2.

\subsubsection{Steric shielding}

The tight substrate binding channel of CspLOX2 that is surrounded by mainly leucine residues led to the assumption that steric shielding is an important factor to explain the CspLOX2 specificity, which was supported by mutagenesis studies. Decreasing the size of amino acid residues around C9 of the substrate increased the formation of 9-HPODE, while 13-HPODE formation was increased by reducing the size of amino acids around C13. More space at one of the potential reactive sites $(9 S, 9 R, 13 S$ and $13 R)$ shifted the product ratio towards this particular product. At $C 9$, the correlation between the $S$ - and $R$-enantiomer and the site of mutation seems clear, with lle296 being located on the $S$-side and Ala300 being located on the $R$-side. Around C13, a similar correlation could be observed, although it is less obvious. Leu304 and Leu502 are rather facing the $R$-side and Leu506 the $S$-side of C13 (Figure 38). As no crystal structure of the CspLOX2 substrate complex could be obtained, these conclusions are only based on the arachidonic acid structure crystallized in complex with $8 R$-LOX.

In order to get a better understanding of the role of steric hindrance for 11-HPODE formation, a LOX with only conjugated products was converted into a LOX with bis-allylic products by mimicking the tight active site channel of CspLOX2. CspLOX1 was an ideal candidate for this gain of function approach as a crystal structure of the enzyme is available, the enzyme is the isozyme from Cyanothece sp. PCC8801 and it produces only the conjugated 9R-hydroperoxide (Newie et al., 2015). The exchange of one to two active site amino acids probably close to C9 and C13 of linoleic acid for slightly bulkier amino acids was sufficient to induce 11-HPODE formation (Figure 43). These results support the hypothesis that limited space around C9 and C13 may limit oxygen insertion to $\mathrm{C} 11$ as only reactive site.

The role of steric hindrance in 11-HPODE formation was further supported by simulations, in which only accessible sites on the substrate were regarded as reactive (Figure 51). Whenever another atom, for example from amino acid side chains was getting to close to $9 R, 11 R$ or $13 S$, the position was defined as unreactive. The distribution of reactive sites obtained from many simulations and snapshots exhibited a nice correlation with the experimentally observed product distributions of the CspLOX2 variants with amino acid substitutions on the antarafacial side of the substrate (Leu258Val, Ala300Gly, Leu304Val, Leu502Val) (Figure 51B). However, this model cannot yet explain the change of stereospecificity observed for the lle296Ala and Leu506Val variants. On the one hand it might be possible that due to the increased space oxygen would migrate to the backside of the substrate where oxygen is inserted in a suprafacial way (Figure 57A). On the other hand, one half of the pentadiene system could adopt another 
conformation by rotation around the axis of the fatty acid since more space is available (Figure $57 B)$.
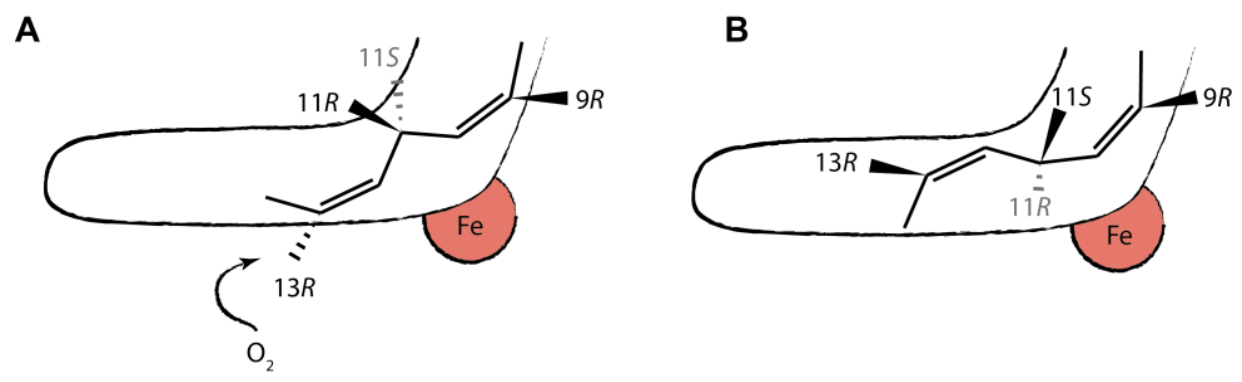

Figure 57. Two models may explain oxygen access to $13 R$ of linoleic acid. (A) The first option is that oxygen does not attack from the opposite site of the iron, but rather migrates to the backside of the pentadiene to insert oxygen in a suprafacial way. (B) Another option is that the pentadiene is twisted in a way that the side for $13 R-H P O D E$ formation is exposed to the front. The same models may explain how oxygen is inserted to form 9S-HPODE.

Taken together, these results suggest that the reacting pentadiene system of the substrate needs to be slightly distorted to make an oxygen insertion at C11 of linoleic acid favorable. This might be induced by Leu304 being located in the kink of the channel and is probably the prerequisite to explain the reaction at the bis-allylic position. Additionally, a putative oxygen channel was identified that might deliver dioxygen precisely to the site of $11 R$-HPODE formation. Direct evidence for the function of this channel in oxygen transport is, however, missing. Steric shielding was identified as most promising factor to inhibit oxygen insertion at C9 and C13 of linoleic acid, while leaving the site for 11R-HPODE formation accessible for dioxygen.

\subsection{The concepts may also apply to MnLOXs and classical LOXs with only conjugated products}

It is now interesting to evaluate whether the mechanisms that regulate the oxygenation specificity of CspLOX2 are also applicable to the formation of the bis-allylic product by MnLOXs. Similarities in the reaction mechanism were found like the two individual reactions of fatty acid peroxidation and 11-HPODE isomerization (Table 11) which may point to a similar molecular strategy. As no structural data of a MnLOX is available so far, the effect of steric shielding is rather vague. Nevertheless, some information was obtained by mutational studies within the 13R-MnLOX active site. It was suggested that Phe337 (Ile 305 in CspLOX2) sterically shields the $13 R$-side of linoleic which may explain the formation of $13 S$-HPODE in a suprafacial reaction (Wennman et al., 2012). Here, molecular oxygen probably also needs to move to the opposite side of the substrate to insert dioxygen at the suprafacial side where the iron is located. This would be in favor of model A shown in Figure 57. Steric shielding might thus also be one of the most important factors to explain the 11-HPODE formation by MnLOXs. Since MnLOXs abstract the pro-S hydrogen on C11 and directly insert oxygen at the same position to form 11S-HPODE, peroxyl radical trapping by the close $\mathrm{Fe}(\mathrm{II})-\mathrm{H}_{2} \mathrm{O}$ may play an additional role. Furthermore, the pentadiene of the linoleic acid substrate modeled into the predicted 3D model of $13 R$-MnLOX 
also exhibited a slightly twisted conformation (Wennman et al., 2012). Although such predictions are only rough approximations, this may suggest that torsions of the pentadiene are necessary to allow oxygen insertion at C11 of linoleic acid which is in line with the results of the present study.

Moreover, the molecular principles of the CspLOX2 reaction may even apply to classical LOXs with only conjugated products to explain the high stereo- and regiospecificity of the oxygen insertion within one pentadiene system. If enough space is available in the active site, for example by a larger distance of the conserved leucine (Leu304 in CspLOX2) to the catalytic iron (Figure 36), a planar pentadienyl radical will be favored due to the energy gain from a conjugation effect over all five carbon atoms. As the energy barrier is higher at the middle position (Figure 50D) compared to the end positions of the pentadienyl radical in a planar system, oxygen insertion will be strongly favored at C9 or C13. This might be the reason why 11HPODE is generally not observed as LOX product. Additionally, the putative oxygen channel identified in CspLOX2 seems to be conserved in many animal and coral LOX structures and might thus explain the antarafacial relationship of hydrogen abstraction and oxygen insertion (Neau et al., 2014).

The effect of steric shielding probably also plays a major role in classical LOXs. The Gly/Ala switch was already found as one of the major determinants in proximity to the reacting pentadiene. Controlling the oxygen insertion to $9 R$ and $13 S$ of linoleic acid was also associated with steric hindrance at C9 (Coffa and Brash, 2004; Neau et al., 2014). But also other studies have discussed steric shielding as mechanism to explain the specificity of the LOX reaction. Leu546 and Leu754 of soybean LOX1, which correspond to Leu304 and Leu506 in CspLOX2 respectively, have been proposed to be involved in steric shielding as well (Knapp et al., 2001; Knapp and Klinman, 2003). Leu597 (Leu506 in CspLOX2) has also been exchanged for smaller amino acids in the rabbit 15LOX and resulted in higher amounts of the $15 R$-hydroperoxide which corresponds to the $13 R$ hydroperoxide of linoleic acid (Suardíaz et al., 2013; Suardíaz et al., 2014). These observations parallel the effect of the Leu506Val substitution in CspLOX2 and were also explained with steric shielding (Saura et al., 2014; Suardíaz et al., 2013; Suardíaz et al., 2014). Therefore, it is feasible that steric shielding is also one of the major mechanisms to direct the oxygen insertion within one reacting pentadiene system in other LOXs, including plant and mammalian enzymes.

Models that explain the selection of a certain pentadiene moiety by the depth of the substrate binding channel and the side of oxygen insertion with the orientation of the substrate (tail-first or reversed) have been well established. In these models, not only the oxygen insertion, but also the position of hydrogen abstraction is affected (Figure 10). The results of this study now complement these models by explaining how oxygen can be delivered to different positions on the pentadienyl radical without changing the targeted pentadiene system nor reversing the substrate orientation and thereby leaving the site of hydrogen abstraction unaffected. The here presented data suggest that in addition to the known Gly/Ala switch, other residues around the 
active site probably shield particular reactive sites from molecular oxygen, thereby achieving selective oxygen insertion on another position. It would now be highly interesting to elucidate if the steric shielding by the active site clamp plays indeed a major role in all LOXs.

\subsection{Outlook}

In this study, no structure of a CspLOX2 substrate complex could be obtained. Such a crystal structure would, however, be essential to support the hypothesis that the pentadiene system needs to be slightly twisted to allow oxygen insertion at the bis-allylic position. Extensive trials performed within this work showed that using CspLOX2 for this approach is very challenging. A possible reason why the substrate did not bind in the active site during crystallization or soaking might be the difference between the optimal $\mathrm{pH}$ for the CspLOX2 reaction ( $\mathrm{pH}$ 9-9.5) and the $\mathrm{pH}$ of the crystallization conditions ( $\mathrm{pH}$ 6-6.5). In $8 \mathrm{R}$-LOX, both $\mathrm{pH}$ values were closer together (optimal pH 7.5 and pH 8 for crystallization). It might therefore be worth to try crystallization of an enzyme substrate complex with another LOX that forms bis-allylic products. Another option is to increase the 11-HPODE formation by $8 R$-LOX in a similar approach as performed in this study with CspLOX1 (Chapter 3.6). 8R-LOX seems to be more suitable for obtaining crystals of enzyme substrate complexes. The structure of the variant in complex with substrate could subsequently be compared with the already existing structure of $8 R$-LOX with arachidonate.

Furthermore, for evaluating the role of steric shielding in LOXs in general, the effect of the active site clamp mutations could also be investigated in other LOX enzymes. In combination with biochemical data of the product specificity, the effect of steric shielding could be analyzed using the computational approach developed by Martin Werner and Prof. Dr. Ricardo Mata. So far, the inversion of the stereochemistry cannot yet be explained with the existing models. Further theoretical and experimental studies might be necessary to understand how the stereochemistry of 9R-HPODE and 13S-HPODE can be inverted by single point mutations.

To evaluate if torsions of the pentadiene might indeed lead to a localization of the radical on C11 of linoleic acid, time-resolved EPR spectra could be recorded (Schneider et al., 2007; Schreiner and Berndt, 1974). However, for the reaction of COX-2, which also forms a pentadienyl radical, a localized radical could not be found (Tsai et al., 2002). The problem with LOXs is that they are usually too fast with a $k_{\text {cat }}$ of up to $300 \mathrm{~s}^{-1}$ for soybean LOX1. The CspLOX2 reaction, however, proceeds at a rate that is about 10 times slower. It might thus be worth to use CspLOX2 for these measurements instead.

Also the role of the cofactor left some open questions. It would be interesting to elucidate, whether MnLOXs can also be substituted by iron and if they would still be active. At the same time, a crystal structure of manganese LOX could reveal the detailed coordination geometry of this cofactor and special features of the active site which may explain why these enzymes are active with manganese while CspLOX2 is not. 


\section{Summary}

Lipoxygenases (LOXs) are non-heme iron- or manganese-containing dioxygenases that initiate the biosynthesis of vital lipid mediators in mammals and plants. They catalyze the regio- and stereospecific peroxidation of polyunsaturated fatty acids containing at least one cis,cis-1,4pentadiene system. In previous studies, two iron-containing LOX isozymes, CspLOX1 and CspLOX2, have been identified in Cyanothece sp. and their crystal structures have been solved. In this study, both enzymes were characterized in detail to elucidate the relationship between protein structure and function. Most interesting were thereby the $\mathrm{N}$-terminal $\alpha$-helical extension of CspLOX1 which is not present in other LOXs and the unusual oxygenation specificity of CspLOX2.

Besides the catalytic LOX domain and an N-terminal $\beta$-barrel domain found in eukaryotic LOXs, CspLOX1 harbors an additional $\alpha$-helical extension at the $\mathrm{N}$-terminus with unknown function. The $\beta$-barrel domain has generally been proposed to mediate a membrane binding function. However, the here described liposome binding studies with truncated CspLOX1 versions revealed that only the $\alpha$-helical extension but not the $\beta$-barrel is crucial for binding of CspLOX1 to the vesicles. This domain might thus present a new membrane targeting domain that could be involved in direct substrate acquisition from membranes.

Furthermore, CspLOX2 represented an ideal model to study the dioxygen insertion at the middle position of the pentadiene that leads to the formation of bis-allylic hydroperoxides: A crystal structure of CspLOX2 is available and chromatographic and kinetic analyses of this study showed that CspLOX2 is the enzyme with the highest relative amount of the bis-allylic product observed so far. This unusual product had previously only been reported for manganese-containing LOXs, while iron-containing LOXs generally insert oxygen only at the ends of the pentadiene system. What role the type of metal cofactor plays for the LOX reaction was not understood. Therefore, a manganese-substituted version of CspLOX2 (Mn-CspLOX2) was generated in this study by expression of the enzyme in minimal medium. Despite a virtually identical coordination geometry of manganese in the metal binding site, Mn-CspLOX2 was inactive, indicating that iron and manganese cofactors are not interchangeable in LOXs.

Since manganese as metal cofactor is not required for the formation of bis-allylic products, structural factors were considered to explain this oxygenation specificity. CspLOX2 exhibits a substrate binding channel with a smaller diameter than other LOXs. Increasing the space in the active site by exchanging Leu304 for valine decreased the relative amount of the bis-allylic 11hydroperoxy octadecadienoic acid (HPODE) formation from linoleic acid substantially. Substitution of other amino acids in the core of the active site by smaller residues shifted the major products of linoleic acid to either $9 R-$, 9S-, 13R- or 13S-HPODE, depending on the amino acid position. Furthermore, in a gain of function approach, the active site of CspLOX1 was constricted by two point mutations to mimic the CspLOX2 active site. This in turn induced 11HPODE formation in CspLOX1. These results suggest that steric shielding plays an important role in the formation of the bis-allylic product which was also supported by a computational approach. Additionally, the confined active site may induce a torsion of the pentadiene system, thereby increasing the reactivity at C11. Steric shielding is probably also one of the most important factors to explain the oxygenation specificity of other LOXs. It is therefore likely that amino acids in the core of the active site play a more important role for controlling the oxygenation specificity than previously assumed. 


\section{References}

Aaij, C. and Borst, P. (1972). The gel electrophoresis of DNA. Biochim Biophys Acta, 269, 192200.

Adams, P. D., Afonine, P. V., Bunkoczi, G., Chen, V. B., Davis, I. W., Echols, N. and other authors (2010). PHENIX: a comprehensive Python-based system for macromolecular structure solution. Acta Cryst, D66, 213-221.

Aharony, D. and Stein, R. L. (1986). Kinetic mechanism of guinea pig neutrophil 5lipoxygenase. J Biol Chem, 261, 11512-11519.

An, J.-U., Kim, B.-J., Hong, S.-H. and Oh, D.-K. (2015). Characterization of an omega-6 linoleate lipoxygenase from Burkholderia thailandensis and its application in the production of 13-hydroxyoctadecadienoic acid. Appl Microbiol Biotechnol, 1-11.

Andreou, A.-Z., Vanko, M., Bezakova, L. and Feussner, I. (2008). Properties of a mini $9 R$ lipoxygenase from Nostoc sp. PCC 7120 and its mutant forms. Phytochemistry, 69, 1832-1837.

Andreou, A., Brodhun, F. and Feussner, I. (2009). Biosynthesis of oxylipins in non-mammals. Prog Lipid Res, 48, 148-170.

Andreou, A., Göbel, C., Hamberg, M. and Feussner, I. (2010). A bisallylic mini-lipoxygenase from cyanobacterium Cyanothece sp. that has an iron as cofactor. J Biol Chem, 285, 14178-14186.

Anjem, A., Varghese, S. and Imlay, J. A. (2009). Manganese import is a key element of the OxyR response to hydrogen peroxide in Escherichia coli. Mol Microbiol, 72, 844-858.

Ausubel, F. M., Brent, R. E., Kingston, D. D., Seidmann, J. R., Smith, J. A. and Struhl, K. (1993). Current Protocolls in Molecular Biology. New York: Green Publishing Associates and John Wiley and Sons Inc.

Baker, N. A., Sept, D., Joseph, S., Holst, M. J. and McCammon, J. A. (2001). Electrostatics of nanosystems: application to microtubules and the ribosome. Proc Natl Acad Sci USA, 98, 10037-10041.

Banthiya, S., Pekárová, M., Kühn, H. and Heydeck, D. (2015). Secreted lipoxygenase from Pseudomonas aeruginosa exhibits biomembrane oxygenase activity and induces hemolysis in human red blood cells. Arch Biochem Biophys, 584, 116-124.

Berg, J., Tymoczko, J. and Stryer, L. (2004). Biochemistry, 5th ed edn. New York: WH Freeman.

Bertani, G. (1951). Studies on lysogenesis. I. The mode of phage liberation by lysogenic Escherichia coli. J Bacteriol, 62, 293-300.

Bigay, J. and Antonny, B. (2005). Real-time assays for the assembly-disassembly cycle of COP coats on liposomes of defined size. Methods Enzymol, 404, 95-107.

Bimboim, H. and Doly, J. (1979). A rapid alkaline extraction procedure for screening recombinant plasmid DNA. Nucleic Acids Res, 7, 1513-1523.

Borgstahl, G. E., Pokross, M., Chehab, R., Sekher, A. and Snell, E. H. (2000). Cryo-trapping the six-coordinate, distorted-octahedral active site of manganese superoxide dismutase. $J$ Mol Biol, 296, 951-959.

Boutaud, O. and Brash, A. R. (1999). Purification and catalytic activities of the two domains of the allene oxide synthase-lipoxygenase fusion protein of the coral Plexaura homomalla. J Biol Chem, 274, 33764-33770.

Boyer, H. W. (1971). DNA restriction and modification mechanisms in bacteria. Annual Reviews in Microbiology, 25, 153-176.

Boyington, J. C., Gaffney, B. J. and Amzel, L. M. (1993). Structure of soybean lipoxygenase-I. Biochem Soc Trans, 21, 744-748.

Bradford, M. M. (1976). A rapid and sensitive method for the quantitation of microgram quantities of proteins utilizing the principle of protein-dye binding. Anal Biochem, 72, 248-254.

Brash, A. R. (1999). Lipoxygenases: Occurrence, functions, catalysis, and acquisition of substrate. J Biol Chem, 274, 23679-23682. 
Brash, A. R. (2000). Autoxidation of methyl linoleate: identification of the bis-allylic 11hydroperoxide. Lipids, 35, 947-952.

Brodhun, F., Göbel, C., Hornung, E. and Feussner, I. (2009). Identification of PpoA from Aspergillus nidulans as a fusion protein of a fatty acid heme dioxygenase/peroxidase and a cytochrome P450. J Biol Chem, 284, 11792-11805.

Brodhun, F., Cristobal-Sarramian, A., Zabel, S., Newie, J., Hamberg, M. and Feussner, I. (2013). An iron 13S-lipoxygenase with an $\alpha$-linolenic acid specific hydroperoxidase activity from Fusarium oxysporum. PLoS ONE, 8, e64919.

Browner, M. F., Gillmor, S. A. and Fletterick, R. (1998). Burying a charge. Nat Struct Biol, 5, 179.

Brünger, A. T. (1993). Assessment of phase accuracy by cross validation: the free $R$ value. Methods and applications. Acta Cryst, D49, 24-36.

Butovich, I. A., Lukyanova, S. M. and Reddy, C. C. (1998). Oxidation of linoleyl alcohol by potato tuber lipoxygenase: Possible mechanism and the role of carboxylic group in substrate binding. Biochem Biophys Res Commun, 249, 344-349.

Chan, H. W.-S. and Levett, G. (1977). Autoxidation of methyl linoleate: analysis of methyl hydroxylinoleate isomers by high performance liquid chromatography. Lipids, 12, 837840.

Chen, X. S. and Funk, C. D. (2001). The N-terminal " $\beta$-Barrel" domain of 5 -lipoxygenase is essential for nuclear membrane translocation. J Biol Chem, 276, 811-818.

Coffa, G. and Brash, A. R. (2004). A single active site residue directs oxygenation stereospecificity in lipoxygenases: Stereocontrol is linked to the position of oxygenation. Proc Natl Acad Sci USA, 101, 15579-15584.

Coffa, G., Imber, A. N., Maguire, B. C., Laxmikanthan, G., Schneider, C., Gaffney, B. J. and Brash, A. R. (2005a). On the Relationships of Substrate Orientation, Hydrogen Abstraction, and Product Stereochemistry in Single and Double Dioxygenations by Soybean Lipoxygenase-1 and Its Ala542Gly Mutant. J Biol Chem, 280, 38756-38766.

Coffa, G., Schneider, C. and Brash, A. R. (2005b). A comprehensive model of positional and stereo control in lipoxygenases. Biochem Biophys Res Commun, 338, 87-92.

Corbin, J. A., Evans, J. H., Landgraf, K. E. and Falke, J. J. (2007). Mechanism of specific membrane targeting by $\mathrm{C} 2$ domains: Localized pools of target lipids enhance $\mathrm{Ca}^{2+}$ affinity. Biochemistry, 46, 4322-4336.

Cotruvo Jr, J. A. and Stubbe, J. (2012). Metallation and mismetallation of iron and manganese proteins in vitro and in vivo: the class I ribonucleotide reductases as a case study. Metallomics, 4, 1020-1036.

Cristea, M., Engstrom, A., Su, C., Hornsten, L. and Oliw, E. H. (2005). Expression of manganese lipoxygenase in Pichia pastoris and site-directed mutagenesis of putative metal ligands. Arch Biochem Biophys, 434, 201-211.

Cui, W., Josyula, R., Li, J., Fu, Z. and Sha, B. (2011). Membrane Binding Mechanism of Yeast Mitochondrial Peripheral Membrane Protzein TIM44. Protein and peptide letters, 18, 718-725.

Dean, J. A. (1985). Lange's handbook of chemistry.

Di Venere, A., Salucci, M. L., van Zadelhoff, G., Veldink, G., Mei, G., Rosato, N., Finazzi-Agro, A. and Maccarrone, M. (2003). Structure-to-function relationship of minilipoxygenase, a 60-kDa fragment of soybean lipoxygenase-1 with lower stability but higher enzymatic activity. J Biol Chem, 278, 18281-18288.

Eek, P., Jarving, R., Jarving, I., Gilbert, N. C., Newcomer, M. E. and Samel, N. (2012). Structure of a calcium-dependent 11R-lipoxygenase suggests a mechanism for Ca2+-regulation. J Biol Chem.

Egmond, M. R., Vliegenthart, J. F. G. and Boldingh, J. (1972). Stereospecificity of the hydrogen abstraction at carbon atom n-8 in the oxygenation of linoleic acid by lipoxygenases from corn germs and soya beans. Biochem Biophys Res Commun, 48, 1055-1060. 
Emsley, P., Lohkamp, B., Scott, W. G. and Cowtan, K. (2010). Features and development of Coot. Acta Cryst, D66, 486-501.

Frankel, E. N., Neff, W. E., Rohwedder, W. K., Khambay, B. P. S., Garwood, R. F. and Weedon, B. C. L. (1977). Analysis of autoxidized fats by gas chromatography-mass spectrometry: II. methyl linoleate. Lipids, 12, 908-913.

Funk, C. D. (1996). The molecular biology of mammalian lipoxygenases and the quest for eicosanoid functions using lipoxygenase-deficient mice. Biochim Biophys Acta, 1304, 65-84.

Funk, C. D. (2001). Prostaglandins and leukotrienes: advances in eicosanoid biology. Science, 294, 1871-1875.

Furse, K. E., Pratt, D. A., Schneider, C., Brash, A. R., Porter, N. A. and Lybrand, T. P. (2006). Molecular dynamics simulations of arachidonic acid-derived pentadienyl radical intermediate complexes with COX-1 and COX-2: insights into oxygenation regio- and stereoselectivity. Biochemistry, 45, 3206-3218.

Gaffney, B. J., Mavrophilipos, D. V. and Doctor, K. S. (1993). Access of ligands to the ferric center in lipoxygenase-1. Biophys J, 64, 773.

Gao, B., Boeglin, W. E. and Brash, A. R. (2010). Omega-3 fatty acids are oxygenated at the n-7 carbon by the lipoxygenase domain of a fusion protein in the cyanobacterium Acaryochloris marina. Biochim Biophys Acta, 1801, 58-63.

Garreta, A., Val-Moraes, S. P., García-Fernández, Q., Busquets, M., Juan, C., Oliver, A. and other authors (2013). Structure and interaction with phospholipids of a prokaryotic lipoxygenase from Pseudomonas aeruginosa. FASEB J, 27, 4811-4821.

Gilbert, N. C., Bartlett, S. G., Waight, M. T., Neau, D. B., Boeglin, W. E., Brash, A. R. and Newcomer, M. E. (2011). The Structure of Human 5-Lipoxygenase. Science, 331, 217219.

Giles, N. M., Giles, G. I. and Jacob, C. (2003). Multiple roles of cysteine in biocatalysis. Biochem Biophys Res Commun, 300, 1-4.

Gill, S. C. and Von Hippel, P. H. (1989). Calculation of protein extinction coefficients from amino acid sequence data. Anal Biochem, 182, 319-326.

Gillmor, S. A., Villasenor, A., Fletterick, R., Sigal, E. and Browner, M. F. (1998). The structure of mammalian 15-lipoxygenase reveals similarity to the lipases and the determinants of substrate specificity. Nat Struct Biol, 4, 1003-1009.

Glickman, M. H. and Klinman, J. P. (1995). Nature of rate-limiting steps in the soybean lipoxygenase-1 reaction. Biochemistry, 34, 14077-14092.

Graff, G., Anderson, L. A. and Jaques, L. W. (1990). Preparation and purification of soybean lipoxygenase-derived unsaturated hydroperoxy and hydroxy fatty acids and determination of molar absorptivities of hydroxy fatty acids. Anal Biochem, 188, 38-47.

Graham, S. W., Olmstead, R. G. and Barrett, S. C. (2002). Rooting phylogenetic trees with distant outgroups: a case study from the commelinoid monocots. Mol Biol Evol, 19, 1769-1781.

Grimes, H. D., Tranbarger, T. J. and Franceschi, V. R. (1993). Expression and accumulation patterns of nitrogen-responsive lipoxygenase in soybeans. Plant Physiol, 103, 457-466.

Gu, L., Lajoie, C. and Kelly, C. (2003). Expression of a Phanerochaete chrysosporium manganese peroxidase gene in the yeast Pichia pastoris. Biotechnol Prog, 19, 14031409.

Hada, T., Swift, L. L. and Brash, A. R. (1997). Discovery of 5R-lipoxygenase activity in oocytes of the surf clam, Spisula solidissima. Biochim Biophys Acta, 1346, 109-119.

Haining, J. L. and Axelrod, B. (1958). Induction period in the lipoxidase-catalyzed oxidation of linoleic acid and its abolition by substrate peroxide. J Biol Chem, 232, 193-202.

Hamberg, M., Su, C. and Oliw, E. (1998). Manganese lipoxygenase - Discovery of a bis-allylic hydroperoxide as product and intermediate in a lipoxygenase reaction. $\mathrm{J}$ Biol Chem, $273,13080-13088$. 
Hammarberg, T., Provost, P., Persson, B. and Rådmark, O. (2000). The N-terminal domain of 5-lipoxygenase binds calcium and mediates calcium stimulation of enzyme activity. $J$ Biol Chem, 275, 38787-38793.

Hammel, M., Walther, M., Prassl, R. and Kühn, H. (2004). Structural flexibility of the Nterminal ß-barrel domain of 15 -lipoxygenase-1 probed by small angle $\mathrm{X}$-ray scattering. Functional consequences for activity regulation and membrane binding. J Mol Biol, 343, 917-929.

Hansen, J., Garreta, A., Benincasa, M., Fusté, M. C., Busquets, M. and Manresa, A. (2013). Bacterial lipoxygenases, a new subfamily of enzymes? A phylogenetic approach. Appl Microbiol Biotechnol, 97, 4737-4747.

Horn, T., Adel, S., Schumann, R., Sur, S., Kakularam, K. R., Polamarasetty, A., Redanna, P., Kühn, H. and Heydeck, D. (2015). Evolutionary aspects of lipoxygenases and genetic diversity of human leukotriene signaling. Prog Lipid Res, 57, 13-39.

Hornung, E., Walther, M., Kühn, H. and Feussner, I. (1999). Conversion of cucumber linoleate 13-lipoxygenase to a 9-lipoxygenating species by site-directed mutagenesis. Proc NatI Acad Sci USA, 96, 4192-4197.

Imlay, J. A. (2008). Cellular defenses against superoxide and hydrogen peroxide. Annu Rev Biochem, 77, 755.

Inoue, H., Nojima, H. and Okayama, H. (1990). High efficiency transformation of Escherichia coli with plasmids. Gene, 96, 23-28.

Ivanov, I., Heydeck, D., Hofheinz, K., Roffeis, J., O'Donnell, V. B., Kühn, H. and Walther, M. (2010). Molecular enzymology of lipoxygenases. Arch Biochem Biophys, 503, 161-174.

Ivanov, I., Di Venere, A., Horn, T., Scheerer, P., Nicolai, E., Stehling, S. and other authors (2011). Tight association of $\mathrm{N}$-terminal and catalytic subunits of rabbit 12/15lipoxygenase is important for protein stability and catalytic activity. Biochim Biophys Acta, 1811, 1001-1010.

Jones, G. D., Russell, L., Darley-Usmar, V. M., Stone, D. and Wilson, M. T. (1996). Role of lipid hydroperoxides in the activation of 15- lipoxygenase. Biochemistry, 35, 7197-7203.

Kabsch, W. (2010). XDS. Acta Cryst, D66, 125 - 132.

Kallio, J. P., Rouvinen, J., Kruus, K. and Hakulinen, N. (2011). Probing the Dioxygen Route in Melanocarpus albomyces Laccase with Pressurized Xenon Gas. Biochemistry, null-null.

Kearse, M., Moir, R., Wilson, A., Stones-Havas, S., Cheung, M., Sturrock, S. and other authors (2012). Geneious Basic: an integrated and extendable desktop software platform for the organization and analysis of sequence data. Bioinformatics, 28, 1647-1649.

Kitaguchi, H., Ohkubo, K., Ogo, S. and Fukuzumi, S. (2005). Direct ESR detection of pentadienyl radicals and peroxyl radicals in lipid peroxidation: mechanistic insight into regioselective oxygenation in lipoxygenases. J Am Chem Soc, 127, 6605-6609.

Knapp, M. J., Seebeck, F. P. and Klinman, J. P. (2001). Steric control of oxygenation regiochemistry in soybean lipoxygenase-1. J Am Chem Soc, 123, 2931-2932.

Knapp, M. J. and Klinman, J. P. (2003). Kinetic studies of oxygen reactivity in soybean lipoxygenase-1. Biochemistry, 42, 11466-11475.

Kobe, M. J., Neau, D. B., Mitchell, C. E., Bartlett, S. G. and Newcomer, M. E. (2014). The Structure of Human 15-Lipoxygenase-2 with a Substrate Mimic. J Biol Chem, 289, 85628569.

Koeduka, T., Kajiwara, T. and Matsui, K. (2007). Cloning of lipoxygenase genes from a cyanobacterium, Nostoc punctiforme, and its expression in Eschelichia coli. Curr Microbiol, 54, 315-319.

Kühn, H., Sprecher, H. and Brash, A. R. (1990). On singular or dual positional specificity of lipoxygenases. The number of chiral products varies with alignment of methylene groups at the active site of the enzyme. J Biol Chem, 265, 16300-16305.

Kühn, H. (2000). Structural basis for the positional specificity of lipoxygenases. Prostaglandins Other Lipid Mediat, 62, 255-270. 
Kühn, H., Saam, J., Eibach, S., Holzhütter, H.-G., Ivanov, I. and Walther, M. (2005). Structural biology of mammalian lipoxygenases: Enzymatic consequences of targeted alterations of the protein structure. Biochem Biophys Res Commun, 338, 93-101.

Kühn, H. and O'Donnell, V. B. (2006). Inflammation and immune regulation by $12 / 15$ lipoxygenases. Prog Lipid Res, 45, 334-356.

Kühn, H., Banthiya, S. and van Leyen, K. (2015). Mammalian lipoxygenases and their biological relevance. Biochim Biophys Acta, 1851, 308-330.

Kulkarni, S., Das, S., Funk, C. D., Murray, D. and Cho, W. (2002). Molecular Basis of the Specific Subcellular Localization of the C2-like Domain of 5-Lipoxygenase. J Biol Chem, 277, 13167-13174.

Laemmli, U. K. (1970). Cleavage of structural proteins during the assembly of the head of bacteriophage T4. Nature, 227, 680-684.

Lah, M. S., Dixon, M. M., Pattridge, K. A., Stallings, W. C., Fee, J. A. and Ludwig, M. L. (1995). Structure-function in Escherichia coli iron superoxide dismutase: comparisons with the manganese enzyme from Thermus thermophilus. Biochemistry, 34, 1646-1660.

Lang, I., Göbel, C., Porzel, A., Heilmann, I. and Feussner, I. (2008). A lipoxygenase with linoleate diol synthase activity from Nostoc sp. PCC 7120. Biochem J, 410, 347-357.

Lewis, E. R., Johansen, E. and Holman, T. R. (1999). Large competitive kinetic isotope effects in human 15-lipoxygenase catalysis measured by a novel HPLC method. J Am Chem Soc, 121, 1395-1396.

Li, Y. F., Heelis, P. F. and Sancar, A. (1991). Active site of DNA photolyase: tryptophan-306 is the intrinsic hydrogen atom donor essential for flavin radical photoreduction and DNA repair in vitro. Biochemistry, 30, 6322-6329.

Lõhelaid, H., Järving, R., Valmsen, K., Varvas, K., Kreen, M., Järving, I. and Samel, N. (2008). Identification of a functional allene oxide synthase-lipoxygenase fusion protein in the soft coral Gersemia fruticosa suggests the generality of this pathway in octocorals. Biochim Biophys Acta, 1780, 315-321.

Luna, V. M., Chen, Y., Fee, J. A. and Stout, C. D. (2008). Crystallographic studies of Xe and Kr binding within the large internal cavity of cytochrome ba(3) from Thermus thermophilus: Structural analysis and role of oxygen transport channels in the heme-Cu oxidases. Biochemistry, 47, 4657-4665.

Luna, V. M., Fee, J. A., Deniz, A. A. and Stout, C. D. (2012). Mobility of Xe atoms within the oxygen diffusion channel of cytochrome ba 3 oxidase. Biochemistry, 51, 4669-4676.

Maccarrone, M., Salucci, M. L., van Zadelhoff, G., Malatesta, F., Veldink, G., Vliegenthart, J. F. and Finazzi-Agro, A. (2001). Tryptic digestion of soybean lipoxygenase-1 generates a $60 \mathrm{kDa}$ fragment with improved activity and membrane binding ability. Biochemistry, 40, 6819-6827.

Mandel, M. and Higa, A. (1970). Calcium-dependent bacteriophage DNA infection. J Mol Biol, $53,159-162$.

Maret, W. (2010). Metalloproteomics, metalloproteomes, and the annotation of metalloproteins. Metallomics, 2, 117-125.

Marnett, L. J. (1987). Peroxyl free radicals: potential mediators of tumor initiation and promotion. Carcinogenesis, 8, 1365-1373.

Martin, J. E. and Imlay, J. A. (2011). The alternative aerobic ribonucleotide reductase of Escherichia coli, NrdEF, is a manganese-dependent enzyme that enables cell replication during periods of iron starvation. Mol Microbiol, 80, 319-334.

Martin, T. (1998). Phosphoinositide lipids as signaling molecules: common themes for signal transduction, cytoskeletal regulation, and membrane trafficking. Annu Rev Cell Dev Biol, 14, 231-264.

May, C., Höhne, M., Gnau, P., Schwennesen, K. and Kindl, H. (2000). The N-terminal $\beta$-barrel structure of lipid body lipoxygenase mediates its binding to liposomes and lipid bodies. Eur J Biochem, 267, 1100-1109. 
McCoy, A. J., Grosse-Kunstleve, R. W., Adams, P. D., Winn, M. D., Storoni, L. C. and Read, R. J. (2007). Phaser crystallographic software. J Appl Crystallogr, 40, 658-674.

Mereschkowsky, C. (1905). Über Natur und Ursprung der Chromatophoren im Pflanzenreiche.

Meyer, T. and Lamberts, B. (1965). Use of coomassie brilliant blue R250 for the electrophoresis of microgram quantities of parotid saliva proteins on acrylamide-gel strips. Biochim Biophys Acta, 107, 144-145.

Minor, W., Steczko, J., Stec, B., Otwinowski, Z., Bolin, J. T., Walter, R. and Axelrod, B. (1996). Crystal structure of soybean lipoxygenase L-1 at 1.4 A resolution. Biochemistry, 35, 10687-10701.

Moiseyev, N., Rucker, J. and Glickman, M. H. (1997). Reduction of ferric iron could drive hydrogen tunneling in lipoxygenase catalysis: Implications for enzymatic and chemical mechanisms. J Am Chem Soc, 119, 3853-3860.

Mortimer, M., Jarving, R., Brash, A. R., Samel, N. and Jarving, I. (2006). Identification and characterization of an arachidonate 11R-lipoxygenase. Arch Biochem Biophys, 445, 147-155.

Mullis, K., Faloona, F., Scharf, S., Saiki, R., Horn, G. and Erlich, H. (1992). Specific enzymatic amplification of DNA in vitro: the polymerase chain reaction. Biotechnology Series, 1717.

Murray, C. and Riley, J. (1969). The solubility of gases in distilled water and sea water-II. Oxygen. In Deep Sea Research and Oceanographic Abstracts, pp. 311-320: Elsevier.

Neau, D. B., Gilbert, N. C., Bartlett, S. G., Boeglin, W., Brash, A. R. and Newcomer, M. E. (2009). The $1.85 \mathrm{~A}$ structure of an $8 R$-lipoxygenase suggests a general model for lipoxygenase product specificity. Biochemistry, 48, 7906-7915.

Neau, D. B., Bender, G., Boeglin, W. E., Bartlett, S. G., Brash, A. R. and Newcomer, M. E. (2014). Crystal structure of a lipoxygenase in complex with substrate: the arachidonic acid-binding site of 8R-lipoxygenase. J Biol Chem, 289, 31905-31913.

Newcomer, M. E. and Brash, A. R. (2015). The structural basis for specificity in lipoxygenase catalysis. Protein Sci, 24, 298-309.

Newie, J., Andreou, A., Neumann, P., Einsle, O., Feussner, I. and Ficner, R. (2015). Crystal structure of a lipoxygenase from Cyanothece sp. may reveal novel features for substrate acquisition. J Lipid Res, doi: 10.1194/jlr.M064980.

Noverr, M. C., Toews, G. B. and Huffnagle, G. B. (2002). Production of prostaglandins and leukotrienes by pathogenic fungi. Infect Immun, 70, 400-402.

Oldham, M. L., Brash, A. R. and Newcomer, M. E. (2005). Insights from the X-ray crystal structure of coral 8R-lipoxygenase: calcium activation via a C2-like domain and a structural basis of product chirality. J Biol Chem, 280, 39545-39552.

Oliw, E. H., Cristea, M. and Hamberg, M. (2004). Biosynthesis and isomerization of 11hydroperoxylinoleates by manganese- and iron-dependent lipoxygenases. Lipids, 39, 319-323.

Oliw, E. H., Jernerén, F., Hoffmann, I., Sahlin, M. and Garscha, U. (2011). Manganese lipoxygenase oxidizes bis-allylic hydroperoxides and octadecenoic acids by different mechanisms. Biochim Biophys Acta, 1811, 138-147.

Pirrat, P., Smith, M. A., Pearson, A. R., McPherson, M. J. and Phillips, S. E. (2008). Structure of a xenon derivative of Escherichia coli copper amine oxidase: confirmation of the proposed oxygen-entry pathway. Acta Crystallogr Sect F Struct Biol Cryst Commun, 64, 1105-1109.

Porter, N. A. and Wujek, D. G. (1984). Autoxidation of polyunsaturated fatty acids, an expanded mechanistic study. J Am Chem Soc, 106, 2626-2629.

Porter, N. A. (1990). Autoxidation of polyunsaturated fatty acids: initiation, propagation, and product distribution (basic chemistry). In Membrane Lipid oxidation, pp. 33-62. Edited by C. Vigo-Pelfrey. Boca Raton: CRC Press.

Porter, N. A., Caldwell, S. E. and Mills, K. A. (1995). Mechanisms of free radical oxidation of unsaturated lipids. Lipids, 30, 277-290. 
Pratt, D. A., Mills, J. H. and Porter, N. A. (2003). Theoretical calculations of carbon-oxygen bond dissociation enthalpies of peroxyl radicals formed in the autoxidation of lipids. $J$ Am Chem Soc, 125, 5801-5810.

Regenstein, H. and Berndt, A. (1974). Homohyperconjugation in a perpendicular allyl radical. Angewandte Chemie International Edition in English, 13, 145-146.

Rickert, K. W. and Klinman, J. P. (1999). Nature of hydrogen transfer in soybean lipoxygenase 1: Separation of primary and secondary isotope effects. Biochemistry, 38, 1221812228.

Roschek, B., Tallman, K. A., Rector, C. L., Gillmore, J. G., Pratt, D. A., Punta, C. and Porter, N. A. (2006). Peroxyl radical clocks. J Org Chem, 71, 3527-3532.

Saam, J., Ivanov, I., Walther, M., Holzhütter, H.-G. and Kühn, H. (2007). Molecular dioxygen enters the active site of 12/15-lipoxygenase via dynamic oxygen access channels. Proc Natl Acad Sci USA, 104, 13319-13324.

Sambrook, J., Fritsch, E. F. and Maniatis, T. (1989). Molecular cloning: Cold spring harbor laboratory press New York.

Sanger, F., Nicklen, S. and Coulson, A. R. (1977). DNA sequencing with chain-terminating inhibitors. Proc Natl Acad Sci USA, 74, 5463-5467.

Saura, P., Suardíaz, R., Masgrau, L., Lluch, J. M. and González-Lafont, À. (2014). Unraveling how Enzymes Can Use Bulky Residues to Drive Site-Selective C-H Activation: The Case of Mammalian Lipoxygenases Catalyzing Arachidonic Acid Oxidation. ACS Catalysis.

Schilstra, M. J., Veldink, G. A., Verhagen, J. and Vliegenthart, J. F. G. (1992). Effect of Lipid Hydroperoxide on Lipoxygenase Kinetics. Biochemistry, 31, 7692-7699.

Schilstra, M. J., Veldink, G. A. and Vliegenthart, J. F. (1993). Kinetic analysis of the induction period in lipoxygenase catalysis. Biochemistry, 32, 7686-7691.

Schneider, C., Keeney, D. S., Boeglin, W. E. and Brash, A. R. (2001). Detection and cellular localization of $12 R$-lipoxygenase in human tonsils. Arch Biochem Biophys, 386, 268274.

Schneider, C., Pratt, D. A., Porter, N. A. and Brash, A. R. (2007). Control of oxygenation in lipoxygenase and cyclooxygenase catalysis. Chem Biol, 14, 473-488.

Schreiner, K. and Berndt, A. (1974). ESR-Spektrum eines um $90^{\circ}$ verdrillten Benzyl-Radikals. Angew Chem, 86, 131-132.

Schrödinger, L. The PyMOL molecular graphics system. 2010. Version, 1, r1.

Schwarz, K., Borngräber, S., Anton, M. and Kühn, H. (1998). Probing the substrate alignment at the active site of 15-lipoxygenases by targeted substrate modification and sitedirected mutagenesis. Evidence for an inverse substrate orientation. Biochemistry, 37, 15327-15335.

Segraves, E. N., Chruszcz, M., Neidig, M. L., Ruddat, V., Zhou, J., Wecksler, A. T., Minor, W., Solomon, E. I. and Holman, T. R. (2006). Kinetic, Spectroscopic, and Structural Investigations of the Soybean Lipoxygenase-1 First-Coordination Sphere Mutant, Asn694Gly. Biochemistry, 45, 10233-10242.

Senger, T., Wichard, T., Kunze, S., Göbel, C., Lerchl, J., Pohnert, G. and Feussner, I. (2005). A multifunctional lipoxygenase with fatty acid hydroperoxide cleaving activity from the moss Physcomitrella patens. J Biol Chem, 280, 7588-7596.

Shimizu, T. (2009). Lipid mediators in health and disease: enzymes and receptors as therapeutic targets for the regulation of immunity and inflammation. Annu Rev Pharmacol Toxicol, 49, 123-150.

Skrzypczak-Jankun, E., Bross, R. A., Carroll, R. T., Dunham, W. R. and Funk, M. O., Jr. (2001). Three-dimensional structure of a purple lipoxygenase. J Am Chem Soc, 123, 1081410820.

Slappendel, S., Veldink, G. A., Vliegenthart, J. F., Aasa, R. and Malmstrom, B. G. (1981). EPR spectroscopy of soybean lipoxygenase-1. Description and quantification of the highspin fe(III) signals. Biochim Biophys Acta, 667, 77-86. 
Sloane, D. L., Leung, R., Craik, C. S. and Sigal, E. (1991). A primary determinant for lipoxygenase positional specificity. Nature, 354, 149-152.

Smith, W. L. and Lands, W. E. (1972). Oxygenation of unsaturated fatty acids by soybean lipoxygenase. J Biol Chem, 247, 1038-1047.

Sobota, J. M. and Imlay, J. A. (2011). Iron enzyme ribulose-5-phosphate 3-epimerase in Escherichia coli is rapidly damaged by hydrogen peroxide but can be protected by manganese. Proc Natl Acad Sci USA, 108, 5402-5407.

Steinhilber, D. (1999). 5-Lipoxygenase: a target for antiinflammatory drugs revisited. Curr Med Chem, 6, 71-85.

Studier, F. W. (2005). Protein production by auto-induction in high density shaking cultures. Protein Expr Purif, 41, 207-234.

Su, C. and Oliw, E. H. (1998). Manganese lipoxygenase - Purification and characterization. J Biol Chem, 273, 13072-13079.

Su, C., Sahlin, M. and Oliw, E. H. (2000). Kinetics of manganese lipoxygenase with a catalytic mononuclear redox center. J Biol Chem, 275, 18830-18835.

Suardíaz, R., Masgrau, L., Lluch, J. M. and González-Lafont, À. (2013). On the regio- and stereospecificity of arachidonic acid peroxidation catalyzed by mammalian 15lypoxygenases: a combined molecular dynamics and QM/MM study. Chemphyschem, 14, 3777-3787.

Suardíaz, R., Masgrau, L., Lluch, J. M. and González-Lafont, À. (2014). Regio- and stereospecificity in the oxygenation of arachidonic acid catalyzed by Leu597 mutants of rabbit 15-lipoxygenase: a QM/MM study. Chemphyschem, 15, 2303-2310.

Tallman, K. A., Pratt, D. A. and Porter, N. A. (2001). Kinetic products of linoleate peroxidation: rapid beta-fragmentation of nonconjugated peroxyls. J Am Chem Soc, 123, 1182711828.

Tatulian, S. A., Steczko, J. and Minor, W. (1998). Uncovering a calcium-regulated membranebinding mechanism for soybean lipoxygenase-1. Biochemistry, 37, 15481-15490.

Tomchick, D. R., Phan, P., Cymborowski, M., Minor, W. and Holman, T. R. (2001). Structural and functional characterization of second-coordination sphere mutants of soybean lipoxygenase-1. Biochemistry, 40, 7509-7517.

Tranbarger, T. J., Franceschi, V. R., Hildebrand, D. F. and Grimes, H. D. (1991). The soybean 94-kilodalton vegetative storage protein is a lipoxygenase that is localized in paraveinal mesophyll cell vacuoles. Plant Cell, 3, 973-987.

Trott, O. and Olson, A. J. (2010). AutoDock Vina: improving the speed and accuracy of docking with a new scoring function, efficient optimization, and multithreading. Journal of computational chemistry, 31, 455-461.

Tsai, A. L., Palmer, G., Wu, G., Peng, S., Okeley, N. M., van der Donk, W. A. and Kulmacz, R. J. (2002). Structural characterization of arachidonyl radicals formed by aspirin-treated prostaglandin $\mathrm{H}$ synthase-2. J Biol Chem, 277, 38311-38321.

Tsitsigiannis, D. I. and Keller, N. P. (2007). Oxylipins as developmental and host-fungal communication signals. Trends Microbiol, 15, 109-118.

Tu, W. Y., Pohl, S., Gray, J., Robinson, N. J., Harwood, C. R. and Waldron, K. J. (2012). Cellular iron distribution in Bacillus anthracis. J Bacteriol, 194, 932-940.

van Overveld, F. W., Haenen, G. R., Rhemrev, J., Vermeiden, J. P. and Bast, A. (2000). Tyrosine as important contributor to the antioxidant capacity of seminal plasma. Chemico-biological interactions, 127, 151-161.

Walker, J. M. (2005). The proteomics protocols handbook. Totowa, N.J.: Humana Press.

Walther, M., Anton, M., Wiedmann, M., Fletterick, R. and Kühn, H. (2002). The N-terminal domain of the reticulocyte-type 15-lipoxygenase is not essential for enzymatic activity but contains determinants for membrane binding. J Biol Chem, 277, 27360-27366.

Walther, M., Hofheinz, K., Vogel, R., Roffeis, J. and Kühn, H. (2011). The N-terminal $\beta$-barrel domain of mammalian lipoxygenases including mouse 5-lipoxygenase is not essential 
for catalytic activity and membrane binding but exhibits regulatory functions. Arch Biochem Biophys, 516, 1-9.

Wang, X., Devaiah, S. P., Zhang, W. and Welti, R. (2006). Signaling functions of phosphatidic acid. Prog Lipid Res, 45, 250-278.

Weiss, B. and Richardson, C. C. (1967). Enzymatic breakage and joining of deoxyribonucleic acid, I. Repair of single-strand breaks in DNA by an enzyme system from Escherichia coli infected with T4 bacteriophage. Proc Natl Acad Sci USA, 57, 1021.

Wennman, A., Jerneren, F., Hamberg, M. and Oliw, E. H. (2012). Catalytic convergence of manganese and iron lipoxygenases by replacement of a single amino acid. J Biol Chem, 287, 31757-31765.

Wennman, A. and Oliw, E. H. (2013). Secretion of two novel enzymes, manganese 9Slipoxygenase and epoxy alcohol synthase, by the rice pathogen Magnaporthe salvinii. $J$ Lipid Res, 54, 762-775.

Wennman, A., Magnuson, A., Hamberg, M. and Oliw, E. H. (2015). Manganese lipoxygenase of Fusarium oxysporum and the structural basis for biosynthesis of distinct 11hydroperoxy stereoisomers. J Lipid Res.

Winn, M. D., Ballard, C. C., Cowtan, K. D., Dodson, E. J., Emsley, P., Evans, P. R. and other authors (2011). Overview of the CCP4 suite and current developments. Acta Crystallogr D Biol Crystallogr, 67, 235-242.

Woodman, M. E. (2008). Direct PCR of intact bacteria (colony PCR). Curr Protoc Microbiol, Appendix 3, Appendix 3D.

Wright, J. S., Shadnia, H. and Chepelev, L. L. (2009). Stability of carbon-centered radicals: Effect of functional groups on the energetics of addition of molecular oxygen. $J$ Comput Chem, 30, 1016-1026.

Xu, S., Mueser, T. C., Marnett, L. J. and Funk, M. O. (2012). Crystal Structure of 12Lipoxygenase Catalytic-Domain-Inhibitor Complex Identifies a Substrate-Binding Channel for Catalysis. Structure, 20, 1490-1497.

Xue, H., Chen, X. and Li, G. (2007). Involvement of phospholipid signaling in plant growth and hormone effects. Curr Opin Plant Biol, 10, 483-489.

Yin, H. and Porter, N. (2005). New insights regarding the autoxidation of polyunsaturated Fatty acids. Antioxid Redox Signal, 7, 170-184.

Zhang, C., Tao, T., Ying, Q., Zhang, D., Lu, F., Bie, X. and Lu, Z. (2012). Extracellular production of lipoxygenase from Anabaena sp. PCC 7120 in Bacillus subtilis and its effect on wheat protein. Appl Microbiol Biotechnol, 94, 949-958.

Zheng, Y., Boeglin, W. E., Schneider, C. and Brash, A. R. (2008). A 49-kDa Mini-lipoxygenase from Anabaena sp. PCC 7120 retains catalytically complete functionality. J Biol Chem, 283, 5138-5147. 


\section{List of abbreviations}

According to the IUPAC-IUB Commission, the three-letter code was used for amino acids.

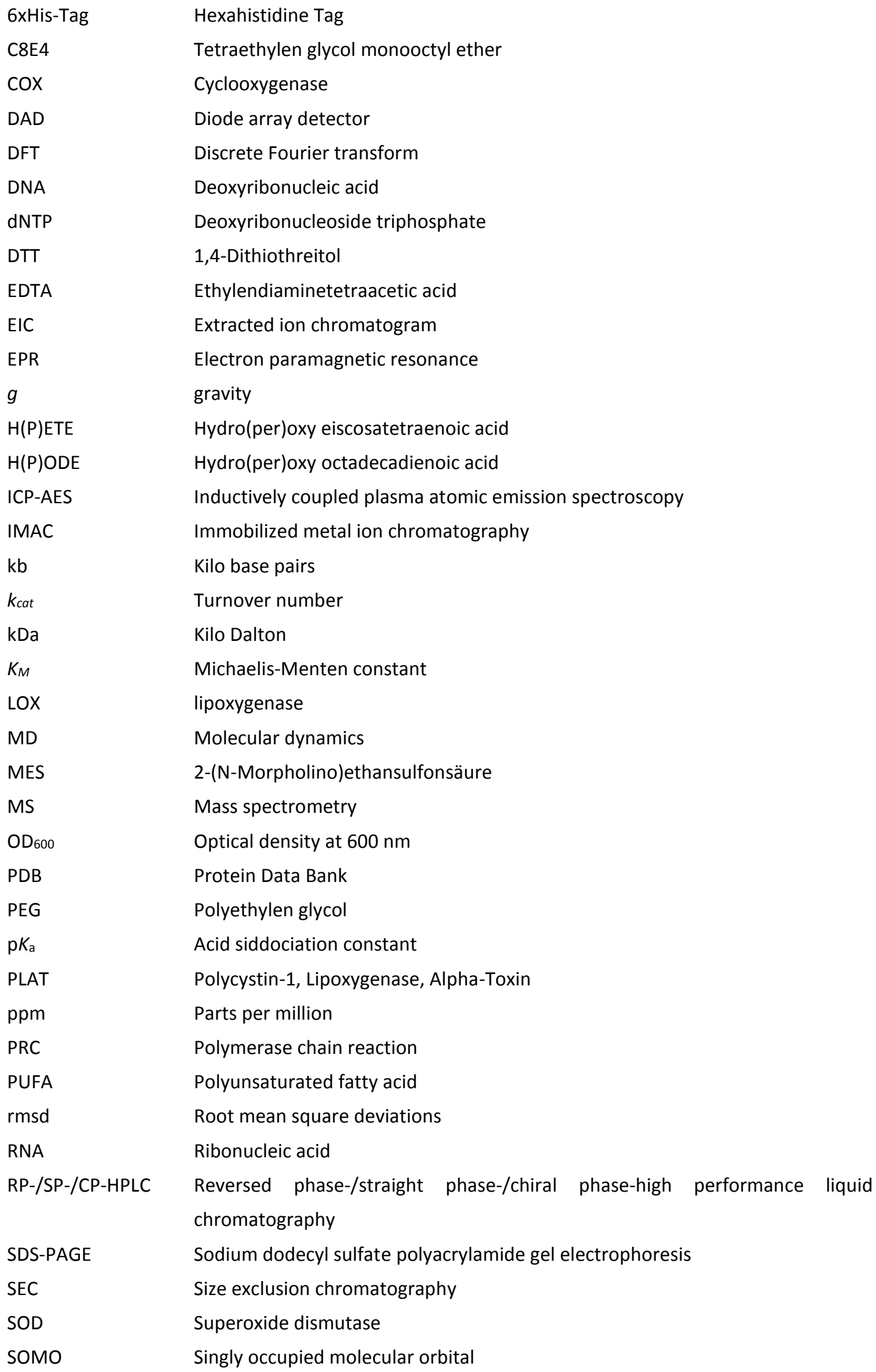


sp.

TAE

TEMED

TIC

UHPLC-MS

UV

$\mathrm{v} / \mathrm{v}$

vis

w/o

$\mathrm{w} / \mathrm{v}$

wt species

Tris-acetate-EDTA

Tetramethylethylenediamine

Total ion chromatogram

Ultra-high performance liquid chromatography coupled to mass spectrometry Ultra violet (light)

Volume per volume

Visible light

without

Weight per voliume

Wild type 


\section{Supplements}

A
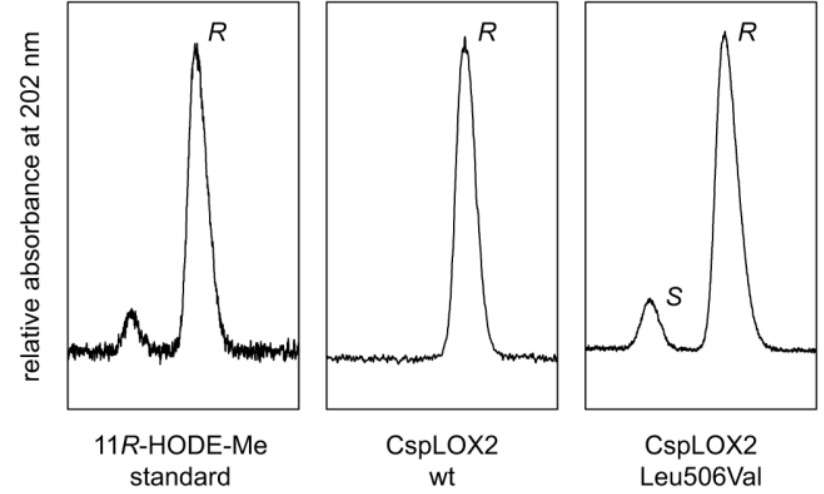

B
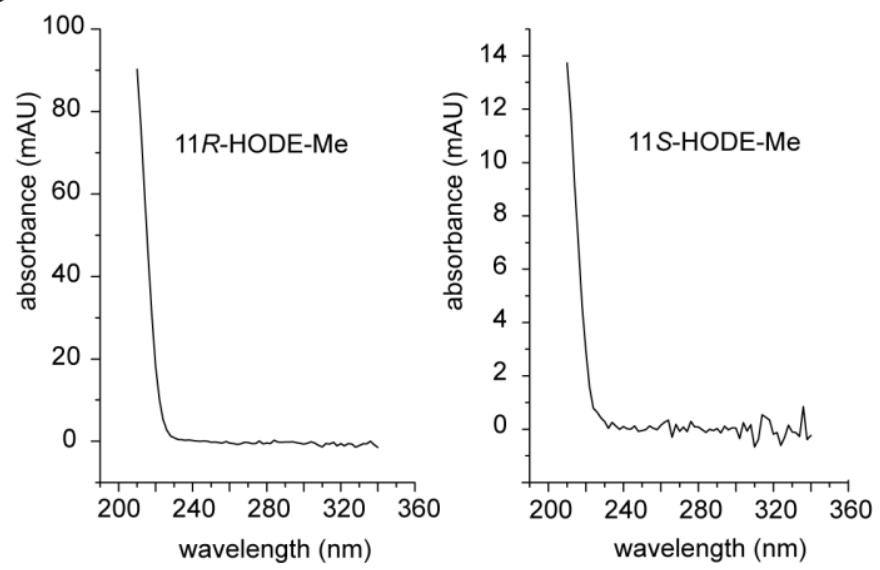

C

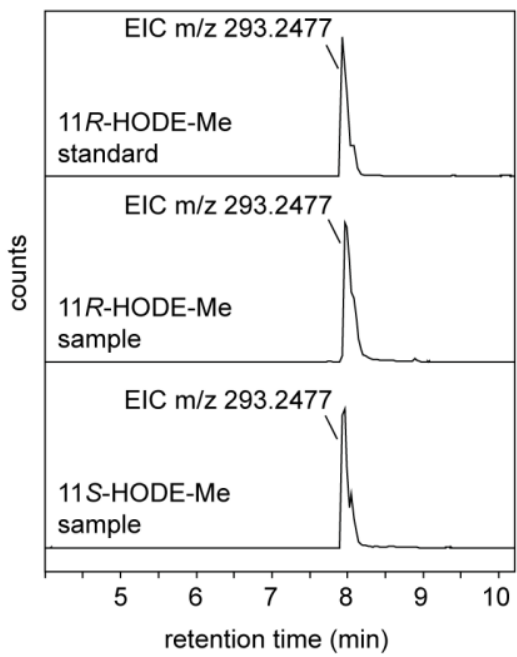

D

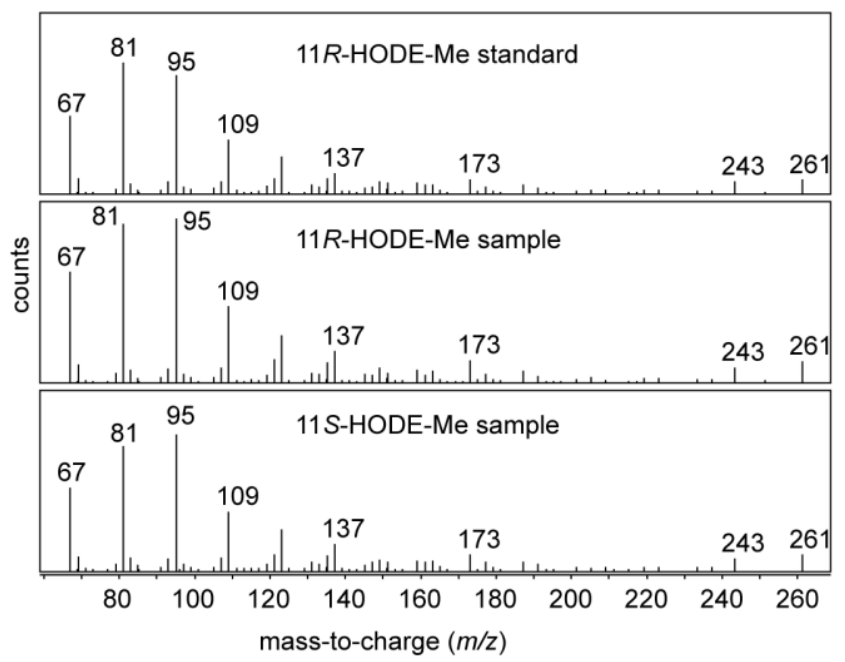

Supplemental Figure 1. Stereospecificity of the bis-allylic product from linoleic acid. The HPODEs formed by CspLOX2 wt and its variant Leu506Val were reduced to hydroxides and derivatized to their methyl esters (HODE-Me). (A) The $11 R$-HODE-Me standard as well as the HODE-Me of the CspLOX2 wt and its Leu506Val variant were separated by CPHPLC at a flow rate of $0.3 \mathrm{ml} / \mathrm{min}$. A small peak eluting before the $11 R$-HODE-Me was suspected to be the $\mathrm{S}$ enantiomer of the compound, since both compounds show a very similar UV-vis spectrum (B). To confirm the identity of 11S-HODE-Me, the 11R-HODE-Me peaks of the standard and CspLOX2 wt as well as the putative 11S-HODE-Me peak were purified by CP-HPLC and subjected to high resolution LC MS/MS. The extracted ion chromatograms for the ion $\left[\mathrm{M}-\mathrm{H}_{2} \mathrm{O}+\mathrm{H}\right]^{+}$at $\mathrm{m} / \mathrm{z}=293.2477$ (C) and the high resolution mass spectra (mass resolution $<2 \mathrm{ppm}$ ) of these samples were compared. As retention time (C) and mass spectra (D) are identical, both compounds are probably 11-HODE-Me and only differ in their stereochemstry. 

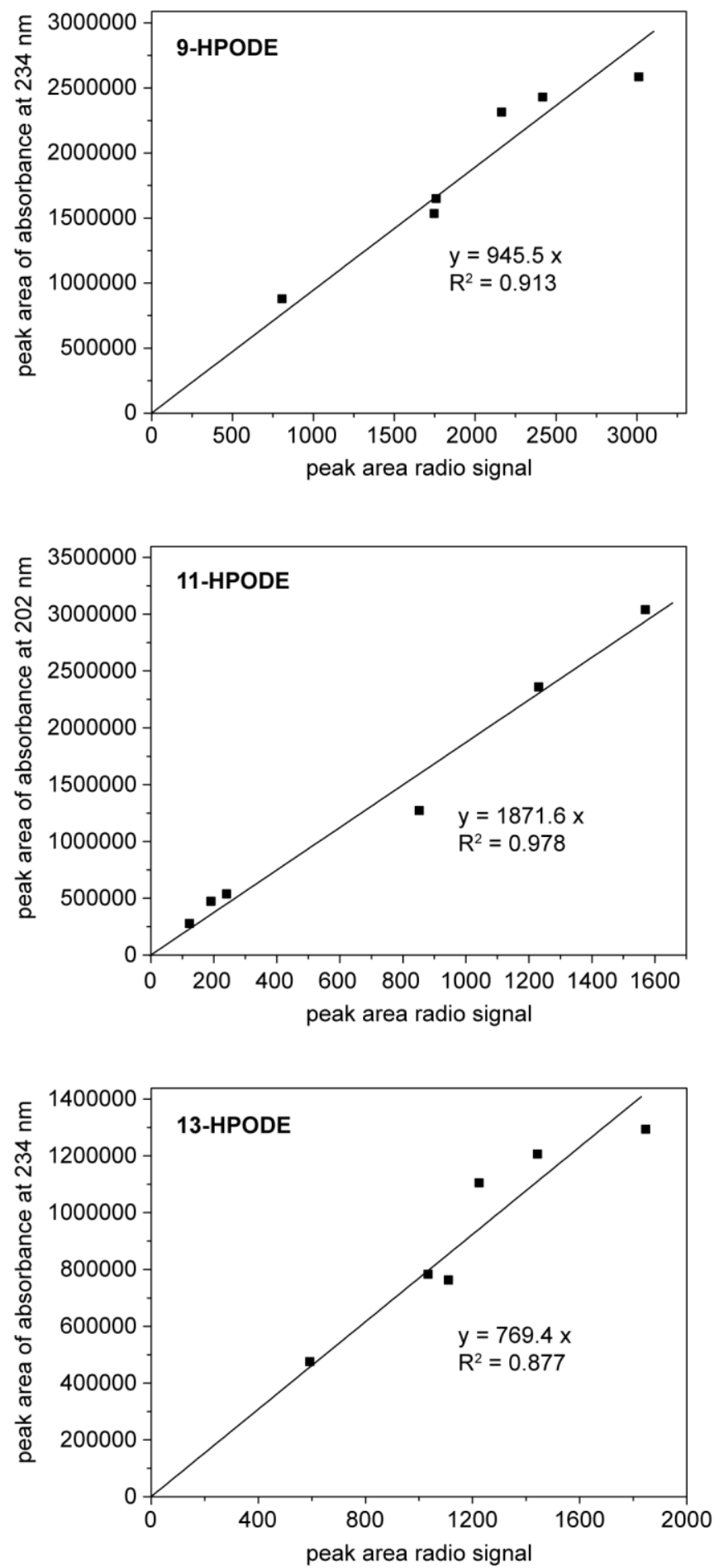

Supplemental Figure 2. Ratio of radio vs. absorbance signal of different HPODEs. Different amounts of reaction product were subjected to radio-RP-HPLC analysis. The resulting peak areas of the absorbance at $202 \mathrm{~nm}$ (11-HPODE) or $234 \mathrm{~nm}$ (9- or 13-HPODE) were plotted against the peak area of the radio signal of the same peak. From the slopes of the linear regression curves the following scaling factors for the individual HPODEs were determined: 0.791 for 9HPODE, 1.566 for 11-HPODE and 0.644 for 13-HPODE. 


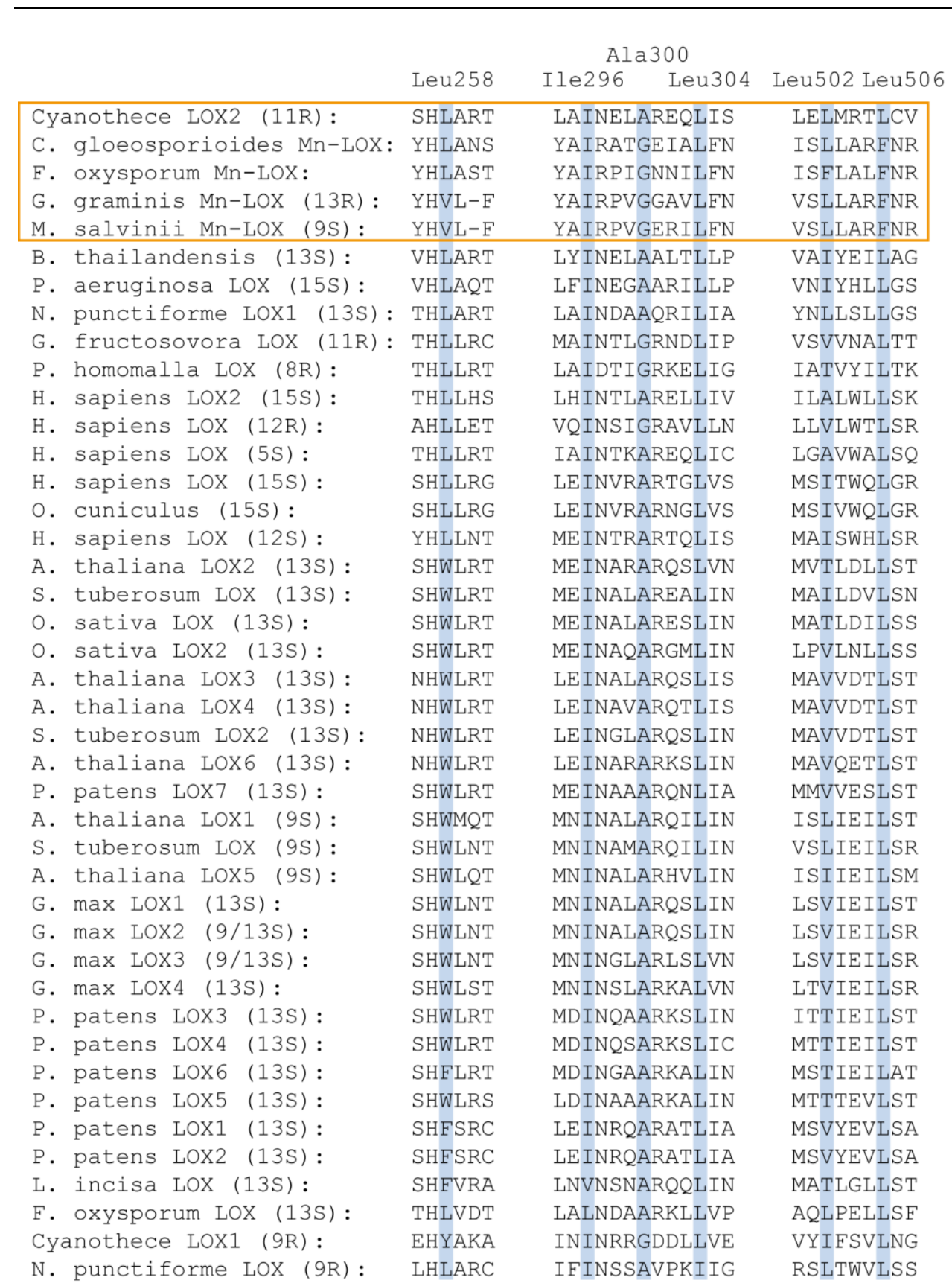

Supplemental Figure 3. Sequence alignments of the active site clamp residues identified in CspLOX2 with other LOXs that were also used for the phylogenetic tree in Figure 15. See this figure legend for respective accession numbers. The crucial amino acids of the active site are highlighted in blue. The orange box frames the LOXs that can form bisallylic products in significant amounts. 


\begin{tabular}{|c|c|c|c|}
\hline Cyanothece Lox2 (11R): & ISHLARTHLI & PQHAAVNYP & STSI \\
\hline C. gloeosporioides Mn-LOX: & IYHLANSHAV & VSHHVLNQG & YLSV \\
\hline F. oxysporum Mn-LOX: & MYHLASTHAV & VSHHVLNQG & YLSV \\
\hline G. graminis Mn-LOX (13R): & MYHVL-FHTI & GAHHVMNQG & FLSV \\
\hline M. salvinii Mn-LOX (9S): & MYHVL-FHTV & GAHHVLNQG & \\
\hline B. thailandensis (13S): & FVHLARTHLV & AQHAAVNES & $\mathrm{NI}$ \\
\hline P. aeruginosa LOX (15S): & FVHLAQTHLV & AQHAAVNEP & I \\
\hline N. punctiforme LOX1 (13S): & VTHLARTHLL & AQHAAVNEP & NI \\
\hline G. fructosovora LOX (11R): & ITHLLRCHLM & CQHAAVNFS & IAI \\
\hline P. homomalla LOX (8R): & NTHLLRTHLT & CQHAAVNFS & TAI \\
\hline H. sapiens LOX2 (15S): & LTHLLHSHLL & AKHAAVSAG & VSI \\
\hline H. sapiens LOX (12R): & IAHLLETHLI & AKHAAVNTG & ISI \\
\hline H. sapiens LOX (5S): & ITHLLRTHLV & AQHAAVNFG & VAI \\
\hline H. sapiens LOX (15S): & QSHLLRGHLM & GQHASVHLG & VAI \\
\hline O. cuniculus (15S): & NSHLLRGHLM & GQHSS IHLG & JAI \\
\hline H. sapiens LOX (12S): & QYHLLNTHLV & AQHAAINQG & VTI \\
\hline A. thaliana Lox2 (13S): & ISHWLRTHAC & GHHAAVNFG & ISI \\
\hline S. tuberosum LOX (13S): & VSHWLRTHCC & GHHAAVNFG & ISI \\
\hline O. sativa LOX (13S): & VSHWLRTHCC & GHHAAVNFG & ISI \\
\hline O. sativa LOX2 (13S): & VSHWLRTHCC & GHHAAVNFG & I \\
\hline A. thaliana Lox3 (13S): & VNHWLRTHAC & AQHAALNFG & VSI \\
\hline A. thaliana LOX4 (13S): & VNHWLRTHAC & AQHAALNFG & VSI \\
\hline S. tuberosum LOX2 (13S): & VNHWLRTHAS & AQHAALNFG & SI \\
\hline A. thaliana Lox6 (13S): & VNHWLRTHAS & GQHAAINFG & ISI \\
\hline P. patens LOX7 (13S): & VSHWLRTHAC & GHHAAVNFG & ISI \\
\hline A. thaliana Lox1 (9S): & ISHWMQTHAS & ALHAAVNFG & VSI \\
\hline S. tuberosum LOX (9S): & ISHWLNTHAV & ALHAAVNFG & SVSI \\
\hline A. thaliana Lox5 (9S): & ISHWLQTHAV & ALHAAVNFG & SVSI \\
\hline G. $\max$ LOX1 (13S): & MSHWLNTHAA & ALHAAVNFG & SISI \\
\hline G. $\max \operatorname{Lox} 2$ (9/13S) : & MSHWLNTHAV & ALHAAVNFG & SISI \\
\hline G. $\max \operatorname{Lox} 3(9 / 13 S)$ : & VSHWLNTHAV & ALHAAVNFG & ISI \\
\hline G. $\max$ LOX4 (13S) : & ISHWLSTHAI & ALHAAVNFG & SISI \\
\hline P. patens LOX3 (13S): & ISHWLRTHAA & CHHAAVNFG & SVSI \\
\hline P. patens LOX4 (13S): & ISHWLRTHAV & CHHAAVNFG & SISI \\
\hline P. patens LOX6 (13S): & ISHFLRTHAC & CHHAAVNFG & SISI \\
\hline P. patens LOX5 (13S): & SSHWLRSHAT & CHHAAVNFG & STSI \\
\hline P. patens LOX1 (13S): & FSHFSRCHAV & CLHAAVNFG & SITI \\
\hline P. patens LOX2 (13S): & FSHFSRCHAV & CLHAAVNFG & SITI \\
\hline F. oxysporum LOX (13S): & ATHLVDTHMI & PQHTAVNYL & SLI \\
\hline Cyanothece Lox1 (9R): & IEHYAKAHVQ & LWHTWVNDS & LINI \\
\hline N. punctiforme LOX (9R) & KLHLARCHFN & FLHSWANFK & \\
\hline
\end{tabular}

Supplemental Figure 4. Sequence alignment of the metal ligands in different LOXs that were also used for the phylogenetic tree in Figure 15. See this figure legend for respective accession numbers. 
Supplemental Table 1. List of all plant LOX structures deposited in the PDB. Four different enzymes are found from soybean: soybean LOX1, LOX2, LOXB and LOXD (plus their derivatives).

\begin{tabular}{|c|c|c|c|c|c|}
\hline Code & $\begin{array}{l}\text { Year of } \\
\text { release }\end{array}$ & First author & Organism/Enzyme & $\begin{array}{l}\text { Resolution } \\
(\AA)\end{array}$ & Title \\
\hline $2 S B L$ & 1995 & Amzel & Soybean LOX-1 & 2.6 & $\begin{array}{l}\text { THE THREE-DIMENSIONAL STRUCTURE } \\
\text { OF AN ARACHIDONIC ACID 15- } \\
\text { LIPOXYGENASE }\end{array}$ \\
\hline 1YGE & 1997 & Minor & Soybean LOX-1 & 1.4 & LIPOXYGENASE-1 (SOYBEAN) AT 100K \\
\hline 4WHA & 2014 & Scouras & Soybean LOX-1 & 1.7 & $\begin{array}{l}\text { Lipoxygenase-1 (soybean) } \\
\text { L546A/L754A mutant }\end{array}$ \\
\hline $1 \mathrm{~F} 8 \mathrm{~N}$ & 2001 & Tomchick & Soybean LOX-1 & 1.4 & $\begin{array}{l}\text { LIPOXYGENASE-1 (SOYBEAN) AT 100K, } \\
\text { NEW REFINEMENT }\end{array}$ \\
\hline 3BNB & 2008 & Tomchick & Soybean LOX-1 & 1.45 & $\begin{array}{l}\text { Lipoxygenase-1 (Soybean) I553L } \\
\text { Mutant }\end{array}$ \\
\hline $3 B N C$ & 2008 & Tomchick & Soybean LOX-1 & 1.65 & $\begin{array}{l}\text { Lipoxygenase-1 (Soybean) I553G } \\
\text { Mutant }\end{array}$ \\
\hline 3BND & 2008 & Tomchick & Soybean LOX-1 & 1.6 & $\begin{array}{l}\text { Lipoxygenase-1 (Soybean), I553V } \\
\text { Mutant }\end{array}$ \\
\hline 3BNE & 2008 & Tomchick & Soybean LOX-1 & 1.4 & $\begin{array}{l}\text { Lipoxygenase-1 (Soybean) I553A } \\
\text { Mutant }\end{array}$ \\
\hline 1Y4K & 2005 & Chruszcz & Soybean LOX-1 & 1.95 & $\begin{array}{l}\text { Lipoxygenase-1 (Soybean) at 100K, } \\
\text { N694G Mutant }\end{array}$ \\
\hline 1FGM & 2001 & Tomchick & Soybean LOX-1 & 1.9 & $\begin{array}{l}\text { LIPOXYGENASE-1 (SOYBEAN) AT 100K, } \\
\text { N694H MUTANT }\end{array}$ \\
\hline $1 F G O$ & 2001 & Tomchick & Soybean LOX-1 & 1.62 & $\begin{array}{l}\text { LIPOXYGENASE-1 (SOYBEAN) AT 100K, } \\
\text { Q495A MUTANT }\end{array}$ \\
\hline $1 F G Q$ & 2001 & Tomchick & Soybean LOX-1 & 1.85 & $\begin{array}{l}\text { LIPOXYGENASE-1 (SOYBEAN) AT 100K, } \\
\text { Q495E MUTANT }\end{array}$ \\
\hline $1 F G R$ & 2001 & Tomchick & Soybean LOX-1 & 1.6 & $\begin{array}{l}\text { LIPOXYGENASE-1 (SOYBEAN) AT 100K, } \\
\text { Q697E MUTANT }\end{array}$ \\
\hline 3PZW & 2011 & Chruszcz & Soybean LOX-1 & 1.4 & $\begin{array}{l}\text { Soybean lipoxygenase-1 - re- } \\
\text { refinement }\end{array}$ \\
\hline 4WHA & 2014 & Scouras & Soybean LOX-1 & 1.7 & $\begin{array}{l}\text { Lipoxygenase-1 (soybean) } \\
\text { L546A/L754A mutant }\end{array}$ \\
\hline $4 W F O$ & 2015 & Carr & Soybean LOX-1 & 1.14 & $\begin{array}{l}\text { manganese-substituted soybean } \\
\text { lipoxygenase-1 }\end{array}$ \\
\hline 5EEO & 2015 & Mikami & Soybean LOX-1 & 2.1 & soybean lipoxygenase(L1)-T756R \\
\hline $1 \mathrm{HU9}$ & 2003 & Zhou & Soybean LOX-3 & 2.2 & $\begin{array}{l}\text { LIPOXYGENASE-3 (SOYBEAN) COMPLEX } \\
\text { WITH 4-HYDROPEROXY-2-METHOXY- } \\
\text { PHENOL }\end{array}$ \\
\hline $1 \mathrm{LNH}$ & 1997 & $\begin{array}{l}\text { Skrzypczak- } \\
\text { Jankun }\end{array}$ & Soybean LOX-3 & 2.6 & $\begin{array}{l}\text { LIPOXYGENASE-3(SOYBEAN) NON- } \\
\text { HEME FE(II) METALLOPROTEIN }\end{array}$ \\
\hline $1 \mathrm{JNQ}$ & 2003 & Zhou & Soybean LOX-3 & 2.1 & $\begin{array}{l}\text { LIPOXYGENASE-3 (SOYBEAN) COMPLEX } \\
\text { WITH EPIGALLOCATHECHIN (EGC) }\end{array}$ \\
\hline $1 R R L$ & 2004 & Borbulevych & Soybean LOX-3 & 2.09 & $\begin{array}{l}\text { Soybean Lipoxygenase (LOX-3) at } 93 \mathrm{~K} \\
\text { at 2.0 A resolution }\end{array}$ \\
\hline $1 \mathrm{RRH}$ & 2004 & Borbulevych & Soybean LOX-3 & 2.00 & $\begin{array}{l}\text { Soybean Lipoxygenase (LOX-3) at } \\
\text { ambient temperatures at } 2.0 \mathrm{~A} \\
\text { resolution }\end{array}$ \\
\hline
\end{tabular}


Supplemental Table 2. List of all bacterial LOX structures deposited in the PDB. Only the Pseudomonas LOX has been crystalized in different forms.

\begin{tabular}{|c|c|c|c|c|c|}
\hline Code & $\begin{array}{l}\text { Year of } \\
\text { release }\end{array}$ & First author & Organism/Enzyme & $\begin{array}{l}\text { Resolution } \\
(\AA)\end{array}$ & Title \\
\hline $4 \mathrm{G} 32$ & 2013 & Carpena & $\begin{array}{l}\text { Pseudomonas } \\
\text { aeruginosa LOX }\end{array}$ & 1.75 & $\begin{array}{l}\text { Crystal Structure of a Phospholipid- } \\
\text { Lipoxygenase Complex from } \\
\text { Pseudomonas aeruginosa at 1.75A } \\
\text { (P21212) }\end{array}$ \\
\hline $4 \mathrm{G} 33$ & 2013 & Carpena & $\begin{array}{l}\text { Pseudomonas } \\
\text { aeruginosa LOX }\end{array}$ & 2.0 & $\begin{array}{l}\text { Crystal Structure of a Phospholipid- } \\
\text { Lipoxygenase Complex from } \\
\text { Pseudomonas aeruginosa at } 2.0 \mathrm{~A} \\
\text { (C2221) }\end{array}$ \\
\hline 4RPE & 2015 & Carpena & $\begin{array}{l}\text { Pseudomonas } \\
\text { aeruginosa LOX }\end{array}$ & 1.6 & $\begin{array}{l}\text { Crystal Structure of Variant G186E } \\
\text { from Pseudomonas Aeruginosa } \\
\text { Lipoxygenase } 2 \text { at } 1.60 \mathrm{~A}(\mathrm{C} 2)\end{array}$ \\
\hline
\end{tabular}

Supplemental Table 3. List of all coral LOX structures deposited in the PDB. Structures of the Plexaura homomalla 8RLOX and Germesia fruticosa $11 R$-LOX are available.

\begin{tabular}{|c|c|c|c|c|c|}
\hline Code & $\begin{array}{l}\text { Year of } \\
\text { release }\end{array}$ & First author & Organism/Enzyme & $\begin{array}{l}\text { Resolution } \\
(\AA)\end{array}$ & Title \\
\hline 4QWT & 2014 & Neau & $\begin{array}{l}\text { Plexaura } \\
\text { homomalla } \\
8 R \text {-LOX }\end{array}$ & 2.0 & $\begin{array}{l}\text { Anaerobic crystal structure of } \\
\text { delta413-417:GS LOX in complex with } \\
\text { arachidonate }\end{array}$ \\
\hline $2 \mathrm{FNQ}$ & 2006 & Oldham & $\begin{array}{l}\text { Plexaura } \\
\text { homomalla } \\
8 R \text {-LOX }\end{array}$ & 3.2 & $\begin{array}{l}\text { Insights from the X-ray crystal } \\
\text { structure of coral 8R-lipoxygenase: } \\
\text { calcium activation via A C2-like domain } \\
\text { and a structural basis of product } \\
\text { chirality }\end{array}$ \\
\hline $3 F G 3$ & 2009 & Neau & $\begin{array}{l}\text { Plexaura } \\
\text { homomalla } \\
8 R \text {-LOX }\end{array}$ & 1.9 & $\begin{array}{l}\text { Crystal structure of Delta413-417:GS } \\
\text { I805W LOX }\end{array}$ \\
\hline $3 F G 4$ & 2009 & Neau & $\begin{array}{l}\text { Plexaura } \\
\text { homomalla } \\
8 R \text {-LOX }\end{array}$ & 2.31 & $\begin{array}{l}\text { Crystal structure of Delta413-417:GS } \\
\text { I805A LOX }\end{array}$ \\
\hline 3VF1 & 2012 & Eek & $\begin{array}{l}\text { Germesia } \\
\text { fruticose } \\
11 R \text {-LOX }\end{array}$ & 2.47 & $\begin{array}{l}\text { Structure of a calcium-dependent 11R- } \\
\text { lipoxygenase suggests a mechanism } \\
\text { for Ca-regulation }\end{array}$ \\
\hline
\end{tabular}


Supplemental Table 4. List of all mammalian LOX structures deposited in the PDB. Structures of rabbit 15-LOX, porcine 12-LOX, human 12-LOX, human 15-LOX2 and human 5-LOX are available (five different enzymes).

\begin{tabular}{|c|c|c|c|c|c|}
\hline Code & $\begin{array}{l}\text { Year of } \\
\text { release }\end{array}$ & First author & Organism/Enzyme & $\begin{array}{l}\text { Resolution } \\
(\AA)\end{array}$ & Title \\
\hline $2 \mathrm{POM}$ & 2007 & Choi & Rabbit 15S-LOX & 2.4 & $\begin{array}{l}\text { Revised structure of rabbit } \\
\text { reticulocyte 15S-lipoxygenase }\end{array}$ \\
\hline 1LOX & 1998 & Gillmor & Rabbit 15S-LOX & 2.4 & $\begin{array}{l}\text { RABBIT RETICULOCYTE 15- } \\
\text { LIPOXYGENASE }\end{array}$ \\
\hline 3RDE & 2012 & Funk & Porcine 12-LOX & 1.89 & $\begin{array}{l}\text { Crystal structure of the catalytic } \\
\text { domain of porcine leukocyte 12- } \\
\text { lipoxygenase }\end{array}$ \\
\hline 3D3L & 2008 & Tresaugues & Human 12S-LOX & 2.6 & $\begin{array}{l}\text { The } 2.6 \text { A crystal structure of the } \\
\text { lipoxygenase domain of human } \\
\text { arachidonate } 12 \text {-lipoxygenase, } 12 \mathrm{~S} \text { - } \\
\text { type (CASP Target) }\end{array}$ \\
\hline 4NRE & 2014 & Kobe & Human 15-LOX2 & 2.63 & $\begin{array}{l}\text { The structure of human } 15- \\
\text { lipoxygenase- } 2 \text { with a substrate mimic }\end{array}$ \\
\hline $3 \vee 98$ & 2012 & Gilbert & Human 5-LOX & 2.07 & S663D Stable-5-LOX \\
\hline $3 \vee 92$ & 2012 & Gilbert & Human 5-LOX & 2.74 & S663A Stable-5-LOX \\
\hline $3 \vee 99$ & 2012 & Gilbert & Human 5-LOX & 2.25 & $\begin{array}{l}\text { S663D Stable-5-LOX in complex with } \\
\text { Arachidonic Acid }\end{array}$ \\
\hline $308 Y$ & 2011 & Newcomer & Human 5-LOX & 2.39 & Stable-5-Lipoxygenase \\
\hline
\end{tabular}


Supplemental Table 5. Data collection and refinement statistics for Mn-CspLOX2, the Leu304Val and the Leu304Phe variant.

\begin{tabular}{|c|c|c|c|}
\hline & Mn-CspLOX2 & Leu304Val & Leu304Phe \\
\hline Synchrotron beamline & DESY (Hamburg) & ESRF (Grenoble) & ESRF (Grenoble) \\
\hline Resolution range $(\AA)$ & $50.00-2.00$ & $50.00-2.60$ & $50.00-2.04$ \\
\hline & $(2.10-2.00)$ & $(2.70-2.60)$ & $(2.14-2.04)$ \\
\hline Space group & $P 212121$ & $P 212121$ & $P 212121$ \\
\hline \multirow[t]{2}{*}{ Unit cell $\left(\AA \AA^{\circ}{ }^{\circ}\right)$} & 54.42165 .59167 .35 & 54.42165 .59167 .35 & 54.42165 .59167 .35 \\
\hline & 90.0090 .0090 .00 & 90.0090 .0090 .00 & 90.0090 .0090 .00 \\
\hline Total number of reflections & 446260 (58819) & 243217 (26059) & 495166 (67823) \\
\hline Unique reflections & $102444(13765)$ & 47945 (5028) & $98279(12956)$ \\
\hline Multiplicity & $4.3(4.3)$ & $5.1(5.2)$ & $5.0(5.2)$ \\
\hline Completeness (\%) & $99.3(99.2)$ & $99.6(99.5)$ & $99.8(99.9)$ \\
\hline Mean I/sigma(I) & $14.96(1.74)$ & $18.52(2.76)$ & $19.14(2.80)$ \\
\hline Wilson B-factor $\left(\AA^{2}\right)$ & 39.89 & 67.00 & 44.67 \\
\hline R-merge (\%) & $7.0(79.1)$ & $5.5(67.2)$ & $4.7(56.5)$ \\
\hline $\mathrm{CC} 1 / 2$ & $99.9(72.1)$ & $99.9(83.0)$ & $99.9(82.0)$ \\
\hline Resolution range & $\begin{array}{l}46.28-2.00 \\
(2.02-2.00)\end{array}$ & $\begin{array}{l}46.35-2.60 \\
(2.66-2.60)\end{array}$ & $\begin{array}{l}46.34-2.04 \\
(2.06-2.03)\end{array}$ \\
\hline R-work & 0.1941 (0.2789) & $0.2157(0.3153)$ & $0.1873(0.2606)$ \\
\hline R-free & $0.2239(0.3564)$ & $0.2546(0.3694)$ & $0.2163(0.2826)$ \\
\hline Number of non-hydrogen atoms & 10210 & 9176 & 9919 \\
\hline macromolecules & 9120 & 9118 & 9126 \\
\hline ligands & 2 & 2 & 2 \\
\hline water & 1090 & 58 & 793 \\
\hline Protein residues & 1138 & 1138 & 1138 \\
\hline RMS (bonds) & 0.006 & 0.006 & 0.009 \\
\hline RMS (angles) & 1.088 & 1.301 & 1.292 \\
\hline Ramachandran favored (\%) & 94.89 & 93.75 & 95.6 \\
\hline Ramachandran outliers (\%) & 1.41 & 1.41 & 1.06 \\
\hline Clashscore & 6.14 & 6.08 & 6.08 \\
\hline Average B-factor & 39.6 & 71.4 & 49.0 \\
\hline
\end{tabular}

Statistics for the highest-resolution shell are shown in parentheses. 
Supplemental Table 6. Primers used in this study. Forward primers are indicated with ' $f w d$ ', the reverse primers with ' $r$ ' ${ }^{\prime}$ '. The sequence of the primers is always shown in $5^{\prime}-3$ ' direction.

\begin{tabular}{|c|c|c|}
\hline Construct & Primer & Primer sequence \\
\hline \multirow{2}{*}{$\begin{array}{l}\text { CspLOX2 } \\
\text { Arg57Phe }\end{array}$} & Arg57Phe_fwd & GAAATACTTGATTGAATTCGATAAGGGATTAGTGTCCCTAATTG \\
\hline & Arg57Phe rev & САСТАATCCСТTATCGAATTCAATCAAGTATTTCAATGAAAAC \\
\hline \multirow{2}{*}{$\begin{array}{l}\text { CspLOX2 } \\
\text { Gly60Leu }\end{array}$} & Gly60Leu_fwd & GATTGAGCGAGATAAGCTTTTAGTGTCCCTAATTGCCAATACC \\
\hline & Gly60Leu_rev & CAATTAGGGACACTAAAAGCTTATCTCGCTCAATCAAGTATTTC \\
\hline \multirow{2}{*}{$\begin{array}{l}\text { CspLOX2 } \\
\text { Leu258Val }\end{array}$} & Leu258Val_fwd & GATGGGTTCGTGCTACGTGGCTAATTGCTTCGTGACAATTG \\
\hline & Leu258Val_rev & CGAAGCAATTAGCCACGTAGCACGAACCCATCTGATCTTAG \\
\hline \multirow{2}{*}{$\begin{array}{l}\text { CspLOX2 } \\
\text { Leu263Trp }\end{array}$} & Leu263Trp_fwd & CTTAGCACGAACCCATTGGATCTTAGAACCTTTTGTGCTGGC \\
\hline & Leu263Trp_rev & CAAAAGGTTCTAAGATCCAATGGGTTCGTGCTAAGTGGCTAATTG \\
\hline \multirow{2}{*}{$\begin{array}{l}\text { CspLOX2 } \\
\text { Ile296Ala }\end{array}$} & Ile296Ala_fwd & CCAATTTACCTTGGCCGCTAATGAACTGGCACGAGAACAG \\
\hline & Ile296Ala_rev & GTGCCAGTTCATTAGCGGCCAAGGTAAATTGGAAATGGGG \\
\hline \multirow{2}{*}{$\begin{array}{l}\text { CspLOX2 } \\
\text { Ala300Gly }\end{array}$} & Ala300Gly_fwd & CCTTGGCCATTAATGAGCTCGGACGAGAACAGTTGATCAGTGC \\
\hline & Ala300Gly_rev & CAACTGTTCTCGTCCGAGCTCATTAATGGCCAAGGTAAATTGG \\
\hline \multirow{2}{*}{$\begin{array}{l}\text { CspLOX2 } \\
\text { Leu304Ala }\end{array}$} & Leu304Ala_fwd & CTGGCACGAGAACAGGCGATCAGTGCCGGAGGTTATGCCGATG \\
\hline & Leu304Ala_rev & CCTCCGGCACTGATCGCCTGTTCTCGTGCCAGTTCATTAATG \\
\hline \multirow{2}{*}{$\begin{array}{l}\text { CspLOX2 } \\
\text { Leu304Phe }\end{array}$} & Leu304Phe_fwd & CTGGCACGAGAACAGTTTATCAGTGCCGGAGGTTATGCCGATG \\
\hline & Leu304Phe_rev & CCTCCGGCACTGATAAACTGTTCTCGTGCCAGTTCATTAATG \\
\hline \multirow{2}{*}{$\begin{array}{l}\text { CspLOX2 } \\
\text { Leu304Val }\end{array}$} & Leu304Val_fwd & CTGGCACGAGAACAGGTGATCAGTGCCGGAGGTTATGCCGATG \\
\hline & Leu304Val_rev & CCTCCGGCACTGATCACCTGTTCTCGTGCCAGTTCATTAATG \\
\hline \multirow{2}{*}{$\begin{array}{l}\text { CspLOX2 } \\
\text { Ile305Phe }\end{array}$} & Ile305Phe_fwd & GAACTGGCACGAGAACAGCTGTTCAGTGCCGGAGGTTATGCCG \\
\hline & Ile305Phe_rev & CCTCCGGCACTGAACAGCTGTTCTCGTGCCAGTTCATTAATGG \\
\hline \multirow{2}{*}{$\begin{array}{l}\text { CspLOX2 } \\
\text { Tyr310lle }\end{array}$} & Tyr310lle_fwd & GATCAGTGCCGGAGGTATTGCCGATGATCTGCTCGCTGGAAC \\
\hline & Tyr310lle_rev & GAGCAGATCATCGGCAATACCTCCGGCACTGATCAACTGTTC \\
\hline \multirow{2}{*}{$\begin{array}{l}\text { CspLOX2 } \\
\text { Tyr310Phe }\end{array}$} & Tyr310Phe_fwd & GATCAGTGCCGGAGGTTTTGCCGATGATCTGCTCGCTGGAAC \\
\hline & Tyr310Phe_rev & GAGCAGATCATCGGCAAAACCTCCGGCACTGATCAACTGTTC \\
\hline \multirow{2}{*}{$\begin{array}{l}\text { CspLOX2 } \\
\text { Ala311Phe }\end{array}$} & Ala311Phe_fwd & CAGTGCCGGAGGTTATTTCGATGATCTGCTCGCTGGAACCC \\
\hline & Ala311Phe_rev & GCGAGCAGATCATCGAAATAACCTCCGGCACTGATCAACTG \\
\hline \multirow{2}{*}{$\begin{array}{l}\text { CspLOX2 } \\
\text { Leu315Met }\end{array}$} & Leu315Met_fwd & GCCGATGATCTGATGGCCGGCACCCTTGAAGCCTCTATCGCTG \\
\hline & Leu315Met_fwd & GAGGCTTCAAGGGTGCCGGCCATCAGATCATCGGCATAACCTC \\
\hline \multirow{2}{*}{$\begin{array}{l}\text { CspLOX2 } \\
\text { Leu315Phe }\end{array}$} & Leu315Phe_fwd & GCCGATGATCTGTTCGCCGGCACCCTTGAAGCCTCTATCGCTG \\
\hline & Leu315Phe_rev & GAGGCTTCAAGGGTGCCGGCGAACAGATCATCGGCATAACCTC \\
\hline \multirow{2}{*}{$\begin{array}{l}\text { CspLOX2 } \\
\text { Leu315Val }\end{array}$} & Leu315Val_fwd & GCCGATGATCTGGTCGCCGGCACCCTTGAAGCCTCTATCGCTG \\
\hline & Leu315Val_rev & GAGGCTTCAAGGGTGCCGGCGACCAGATCATCGGCATAACCTCC \\
\hline \multirow{2}{*}{$\begin{array}{l}\text { CspLOX2 } \\
\text { Leu502Val }\end{array}$} & Leu502Val_fwd & GCAAGGCAACTGGAGGTGATGAGAACGCTGTGTGTTTTC \\
\hline & Leu502Val_rev & CACAGCGTTCTCATCACCTCCAGTTGCCTTGCTGCCAACTT \\
\hline \multirow{2}{*}{$\begin{array}{l}\text { CspLOX2 } \\
\text { Leu506Val }\end{array}$} & Leu506Val_fwd & GGAGTTGATGAGAACGGTGTGTGTTTTCTATCCCAATCG \\
\hline & Leu506Val_rev & GATAGAAAACACACACCGTTCTCATCAACTCCAGTTGCC \\
\hline \multirow{2}{*}{$\begin{array}{l}\text { CspLOX1 } \\
\text { Gly401Ala }\end{array}$} & Gly401Ala_fwd & ATATTAATCGCCGTGCAGATGATTTACTGGTAGAACCG \\
\hline & Gly401Ala_rev & CCAGTAAATCATCTGCACGGCGATTAATATTAATAATG \\
\hline \multirow{2}{*}{$\begin{array}{l}\text { CspLOX1 } \\
\text { Tyr310Leu }\end{array}$} & Tyr310Leu_fwd & GAAGCGATCGAGCATCTAGCTAAGGCTCATGTGCAGATGG \\
\hline & Tyr310Leu_rev & CACATGAGCCTTAGCTAGATGCTCGATCGCTTCTCCGTCAAA \\
\hline \multirow{2}{*}{$\begin{array}{l}\text { CspLOX1 } \\
\text { Phe413Ala }\end{array}$} & Phe413Ala_fwd & CCGAACCTTGGCTTAGCTGTGACTAACGGTCCGTTAAC \\
\hline & Phe413Ala_rev & ACCGTTAGTCACAGCTAAGCCAAGGTTCGGTTCTACCAG \\
\hline \multirow{2}{*}{$\begin{array}{l}\text { CspLOX1 } \\
\text { Ile617Leu }\end{array}$} & Ile617Leu_fwd & CCAATCAGGTTTATCTCTTCAGTGTCTTAAATGGC \\
\hline & Ile617Leu_rev & AGACACTGAAGAGATAAACCTGATTGGTTGCTTCTATG \\
\hline \multirow{3}{*}{$\begin{array}{l}\text { CspLOX1 } \\
\text { truncated } \\
44-668 / 180-668\end{array}$} & CspLOX1_Ile668_rev & CAAGCTTCTAAATATTAATTAAAGTGCG \\
\hline & CspLOX1_Glu180_fwd & CCGGATCCGAAGAAACCCCCGATGATTAC \\
\hline & CspLOX1_Ala44_fwd & CCGGATCCGCTATCACCAAACCCATCG \\
\hline
\end{tabular}




\section{Affidavit}

I hereby declare that I wrote this dissertation on my own and with no other sources and aids than quoted.

Julia Newie

Göttingen, January 2016 


\section{Danke!}

Danken möchte ich in erster Linie meinem Doktorvater Prof. Dr. Ivo Feußner für die Unterstützung während der letzten Jahre, für all die Möglichkeiten, die mir im Rahmen der Arbeit geboten wurden und die verschiedenen Herausforderungen, an denen ich gewachsen bin.

Danken möchte ich auch Prof. Dr. Kai Tittmann für das Lesen und Bewerten dieser Arbeit, aber auch für die eine oder andere Geschichte von spannenden Enzymen, für hilfreiche Ratschläge und den nötigen „Blick über den Tellerrand“.

Außerdem danke ich Prof. Dr. Ralf Ficner, Prof. Dr. Marina Bennati und Prof. Dr. Ricardo Mata für die spannenden Kooperationen, die während meiner Promotion zustande gekommen sind und die entscheidende Ergebnisse zu dieser Dissertation beigetragen haben. Ich möchte mich bei den beteiligten Arbeitsgruppen sowie Dr. Alexandra Andreou auch für die produktive Zusammenarbeit bei den Veröffentlichungen bedanken.

Besonders danken möchte ich auch Dr. Piotr Neumann für die kompetente Hilfe bei vielfältigen kristallographischen Problemen und dafür, dass Deine Tür immer für mich offen stand. Es war mir eine große Freude mit Dir zusammenzuarbeiten!

Bei Dr. Müge Kasanmascheff möchte ich mich für die EPR Messungen bedanken. Vielen Dank für die interessante Kooperation und Deine freundliche Art.

Ebenso möchte ich Martin Werner für die Simulationen und Berechnungen danken, aber auch für das Korrekturlesen entsprechender Abschnitte und die Geduld beim Beantworten meiner Fragen.

Für die ACP-AES Messungen möchte ich mich bei Uta Nüsse-Hahne und Dr. Dietrich Hertel bedanken sowie für die UPLC-MS Messungen bei Dr. Kirstin Feußner.

I further want to thank Prof. Dr. Marcia Newcomer for the friendly supervision during my stay in Louisiana. I learned so much! Thanks for welcoming me so warmly and thanks for the great experience.

Dr. Florian Brodhun danke ich für seine nützlichen Ratschläge im Laufe dieser Arbeit sowie für das Korrekturlesen. Es war sehr hilfreich, einen kompetenten Ansprechpartner wie Dich für proteinbiochemische Fragen in der Nähe zu haben!

Dem IRTG 1422 danke ich für die finanzielle Unterstützung bei Forschungsreisen, Sommerschulen und mittels des Stipendiums, aber auch für die interessanten Workshops und Seminare. Ich konnte mein chemisches Wissen vertiefen und habe von der interdisziplinären Arbeit sehr profitiert.

Außerdem danke ich dem Fonds der Chemischen Industrie für die Finanzierung über das Doktorandenstipendium und für die Workshops, auf denen ich andere junge Wissenschaftler kennenlernen konnte.

Herzlich bedanken möchte ich mich an dieser Stelle auch bei meinen lieben Laborkollegen für die hilfsbereite und freundliche Atmosphäre von der man gern Teil ist. Es war schön, mit Euch Luftpolsterfolie zu knacken, beim Altstadtlauf und Staffeltriathlon anzutreten sowie Mittags- und Kaffeepausen gemeinsam zu verbringen. Meinen „First Floor Hard Workers" danke ich dabei besonders für die angenehme, motivierende und durchaus unterhaltsame Zeit im Labor. Danke Ellen, für Deine Hilfe in allen Bereichen und für das Korrekturlesen, Benji für Deine aufgeschlossene Art und die beeindruckenden Zaubertricks, Dan für Deine „cuteness" und Steffen für den abwechslungsreichen Musikgeschmack. Danken möchte ich auch Sven für die Hilfe beim Drucken und Sabine dafür, dass die Zeit an den HPLCs nie langweilig war und für die schönen Spitznamen.

Schließlich geht mein ganz besonderer Dank an meine Eltern und meine Familie, die immer hinter mir standen und mir stets gezeigt haben, wie stolz sie sind, egal wohin mein Weg mich auch führt.

Zuletzt, ganz vertraut, danke ich Jonas, der mir immer wieder zeigt, welch schöne Dinge es abseits der Biochemie noch zu entdecken gibt. Du hast mir mit deiner Zuversicht die nötige Ruhe gegeben, mit der sich so eine Doktorarbeit wesentlich leichter schreibt. 